\title{
Multivalent and dynamic interactions of the influenza virus at lipid membrane interfaces
}

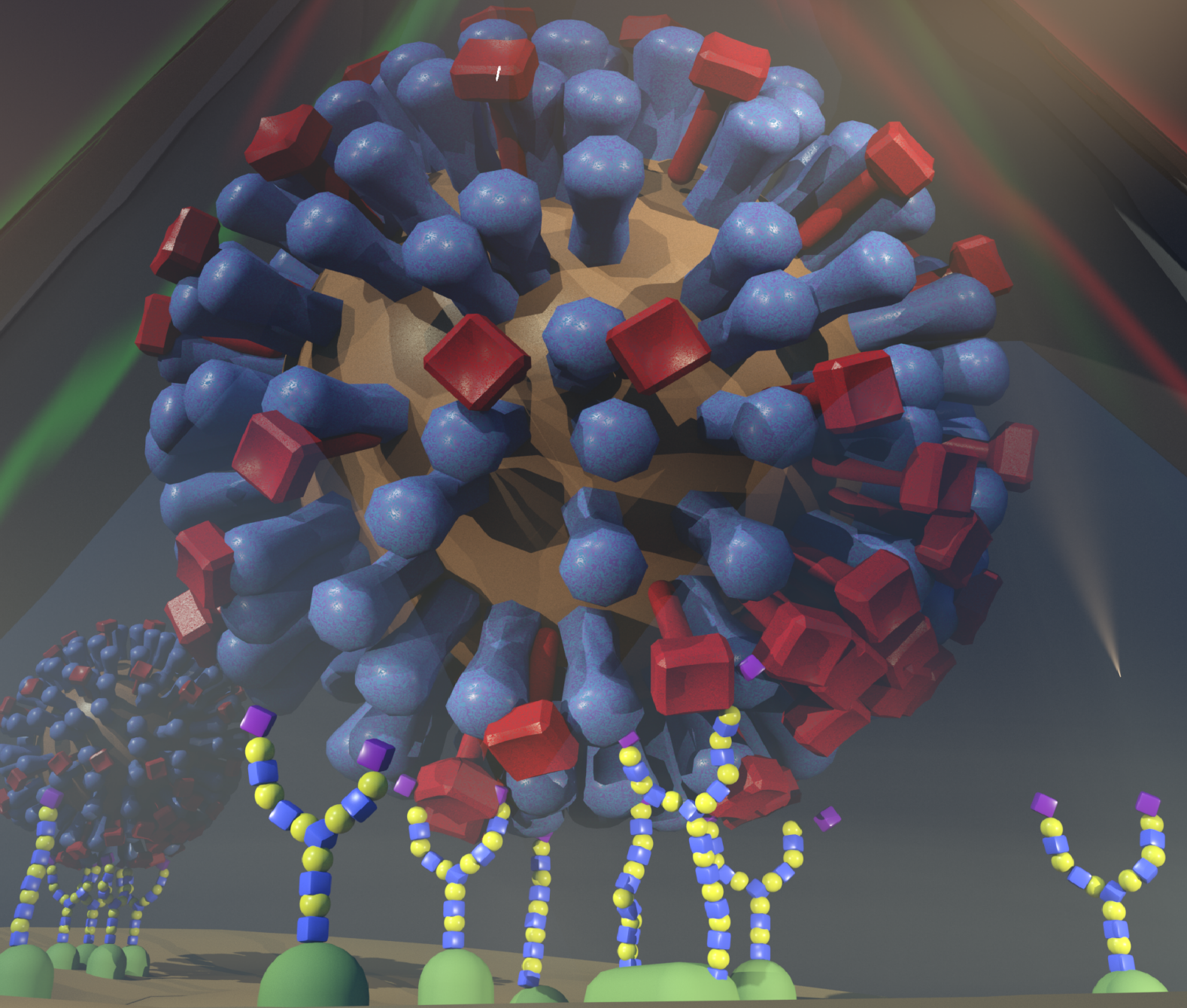

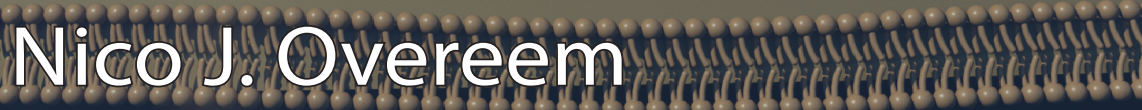




\section{MULTIVALENT AND DYNAMIC INTERACTIONS OF THE INFLUENZA VIRUS AT LIPID MEMBRANE INTERFACES}

Nico Johan Overeem 
Dit proefschrift is goedgekeurd door:

\section{Promotor}

prof. dr. ir. J. Huskens

\section{Co-promotor}

dr. E. van der Vries 


\title{
MULTIVALENT AND DYNAMIC INTERACTIONS OF THE INFLUENZA VIRUS AT LIPID MEMBRANE INTERFACES
}

\author{
PROEFSCHRIFT
}

\author{
ter verkrijging van \\ de graad van doctor aan de Universiteit Twente, \\ op gezag van de rector magnificus, \\ prof. dr. ir. A. Veldkamp, \\ volgens besluit van het College voor Promoties \\ in het openbaar te verdedigen \\ op vrijdag 26 februari 2021 om 14.45 uur
}

door

\section{Nico Johan Overeem}

geboren op 2 maart 1993

te Deventer 
Printed by:

ISBN:

DOI:
Ridderprint

978-90-365-5116-8

$10.3990 / 1.9789036551168$

The research described in this thesis was performed within the laboratories of the Molecular NanoFabrication (MnF) group, at the MESA+ Institute for Nanotechnology, and the Faculty of Science and Technology (TNW) of the University of Twente. This research was supported by the Volkswagen Foundation (FlapChips project) and the Netherlands Organization for Scientific Research (NWO, TOP 715.015.001).

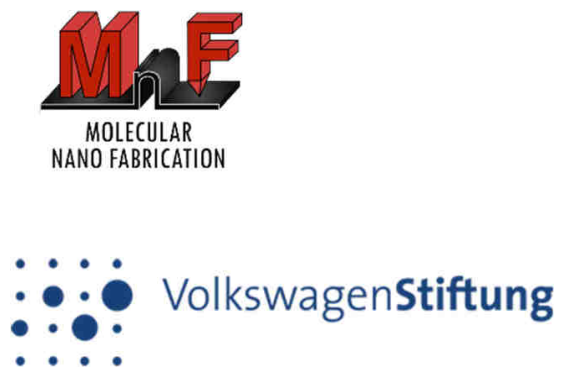

\section{\begin{tabular}{l|l} 
UNIVERSITY & MESA+ \\
OF TWENTE. & INSTITUTE
\end{tabular}}

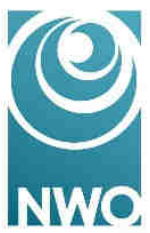

(C) 2020 Nico Johan Overeem, The Netherlands. All rights reserved. No parts of this thesis may be reproduced, stored in a retrieval system, or transmitted in any form or by any means without permission of the author. Alle rechten voorbehouden. Niets uit deze uitgave mag worden vermenigvuldigd, in enige vorm of op enige wijze, zonder voorafgaande schriftelijke toestemming van de auteur. 


\section{PROMOTIECOMMISSIE:}

$\begin{array}{lll}\text { Voorzitter } & \text { prof. dr. J.L. Herek } & \text { (Universiteit Twente) } \\ \text { Promotor: } & \text { prof. dr. ir. J. Huskens } & \text { (Universiteit Twente) } \\ \text { Co-promotor: } & \text { dr. E. van der Vries } & \begin{array}{l}\text { (Royal GD) } \\ \text { (Universitair Medisch Centrum Utrecht) }\end{array} \\ \text { Leden: } & \begin{array}{l}\text { prof. dr. R. Haag } \\ \text { prof. dr. ir. M.W.J. Prins } \\ \text { prof. dr. A. Kocer } \\ \text { prof. dr. ir. P. Jonkheijm }\end{array} & \begin{array}{l}\text { (Freie Universität Berlin) } \\ \text { (Techische Universiteit Eindhoven) }\end{array} \\ & \text { (Universiteit Twente) }\end{array}$





\section{Glossary}

\section{List of symbols}

\begin{tabular}{|c|c|}
\hline$a$ & Particle diameter (m) \\
\hline$A_{\text {contact }}$ & $\begin{array}{l}\text { Contact area in which receptors can be reached by HA on a surface- } \\
\text { bound virus }\left(\mathrm{cm}^{2}\right)\end{array}$ \\
\hline$A_{\text {ex }}$ & Area excluded by a bound virus particle $\left(\mathrm{dm}^{2}\right.$ or $\left.\mathrm{nm}^{2}\right)$ \\
\hline$\alpha$ & Superselectivity parameter (-) \\
\hline$c_{\mathrm{R}}{ }^{\circ}$ & Intrinsic transition point (-) \\
\hline$C^{\circ}$ R,eff & Intrinsic transition point under an applied force (-) \\
\hline$d$ & Diameter (m or $n m)$ \\
\hline$D$ & Diffusion coefficient $\left(\mathrm{cm}^{2} \mathrm{~s}^{-1}\right)$ \\
\hline$\delta Z$ & Distance of a particle from the wall ( $\mathrm{m}$ or $\mathrm{nm}$ ) \\
\hline$\Delta G_{\text {lig,cnf }}$ & Additional free energy cost for making a ligand-receptor bond $(\mathrm{J} / \mathrm{mol})$ \\
\hline$\Delta G_{N S}$ & Free energy of nonspecific binding $(\mathrm{J} / \mathrm{mol})$ \\
\hline$E M$ & Effective molarity (M) \\
\hline$f$ & Faxén correction factor (-) \\
\hline$F$ & Hydrodynamic force ( $\mathrm{N}$ or $\mathrm{fN}$ ) \\
\hline$F_{\text {pull }}$ & Pulling force ( $\mathrm{N}$ or $\mathrm{fN}$ ) \\
\hline$h$ & Height of a microchannel (m) \\
\hline $\bar{I}$ & Average fluorescence intensity (AU) \\
\hline $\bar{I}_{\mathrm{bg}}$ & Average fluorescence intensity of the background $(A U)$ \\
\hline$I_{\mathrm{i}}$ & Local fluorescence intensity (AU) \\
\hline $\mathrm{IC}_{50}$ & Half maximal inhibitory concentration ( $\mathrm{M}$ or $\mu \mathrm{M}$ ) \\
\hline$k$ & Spring constant $\left(\mathrm{N} / \mathrm{m}\right.$ or $\left.\mathrm{J} \cdot \mathrm{mol}^{-1} \cdot \mathrm{nm}^{-2}\right)$ \\
\hline$k_{\mathrm{B}}$ & Boltzmann's constant $\left(1.38 \cdot 10^{-23} \mathrm{~J} / \mathrm{K}\right)$ \\
\hline$k_{\text {cat }}$ & Enzymatic rate constant $\left(\mathrm{s}^{-1}\right)$ \\
\hline$k_{\text {off }}$ & Dissociation rate constant $\left(\mathrm{s}^{-1}\right)$ \\
\hline$k_{\text {on }}$ & Association rate constant $\left(\mathrm{s}^{-1}\right)$ \\
\hline$K_{\mathrm{av}}$ & $\begin{array}{l}\text { Avidity constant, equilibrium constant of the association of a } \\
\text { multivalent interaction }\left(\mathrm{M}^{-1}\right)\end{array}$ \\
\hline
\end{tabular}


$K_{\mathrm{D}}$ Dissociation constant, equilibrium constant of a dissociating complex, inverse of $K_{\mathrm{i}}$ or $K_{\mathrm{av}}(\mathrm{M})$

$K_{\mathrm{i}} \quad$ Individual binding constant, equilibrium constant of the association of a single receptor-ligand interaction $\left(\mathrm{M}^{-1}\right)$

$l \quad$ Length $(\mathrm{m})$

$L \quad$ Length of the legs of a molecular spider ( $m$ or $\mathrm{nm}$ )

$\lambda_{\mathrm{em}} \quad$ Emission wavelength $(\mathrm{nm})$

$\lambda_{\mathrm{ex}} \quad$ Excitation wavelength $(\mathrm{nm})$

$\mu \quad$ Viscosity $\left(\mathrm{kg} \cdot \mathrm{m}^{-1} \cdot \mathrm{s}^{-1}\right)$

$\tilde{\mathrm{N}}$

Average number of possible simultaneous interactions between a virus and receptors on a surface (-)

$N_{\mathrm{A}}$

Avogadro's number $\left(6.022 \cdot 10^{23} \mathrm{~mol}^{-1}\right)$

$N_{\mathrm{L}}$

Number of participating ligands (-)

$\bar{N}_{\mathrm{NA}}$

Average number of NA-receptor interactions per virus (-)

$r$

Radius of a particle ( $\mathrm{m}$ or $\mathrm{nm}$ )

$R$

Ideal gas constant $\left(8.314 \mathrm{~J} \cdot \mathrm{mol}^{-1} \cdot \mathrm{K}^{-1}\right)$

Re Reynolds number (-)

$R_{\mathrm{p}}$

Particle Reynolds number (-)

$\rho$

Density $\left(\mathrm{kg} / \mathrm{m}^{3}\right)$

$\bar{\rho}_{\mathrm{R}}$

Average receptor density $\left(\mathrm{pmol} / \mathrm{cm}^{2}\right)$

$\rho_{\mathrm{R}, \mathrm{I}}$

Local receptor density $\left(\mathrm{pmol} / \mathrm{cm}^{2}\right)$

$S$

Selectivity towards high receptor density over low receptor density (-)

$\sigma_{\mathrm{HA}}$

Density of HA receptor binding domains on a virus $\left(\mathrm{pmol} / \mathrm{cm}^{2}\right)$

$\sigma_{\mathrm{L}}$

Density of ligands or receptor binding domains on a virus $\left(\mathrm{pmol} / \mathrm{cm}^{2}\right)$

$\sigma_{\mathrm{R}}$

Density of receptors on a surface $\left(\mathrm{pmol} / \mathrm{cm}^{2}\right)$

$t^{2} / 2$

Time until half of the saturation coverage is reached (s or min)

$T$

Temperature (K)

$T_{\mathrm{m}}$

Phase transition temperature of lipids between fluid and gel state $\left({ }^{\circ} \mathrm{C}\right)$

$\theta$

Virus coverage (-)

$U$

Average flow velocity $(\mathrm{m} / \mathrm{s})$

$[V]$

Virus concentration ( $\mathrm{M}$ or $\mathrm{vp} / \mathrm{ml}$ )

$V_{\mathrm{ex}}$

Volume excluded by a bound virus particle $\left(\mathrm{dm}^{3}\right.$ or $\left.\mathrm{nm}^{3}\right)$

Vexplore

Volume accessible to a receptor $\left(\mathrm{dm}^{3}\right.$ or $\left.\mathrm{nm}^{3}\right)$

$\dot{y}$

Shear rate $\left(\mathrm{s}^{-1}\right)$ 


\section{List of abbreviations}

AF488

ATTO 565

BLI

Cav1.2

CF350

DHPE

DOPC

DOPE

$\mathrm{FcMeOH}$

Gal

GIcNAc

HA

IAV

LN

LUV

MAP

MD

MLV

MOPS

MPPC

MST

NA

Neu5Ac

NMR

NP

OC

PBS

PDMS

PR8

QCM
Alexa Fluor 488, a green-fluorescent dye

Name of a red-fluorescent dye

Biolayer interferometry

A voltage dependent calcium channel, receptor glycoprotein for the influenza virus

Name of a blue-fluorescent dye

1,2-dihexadecanoyl-sn-glycero-3-phosphoethanolamine, a lipid

1,2-dioleoyl-sn-glycero-3-phosphocholine, a lipid

1,2-dioleoyl-sn-glycero-3-phosphoethanolamine, a lipid hydroxymethylferrocene

Galactose

$\mathrm{N}$-acetyl glucosamine

Hemagglutinin

Influenza A virus

$\mathrm{N}$-acetyl lactosamine

Large unilamellar vesicle

Multivalent affinity profiling

Molecular dynamics

Multilamellar vesicle

3-(N-morpholino)propanesulfonic acid

1-myristoyl-2-palmitoyl-sn-glycero-3-phosphocholine, a lipid

Microscale thermophoresis

Neuraminidase

$\mathrm{N}$-acetylneuraminic acid, a type of sialic acid

Nuclear magnetic resonance

Nucleoprotein

Oseltamivir carboxylate

Phosphate-buffered saline

Polydimethylsiloxane

Influenza virus A/Puerto Rico/8/34 (H1N1) Mount Sinai strain

Quartz crystal microbalance 


$\begin{array}{ll}\text { QCM-D } & \text { Quartz crystal microbalance with dissipation monitoring } \\ \text { R18 } & \text { Octadecyl rhodamine B, a red-fluorescent membrane dye } \\ \text { RBD } & \text { Receptor binding domain } \\ \text { RTK } & \text { Receptor tyrosine kinase } \\ \text { SAv } & \text { Streptavidin } \\ \text { SDS } & \text { Sodium dodecyl sulfonate } \\ \text { Sia } & \text { Sialic acid } \\ \text { SLB } & \text { Supported lipid bilayer } \\ \text { SLN } & \text { Sialyl N-acetyl lactosamine } \\ \text { SNFG } & \text { Symbol nomenclature for glycans } \\ \text { SPR } & \text { Surface plasmon resonance } \\ \text { TEM } & \text { Transmission electron microscopy } \\ \text { TIRF } & \text { Total internal reflection fluorescence microscopy } \\ \text { vp } & \text { Virus particles } \\ \text { X-31 } & \text { An influenza virus strain with HA and NA from A/Aichi/2/68 (H3N2) and } \\ \text { Zan } & \text { other genes from PR8 } \\ & \text { Zanamivir }\end{array}$




\section{Table of contents}

Glossary I

List of symbols I

List of abbreviations III

Table of contents $\quad \mathrm{V}$

Chapter 1. General introduction 1

1.1 References 5

Chapter 2. A dynamic, supramolecular view on the multivalent influenza virus-host cell interaction 11

2.1 Introduction $\quad 12$

2.2 The influenza virus is a multivalent nanoparticle 12

$\begin{array}{ll}\text { 2.2.1. Superselectivity } & 17\end{array}$

2.2.2 Receptor recruitment 18

$\begin{array}{ll}\text { 2.2.3 Dynamic complexes } & 18\end{array}$

2.3 Techniques to measure the binding of influenza viruses 21

2.3.1 Hemagglutination assay 22

2.3.2 Glycan microarrays $\quad 22$

2.3.3 Surface plasmon resonance 23

2.3.4 Biolayer interferometry 23

2.3.5 QCM-D 23

2.3.6 Multivalent affinity profiling $\quad 24$

2.4 Dynamic interactions during initial stages of infection 25

2.4.1 The influence of NA on binding 25 
2.4.2 Binding and cleaving in the mucus

2.4.3 Dynamic interactions of influenza on host cells

2.4.4 Recognition and endocytosis through multivalent interactions

2.5 Conclusions

2.6 References

Chapter 3. Time-dependent binding of molecules and nanoparticles at receptor-modified supported lipid bilayer gradients in a microfluidic device

3.1 Introduction

3.2 Results and discussion

3.2.1 Gradient formation in SLBs

3.2.2 Time-dependent binding of small molecules 50

3.2.3 Dual inlet flow cell to avoid nonspecific binding 59

3.2.4 Time-dependent binding of nanoparticles 60

3.3 Conclusions 65

3.4 Acknowledgements 66

3.5 Experimental 66

3.5.1 Reagents 66

3.5.2 Chip fabrication 66

3.5.3 PDMS flow channel 67

3.5.4 PDMS bonding $\quad 67$

3.5.5 Vesicle preparation $\quad 67$

3.5.6 SLB formation 68

3.5.7 ATTO 565-biotin binding studies 68

3.5.8 Data analysis 69

3.5.9 Nanoparticle binding studies 69 
3.5.10 Finite element simulations $\quad 70$

$\begin{array}{ll}3.6 \text { References } & 70\end{array}$

Chapter 4. Hierarchical multivalent effects control influenza host specificity

4.1 Introduction 74

4.2 Results and discussion $\quad 75$

4.2.1 Multivalent affinity profiling $\quad 75$

4.2.2 Threshold density dependence on receptor type and length 77

4.2.3 A theoretical model to describe the multivalent binding of influenza virus 80

4.2.4 Molecular modelling of HA-glycan binding 82

4.2.5 Discussion $\quad 85$

$\begin{array}{ll}4.3 \text { Conclusions } & 87\end{array}$

$\begin{array}{ll}4.4 \text { Acknowledgements } & 87\end{array}$

$\begin{array}{ll}4.5 \text { Experimental } & 87\end{array}$

4.5.1 Biotinylated glycan preparation $\quad 87$

4.5.2 Virus stock preparation 88

4.5.3 Virus labelling $\quad 89$

4.5.4 Chip fabrication $\quad 89$

4.5.5 PDMS flow channel 90

4.5.6 PDMS bonding 90

4.5.7 Lipid vesicle preparation 90

4.5.8 SLB formation and functionalization 91

4.5.9 Binding studies 92

4.5.10 Image analysis 92

4.5.11 Binding studies with QCM 92

4.5.12 Binding studies with BLI 93 
4.5.13 Code availability 94

4.5.14 Microarray studies 94

4.5.15 Molecular dynamics 95

4.6 Appendices 98

4.6.1 Derivation and fitting of theoretical binding model 101

4.6.2 Modes of HA-glycan complexation 109

$\begin{array}{ll}4.7 \text { References } & 115\end{array}$

Chapter 5. Direct visualization of the superselective binding of $\begin{array}{ll}\text { influenza viruses } & 120\end{array}$

$\begin{array}{ll}5.1 \text { Introduction } & 122\end{array}$

$\begin{array}{ll}5.2 \text { Results and discussion } & 124\end{array}$

5.2.1 Visualization and quantification of virus binding on receptor $\begin{array}{ll}\text { gradients } & 124\end{array}$

5.2.5 Effects of virus concentration and flow rate on threshold receptor density

$\begin{array}{ll}5.3 \text { Conclusions } & 139\end{array}$

$\begin{array}{ll}5.4 \text { Acknowledgements } & 140\end{array}$

$\begin{array}{ll}5.5 \text { Experimental } & 140\end{array}$

$\begin{array}{ll}\text { 5.5.1 Materials } & 140\end{array}$

$\begin{array}{ll}\text { 5.5.2 Virus stock preparation } & 140\end{array}$

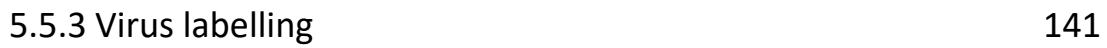

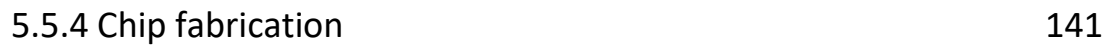

5.5.5 PDMS flow channel 142

5.5.6 PDMS bonding 142

5.5.7 Lipid vesicle preparation 142

5.5.8 SLB formation and functionalization 143

$\begin{array}{ll}\text { 5.5.9 Binding studies } & 143\end{array}$ 
$\begin{array}{ll}\text { 5.5.11 Statistical comparisons } & 144\end{array}$

$\begin{array}{ll}5.6 \text { References } & 144\end{array}$

Chapter 6. The role of neuraminidase in the superselective binding of the influenza virus

150

$\begin{array}{ll}6.1 \text { Introduction } & 152\end{array}$

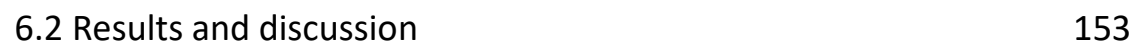

6.2.1 Time-dependent binding studies of IAV with and without NA inhibitor

6.2.2 Towards a theoretical model of superselective virus binding with receptor cleaving

6.2.3 Interactions and structure of single virus particles 165

$\begin{array}{ll}6.3 \text { Conclusions } & 168\end{array}$

$\begin{array}{ll}6.4 \text { Acknowledgements } & 169\end{array}$

$\begin{array}{ll}6.5 \text { Experimental } & 169\end{array}$

$\begin{array}{ll}\text { 6.5.1 Materials } & 169\end{array}$

6.5.2 Virus stock preparation 169

$\begin{array}{ll}\text { 6.5.3 Virus labelling } & 170\end{array}$

$\begin{array}{ll}\text { 6.5.4 Chip fabrication } & 170\end{array}$

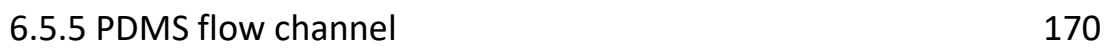

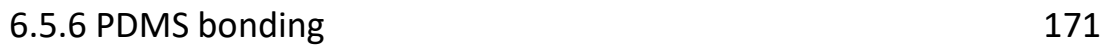

6.5.7 Lipid vesicle preparation 171

6.5.8 SLB formation and functionalization 172

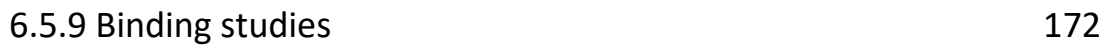

6.5.10 Image analysis 173

6.5.11 Single virus tracking 173

$\begin{array}{ll}\text { 6.5.12 Cryo-TEM } & 174\end{array}$ 
7.2 Molecular spiders and superdiffusive walking

7.3 Motility of influenza virus

7.4 The role of motility in host cell recognition

7.5 Lessons from molecular walkers for influenza

7.6 Conclusions

7.7 Acknowledgements

7.8 References

Chapter 8. Future perspectives

8.1 MAP as a biosensor

204

8.2 Interaction of influenza with the glycocalyx 204

8.3 Antiviral drugs

206

8.4 Application to other pathogens

206

8.4.1 SARS-CoV-2

207

8.5 Cancer therapies

208

8.6 Virus motility

208

8.7 References

208

Summary

Samenvatting

Acknowledgements

229

About the author 


\section{Chapter 1}

\section{General introduction}

Influenza A viruses adapt readily to the immune system of their hosts and to new host species. These adaptations often go together with changes in the interactions of the viruses with their receptors. This chapter introduces influenza A viruses and how their interactions are studied in this thesis.

Influenza is one of the most common diseases in the world. Despite its reputation as a mild disease, it is among the leading causes of death in young people. ${ }^{1}$ This respiratory disease is caused by influenza A and influenza B viruses. ${ }^{2,3}$ In particular the influenza $A$ viruses pose a heavy burden of morbidity and mortality on humanity as well as an economic burden. ${ }^{3,4}$ These viruses may cause outbreaks of zoonotic infections such as with the highly pathogenic avian influenza viruses. ${ }^{5}$ When such zoonotic viruses become transmissible between humans, they can form the origin of a pandemic. ${ }^{6-9}$ Until COVID-19, all recent pandemics of respiratory infections were caused by animal influenza A viruses that adapted to human hosts. ${ }^{10,11}$ Such pandemic viruses spread globally, infecting up to $30 \%$ of the human population and may cause millions of deaths. ${ }^{12}$ The lingering effects of a pandemic are the recurring seasonal epidemics of viruses that evolved from the pandemic virus. ${ }^{2,13}$ These seasonal epidemics affect annually $15 \%$ of the human population, resulting in $3-5$ million hospitalizations and $290000-650000$ deaths per year. ${ }^{14}$

The initiation of an infection by an influenza virus is governed by two glycoproteins, hemagglutinin ( $\mathrm{HA}$ ) and neuraminidase (NA), that make up the characteristic "spikes" on the surface of the virus (Figure 1a). ${ }^{15}$ Different subtypes of HA and NA, denoted H1-16 and N1-9, are distinguished based on antigenic properties and can be exchanged between viral strains by reassortment when different subtypes infect the same host (Figure 1b).,16 All of these subtypes are found in aquatic birds, which act as a reservoir for influenza A viruses (Figure 1c). ${ }^{3}$ Subtypes H17-18 and N10-11 are found exclusively in bats and cannot reassort with other subtypes. ${ }^{17}$ 


\section{Chapter 1}

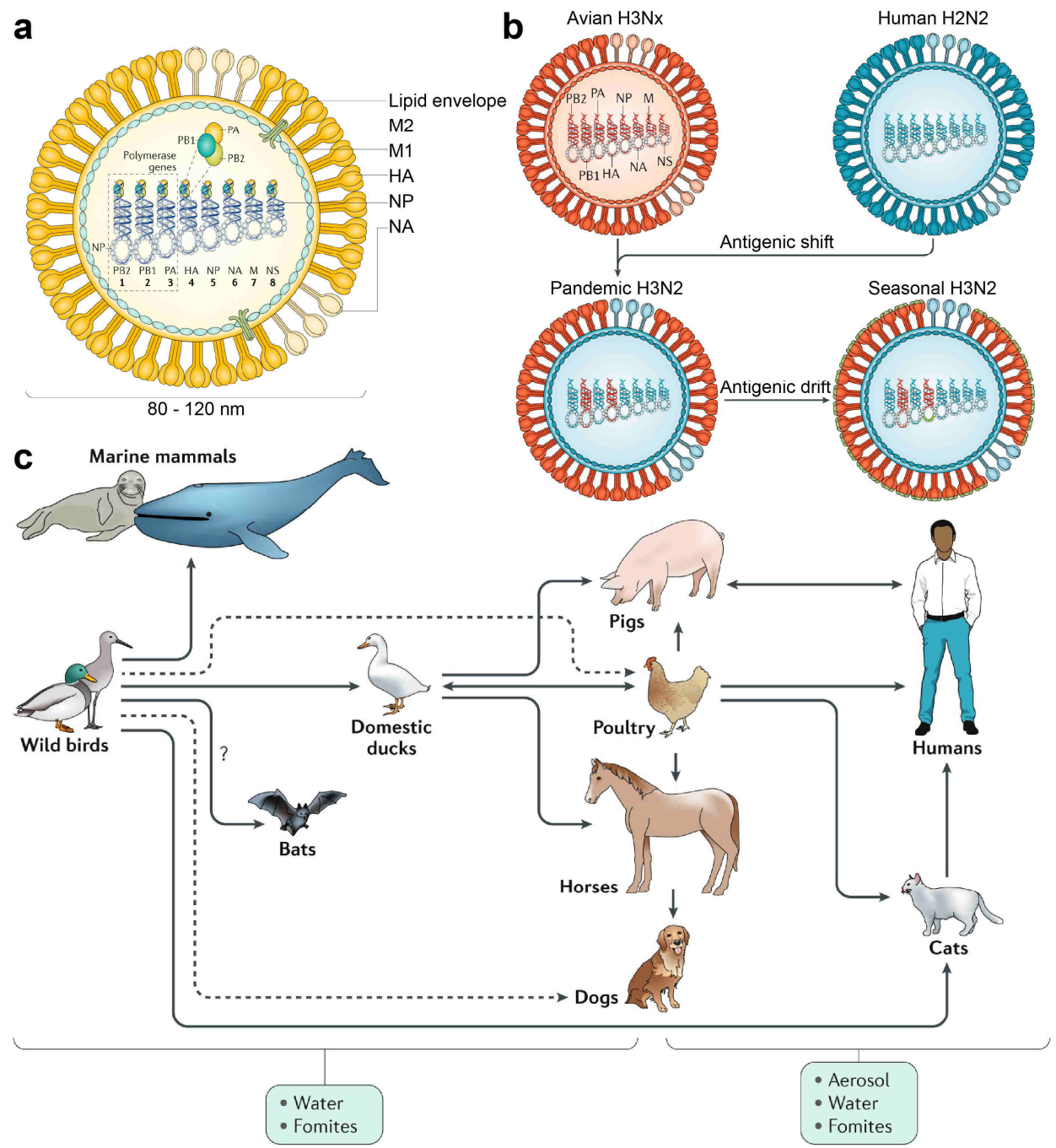

Figure 1| Structure of influenza A virus and its adaptation to host species. a) Influenza A viruses are approximately $100 \mathrm{~nm}$ in diameter and can be spherical or elongated up to several $\mu \mathrm{m}$. The surface of the virus is covered with the proteins hemagglutinin (HA) and neuraminidase (NA). These proteins are presented on a lipid envelope that is supported by matrix protein M2. The membrane also contains an ion channel M1 that plays a role in the assembly and disassembly of the virus. The RNA genome of the virus is packed into eight segments around nucleoprotein (NP). b) Influenza can avoid host immunity by forming reassorted viruses when two different viruses infect the same host 
cell, which is called "antigenic shift". Further adaptation to avoid host immunity by mutations is called "antigenic drift". c) The reservoir for influenza A viruses is wild aquatic birds. From birds, the viruses have adapted to other species directly or via intermediate hosts. Influenza viruses are transmitted via water and fomites. For efficient transmission between terrestrial mammals, influenza viruses have acquired aerosol transmission. Figure reproduced with permission. ${ }^{3}$ Copyright 2018, Springer Nature.

$\mathrm{HA}$ is primarily responsible for the attachment of the virus to its specific host cell receptor. Binding of the virus to the host cell is followed by endocytosis and fusion of the viral and cell membranes. ${ }^{18}$ The receptor specificity of HA is considered a key determinant of pandemic potential in influenza. ${ }^{19,20}$ The HA of avian influenza types binds preferentially to glycans that end in a sialic acid moiety linked by an $\alpha 2,3$-linkage to the penultimate galactose, whereas human influenza HAs favor the $\alpha 2,6$-linkage. ${ }^{16,21}$ The receptor binding domain (RBD) of $\mathrm{HA}$ is also the main target of the adaptive immune response from the host. ${ }^{22,23}$ Although additional mutations are usually required, the evolution of efficient virus transmissibility and escape from host immunity as well as severity of the disease are all associated with amino acid substitutions in the RBD. ${ }^{24-26}$ Mutations in the RBD lead to changes in both the affinity and specificity of HA for different glycan types. ${ }^{27,28}$ The emergence of pandemic strains is associated with a transition from specificity for $\alpha 2,3$ to $\alpha 2,6 .^{8,29}$

The receptor-cleaving enzyme NA binds to the same sialoglycans as HA and hydrolyzes the bond between sialic acid and galactose. ${ }^{20}$ It increases the initial binding rate and avidity but also reverses off-target binding. ${ }^{20,30}$ While the NA of human influenza viruses can also cleave $\alpha 2,6$-linked glycans, it generally maintains a high activity towards the $\alpha 2,3$ linkage that is attributed to the presence of high numbers of $\alpha 2,3$ decoy glycans in the mucus. ${ }^{2,31-33}$ The functional balance of binding and cleaving maintains influenza viruses in a dynamic regime that allows the viruses to transmit and proliferate. ${ }^{7,34-36}$

To recognize potentially pandemic influenza viruses and develop effective antivirals, a thorough understanding of the interactions between the virus and the mucus and glycocalyx of the host is required. ${ }^{37}$ To quantitatively assess these interactions, a method is needed that deconvolutes affinity, specificity, density dependence and structural aspects of the influenza-glycan interactions in a representative environment as well as a physical-mathematical model that 
translates the individual molecular determinants of the affinity and contact area into the overall affinity and specificity. To further understand these interactions, not only at equilibrium but also in their transient states, a timedependent quantification is needed. ${ }^{38}$

This thesis describes the multivalent and dynamic interactions between the influenza virus and sialoglycans and introduces a method to quantitatively assess these interactions using receptor gradients.

Chapter 2 discusses the structure of influenza viruses and their interactions with sialoglycans. The concept of multivalency is explained and how multivalency can lead to superselectivity, recruitment of receptors and the formation of dynamic complexes. Techniques that measure the multivalent binding and receptor specificity of HA are reviewed. The multivalency of influenza virus is proposed to play a key role in traversal of the mucus, host cell recognition, and endocytosis.

Chapter 3 describes the study of the time-dependent binding of analytes onto receptor gradients on supported lipid bilayers (SLBs) in a microfluidic flow cell using fluorescence microscopy and finite-element simulations. This study shows that the loss of analyte outside the sensing area is governed by nonspecific interactions of the lipids, used to form the SLBs, in the tubing and by the diffusion constant of the analyte. Adding a second inlet that decouples the influxes of lipids and analytes is introduced to prevent the analytes from flowing along nonspecifically bound receptors in the tubing and the adsorption profiles in single and double inlet devices are compared.

Chapter $\mathbf{4}$ introduces a method to quantify the avidity of influenza viruses by their threshold receptor density using sialoglycan receptor density gradients on SLBs. This method is used to assess the binding of an influenza virus on gradients with $\alpha 2,3$ and $\alpha 2,6$ glycans of different lengths. Combined with information from multiple platforms, an analytical model, and molecular dynamics simulations, the binding behavior shows how intrinsic structural differences in the binding of $\mathrm{HA}$ to $\alpha 2,3$ and $\alpha 2,6$ receptors are transferred through the length and density of sialoglycans at the cell surface into virus avidity and specificity.

Chapter 5 demonstrates that the superselective binding of influenza viruses can be visualized on receptor density gradients. Quantitative assessment is possible 
if the gradients are prepared around the threshold density and the receptor density on the high side does not lead to crowding. The threshold receptor density is shown to increase under shear flow.

Chapter 6 describes the role of NA in the superselective binding of influenza viruses. The virus binding as function of receptor density is studied over time while the activity of NA is controlled by varying the concentration of NA inhibitor. The contribution of NA to the binding results in a lower threshold receptor density at low concentrations of NA inhibitor but in absence of inhibitor, the receptor-cleaving activity of NA results in a higher apparent threshold receptor density. The receptor-cleaving activity of NA reduces the virus binding at low initial receptor densities more strongly than at high receptor densities, leading to an enhanced selectivity. At the level of individual viruses, a subpopulation of the adsorbed viruses is mobile of which a few show long-range motility. Most of the viruses are spherical but some are elongated.

Chapter 7 reviews the receptor-cleaving action of influenza viruses and compares the virus with artificial receptor-cleaving molecular walkers. Directions are proposed for the development of models that may help to further understand the directional motility of influenza viruses.

\subsection{References}

1. Heron, M. Deaths: leading causes for 2017. National vital statistics reports $68,(2019)$.

2. Neumann, G. \& Kawaoka, Y. Transmission of influenza A viruses. Virology 479-480, 234246 (2015).

3. Krammer, F., Smith, G. J. D., Fouchier, R. A. M., Peiris, M., Kedzierska, K., Doherty, P. C., Palese, P., Shaw, M. L., Treanor, J., Webster, R. G. \& García-Sastre, A. Influenza. Nat. Rev. Dis. Prim. 4, 3 (2018).

4. Klepser, M. E. Socioeconomic impact of seasonal (epidemic) influenza and the role of over-the-counter medicines. Drugs 74, 1467-1479 (2014).

5. Influenza (Avian and other zoonotic). WHO (2018). at <https://www.who.int/newsroom/fact-sheets/detail/influenza-(avian-and-other-zoonotic) >

6. Herfst, S., Schrauwen, E. J. A., Linster, M., Chutinimitkul, S., de Wit, E., Munster, V. J., Sorrell, E. M., Bestebroer, T. M., Burke, D. F., Smith, D. J., Rimmelzwaan, G. F., Osterhaus, A. D. M. E. \& Fouchier, R. A. M. Airborne Transmission of Influenza A/H5N1 Virus Between Ferrets. Science 336, 1534-1541 (2012).

7. $\mathrm{Xu}, \mathrm{R}$., Zhu, X., McBride, R., Nycholat, C. M., Yu, W., Paulson, J. C. \& Wilson, I. A. Functional balance of the hemagglutinin and neuraminidase activities accompanies the emergence of the 2009 H1N1 influenza pandemic. J. Virol. 86, 9221-32 (2012).

8. Imai, M., Watanabe, T., Hatta, M., Das, S. C., Ozawa, M., Shinya, K., Zhong, G., Hanson, 


\section{Chapter 1}

A., Katsura, H., Watanabe, S., Li, C., Kawakami, E., Yamada, S., Kiso, M., Suzuki, Y., Maher, E. a., Neumann, G. \& Kawaoka, Y. Experimental adaptation of an influenza H5 HA confers respiratory droplet transmission to a reassortant $\mathrm{H} 5 \mathrm{HA} / \mathrm{H} 1 \mathrm{~N} 1$ virus in ferrets. Nature 486, 420-428 (2012).

9. Linster, M., Van Boheemen, S., De Graaf, M., Schrauwen, E. J. A., Lexmond, P., Mänz, B., Bestebroer, T. M., Baumann, J., Van Riel, D., Rimmelzwaan, G. F., Osterhaus, A. D. M. E., Matrosovich, M., Fouchier, R. A. M. \& Herfst, S. Identification, characterization, and natural selection of mutations driving airborne transmission of A/H5N1 virus. Cell 157, 329-339 (2014).

10. Schaapveld, M., Aleman, B. M. P., van Eggermond, A. M., Janus, C. P. M., Krol, A. D. G., van der Maazen, R. W. M., Roesink, J., Raemaekers, J. M. M., de Boer, J. P., Zijlstra, J. M., van Imhoff, G. W., Petersen, E. J., Poortmans, P. M. P., Beijert, M., Lybeert, M. L., Mulder, I., Visser, O., Louwman, M. W. J., Krul, I. M., Lugtenburg, P. J. \& van Leeuwen, F. E. Emergence of a Novel Swine-Origin Influenza A (H1N1) Virus in Humans. N. Engl. J. Med. 360, 2605-2615 (2009).

11. Wu, F., Zhao, S., Yu, B., Chen, Y.-M., Wang, W., Song, Z.-G., Hu, Y., Tao, Z.-W., Tian, J.-H., Pei, Y.-Y., Yuan, M.-L., Zhang, Y.-L., Dai, F.-H., Liu, Y., Wang, Q.-M., Zheng, J.-J., Xu, L., Holmes, E. C. \& Zhang, Y.-Z. A new coronavirus associated with human respiratory disease in China. Nature 579, 265-269 (2020).

12. Taubenberger, J. K. \& Morens, D. M. 1918 Influenza: The mother of all pandemics. Emerg. Infect. Dis. 12, 15-22 (2006).

13. Du, X., King, A. A., Woods, R. J. \& Pascual, M. Evolution-informed forecasting of seasonal influenza A (H3N2). Sci. Transl. Med. 9, eaan5325 (2017).

14. Influenza (Seasonal). WHO (2018). at <https://www.who.int/en/news-room/factsheets/detail/influenza-(seasonal)>

15. Stevens, J., Blixt, O., Tumpey, T. M., Taubenberger, J. K., Paulson, J. C. \& Wilson, I. A. Structure and Receptor Specificity of the Hemagglutinin from an H5N1 Influenza Virus. Science 312, 404-410 (2006).

16. Long, J. S., Mistry, B., Haslam, S. M. \& Barclay, W. S. Host and viral determinants of influenza A virus species specificity. Nat. Rev. Microbiol. 17, 67-81 (2019).

17. Ma, W., García-Sastre, A. \& Schwemmle, M. Expected and Unexpected Features of the Newly Discovered Bat Influenza A-like Viruses. PLoS Pathog. 11, 2-7 (2015).

18. Chlanda, P. \& Zimmerberg, J. Protein-lipid interactions critical to replication of the influenza A virus. FEBS Lett. 590, 1940-1954 (2016).

19. Hsu, A. C. Y. Influenza virus: A master tactician in innate immune evasion and novel therapeutic interventions. Front. Immunol. 9, 1-11 (2018).

20. Byrd-Leotis, L., Cummings, R. D. \& Steinhauer, D. A. The Interplay between the Host Receptor and Influenza Virus Hemagglutinin and Neuraminidase. Int. J. Mol. Sci. 18, 1541 (2017).

21. de Graaf, M. \& Fouchier, R. A. M. Role of receptor binding specificity in influenza A virus transmission and pathogenesis. EMBO J. 33, 823-841 (2014).

22. Wu, N. C. \& Wilson, I. A. A Perspective on the Structural and Functional Constraints for Immune Evasion: Insights from Influenza Virus. J. Mol. Biol. 429, 2694-2709 (2017).

23. DeDiego, M. L., Chiem, K. \& Topham, D. J. Directed selection of amino acid changes in 
the influenza hemagglutinin and neuraminidase affecting protein antigenicity. Vaccine 36, 6383-6392 (2018).

24. Koel, B. F., Burke, D. F., Bestebroer, T. M., Vliet, S. van der, Zondag, G. C. M., Vervaet, G., Skepner, E., Lewis, N. S., Spronken, M. I. J., Russell, C. A., Eropkin, M. Y., Hurt, A. C., Barr, I. G., Jong, J. C. de, Rimmelzwaan, G. F., Osterhaus, A. D. M. E., Fouchier, R. A. M. \& Smith, D. J. Substitutions Near the Receptor Binding Site Determine Major Antigenic Change During Influenza Virus Evolution. Science 342, 976-979 (2013).

25. Memoli, M. J., Bristol, T., Proudfoot, K. E., Sally Davis, A., Dunham, E. J. \& Taubenberger, J. K. In vivo evaluation of pathogenicity and transmissibility of influenza A(H1N1)pdm09 hemagglutinin receptor binding domain 222 intrahost variants isolated from a single immunocompromised patient. Virology 428, 21-29 (2012).

26. Maines, T. R., Chen, L. M., Van Hoeven, N., Tumpey, T. M., Blixt, O., Belser, J. A., Gustin, K. M., Pearce, M. B., Pappas, C., Stevens, J., Cox, N. J., Paulson, J. C., Raman, R., Sasisekharan, R., Katz, J. M. \& Donis, R. O. Effect of receptor binding domain mutations on receptor binding and transmissibility of avian influenza H5N1 viruses. Virology 413, 139-147 (2011).

27. Zhang, W., Shi, Y., Lu, X., Shu, Y., Qi, J. \& Gao, G. F. An Airborne Transmissible Avian Influenza H5 Hemagglutinin Seen at the Atomic Level. Science 340, 1463-1468 (2013).

28. Stevens, J., Blixt, O., Glaser, L., Taubenberger, J. K., Palese, P., Paulson, J. C. \& Wilson, I. A. Glycan microarray analysis of the hemagglutinins from modern and pandemic influenza viruses reveals different receptor specificities. J. Mol. Biol. 355, 1143-1155 (2006).

29. Schneider, E., Li, J. \& Velkov, T. A Portrait of the Sialyl Glycan Receptor Specificity of the H10 Influenza Virus Hemagglutinin-A Picture of an Avian Virus on the Verge of Becoming a Pandemic? Vaccines 5, 51 (2017).

30. Guo, H., Rabouw, H., Slomp, A., Dai, M., van der Vegt, F., van Lent, J. W. M., McBride, R., Paulson, J. C., de Groot, R. J., van Kuppeveld, F. J. M., de Vries, E. \& de Haan, C. A. M. Kinetic analysis of the influenza $A$ virus HA/NA balance reveals contribution of NA to virus-receptor binding and NA-dependent rolling on receptor-containing surfaces. PLOS Pathog. 14, e1007233 (2018).

31. Yang, X., Steukers, L., Forier, K., Xiong, R., Braeckmans, K., Van Reeth, K. \& Nauwynck, H. A Beneficiary Role for Neuraminidase in Influenza Virus Penetration through the Respiratory Mucus. PLoS One 9, e110026 (2014).

32. Gaymard, A., Le Briand, N., Frobert, E., Lina, B. \& Escuret, V. Functional balance between neuraminidase and haemagglutinin in influenza viruses. Clin. Microbiol. Infect. 22, 975983 (2016).

33. Baum, L. G. \& Paulson, J. C. The N2 neuraminidase of human influenza virus has acquired a substrate specificity complementary to the hemagglutinin receptor specificity. Virology 180, 10-15 (1991).

34. Yen, H.-L., Liang, C.-H., Wu, C.-Y., Forrest, H. L., Ferguson, A., Choy, K.-T., Jones, J., Wong, D. D.-Y., Cheung, P. P.-H., Hsu, C.-H., Li, O. T., Yuen, K. M., Chan, R. W. Y., Poon, L. L. M. M., Chan, M. C. W. W., Nicholls, J. M., Krauss, S., Wong, C.-H., Guan, Y., Webster, R. G., Webby, R. J. \& Peiris, M. Hemagglutinin-neuraminidase balance confers respiratorydroplet transmissibility of the pandemic H1N1 influenza virus in ferrets. Proc. Natl. Acad. Sci. U.S.A. 108, 14264-14269 (2011). 


\section{Chapter 1}

35. Wagner, R., Matrosovich, M. \& Klenk, H.-D. Functional balance between haemagglutinin and neuraminidase in influenza virus infections. Rev. Med. Virol. 12, 159-166 (2002).

36. Dou, D., Revol, R., Östbye, H., Wang, H. \& Daniels, R. Influenza A Virus Cell Entry, Replication, Virion Assembly and Movement. Front. Immunol. 9, 1-17 (2018).

37. Raman, R., Tharakaraman, K., Shriver, Z., Jayaraman, A., Sasisekharan, V. \& Sasisekharan, R. Glycan receptor specificity as a useful tool for characterization and surveillance of influenza A virus. Trends Microbiol. 22, 632-641 (2014).

38. Benton, D. J., Martin, S. R., Wharton, S. A. \& McCauley, J. W. Biophysical measurement of the balance of influenza A hemagglutinin and neuraminidase activities. J. Biol. Chem. 290, 6516-6521 (2015). 


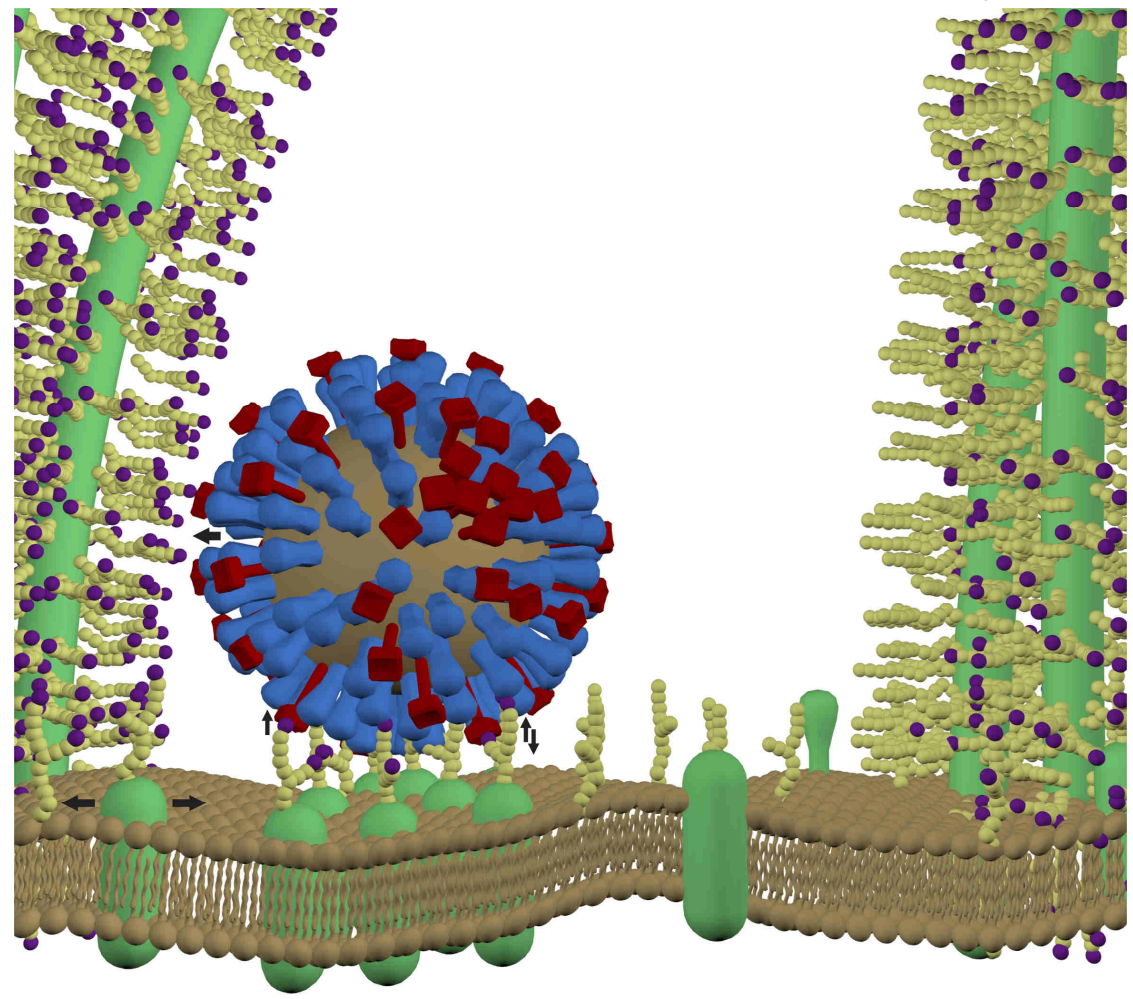

\section{Highlights}

- Influenza A virus displays HA and NA proteins on its surface in a multivalent fashion

- Multivalency allows superselective binding, recruitment of receptors, and formation of dynamic complexes

- Techniques to measure multivalent binding are reviewed

- Receptor-cleaving NA keeps the binding dynamic

- Influenza uses its multivalency to cross the mucus and force endocytosis 


\section{A dynamic, supramolecular view on the multivalent influenza virus- host cell interaction}

The multivalent interactions of influenza viruses are maintained in a dynamic regime by a functional balance between binding and cleaving. Understanding how influenza viruses traverse the mucus and recognize host cells is critical for evaluating their zoonotic potential, and for prevention and treatment of the disease. The surface of the influenza A virus is covered with the receptor-binding protein hemagglutinin (HA) and the receptor-cleaving enzyme neuraminidase (NA), which jointly control the interactions between the virus and the host cell. These proteins are organized in closely spaced trimers and tetramers to facilitate multivalent interactions with sialic acidterminated glycans. This review chapter shows that the individually weak multivalent interactions of influenza viruses allow superselective binding, virus-induced recruitment of receptors, and the formation of dynamic complexes that facilitate molecular walking. Techniques to measure the avidity and receptor specificity of influenza viruses are reviewed, and the pivotal role of multivalent interactions with their emergent properties in crossing the mucus and entering host cells are discussed. A model is proposed for the initiation of cell entry through virus-induced receptor clustering.

Part of this chapter has been published in:

Overeem, N.J., Van der Vries, E., Huskens, J., Small - accepted 


\subsection{Introduction}

Until COVID-19, all recent pandemics of respiratory infections were caused by animal influenza $A$ viruses (IAVs) that crossed the species barrier to humans. The surface of IAV is decorated with the glycoproteins hemagglutinin (HA) and neuraminidase (NA), that are together responsible for the surface interactions of the virus with a host cell. ${ }^{1,2} \mathrm{HA}$ provides a means of host cell recognition to the virus by binding specifically to sialoglycan receptors, while NA prevents aggregation and entrapment of the virus by cleaving off the sialic acid end groups from the same receptors. ${ }^{3}$ The functional balance of receptor binding and cleaving has been recognized as a key factor of virus proliferation and adaptation to different hosts. ${ }^{4,5}$ Only recently, however, the interplay of these two glycoproteins was shown to be responsible for the remarkable efficiency with which IAV crosses the mucus barrier. ${ }^{6}$ We believe that the multivalent character of IAV explains many of the complex interactions between virus and host in the initial stages of infection which remain only superficially understood.

This chapter aims to demonstrate that the behavior of IAVs in the initial stages of infection, starting with the interaction of the viruses at the host cell surface, is intrinsic to their multivalent presentation of weakly binding ligands and receptor-cleaving enzymes. First, we discuss the concept of multivalency with its emergent properties, including how weak multivalency allows interactions that remain dynamic, and then "map" this concept onto the virus-host cell interactions. Secondly, we discuss the available techniques that are used to quantify the multivalent virus-receptor interactions of IAVs. Finally, we discuss how the virus uses multivalent interactions to traverse the mucus, to position itself onto a cell surface, and to manipulate the cell surface forcing endocytosis.

\subsection{The influenza virus is a multivalent nanoparticle}

IAVs are nano-sized particles with diameters of 100-150 nm. ${ }^{7}$ They can be spherical or elongated up to several $\mu \mathrm{m}$. A spherical virion has approximately 290-340 HA trimers and 24-50 NA tetramers. ${ }^{7,8}$ When IAVs bind to a cell, they form multiple interactions with glycoproteins and glycolipids on the cell membrane (Figure 2.1a). ${ }^{9}$ Binding of the virus to the cell surface is facilitated by $\mathrm{HA}$, which binds to sialic acid-terminated glycans with millimolar affinities 
(Figure 2.1b), but controlled by NA, an enzyme that both binds to the same glycan receptors as well as cleaves off the terminal sialic acid from the glycan. ${ }^{3}$ The glycans that act as receptors for IAV are most commonly branched structures that terminate in several $\mathrm{N}$-acetyllactosamine (LN) repeats with sialic acid linked to the penultimate galactose by either an $\alpha 2,3-$ or an $\alpha 2,6$-linkage (Figure 2.1c). ${ }^{10}$ Both types are found in humans, but the HA of human-adapted IAVs binds preferentially to those with the $\alpha 2,6$-linkage (2,6-SLN, found in the upper airways of humans), whereas that of avian IAVs favors the $\alpha 2,3$-linkage (2,3-SLN, found in the lower airways of humans and the intestines of birds). ${ }^{2,11}$

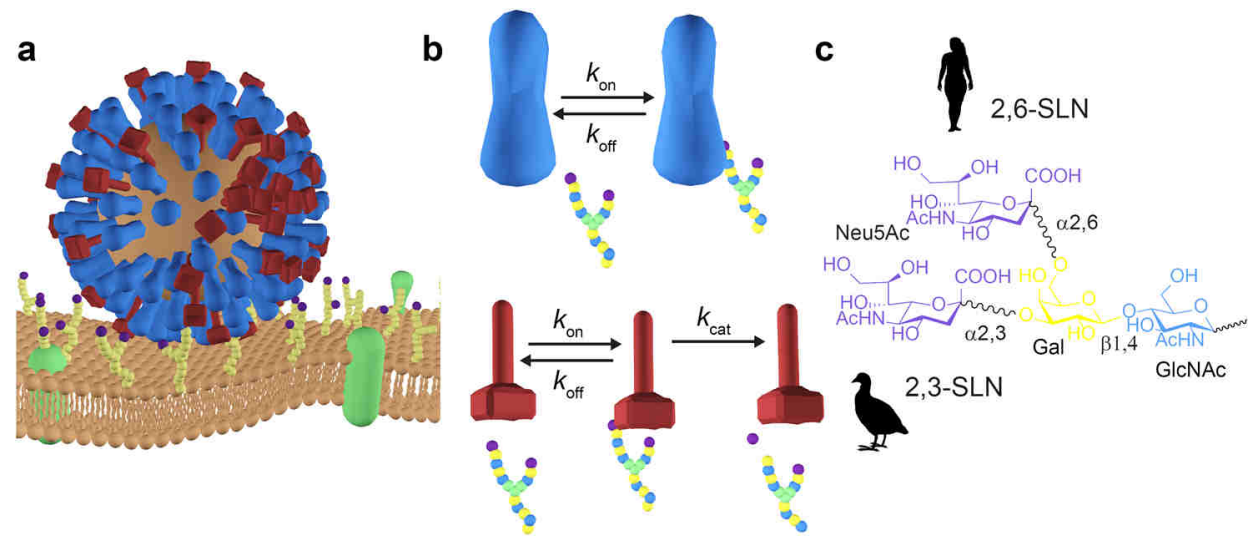

Figure 2.1 | Interactions of IAV with sialoglycan receptors. a) IAV binds to glycoproteins and glycolipids on a host cell membrane through its surface proteins HA (blue) and NA (red). The image is not to scale; the virus is $100-150 \mathrm{~nm},{ }^{7} \mathrm{HA}$ and NA are approximately 14 and $16 \mathrm{~nm}$ long, ${ }^{7}$ the lipid membrane is approximately $5 \mathrm{~nm}$ thick, ${ }^{12}$ glycans are 2-20 $\mathrm{nm},{ }^{10}$ mucins and cilia (Figure 2.5) are omitted. b) HA forms reversible interactions with sialoglycan receptors, whereas NA can bind the same receptors and cleave the sialic acid. c) Structure of the avian-type 2,3-SLN and human-type 2,6-SLN receptors. In the most abundant sialoglycans, the terminal sialic acid Neu5Ac is linked by either an $\alpha 2,3-$ or $\alpha 2,6$-linkage to the penultimate galactose. ${ }^{10}$ This linkage is hydrolyzed by NA. 
The high number and density of glycan-binding proteins on IAV make it a "multivalent particle", meaning that it has the potential to form multivalent interactions. Multivalent receptor-ligand interactions are reversible interactions between two particles (or a particle and a surface) that are constituted of multiple receptor-ligand pairs (Figure 2.2a). ${ }^{13}$ When a multivalent complex is formed, it is usually more stable than any of the contributing individual interaction pairs. In terms of kinetics, the dissociation rates of individual receptor-ligand complexes are usually the same when they are part of a multivalent complex as when they are not. Yet, the overall dissociation rate decreases because the probability that all contributing bonds unbind at the same time decreases with increasing numbers of interaction pairs. Moreover, when a tether between the ligands and receptors increases the local concentration, the association rate increases with it (Figure 2.2b). This local concentration is known as the effective molarity $E M$ and can be estimated from the volume that an unbound ligand in a partly associated complex can probe (Figure $2.2 \mathrm{c}$ ). ${ }^{14}$ The product of this effective molarity and the binding constant of the individual receptor-ligand interaction $K_{\mathrm{i}} E M$ is known as the multivalent enhancement factor, the amount by which each additional interaction increases the stability of a multivalent complex. The overall binding affinity or avidity constant of a multivalent interaction, which is the inverse of the dissociation constant $K_{\mathrm{D}}$, can be calculated by

$K_{a v}=K_{i}\left(K_{i} E M\right)^{n-1}$

where $n$ is the valency, i.e., the maximum number of interactions that can be formed simultaneously. ${ }^{15}$ This assumes that the binding sites are independent, i.e., no allosteric cooperativity between the binding sites occurs. ${ }^{14}$ 
a

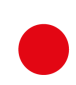

monovalent ligand

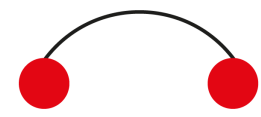

divalent ligand

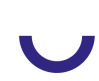

monovalent receptor

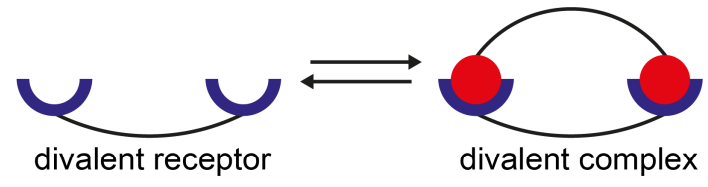

b

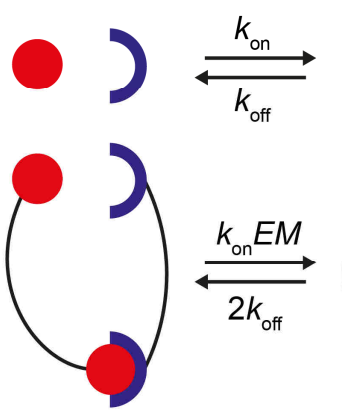

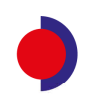

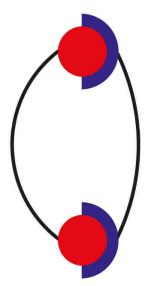

C

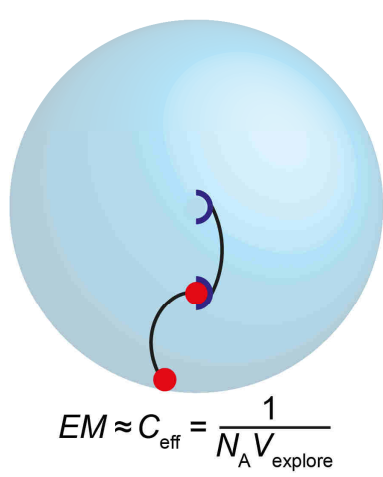

Figure 2.2| The differences between monovalent and multivalent interactions. a) The valency of receptor-ligand interactions is the number of connections that can be formed. b) A multivalent receptor-ligand equilibrium differs from a monovalent by an effective molarity $E M$ in place of solution concentrations. c) The effective molarity $E M$ can be approximated by estimating the volume that an unbound ligand in a partly bound complex can probe.

Multivalent interactions cause emergent properties, such as molecular walking and highly selective recognition, which are not obvious from the individual interactions or from viewing the overall stability of the complex. These properties arise from how multiple individual interactions act together, regarding energetics, structure, and dynamics. Most relevant for the discussion of IAV are: superselectivity, receptor recruitment and dynamicity (Figure 2.3). 
a
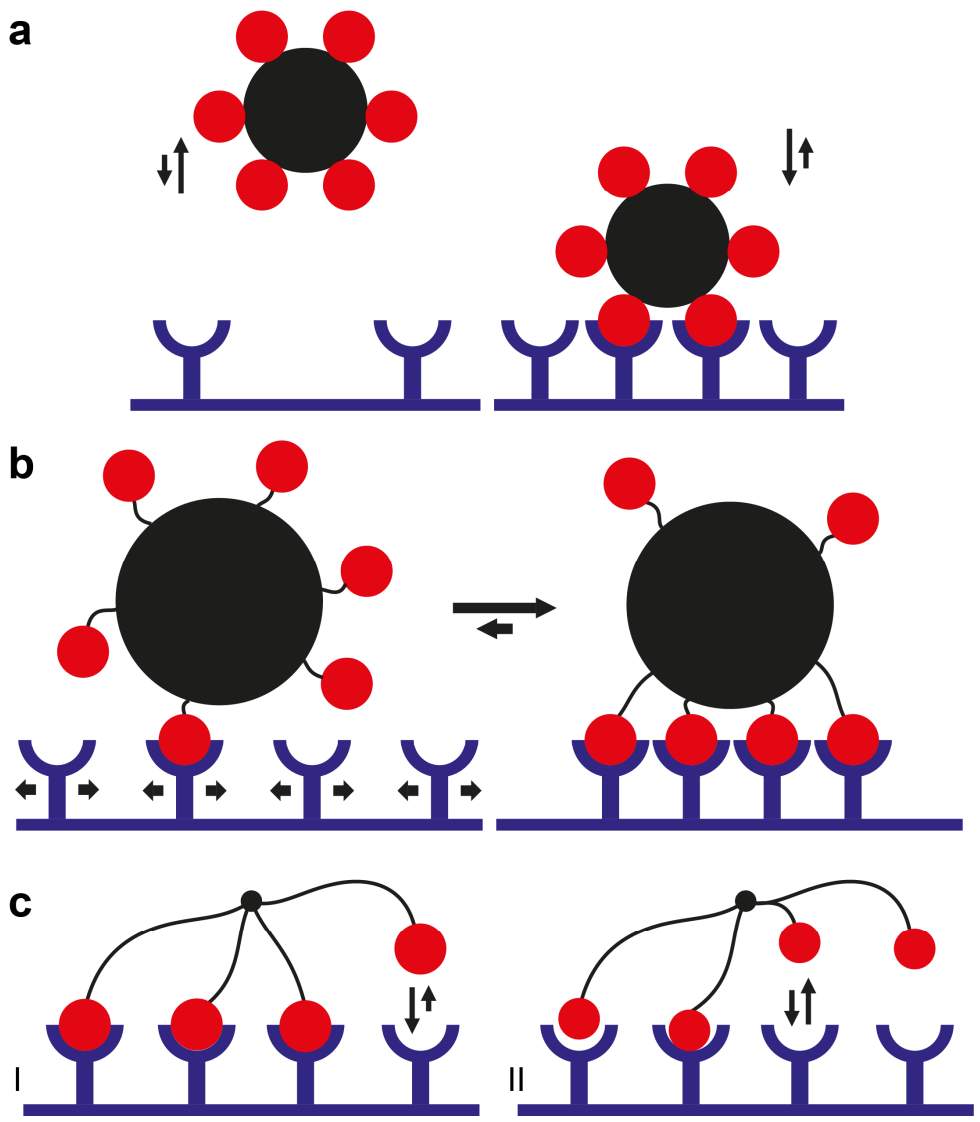

Figure 2.3 | Emergent properties of multivalent interactions. a) Superselectivity means that multivalent particles bind more strongly at receptor densities above a certain threshold value above which they can form sufficient numbers of simultaneous interactions. b) Receptor recruitment occurs when a multivalent particle binds to a surface with mobile receptors. When these bind to the particle, the receptors accumulate into the contact area, which results in a depletion of receptors in other areas. c) Dynamic complexes are possible with multivalent reversible receptor-ligand pairs. If the individual interactions are strong $(I)$, it is more likely that the complex returns to the same state after one receptor-ligand pair detaches than when the individual interactions are weak (II). This results in complexes that alternate between different bound states in which different receptor-ligand pairs are bound or unbound at any given moment. 


\subsubsection{Superselectivity}

Superselectivity of a particle with multiple ligands is the more than linear increase in binding with the density of receptors on a surface. ${ }^{16}$ For an equilibrium between monovalent ligands in solution and receptors on a surface, the number of bound ligands cannot increase more than linearly with the number of receptors, regardless of their affinity. But for multivalent particles that can bind multiple receptors simultaneously, their affinity depends exponentially on the number of interactions that can be formed (see Equation 2.1). Therefore, the fraction of bound particles is higher at high receptor densities, whereas at low receptor densities their binding is low and effectively monovalent (Figure 2.3a). This makes multivalent particles superselective towards higher receptor densities, and their selectivity can even approach "onoff" behavior.

Superselectivity can be applied for targeted delivery to cells with receptors that are not unique but instead are overexpressed compared to other cells. ${ }^{17-19}$ The receptor density at which the selectivity is highest can be tuned by varying the interaction strength and the architecture of the particle. ${ }^{20-22}$

The receptor density is directly related to the multivalent binding affinity (Chapter 4). The highest selectivity is achieved with a high number of interactions that are individually weak, with binding energies on the order of the thermal energy $k_{\mathrm{B}} T .{ }^{23}$ Additionally, even higher selectivities can be achieved when there are polymers on the particle or surface that give steric repulsion. ${ }^{24,25}$

We have developed a theoretical binding model based on the statistical thermodynamics of multivalent adsorption of IAV to a surface with short glycans (Chapter 4). The avidity constant is described by:

$K_{a v}=N_{A} V_{e x}\left(1+\frac{K_{i}}{N_{A} V_{\text {explore }}}\right)^{\tilde{\mathrm{N}}}$

where

$\tilde{\mathrm{N}}=A_{\text {contact }} \cdot \min \left(\sigma_{L}, \sigma_{R}\right)$

Here, $N_{A}$ is Avogadro's number, $V_{\text {ex }}$ is the volume excluded by a bound virus particle, $K_{\mathrm{i}}$ is the individual binding constant of a HA-glycan interaction, $V_{\text {explore }}$ is the volume accessible to a glycan, and $\tilde{N}$ is the average number of possible 
simultaneous interactions between $\mathrm{HA}$ on the virus and glycan receptors when the virus is adjacent to the surface. $A_{\text {contact }}$ is the area of the receptor surface that can be reached by $\mathrm{HA}$ on a surface-bound virus, and $\min \left(\sigma_{L}, \sigma_{R}\right)$ is the minimum of the density of receptors on the surface and that of receptor binding domains on the virus. The virus binding is superselective, because the avidity constant depends exponentially on the number of possible interactions.

\subsubsection{Receptor recruitment}

When a multivalent particle binds to mobile receptors (e.g. glycan-bound lipids present in a cell membrane or supported lipid bilayer, SLB), bound receptors remain associated with the particle as long as they are bound, whereas unbound receptors remain freely mobile. ${ }^{26}$ Because bound receptors need to unbind before they can move away from the particle, the contact area acts as a sink for diffusing receptors, leading to a higher local density of receptors inside the contact area and a lower density outside (Figure 2.3b). ${ }^{26,27}$ This clustering of receptors strongly increases the number of interactions for already bound particles as well as decreasing the number of available receptors for yet unbound particles. ${ }^{26,28}$ Depending on the density of receptors and ligands, recruitment may either increase or decrease the binding. ${ }^{29}$ This leads to a higher superselectivity and at lower receptor densities, ${ }^{29}$ but also to a lower maximum density of particles on the surface. ${ }^{26,28}$ If both the receptors on the surface and the ligands on the particle are mobile, recruitment may occur on both sides..$^{26,28}$ Recruitment of ligands can enhance the recruitment of receptors because it increases the number of ligands that are available. ${ }^{26}$ If sufficient receptor-ligand pairs are involved, recruitment of receptors and ligands can form a kinetic trap for otherwise reversible interactions. ${ }^{28}$

\subsubsection{Dynamic complexes}

The third emergent property of multivalent complexes is that they are dynamic. If the concentration of a multivalent particle is higher than its dissociation constant, the stable state of the particle is bound, even though the bound state may not be the favored state for its ligands individually (Figure 2.3c). For a multivalent complex with short tethers and receptor-ligand pairs of moderate affinity, such as cyclodextrin and adamantane, $K_{i} E M$ can be easily as high as $10^{4} .{ }^{30}$ In such systems, the fully bound complex is the most probable state and simultaneous unbinding of more receptor-ligand pairs in a complex becomes 
increasingly improbable. When the multivalent enhancement factor $K_{\mathrm{i}} E M$ is between 0.1 and 10 , between 10 and $90 \%$ of ligands are bound at equilibrium. ${ }^{31}$ We call such systems "weakly multivalent". In a weakly multivalent system, all receptor-ligand pairs exchange rapidly and reside for significant fractions of the time in both the bound and unbound states. As a result, there is a significant probability that one or more receptor-ligand pairs in the complex are unbound at any given time, while the probability of complete unbinding of the complex remains low due to a relatively high number of interactions.

This combination of stability and dynamicity is called on by molecular walkers. A molecular walker is a particle with "feet" that can bind to and release from a surface sequentially. Examples of molecular walkers include the biological walkers kinesin and myosin $\mathrm{V}$ motor enzymes that transport cargo along microtubules and actin filaments, ${ }^{32-34}$ as well as many different synthetic walkers. ${ }^{35}$ Such walkers must be multivalent because by definition one "leg" must remain bound when other legs detach to make a step. To allow lateral mobility, the individual interactions must be sufficiently weak to allow the walker to step to an adjacent receptor, otherwise the walker is kinetically trapped. ${ }^{36}$ High surface-restricted mobility is achieved with a high number of weakly multivalent interactions. ${ }^{37}$

The multivalent enhancement factor $K_{\mathrm{i}} E M$ of influenza $\mathrm{HA}$ is estimated to be 512 , making it weakly multivalent. ${ }^{31}$ Despite its weak interactions, IAV binds irreversibly to the surface of cells when its NA is inhibited. ${ }^{38}$ The weakly multivalent nature allows the exchange of receptors from HA to NA, which allows the virus to detach from a surface after off-target binding. ${ }^{6}$ Because IAV is both weakly multivalent and superselective, minor changes in the number of interactions or their individual affinity can drastically affect virus binding. Combined with the intrinsic structural variability of the virus, this allows efficient adaptation of the virus to environmental pressure, causing escape from immunity and loss of drug responsivity. 8,39 


\section{Chapter 2}

a

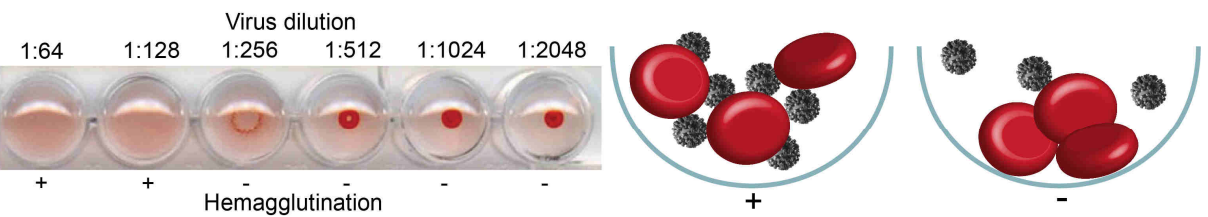

b

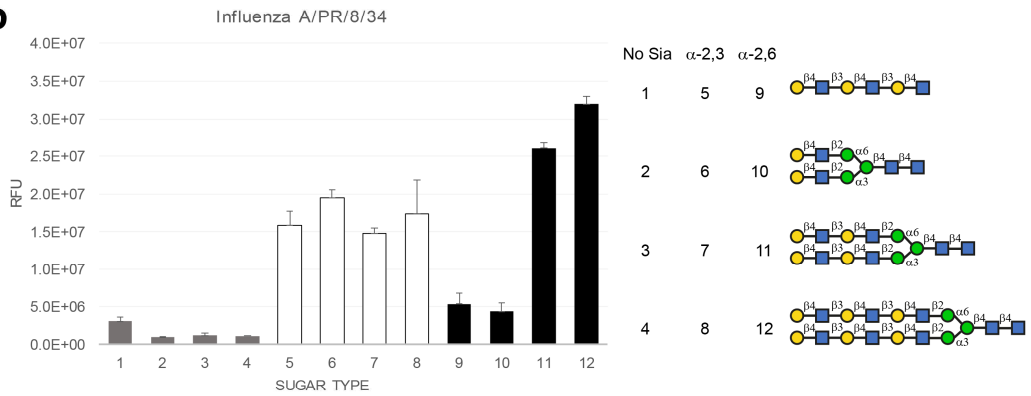

C

Step 1 Step 2
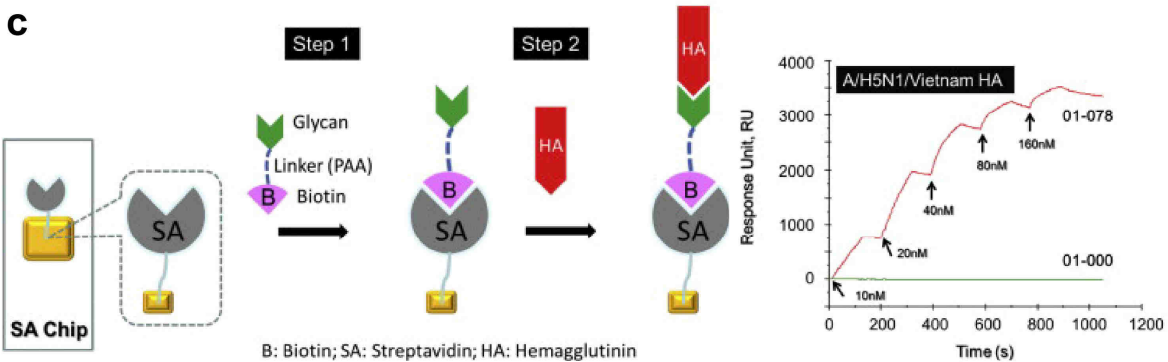

\section{d}

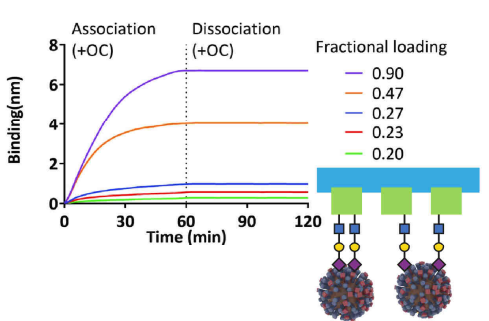

e

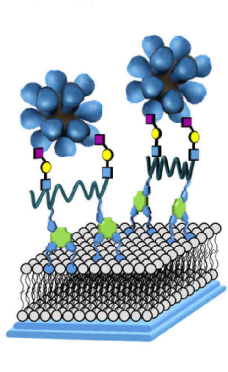

f

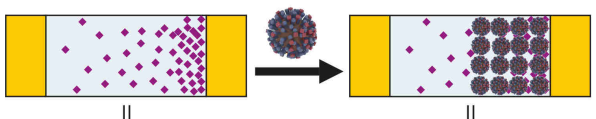

Glycans
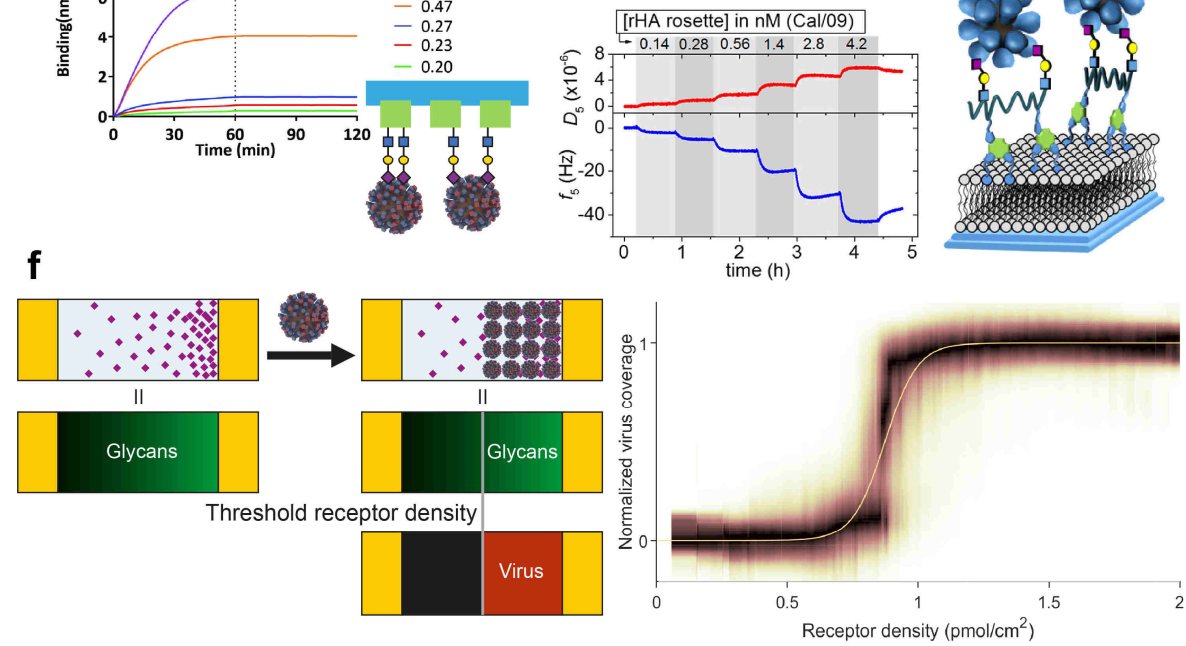
A dynamic, supramolecular view on the multivalent virus-host cell interaction

Figure 2.4| Overview of techniques to measure the multivalent binding of IAV. a) Example of the result of a hemagglutination assay and schematic representation of agglutinated and nonagglutinated red blood cells in the presence of a virus. The titer in this example is $128 \mathrm{HA}$ units. Reproduced with permission under CC BY $4.0 .{ }^{40}$ Copyright 2012, Public Library of Sciences. b) Example of virus binding data in relative fluorescence units (RFU) for a glycan array of 12 glycans (Chapter 4). c) Example of a surface architecture for measuring influenza HA binding with SPR and results from a titration with HA. A polymeric scaffold with grafted glycans and biotin is immobilized on a commercial streptavidin-modified gold chip (Step 1). The SPR response is measured while increasing concentrations of HA are passed over the chip (Step 2). Reproduced with permission. ${ }^{41}$ Copyright 2011, Elsevier B.V. d) Example of a surface architecture for measuring the binding of IAVs with $\mathrm{BLI}$ and results from a titration with fractional loading of glycans. Biotinylated glycans are loaded onto commercial streptavidin-coated biosensor tips while measuring the level of loading to control the receptor density. A titration is performed by measuring the virus binding to an array of biosensor tips with various levels of glycan loading. Reproduced with permission under CC BY 4.0. ${ }^{42}$ Copyright 2019, Public Library of Sciences. e) Example of a surface architecture for measuring the binding of HA rosettes with QCM-D and results from a titration with rosettes. A supported lipid bilayer containing biotinylated lipids is formed on a $\mathrm{SiO}_{2}$ coated QCM chip. Streptavidin and a polymeric scaffold with grafted glycans and biotin are subsequently immobilized. The frequency shift and dissipation are measured while increasing concentrations of HA rosettes are passed over the chip. Reproduced with permission under CC-BY-NC-ND 4.0. ${ }^{31}$ Copyright 2019, American Chemical Society. f) Schematic representation of how the threshold receptor density is determined in multivalent affinity profiling, and an example of a virus binding profile as function of receptor density. The virus binding profile shows Equation 2.2 fitted (yellow S-curve) over a density map of data points ( $\mathrm{N} \approx 400,000$ from 3 pairs of micrographs) (Chapter 4 ).

\subsection{Techniques to measure the binding of influenza viruses}

Because receptor recognition by IAV is considered a key determinant in host specificity of the virus and an indicator for zoonotic potential, ${ }^{2,43-45}$ several techniques have been developed to measure the receptor specificity of influenza HA or its avidity for a receptor. To unravel the contribution of individual HAs to the multivalent binding of IAV, titrations in solution are used. In this case, the binding is measured with techniques such as NMR and microscale thermophoresis (MST), ${ }^{46,47}$ but we will not discuss these here further. 


\subsubsection{Hemagglutination assay}

The hemagglutination assay (Figure 2.4a) is commonly used as a rough estimate of virus concentration and avidity. It is performed by mixing chicken red blood cells with a serial dilution of the virus and observing until what dilution the virus can agglutinate the red blood cells. ${ }^{48,49}$ The highest dilution at which no agglutination takes place is the "titer", a concentration that depends on the avidity of the virus. To study receptor specificity, the red blood cells are treated with sialidases that are specific for either 2,3-SLN or 2,6-SLN before adding the virus solutions..$^{50}$ Specificity is observed when the virus agglutinates $2,3-\mathrm{SLN}$ or 2,6-SLN at higher dilutions than the other. The assay is simple and does not require specialized equipment, but the output is not easily translated into biophysical properties, and the glycan structure of chicken red blood cells is not representative for either mammalian lungs or avian intestines. ${ }^{51}$ The differences in glycan structures show clearly in seasonal IAV H3N2, which has lost its ability to agglutinate chicken red blood cells by prolonged adaptation to the human population so that cells from turkeys and guinea pigs are used instead. ${ }^{52}$

\subsubsection{Glycan microarrays}

Glycan microarrays are small islands of different glycan types that are printed on a surface in an array so that the binding to each glycan type can be measured relative to the others (Figure 2.4b). With glycan microarrays, the receptor specificity can be further analyzed to account for the wide variety of glycans that exists on cells. ${ }^{53} \mathrm{~A}$ library of glycans ranging from several to hundreds of different glycans is printed on amine-reactive glass. ${ }^{54,55}$ These glycans can be synthetic or obtained from natural sources. ${ }^{56}$ The binding of HA is quantified by conjugating recombinant HA trimers to fluorescently labelled antibodies and measuring the fluorescence intensity at each glycan spot. ${ }^{57}$ This technique has proved a powerful tool to study the evolution of receptor specificity. ${ }^{58,59}$ The significance of knowing the HA specificity for synthetic glycans is limited by our understanding of the glycan structure of influenza host cells. ${ }^{57,60}$ For that reason, shot gun microarrays use HPLC-purified glycans that are released from host tissue. ${ }^{61}$ This combination of glycomics and virus binding studies has revealed previously unknown receptors of IAV ${ }^{62}$ As another way to improve glycan arrays, it has been suggested that the architecture of the glycocalyx is better represented when the glycans are bound to polymer chains rather than 
A dynamic, supramolecular view on the multivalent virus-host cell interaction

flat surfaces. ${ }^{63} \mathrm{~A}$ more representative presentation of $\mathrm{HA}$ is achieved when whole viruses are used instead of recombinant HA constructs (Chapter 4).

\subsubsection{Surface plasmon resonance}

For quantitative measurements of the affinity of influenza HA to cell surface mimics, surface plasmon resonance (SPR) can be used (Figure 2.4c). Suenaga et al. modified commercial streptavidin-coated gold chips with a polymeric scaffold with grafted glycans and biotin to create a receptor surface. ${ }^{41}$ They titrated recombinant $\mathrm{HA}$ to this chip and measured the response to determine the $K_{\mathrm{D}}$ of the trimeric HA. Because this technique aspires to be quantitative, it is important that the surface architecture and presentation of HA are representative. Because they used a densely modified surface, this method does not account for changes in avidity with receptor density. It is therefore important to combine such SPR studies with substrate chemistries that allow receptor density variation. ${ }^{64}$

\subsubsection{Biolayer interferometry}

Biolayer interferometry (BLI) uses a fixed concentration of virus and varies the glycan density on the surface (Figure $2.4 \mathrm{~d}$ ) ${ }^{46,47}$ The virus binding is measured by the wavelength shift in the interference pattern of white light reflected from the surface of a biosensor tip. The biosensor tip with glycans is suspended into a virus solution. A BLI machine can process $96-$ or 384-well sample plates, conveniently allowing measurements with varied surface modification. The biosensor tips are usually functionalized with streptavidin. Receptors can be biotinylated monovalent glycans, polymers with grafted glycans, or glycoproteins. ${ }^{42,46}$ The density of receptors can be controlled by varying the loading time or concentration, ${ }^{42,46}$ or by diluting the receptors with a dummy receptor (Chapter 4 ). The resulting binding profiles as a function of relative sugar loading are used as a relative measure of virus avidity. When a virus is titrated to determine $K_{\mathrm{D}}$, values can differ by two orders of magnitude, depending on the relative sugar loading. ${ }^{5}$

\subsubsection{QCM-D}

To mimic the structure of a cell membrane, we developed a method to display glycans on supported lipid bilayers (Figure 2.4e). ${ }^{31}$ We mixed antifouling zwitterionic lipids with a small percentage of biotinylated lipids, and 
subsequently immobilized streptavidin and biotinylated glycans to obtain a well-defined surface that mimics a membrane with glycoproteins and retains some lateral mobility of the receptors. We used quartz crystal microbalance with dissipation monitoring (QCM-D) to monitor the surface modification and measure the virus binding. The QCM-D measures binding and the stiffness of bound layers by frequency and dissipation shifts in a resonating quartz crystal with a modified surface inside a flow cell. With this technique, we studied the binding of multivalent recombinant HA nanoparticles to glycans on a polymer backbone that was bound onto a supported lipid bilayer with lateral mobility. The glycan-modified polymers were displayed on the surface in varying densities. Because the HA nanoparticles were closer in size to the glycanmodified polymers than to IAV, we saw that the local glycan density rather than the average glycan density determined the $K_{\mathrm{av}}$. When biotinylated monovalent glycans and whole viruses were used instead, we observed the superselective binding of IAV. ${ }^{65}$

\subsubsection{Multivalent affinity profiling}

More recently, we developed a method called "multivalent affinity profiling" (MAP) that uses the superselectivity of IAV binding to quantify the avidity of IAV by measuring its binding profile on receptor density gradients (Figure 2.4f, Chapter 4). The receptor density gradients were prepared by immobilizing streptavidin and biotinylated glycans on biotinylated lipids in gel-state supported lipid bilayers. These biotinylated lipids were arranged in an electrophoretic gradient using a microfluidic device that was designed for that purpose. ${ }^{66,67}$

We used fluorescent dye-labelled virus and streptavidin (which signals the presence of receptors) and determined their colocalization with fluorescence microscopy, from which we obtained virus binding profiles as a function of receptor density. The superselective binding profile exhibits a step at the threshold receptor density. The threshold receptor density is related to the avidity by Equation 2.2 and is the point where $K_{\text {av }} \cdot[$ virus] $=1$. We could estimate the number of interactions and their individual contribution to the avidity by fitting Equation 2.2 to the virus binding profile. This technique allows a complete receptor density titration in a single pair of micrographs. In a pair of micrographs, the fluorescence intensities of each pixel represent a datapoint of 
A dynamic, supramolecular view on the multivalent virus-host cell interaction

virus binding and receptor density. Because the fluorescence intensity is normalized, data from multiple areas in a micrograph, multiple micrographs and multiple experiments can be combined to improve the resolution. This makes it an especially powerful technique in comparison to other techniques that require separate experimental steps for each data point.

\subsection{Dynamic interactions during initial stages of infection}

We have described how the structure and receptor-ligand interactions of IAV make it a weakly multivalent nanoparticle, and how multivalent binding can lead to superselectivity, receptor recruitment and the formation of complexes that retain dynamic properties. We then discussed several techniques that have been used to measure the multivalent binding of IAV. In this section, we will first discuss how NA controls the binding of the virus and how this can be measured. Then we will discuss the role of multivalent interactions and the HA/NA functional balance during the passage of IAV through the mucus and across the cell surface, concluding with how the physical process of multivalent binding can elicit a biological response from the host cell that leads to endocytosis of the virus.

\subsubsection{The influence of NA on binding}

In the initial stages of influenza infection, both $\mathrm{HA}$ and NA play a role in receptor recognition. HA binds receptors, and NA binds and cleaves. In the absence of catalytic activity, NA can bind sialic acid with similar or even higher affinity compared to HA. ${ }^{68,69}$ The receptor-binding function and the receptor-cleaving function of these two glycoproteins must be balanced for efficient transmission and proliferation of the virus. ${ }^{3,70,71}$ This functional balance is also reflected in the evolution of HA and NA. ${ }^{72-76}$ Likely, when a mutation in HA or NA affects their binding or cleaving function, the restoration of this balance forms a driving force in the adaptation of the other. ${ }^{72,73,77,78}$ In-vivo studies showed that both lower NA activity and stronger binding by HA can lead to less efficient virus replication. ${ }^{79}$ If NA is too active, it prevents viruses from binding to their host cell, whereas too low NA activity leads to virus aggregation and entrapment of progeny viruses on the surface of host cells. ${ }^{2,3,80}$ 

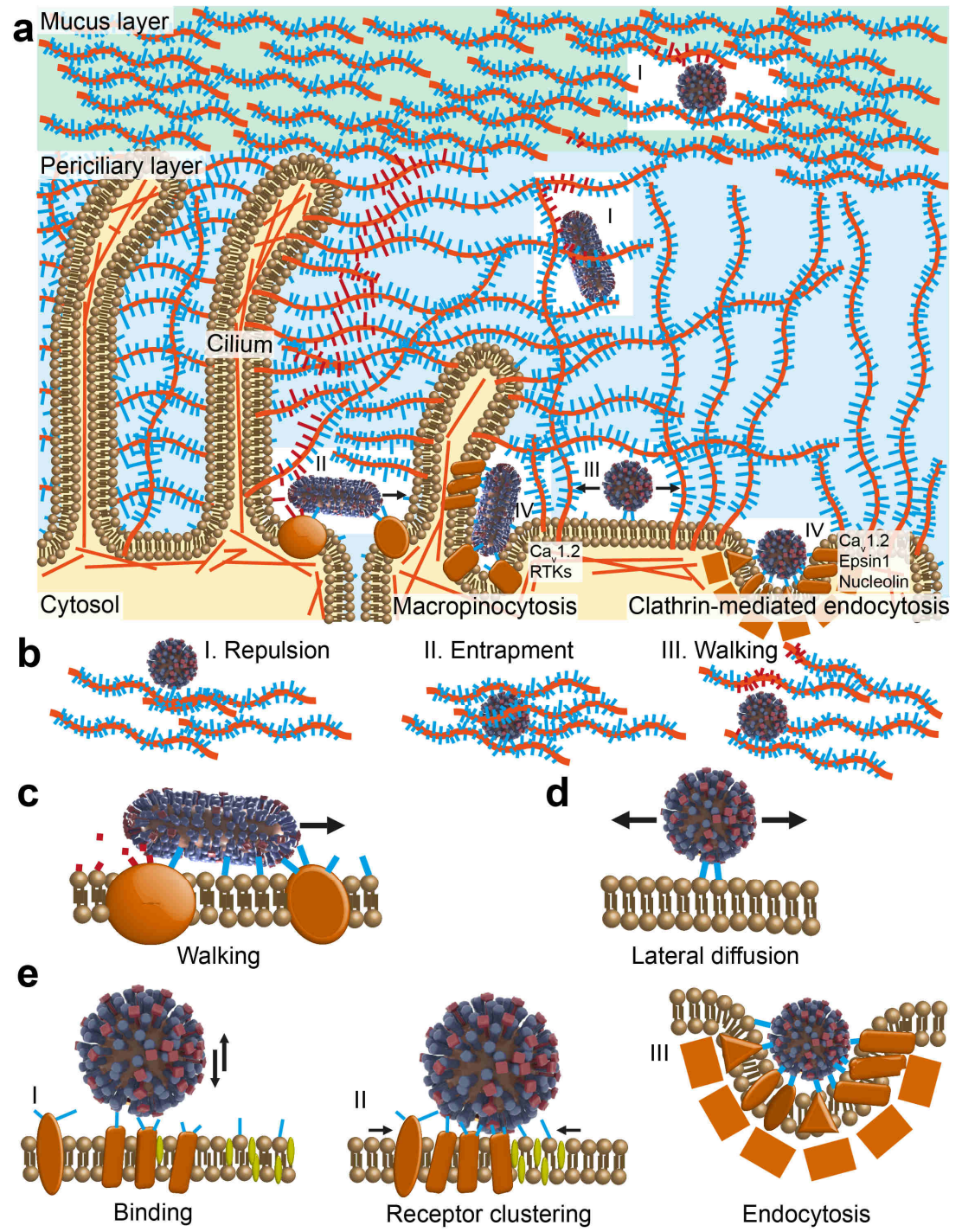

Figure 2.5 Structure of the mucus and overview of the mechanisms that IAV use during the initial stages of infection. a) Structure of the mucus in human airways. ${ }^{81,82}$ Shown are one ciliated cell and one non-ciliated cell. IAV crosses the mucus layer and periciliary layer by receptor-cleaving molecular walking (I). Cleaved glycans that mark the path of the virus are shown in red. Then the viruses move along the surface of the cells by a combination of molecular walking (II) and lateral diffusion with receptors (III). Endocytosis (IV) takes place by either macropinocytosis, which depends on voltagedependent calcium channel Cav1.2 and receptor tyrosine kinases (RTKs), or by clathrin- 
mediated endocytosis, which depends on Cav1.2, Epsin1 and nucleolin. b) Active motility is needed to cross the mucus and periciliary layer. I. When there is no interaction, particles are repelled by the charged brush. II. Particles that have an affinity for sialic acid are entrapped. III. IAV, which binds and cleaves sialic acid, can walk over the mucins. c) On the cell surface, IAV can use its receptor-cleaving molecular walk to move across immobile areas or from cell to cell. d) IAV can diffuse laterally with mobile receptors in fluidic membranes. e) Model of how multivalent interactions induce cellular uptake. I. The functional balance of receptor binding and cleaving prevents kinetic traps but allows a long enough residence time at locations suitable for cell entry. II. A bound IAV can bind to additional receptors that diffuse laterally in the lipid membrane so that they accumulate in its contact area. III. The clustering of glycolipids and some membrane glycoproteins can induce membrane curvature, whereas other membrane glycoproteins are activated by clustering and act as a trigger for the endocytic machinery of the host cell. IAV is taken up by either macropinocytosis or clathrin-mediated endocytosis. Both pathways may be triggered by recruitment of Cav1.2 elsewhere on the cell surface.

In most techniques that assess the multivalent binding of IAV, the influence of NA on the virus binding is ignored as they require either the use of isolated HA or addition of an NA inhibitor. Benton et al. showed that BLI could also be used to measure the time-dependent binding of IAV in the absence of NA inhibitor to determine the residence time of the virus when allowed to cleave its receptors. ${ }^{5}$ The residence time measured in this way could be used to assess the HA/NA balance of a virus strain on different receptor types. $5,42,83$ Additionally, it was shown that NA can contribute to the initial binding rate of viruses. ${ }^{42,83}$ This is partly due to the substrate binding at the catalytic site, and partly to the presence of a second sialic acid-binding site on NA next to the catalytic site, which is also affected by NA inhibitors and contributes to the catalytic activity of NA. ${ }^{42,83-85}$

\subsubsection{Binding and cleaving in the mucus}

The role of NA is especially important in the mucus, which acts as the first barrier of a potential host against respiratory pathogens such as IAV. The mucus consists of a mucus layer on top of the periciliary layer (Figure 2.5a). ${ }^{81}$ The mucus layer is a dense gel of high molecular weight proteins called "mucins", which are decorated with $50-80$ wt\% of glycans that are rich in sialic acid. ${ }^{86}$ This gel is propelled by cilia that extend from the epithelial cells of the respiratory tract. ${ }^{81,87}$ It can therefore easily entrap and remove sialic-acid binding viruses 
such as IAV. ${ }^{88}$ To prevent this entrapment and clearance by the mucus, influenza NA cleaves the sialic acid when the virus binds to mucins. In the human airways, mucins are rich in 2,3-SLN, whereas the cells underneath express more 2,6SLN. ${ }^{89}$ The HA/NA balance of human IAV, therefore, favors cleaving of 2,3-SLN but binding to $2,6-\mathrm{SLN}$. $^{1,3,90}$

Recently, Vahey and Fletcher demonstrated another function of this HA/NA balance. ${ }^{6}$ They showed that the asymmetric organization of HA and NA on filamentous IAV imparts directional motility in mucus, which would help the virus to cross the mucus (Figure $2.5 \mathrm{a}$ and $\mathrm{b}$ ). However, such motility has also been observed in egg-adapted IAV X-31 and PR8 strains, ${ }^{42,91}$ which are highly spherical ${ }^{92}$ so that the binding and cleaving functions are less separated. Therefore, we compared the motility of IAV with the behavior of artificial and simulated molecular walkers and argued that directional motility is intrinsic to receptor-cleaving multivalent particles, rather than a result of asymmetric organization alone. ${ }^{93}$ The enhancement of directionality by asymmetric organization in filamentous viruses would allow motility over longer distances, which is consistent with the observation that the filamentous phenotype is more abundant in patient samples than in viruses from eggs or in cell cultures without mucus. ${ }^{94}$

Underneath the mucus layer, the periciliary layer forms an even stricter barrier. It was shown that while the mucus permits diffusion of inert particles of the size of a virus, the periciliary layer excludes even $40 \mathrm{~nm}$ particles. ${ }^{81,88}$ This barrier is formed by tethered mucins that extend in a bottle-brush shape from the cilia. ${ }^{82}$ Simply cleaving the sialic acids that would entrap the virus, would not undo the steric protection. We and others proposed therefore that IAV must use an active molecular walk to cross the periciliary layer (Figure 2.5b). ${ }^{93,95}$

\subsubsection{Dynamic interactions of influenza on host cells}

After having crossed the mucus layer and periciliary layer, an IAV must find a suitable place for endocytosis on a suitable host cell. The favored cell types differ between IAVs, with highly pathogenic avian IAV infecting in humans primarily the lower airways and human seasonal IAV infecting the upper airways. ${ }^{96,97}$ In tracheal and bronchial cell cultures, most human IAVs and some avian favor non-ciliated cells over ciliated cells, ${ }^{98,99}$ which was initially attributed to the presence of more $2,3-\mathrm{SLN}$ on ciliated cells and more $2,6-\mathrm{SLN}$ on non- 
A dynamic, supramolecular view on the multivalent virus-host cell interaction

ciliated cells. ${ }^{98}$ But this idea is challenged in more recent literature that claims a more uniform distribution of 2,3-SLN and 2,6-SLN over the different cell types. ${ }^{2}$ Additionally, Richard et al. found that human rather than avian IAVs bound readily to the ciliated cells of nasal epithelial tissue culture, ${ }^{100}$ and Davis et al. found that mutants of the 1918 pandemic IAV bound and infected bronchial epithelial cells comparably, regardless of their preference for 2,3-SLN or 2,6-SLN receptors. ${ }^{101}$

Sakai et al. suggested that IAV is unlikely to enter the cilia themselves, because they are full of microtubules and their diameter is only slightly larger than a virus (Figure 2.5a)..$^{91}$ Therefore, IAV must cross the full depth of the periciliary layer to reach the cells underneath. A preference for non-ciliated cells then suggests that some IAVs cross from cell to cell by moving from receptor to receptor. The receptor-cleaving molecular walk that allows IAV to move through the mucus may also provide a method to move efficiently across the surface of the cells (Figure 2.5c).

Because cell membranes are largely fluidic, they allow lateral diffusion of receptors (Figure $2.5 \mathrm{~d}$ ). The diffusion coefficient of a membrane-bound particle decreases with the number of receptors that bind to the particle. ${ }^{102}$ Müller et al. studied the relationship between mobility and dissociation of IAV on SLBS with up to $1 \%$ glycolipids. ${ }^{103}$ They found a peak in the dissociation rate constant $k_{\text {off }}$ for viruses with a diffusion constant of around $0.2 \mu \mathrm{m}^{2} / \mathrm{s}$, suggesting that NA has the strongest effect on viruses that formed neither too many nor too few interactions. In their work on directional motility of IAV, Vahey and Fletcher simulated a virus on a surface with mobile receptors and also found an optimum in the directional bias for moderate diffusion coefficients of sialic acid. ${ }^{6}$ While it is tempting to compare numbers, these systems are too different to draw conclusions beyond the existence of an interplay of lateral mobility and HA/NA balance.

\subsubsection{Recognition and endocytosis through multivalent interactions} When an IAV meets a suitable location for endocytosis, it can enter the host cell via multiple pathways. Macropinocytosis is the primary pathway for filamentous IAV and accounts for $35 \%$ of the uptake of spherical virions (Figure 2.5a). ${ }^{104,105}$ The remaining $65 \%$ of the uptake of spherical virions is accounted for by clathrin-mediated endocytosis. ${ }^{104}$ Interestingly, if the clathrin-mediated 
pathway was inhibited, macropinocytosis increased such that the effect on the total virus uptake was limited. ${ }^{106-108}$

Early drawings suggest that the multivalent binding of IAV to a cell leads to a progressively increasing number of interactions that deform the membrane and thus trigger endocytosis through the physical deformation. ${ }^{13}$ But even for a simplified system where the receptor-ligand interactions are strong, the number of interactions and membrane deformation find a balance due to the mobility of receptors. ${ }^{109}$ Therefore, SV40, a virus that binds glycolipids with much higher affinity than IAV, relies on lipid phase separation as a result of receptor recruitment to induce sufficient membrane deformation for cell entry. ${ }^{110}$ For IAV, such strong deformations appear unfavorable, as cryo-TEM micrographs of bound viruses show that IAVs are largely wrapped by the membrane in the presence of NA inhibitor, but induce little membrane deformation without NA inhibitor. ${ }^{111}$ As physical deformation of the membrane appears insufficient to trigger endocytosis, there must be a trigger that induces a biological response to the binding of IAV, such as an entry receptor. Several glycoproteins appear to be involved in the endocytosis of IAV, but most are not essential, suggesting that there is redundancy in the receptors as well as the pathways. A shared feature of these glycoproteins is that they may be clustered upon multivalent binding to IAV.

Epsin and nucleolin are involved in clathrin-mediated endocytosis

Clathrin-mediated endocytosis does not rely on existing clathrin-coated pits or places where they form frequently. ${ }^{104}$ Instead, the clathrin-coated pits where IAV is taken up form newly upon the binding of viruses. This suggests that the endocytosis of IAV requires the presence of specific biomolecules that both interact with IAV and induce the formation of clathrin-coated pits. The protein epsin 1, which plays a role in membrane deformation, was identified as a cargospecific receptor for IAV that could initiate the formation of clathrin-coated pits. ${ }^{106}$ When epsin 1 was knocked down by $87 \%$, the fraction of viruses that used the clathrin-mediated pathway decreased by $68 \%$. Another protein, nucleolin, which is expressed ubiquitously and plays a role in molecular transport in cells, was also observed to bind IAV, and its knockdown led to inhibition of clathrin-mediated endocytosis. ${ }^{107}$ On the cell surface, nucleolin 
A dynamic, supramolecular view on the multivalent virus-host cell interaction

displays both 2,3-SLN and 2,6-SLN suggesting that it has the capacity to be recruited by IAV (Figure 2.5e). ${ }^{112}$

RTKs are involved in macropinocytosis

Eierhof et al. showed that IAV uses several receptor tyrosine kinases (RTKs) for cell entry, which are a family of transmembrane receptor proteins that play a role in regulating cellular processes by dimerization upon binding specific ligands. ${ }^{113}$ The multivalent binding of the virus to sialic acid on these receptors induces clustering and activates the RTKs which undergo cell entry together with the virus ${ }^{113}$ by macropinocytosis. ${ }^{108}$

\section{Clustering of Cav1.2 activates both pathways}

Another glycoprotein that plays a role in both pathways was identified by Fujioko et al. who found that IAV induced oscillations in cytosolic $\mathrm{Ca}^{2+}$ concentrations that activated both clathrin-mediated and clathrin-independent endocytosis. ${ }^{114}$ They identified a sialylated voltage-dependent calcium channel $\left(C_{v} 1.2\right)$ that is responsible for these oscillations. ${ }^{115}$ These channels are organized in the membrane in clusters of eight that work cooperatively and are activated through clustering by intracellular $\mathrm{Ca}^{2+}$ that binds to the $\mathrm{C}$-termini of these proteins. ${ }^{116} \mathrm{~A}$ similar activation may be induced by IAV when it binds to sialic acid on multiple channels of a cluster simultaneously (Figure 2.5e). Fujioko et al. did not report whether Cav1.2 is taken up together with the virus. ${ }^{115}$ Because $\mathrm{Ca}^{2+}$ spreads over the whole cell during these virus-induced oscillations, endocytosis may be upregulated anywhere on the cell so that $\mathrm{Ca}_{v} 1.2$ does not necessarily act as an entry receptor but may trigger the uptake of viruses elsewhere on the cell membrane.

Other cell membrane components may contribute to avidity

Sialic acid groups that are displayed on membrane glycoproteins appear pivotal in the recruitment of endocytic machinery, but other membrane components may also play a role in efficient uptake of IAV. Chu and Whittaker showed that $\mathrm{N}$-linked sialoglycans are required for cell entry of IAV, ${ }^{117}$ but De Vries et al. showed that in absence of competition from serum components IAV could enter cells that only had O-linked glycans and glycolipids. ${ }^{118}$ Eierhof et al. found that the RTKs responsible for cell entry of IAV were associated with lipid raft domains, which are rich in cholesterol and sialic acid-displaying ganglioside GM1. ${ }^{113}$ Removal of cholesterol with methyl- $\beta$-cyclodextrin impaired virus 
uptake. Conversely, it was shown that cholesterol could cluster gangliosides to provide areas of higher local receptor density, which increases the binding of IAV. ${ }^{119}$ Cells without gangliosides can be infected by IAV, but less efficiently. ${ }^{120}$ Byrd-Leotis et al. showed that human IAVs bound not only to sialic acid, but also to phosphorylated high-mannose glycans, presumably using a different receptor binding site. ${ }^{62}$ These additional attachment points may provide additional avidity and longer residence time for a virus during the recruitment of its entry receptors. The irreversible binding of viruses in the presence of NA inhibitors suggests that these additional attachment points can also block the recruitment of entry receptors. ${ }^{111}$ The selectivity of NA may therefore be an important factor in maintaining a functional balance that allows a virus to remain bound on a cell surface while sampling different receptors. Here, the linkage of the sialoglycans is again important as BLI studies showed that the residence time on 2,6-SLN is often dramatically longer than on 2,3-SLN. ${ }^{5,83}$

\subsection{Conclusions}

Multivalency may be best known for the increase in binding strength, but in a biological context, it plays a role in superselective binding, recruitment of receptors and the formation of dynamic complexes. The example of IAV shows that (super)selectivity and maintaining dynamicity may be even more important in living systems than binding strength. The combined actions of HA and NA ensure that viruses are not trapped in off-target binding.

A range of techniques and surface chemistries are available to assess the avidity and receptor specificity of $\mathrm{HA}$, varying from the hemagglutination assay, which uses blood cells, and glycan microarrays, which use a wide range of glycans immobilized on glass, to multivalent affinity profiling, which uses glycans in a receptor density gradient on supported lipid bilayers. Because the way receptors are presented determines the observed avidity and specificity, the selection of a representative surface chemistry is key to understanding the IAVglycan interactions at a molecular level. Although the importance of the functional balance between binding and cleaving is widely acknowledged, only a few studies include NA in IAV binding assays. Avidity and specificity are quantified by $K_{\mathrm{av}}$, threshold receptor density or relative binding. To quantify the functional balance of binding and cleaving, the residence time and the initial binding rate appear promising parameters. 
A dynamic, supramolecular view on the multivalent virus-host cell interaction

The ability of IAV to form transient complexes of multiple weak interactions allows it to efficiently cross the mucus barrier and to use redundant cell entry pathways by binding to common glycan motifs. We propose that the virusinduced receptor clustering of membrane glycoproteins, in particular Cav1.2, translates the physical effect of multivalent binding into a biological signal that leads to virus uptake by the host cell. The HA/NA functional balance may also control the recruitment of receptors for endocytosis.

The multivalency of IAV is key to understanding its biophysical properties. The techniques that are used to evaluate the multivalent binding of IAV may be improved by attuning the surface chemistry of sensors to the presentation of glycans in the glycocalyx. Because both the role and presentation of decoy glycans and glycans that participate in endocytosis differ, different surface chemistries may be needed to answer different scientific questions. Because NA has a role in the selectivity of IAV, assays that include NA may be more predictive for host specificity and tissue tropism than traditional assays. A better understanding of how IAV traverses the mucus, binds glycans, and enters host cells can help in the development of antivirals. ${ }^{121}$ The redundancy in cell entry pathways of IAV forms a challenge for blocking cell entry against which combination therapies may be effective. ${ }^{122,123}$ Therefore, multivalent virus inhibitors that block the binding of IAV and interfere with its transport in the mucus may be still more effective. ${ }^{13,124,125}$

\subsection{References}

1. Byrd-Leotis, L., Cummings, R. D. \& Steinhauer, D. A. The Interplay between the Host Receptor and Influenza Virus Hemagglutinin and Neuraminidase. Int. J. Mol. Sci. 18, 1541 (2017).

2. de Graaf, M. \& Fouchier, R. A. M. Role of receptor binding specificity in influenza A virus transmission and pathogenesis. EMBO J. 33, 823-841 (2014).

3. Neumann, G. \& Kawaoka, Y. Transmission of influenza A viruses. Virology 479-480, 234246 (2015).

4. Xu, R., Zhu, X., McBride, R., Nycholat, C. M., Yu, W., Paulson, J. C. \& Wilson, I. A. Functional Balance of the Hemagglutinin and Neuraminidase Activities Accompanies the Emergence of the 2009 H1N1 Influenza Pandemic. J. Virol. 86, 9221-9232 (2012).

5. Benton, D. J., Martin, S. R., Wharton, S. A. \& McCauley, J. W. Biophysical measurement of the balance of influenza $A$ hemagglutinin and neuraminidase activities. J. Biol. Chem. 290, 6516-6521 (2015).

6. Vahey, M. D. \& Fletcher, D. A. Influenza A virus surface proteins are organized to help 


\section{Chapter 2}

penetrate host mucus. elife 8, 1-24 (2019).

7. Harris, A., Cardone, G., Winkler, D. C., Heymann, J. B., Brecher, M., White, J. M. \& Steven, A. C. Influenza virus pleiomorphy characterized by cryoelectron tomography. Proc. Natl. Acad. Sci. U.S.A. 103, 19123-19127 (2006).

8. Vahey, M. D. \& Fletcher, D. A. Low-Fidelity Assembly of Influenza A Virus Promotes Escape from Host Cells. Cell 176, 281-294 (2019).

9. Sriwilaijaroen, N., Nakakita, S. I., Kondo, S., Yagi, H., Kato, K., Murata, T., Hiramatsu, H., Kawahara, T., Watanabe, Y., Kanai, Y., Ono, T., Hirabayashi, J., Matsumoto, K. \& Suzuki, $\mathrm{Y}$. N-glycan structures of human alveoli provide insight into influenza A virus infection and pathogenesis. FEBS J. 285, 1611-1634 (2018).

10. Jia, N., Byrd-Leotis, L., Matsumoto, Y., Gao, C., Wein, A. N., Lobby, J. L., Kohlmeier, J. E., Steinhauer, D. A. \& Cummings, R. D. The Human Lung Glycome Reveals Novel Glycan Ligands for Influenza A Virus. Sci. Rep. 10, 5320 (2020).

11. Long, J. S., Mistry, B., Haslam, S. M. \& Barclay, W. S. Host and viral determinants of influenza A virus species specificity. Nat. Rev. Microbiol. 17, 67-81 (2019).

12. Nagle, J. F. \& Tristram-Nagle, S. Structure of lipid bilayers. Biochim. Biophys. Acta-Rev. Biomembr. 1469, 159-195 (2000).

13. Mammen, M., Choi, S.-K. \& Whitesides, G. M. Polyvalent Interactions in Biological Systems: Implications for Design and Use of Multivalent Ligands and Inhibitors. Angew. Chem. Int. Ed. 37, 2754-2794 (1998).

14. Huskens, J., Mulder, A., Auletta, T., Nijhuis, C. A., Ludden, M. J. W. \& Reinhoudt, D. N. A model for describing the thermodynamics of multivalent host-guest interactions at interfaces. J. Am. Chem. Soc. 126, 6784-6797 (2004).

15. Huskens, J. in Multivalency 23-74 (John Wiley \& Sons, Ltd, 2017). doi:10.1002/9781119143505.ch2

16. Martinez-Veracoechea, F. J. \& Frenkel, D. Designing super selectivity in multivalent nanoparticle binding. Proc. Natl. Acad. Sci. U.S.A. 108, 10963-10968 (2011).

17. Carlson, C. B., Mowery, P., Owen, R. M., Dykhuizen, E. C. \& Kiessling, L. L. Selective Tumor Cell Targeting Using Low-Affinity, Multivalent Interactions. ACS Chem. Biol. 2, 119-127 (2007).

18. Aires, A., Cadenas, J. F., Guantes, R. \& Cortajarena, A. L. An experimental and computational framework for engineering multifunctional nanoparticles: Designing selective anticancer therapies. Nanoscale 9, 13760-13771 (2017).

19. Wang, J., Min, J., Eghtesadi, S. A., Kane, R. S. \& Chilkoti, A. Quantitative Study of the Interaction of Multivalent Ligand-Modified Nanoparticles with Breast Cancer Cells with Tunable Receptor Density. ACS Nano 14, 372-383 (2020).

20. Dubacheva, G. V., Curk, T., Mognetti, B. M., Auzély-Velty, R., Frenkel, D. \& Richter, R. P. Superselective Targeting Using Multivalent Polymers. J. Am. Chem. Soc. 136, 1722-1725 (2014).

21. Dubacheva, G. V., Curk, T., Auzély-Velty, R., Frenkel, D. \& Richter, R. P. Designing multivalent probes for tunable superselective targeting. Proc. NatI. Acad. Sci. U.S.A. 112, 5579-5584 (2015). 
22. Tito, N. B. \& Frenkel, D. Optimizing the selectivity of surface-adsorbing multivalent polymers. Macromolecules 47, 7496-7509 (2014).

23. Curk, T., Dobnikar, J. \& Frenkel, D. Optimal multivalent targeting of membranes with many distinct receptors. Proc. Natl. Acad. Sci. U.S.A. 114, 7210-7215 (2017).

24. Wang, S. \& Dormidontova, E. E. Selectivity of ligand-receptor interactions between nanoparticle and cell surfaces. Phys. Rev. Lett. 109, 1-5 (2012).

25. Curk, T. \& Tito, N. B. First-order 'hyper-selective' binding transition of multivalent particles under force. J. Phys. Condens. Matter 32, 214002 (2020).

26. Di lorio, D., Lu, Y., Meulman, J. \& Huskens, J. Recruitment of receptors at supported lipid bilayers promoted by the multivalent binding of ligand-modified unilamellar vesicles. Chem. Sci. 11, 3307-3315 (2020).

27. Albertazzi, L., Martinez-Veracoechea, F. J., Leenders, C. M. A., Voets, I. K., Frenkel, D. \& Meijer, E. W. Spatiotemporal control and superselectivity in supramolecular polymers using multivalency. Proc. Natl. Acad. Sci. U.S.A. 110, 12203-12208 (2013).

28. Verheijden, M. L. Supramolecular Binding of Vesicles, Viruses and Cells to Biomimetic Lipid Bilayers. (2018). doi:10.3990/1.9789036545501

29. Dubacheva, G. V., Curk, T., Frenkel, D. \& Richter, R. P. Multivalent Recognition at Fluid Surfaces: The Interplay of Receptor Clustering and Superselectivity. J. Am. Chem. Soc. 141, 2577-2588 (2019).

30. Mulder, A., Auletta, T., Sartori, A., Del Ciotto, S., Casnati, A., Ungaro, R., Huskens, J. \& Reinhoudt, D. N. Divalent binding of a bis(adamantyl)-functionalized calix[4]arene to beta-cyclodextrin-based hosts: An experimental and theoretical study on multivalent binding in solution and at self-assembled monolayers. J. Am. Chem. Soc. 126, 6627-6636 (2004).

31. Di lorio, D., Verheijden, M. L., van der Vries, E., Jonkheijm, P. \& Huskens, J. Weak Multivalent Binding of Influenza Hemagglutinin Nanoparticles at a SialoglycanFunctionalized Supported Lipid Bilayer. ACS Nano 13, 3413-3423 (2019).

32. Block, S. M. Kinesin Motor Mechanics: Binding, Stepping, Tracking, Gating, and Limping. Biophys. J. 92, 2986-2995 (2007).

33. Hancock, W. O. Bidirectional cargo transport: moving beyond tug of war. Nat. Rev. Mol. Cell Biol. 15, 615-628 (2014).

34. Hammer, J. A. \& Sellers, J. R. Walking to work: Roles for class v myosins as cargo transporters. Nat. Rev. Mol. Cell Biol. 13, 13-26 (2012).

35. von Delius, M. \& Leigh, D. A. Walking molecules. Chem. Soc. Rev. 40, 3656 (2011).

36. Perl, A., Gomez-Casado, A., Thompson, D., Dam, H. H., Jonkheijm, P., Reinhoudt, D. N. \& Huskens, J. Gradient-driven motion of multivalent ligand molecules along a surface functionalized with multiple receptors. Nat. Chem. 3, 317-322 (2011).

37. Satav, T., Huskens, J. \& Jonkheijm, P. Effects of Variations in Ligand Density on Cell Signaling. Small 11, 5184-5199 (2015).

38. McAuley, J. L., Gilbertson, B. P., Trifkovic, S., Brown, L. E. \& McKimm-Breschkin, J. L. Influenza Virus Neuraminidase Structure and Functions. Front. Microbiol. 10, 39 (2019). 


\section{Chapter 2}

39. Krammer, F., Smith, G. J. D., Fouchier, R. A. M., Peiris, M., Kedzierska, K., Doherty, P. C., Palese, P., Shaw, M. L., Treanor, J., Webster, R. G. \& García-Sastre, A. Influenza. Nat. Rev. Dis. Prim. 4, 3 (2018).

40. Kanagarajan, S., Tolf, C., Lundgren, A., Waldenström, J. \& Brodelius, P. E. Transient Expression of Hemagglutinin Antigen from Low Pathogenic Avian Influenza A (H7N7) in Nicotiana benthamiana. PLoS One 7, e33010 (2012).

41. Suenaga, E., Mizuno, H. \& Penmetcha, K. K. R. Monitoring influenza hemagglutinin and glycan interactions using surface plasmon resonance. Biosens. Bioelectron. 32, 195-201 (2012).

42. Guo, H., Rabouw, H., Slomp, A., Dai, M., van der Vegt, F., van Lent, J. W. M., McBride, R., Paulson, J. C., de Groot, R. J., van Kuppeveld, F. J. M., de Vries, E. \& de Haan, C. A. M. Kinetic analysis of the influenza $A$ virus HA/NA balance reveals contribution of NA to virus-receptor binding and NA-dependent rolling on receptor-containing surfaces. PLOS Pathog. 14, e1007233 (2018).

43. Tumpey, T. M. Characterization of the Reconstructed 1918 Spanish Influenza Pandemic Virus. Science 310, 77-80 (2005).

44. Matrosovich, M., Tuzikov, A., Bovin, N., Gambaryan, A., Klimov, A., Castrucci, M. R., Donatelli, I. \& Kawaoka, Y. Early Alterations of the Receptor-Binding Properties of H1, $\mathrm{H} 2$, and H3 Avian Influenza Virus Hemagglutinins after Their Introduction into Mammals. J. Virol. 74, 8502-8512 (2000).

45. Yen, H.-L., Liang, C.-H., Wu, C.-Y., Forrest, H. L., Ferguson, A., Choy, K.-T., Jones, J., Wong, D. D.-Y., Cheung, P. P.-H., Hsu, C.-H., Li, O. T., Yuen, K. M., Chan, R. W. Y., Poon, L. L. M. M., Chan, M. C. W. W., Nicholls, J. M., Krauss, S., Wong, C.-H., Guan, Y., Webster, R. G., Webby, R. J. \& Peiris, M. Hemagglutinin-neuraminidase balance confers respiratorydroplet transmissibility of the pandemic H1N1 influenza virus in ferrets. Proc. Natl. Acad. Sci. U.S.A. 108, 14264-14269 (2011).

46. Xiong, X., Coombs, P. J., Martin, S. R., Liu, J., Xiao, H., McCauley, J. W., Locher, K., Walker, P. A., Collins, P. J., Kawaoka, Y., Skehel, J. J. \& Gamblin, S. J. Receptor binding by a ferrettransmissible H5 avian influenza virus. Nature 497, 392-396 (2013).

47. Vachieri, S. G., Xiong, X., Collins, P. J., Walker, P. a, Martin, S. R., Haire, L. F., Zhang, Y., McCauley, J. W., Gamblin, S. J. \& Skehel, J. J. Receptor binding by H10 influenza viruses. Nature 511, 475-477 (2014).

48. Hirst, G. K. The Agglutination of Red Cells by Allantoic Fluid of Chick Embryos Infected with Influenza Virus. Science 94, 22-23 (1941).

49. Killian, M. L. in Methods Mol. Biol. 1161, 3-9 (2014).

50. Rogers, G. N., Pritchett, T. J., Lane, J. L. \& Paulson, J. C. Differential sensitivity of human, avian, and equine influenza a viruses to a glycoprotein inhibitor of infection: Selection of receptor specific variants. Virology 131, 394-408 (1983).

51. Ji, Y., White, Y. J., Hadden, J. A., Grant, O. C. \& Woods, R. J. New insights into influenza A specificity: an evolution of paradigms. Curr. Opin. Struct. Biol. 44, 219-231 (2017).

52. Lin, Y. P., Xiong, X., Wharton, S. A., Martin, S. R., Coombs, P. J., Vachieri, S. G., Christodoulou, E., Walker, P. A., Liu, J., Skehel, J. J., Gamblin, S. J., Hay, A. J., Daniels, R. 
S. \& McCauley, J. W. Evolution of the receptor binding properties of the influenza A(H3N2) hemagglutinin. Proc. Natl. Acad. Sci. U.S.A. 109, 21474-21479 (2012).

53. Stevens, J., Blixt, O., Tumpey, T. M., Taubenberger, J. K., Paulson, J. C. \& Wilson, I. A. Structure and Receptor Specificity of the Hemagglutinin from an H5N1 Influenza Virus. Science 312, 404-410 (2006).

54. Blixt, O., Head, S., Mondala, T., Scanlan, C., Huflejt, M. E., Alvarez, R., Bryan, M. C., Fazio, F., Calarese, D., Stevens, J., Razi, N., Stevens, D. J., Skehel, J. J., van Die, I., Burton, D. R., Wilson, I. a, Cummings, R., Bovin, N., Wong, C.-H. \& Paulson, J. C. Printed covalent glycan array for ligand profiling of diverse glycan binding proteins. Proc. Natl. Acad. Sci. U.S.A. 101, 17033-17038 (2004).

55. Liao, H. Y., Hsu, C. H., Wang, S. C., Liang, C. H., Yen, H. Y., Su, C. Y., Chen, C. H., Jan, J. T., Ren, C. T., Chen, C. H., Cheng, T. J. R., Wu, C. Y. \& Wong, C. H. Differential receptor binding affinities of influenza hemagglutinins on glycan arrays. J. Am. Chem. Soc. 132, 1484914856 (2010).

56. McQuillan, A. M., Byrd-Leotis, L., Heimburg-Molinaro, J. \& Cummings, R. D. Natural and Synthetic Sialylated Glycan Microarrays and Their Applications. Front. Mol. Biosci. 6, 88 (2019).

57. Smith, D. F. \& Cummings, R. D. Investigating virus-glycan interactions using glycan microarrays. Curr. Opin. Virol. 7, 79-87 (2014).

58. Stevens, J., Blixt, O., Glaser, L., Taubenberger, J. K., Palese, P., Paulson, J. C. \& Wilson, I. A. Glycan microarray analysis of the hemagglutinins from modern and pandemic influenza viruses reveals different receptor specificities. J. Mol. Biol. 355, 1143-1155 (2006).

59. Stevens, J., Blixt, O., Chen, L. M., Donis, R. O., Paulson, J. C. \& Wilson, I. A. Recent Avian H5N1 Viruses Exhibit Increased Propensity for Acquiring Human Receptor Specificity. J. Mol. Biol. 381, 1382-1394 (2008).

60. Li, Z. \& Chai, W. Mucin O-glycan microarrays. Curr. Opin. Struct. Biol. 56, 187-197 (2019).

61. Song, X., Lasanajak, Y., Xia, B., Heimburg-Molinaro, J., Rhea, J. M., Ju, H., Zhao, C., Molinaro, R. J., Cummings, R. D. \& Smith, D. F. Shotgun glycomics: a microarray strategy for functional glycomics. Nat. Methods 8, 85-90 (2011).

62. Byrd-Leotis, L., Jia, N., Dutta, S., Trost, J. F., Gao, C., Cummings, S. F., Braulke, T., MüllerLoennies, S., Heimburg-Molinaro, J., Steinhauer, D. A. \& Cummings, R. D. Influenza binds phosphorylated glycans from human lung. Sci. Adv. 5, 1-10 (2019).

63. Huang, M. L., Cohen, M., Fisher, C. J., Schooley, R. T., Gagneux, P. \& Godula, K. Determination of receptor specificities for whole influenza viruses using multivalent glycan arrays. Chem. Commun. 51, 5326-5329 (2015).

64. Di lorio, D. \& Huskens, J. Surface Modification with Control over Ligand Density for the Study of Multivalent Biological Systems. ChemistryOpen 9, 53-66 (2020).

65. Di lorio, D. Designer Surfaces for the Quantification of Multivalent Biological Interactions. (2019). doi:10.3990/1.9789036548199

66. Van Weerd, J., Krabbenborg, S. O., Eijkel, J., Karperien, M., Huskens, J. \& Jonkheijm, P. On-Chip Electrophoresis in Supported Lipid Bilayer Membranes Achieved Using Low 


\section{Chapter 2}

Potentials. J. Am. Chem. Soc. 136, 100-103 (2014).

67. Krabbenborg, S. O., van Weerd, J., Karperien, M., Jonkheijm, P. \& Huskens, J. Locked-in Biomimetic Surface Gradients that are Tunable in Size, Density and Functionalization. ChemPhysChem 15, 3460-3465 (2014).

68. Reiter-Scherer, V., Cuellar-Camacho, J. L., Bhatia, S., Haag, R., Herrmann, A., Lauster, D. \& Rabe, J. P. Force Spectroscopy Shows Dynamic Binding of Influenza Hemagglutinin and Neuraminidase to Sialic Acid. Biophys. J. 116, 1037-1048 (2019).

69. Cuellar-Camacho, J. L., Bhatia, S., Reiter-Scherer, V., Lauster, D., Liese, S., Rabe, J. P., Herrmann, A. \& Haag, R. Quantification of Multivalent Interactions between Sialic Acid and Influenza A Virus Spike Proteins by Single-Molecule Force Spectroscopy. J. Am. Chem. Soc. 142, 12181-12192 (2020).

70. Xu, R., Zhu, X., McBride, R., Nycholat, C. M., Yu, W., Paulson, J. C. \& Wilson, I. A. Functional balance of the hemagglutinin and neuraminidase activities accompanies the emergence of the 2009 H1N1 influenza pandemic. J. Virol. 86, 9221-32 (2012).

71. Gaymard, A., Le Briand, N., Frobert, E., Lina, B. \& Escuret, V. Functional balance between neuraminidase and haemagglutinin in influenza viruses. Clin. Microbiol. Infect. 22, 975983 (2016).

72. Gambaryan, A. S. \& Matrosovich, M. N. What adaptive changes in hemagglutinin and neuraminidase are necessary for emergence of pandemic influenza virus from its avian precursor? Biochem. 80, 872-880 (2015).

73. Wagner, R., Matrosovich, M. \& Klenk, H.-D. Functional balance between haemagglutinin and neuraminidase in influenza virus infections. Rev. Med. Virol. 12, 159-166 (2002).

74. Behera, A. K., Basu, S. \& Cherian, S. S. Molecular mechanism of the enhanced viral fitness contributed by secondary mutations in the hemagglutinin protein of oseltamivir resistant H1N1 influenza viruses: Modeling studies of antibody and receptor binding. Gene 557, 19-27 (2015).

75. Ward, M. J., Lycett, S. J., Avila, D., Bollback, J. P. \& Leigh Brown, A. J. Evolutionary interactions between haemagglutinin and neuraminidase in avian influenza. BMC Evol. Biol. 13, 222 (2013).

76. Diederich, S., Berhane, Y., Embury-Hyatt, C., Hisanaga, T., Handel, K., Cottam-Birt, C., Ranadheera, C., Kobasa, D. \& Pasick, J. Hemagglutinin-Neuraminidase Balance Influences the Virulence Phenotype of a Recombinant H5N3 Influenza A Virus Possessing a Polybasic HA 0 Cleavage Site. J. Virol. 89, 10724-10734 (2015).

77. Gulati, U., Wu, W., Gulati, S., Kumari, K., Waner, J. L. \& Air, G. M. Mismatched hemagglutinin and neuraminidase specificities in recent human H3N2 influenza viruses. Virology 339, 12-20 (2005).

78. Baigent, S. J. \& McCauley, J. W. Glycosylation of haemagglutinin and stalk-length of neuraminidase combine to regulate the growth of avian influenza viruses in tissue culture. Virus Res. 79, 177-185 (2001).

79. Gen, F., Yamada, S., Kato, K., Akashi, H., Kawaoka, Y. \& Horimoto, T. Attenuation of an influenza $A$ virus due to alteration of its hemagglutinin-neuraminidase functional balance in mice. Arch. Virol. 158, 1003-1011 (2013). 
80. Chen, Q., Huang, S., Chen, J., Zhang, S. \& Chen, Z. NA Proteins of Influenza A Viruses H1N1/2009, H5N1, and H9N2 Show Differential Effects on Infection Initiation, Virus Release, and Cell-Cell Fusion. PLoS One 8, e54334 (2013).

81. Button, B., Cai, L.-H., Ehre, C., Kesimer, M., Hill, D. B., Sheehan, J. K., Boucher, R. C. \& Rubinstein, M. A Periciliary Brush Promotes the Lung Health by Separating the Mucus Layer from Airway Epithelia. Science 337, 937-941 (2012).

82. Kesimer, M., Ehre, C., Burns, K. A., Davis, C. W., Sheehan, J. K. \& Pickles, R. J. Molecular organization of the mucins and glycocalyx underlying mucus transport over mucosal surfaces of the airways. Mucosal Immunol. 6, 379-392 (2013).

83. Benton, D. J., Wharton, S. A., Martin, S. R. \& McCauley, J. W. Role of Neuraminidase in Influenza A(H7N9) Virus Receptor Binding. J. Virol. 91, 1-10 (2017).

84. Dai, M., McBride, R., Dortmans, J. C. F. M., Peng, W., Bakkers, M. J. G., de Groot, R. J., van Kuppeveld, F. J. M., Paulson, J. C., de Vries, E. \& de Haan, C. A. M. Mutation of the Second Sialic Acid-Binding Site, Resulting in Reduced Neuraminidase Activity, Preceded the Emergence of H7N9 Influenza A Virus. J. Virol. 91, e00049-17 (2017).

85. Du, W., Dai, M., Li, Z., Boons, G.-J., Peeters, B., van Kuppeveld, F. J. M., de Vries, E. \& de Haan, C. A. M. Substrate Binding by the Second Sialic Acid-Binding Site of Influenza A Virus N1 Neuraminidase Contributes to Enzymatic Activity. J. Virol. 92, JVI.01243-18 (2018).

86. Bansil, R. \& Turner, B. S. The biology of mucus: Composition, synthesis and organization. Adv. Drug Deliv. Rev. 124, 3-15 (2018).

87. Taherali, F., Varum, F. \& Basit, A. W. A slippery slope: On the origin, role and physiology of mucus. Adv. Drug Deliv. Rev. 124, 16-33 (2018).

88. Schuster, B. S., Suk, J. S., Woodworth, G. F. \& Hanes, J. Nanoparticle diffusion in respiratory mucus from humans without lung disease. Biomaterials 34, 3439-3446 (2013).

89. Gagneux, P., Cheriyan, M., Hurtado-Ziola, N., van der Linden, E. C. M. B., Anderson, D., McClure, H., Varki, A. \& Varki, N. M. Human-specific Regulation of a2-6-linked Sialic Acids. J. Biol. Chem. 278, 48245-48250 (2003).

90. Couceiro, J. N. S. S., Paulson, J. C. \& Baum, L. G. Influenza virus strains selectively recognize sialyloligosaccharides on human respiratory epithelium; the role of the host cell in selection of hemagglutinin receptor specificity. Virus Res. 29, 155-165 (1993).

91. Sakai, T., Nishimura, S. I., Naito, T. \& Saito, M. Influenza A virus hemagglutinin and neuraminidase act as novel motile machinery. Sci. Rep. 7, 45043 (2017).

92. Seladi-Schulman, J., Steel, J. \& Lowen, A. C. Spherical Influenza Viruses Have a Fitness Advantage in Embryonated Eggs, while Filament-Producing Strains Are Selected In Vivo. J. Virol. 87, 13343-13353 (2013).

93. Hamming, P. H., Overeem, N. J. \& Huskens, J. Influenza as a molecular walker. Chem. Sci. 11, 27-36 (2020).

94. Dadonaite, B., Vijayakrishnan, S., Fodor, E., Bhella, D. \& Hutchinson, E. C. Filamentous influenza viruses. J. Gen. Virol. 97, 1755-1764 (2016).

95. de Vries, E., Du, W., Guo, H. \& de Haan, C. A. M. Influenza A Virus Hemagglutinin- 


\section{Chapter 2}

Neuraminidase-Receptor Balance: Preserving Virus Motility. Trends Microbiol. 28, 5767 (2020).

96. Shinya, K., Ebina, M., Yamada, S., Ono, M., Kasai, N. \& Kawaoka, Y. Influenza virus receptors in the human airway. Nature 440, 435-436 (2006).

97. Van Riel, D., Munster, V. J., de Wit, E., Rimmelzwaan, G. F., Fouchier, R. A. M., Osterhaus, A. D. M. E. \& Kuiken, T. H5N1 Virus Attachment to Lower Respiratory Tract. Science 312, 399-399 (2006).

98. Matrosovich, M. N., Matrosovich, T. Y., Gray, T., Roberts, N. A. \& Klenk, H.-D. Human and avian influenza viruses target different cell types in cultures of human airway epithelium. Proc. Natl. Acad. Sci. U.S.A. 101, 4620-4624 (2004).

99. Shelton, H., Ayora-Talavera, G., Ren, J., Loureiro, S., Pickles, R. J., Barclay, W. S. \& Jones, I. M. Receptor Binding Profiles of Avian Influenza Virus Hemagglutinin Subtypes on Human Cells as a Predictor of Pandemic Potential. J. Virol. 85, 1875-1880 (2011).

100. Richard, M., van den Brand, J. M. A., Bestebroer, T. M., Lexmond, P., de Meulder, D., Fouchier, R. A. M., Lowen, A. C. \& Herfst, S. Influenza A viruses are transmitted via the air from the nasal respiratory epithelium of ferrets. Nat. Commun. 11, 766 (2020).

101. Davis, A. S., Chertow, D. S., Kindrachuk, J., Qi, L., Schwartzman, L. M., Suzich, J., Alsaaty, S., Logun, C., Shelhamer, J. H. \& Taubenberger, J. K. 1918 Influenza receptor binding domain variants bind and replicate in primary human airway cells regardless of receptor specificity. Virology 493, 238-246 (2016).

102. Block, S., Zhdanov, V. P. \& Höök, F. Quantification of multivalent interactions by tracking single biological nanoparticle mobility on a lipid membrane. Nano Lett. 16, 4382-4390 (2016).

103. Müller, M., Lauster, D., Wildenauer, H. H. K., Herrmann, A. \& Block, S. Mobility-Based Quantification of Multivalent Virus-Receptor Interactions: New Insights Into Influenza A Virus Binding Mode. Nano Lett. 19, 1875-1882 (2019).

104. Rust, M. J., Lakadamyali, M., Zhang, F. \& Zhuang, X. Assembly of endocytic machinery around individual influenza viruses during viral entry. Nat. Struct. Mol. Biol. 11, 567-573 (2004).

105. Rossman, J. S., Leser, G. P. \& Lamb, R. A. Filamentous Influenza Virus Enters Cells via Macropinocytosis. J. Virol. 86, 10950-10960 (2012).

106. Chen, C. \& Zhuang, X. Epsin 1 is a cargo-specific adaptor for the clathrin-mediated endocytosis of the influenza virus. Proc. Natl. Acad. Sci. U.S.A. 105, 11790-11795 (2008).

107. Chan, C. M., Chu, H., Zhang, A. J., Leung, L. H., Sze, K. H., Kao, R. Y. T., Chik, K. K. H., To, K. K. W., Chan, J. F. W., Chen, H., Jin, D. Y., Liu, L. \& Yuen, K. Y. Hemagglutinin of influenza A virus binds specifically to cell surface nucleolin and plays a role in virus internalization. Virology 494, 78-88 (2016).

108. de Vries, E., Tscherne, D. M., Wienholts, M. J., Cobos-Jiménez, V., Scholte, F., GarcíaSastre, A., Rottier, P. J. M. \& de Haan, C. A. M. Dissection of the Influenza A Virus Endocytic Routes Reveals Macropinocytosis as an Alternative Entry Pathway. PLoS Pathog. 7, e1001329 (2011).

109. Ramakrishnan, N., Tourdot, R. W., Eckmann, D. M., Ayyaswamy, P. S., Muzykantov, V. R. 
\& Radhakrishnan, R. Biophysically inspired model for functionalized nanocarrier adhesion to cell surface: roles of protein expression and mechanical factors. R. Soc. Open Sci. 3, 160260 (2016).

110. Grove, J. \& Marsh, M. The cell biology of receptor-mediated virus entry. J. Cell Biol. 195, 1071-1082 (2011).

111. Ohuchi, M., Asaoka, N., Sakai, T. \& Ohuchi, R. Roles of neuraminidase in the initial stage of influenza virus infection. Microbes Infect. 8, 1287-1293 (2006).

112. Hoja-Łukowicz, D., Kedracka-Krok, S., Duda, W. \& Lityńska, A. The lectin-binding pattern of nucleolin and its interaction with endogenous galectin-3. Cell. Mol. Biol. Lett. 19, 461482 (2014).

113. Eierhoff, T., Hrincius, E. R., Rescher, U., Ludwig, S. \& Ehrhardt, C. The Epidermal Growth Factor Receptor (EGFR) Promotes Uptake of Influenza A Viruses (IAV) into Host Cells. PLoS Pathog. 6, e1001099 (2010).

114. Fujioka, Y., Tsuda, M., Nanbo, A., Hattori, T., Sasaki, J., Sasaki, T., Miyazaki, T. \& Ohba, Y. A Ca2+-dependent signalling circuit regulates influenza $A$ virus internalization and infection. Nat. Commun. 4, 2763 (2013).

115. Fujioka, Y., Nishide, S., Ose, T., Suzuki, T., Kato, I., Fukuhara, H., Fujioka, M., Horiuchi, K., Satoh, A. O., Nepal, P., Kashiwagi, S., Wang, J., Horiguchi, M., Sato, Y., Paudel, S., Nanbo, A., Miyazaki, T., Hasegawa, H., Maenaka, K. \& Ohba, Y. A Sialylated Voltage-Dependent Ca 2+ Channel Binds Hemagglutinin and Mediates Influenza A Virus Entry into Mammalian Cells. Cell Host Microbe 23, 809-818 (2018).

116. Dixon, R. E., Moreno, C. M., Yuan, C., Opitz-Araya, X., Binder, M. D., Navedo, M. F. \& Santana, L. F. Graded Ca2+/calmodulin-dependent coupling of voltage-gated CaV1.2 channels. eLife 4, 1-21 (2015).

117. Chu, V. C. \& Whittaker, G. R. Influenza virus entry and infection require host cell N-linked glycoprotein. Proc. Natl. Acad. Sci. U.S.A. 101, 18153-18158 (2004).

118. de Vries, E., de Vries, R. P., Wienholts, M. J., Floris, C. E., Jacobs, M.-S., van den Heuvel, A., Rottier, P. J. M. \& de Haan, C. A. M. Influenza A virus entry into cells lacking sialylated $\mathrm{N}$-glycans. Proc. Natl. Acad. Sci. U.S.A. 109, 7457-7462 (2012).

119. Goronzy, I. N., Rawle, R. J., Boxer, S. G. \& Kasson, P. M. Cholesterol enhances influenza binding avidity by controlling nanoscale receptor clustering. Chem. Sci. 9, 2340-2347 (2018).

120. Matrosovich, M., Suzuki, T., Hirabayashi, Y., Garten, W., Webster, R. G. \& Klenk, H. D. Gangliosides are not essential for influenza virus infection. Glycoconj. J. 23, 107-113 (2006).

121. Yang, J., Liu, S., Du, L. \& Jiang, S. A new role of neuraminidase (NA) in the influenza virus life cycle: implication for developing NA inhibitors with novel mechanism of action. Rev. Med. Virol. 26, 242-250 (2016).

122. Savage, N. The push for better flu therapies. Nature 573, S54-S55 (2019).

123. Pizzorno, A., Terrier, O., Nicolas de Lamballerie, C., Julien, T., Padey, B., Traversier, A., Roche, M., Hamelin, M.-E., Rhéaume, C., Croze, S., Escuret, V., Poissy, J., Lina, B., LegrasLachuer, C., Textoris, J., Boivin, G. \& Rosa-Calatrava, M. Repurposing of Drugs as Novel 


\section{Chapter 2}

Influenza Inhibitors From Clinical Gene Expression Infection Signatures. Front. Immunol. 10, 60 (2019).

124. Bhatia, S., Camacho, L. C. \& Haag, R. Pathogen Inhibition by Multivalent Ligand Architectures. J. Am. Chem. Soc. 138, 8654-8666 (2016).

125. Lauster, D., Klenk, S., Ludwig, K., Nojoumi, S., Behren, S., Adam, L., Stadtmüller, M., Saenger, S., Zimmler, S., Hönzke, K., Yao, L., Hoffmann, U., Bardua, M., Hamann, A., Witzenrath, M., Sander, L. E., Wolff, T., Hocke, A. C., Hippenstiel, S., De Carlo, S., Neudecker, J., Osterrieder, K., Budisa, N., Netz, R. R., Böttcher, C., Liese, S., Herrmann, A. \& Hackenberger, C. P. R. Phage capsid nanoparticles with defined ligand arrangement block influenza virus entry. Nat. Nanotechnol. 15, 373-379 (2020). 
A dynamic, supramolecular view on the multivalent virus-host cell interaction 


\section{Chapter 3}

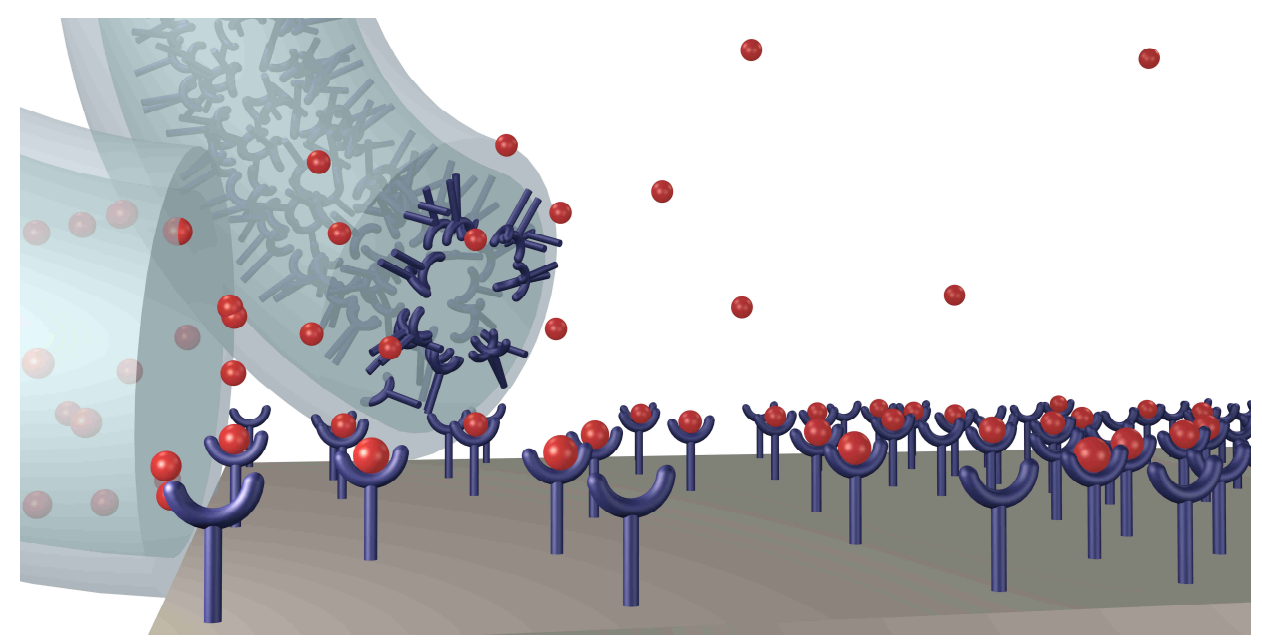

\section{Highlights}

- A microfluidic sensor with receptors on a supported lipid bilayer was studied with fluorescence microscopy and finite element simulations

- Analyte molecules arrive late in the sensing area because receptors bind nonspecifically to the tubing

- With a secondary inlet for the assembly of the supported lipid bilayer and the immobilization of receptors, the analyte is no longer delayed

- Proteins and nanoparticles are also depleted by nonspecifically bound receptors, but not delayed 


\section{Time-dependent binding of molecules and nanoparticles at receptor-modified supported lipid bilayer gradients in a microfluidic device}

Microfluidic devices are widely used for the sensing of small quantities of analytes. In these applications, the measurement can be easily perturbed by loss of analyte due to binding of the analyte outside the sensing area. We studied the binding of small molecules and nanoparticles up to $400 \mathrm{~nm}$ in a state-of-the-art sensing platform receptor gradients on supported lipid bilayers (SLBs) - in a microfluidic device over time. Biotin-streptavidin was used as the model interaction motif for specific binding and a biotin-modified dye, which can bind to the streptavidin on the SLB, as a small-molecule model analyte. We used finite element simulations to show that the time-dependent binding of analytes in the sensing area depends strongly on the extent of the nonspecific binding of the vesicles, used in a preceding step to make the SLB platform, outside of the sensing area (e.g., in the tubing). Proteins and nanoparticles were only partially depleted by nonspecifically adsorbed lipids and no delayed onset of binding was observed, which was attributed to their lower diffusion coefficients. As a practical solution, a flow cell with two inlets was used to avoid the presence of nonspecifically adsorbed receptors in the sample inlet, which allowed us to decouple the formation of the sensor layer on the surface from the subsequent sensing event. We found that in the absence of lipids adsorbed to the tubing, the nonspecific binding of dye molecules was negligible.

Part of this chapter has been published in:

Overeem, N. J., Hamming, P. H., Huskens, J., ChemistrySelect 2020, 5, 1-8. 


\subsection{Introduction}

Microfluidic devices are widely used for sensing, both in point-of-care devices and in research applications. ${ }^{1,2}$ The need for only small volumes is a key advantage for studies that use biological samples, where the species of interest is usually available in limited quantities only. ${ }^{3,4}$ Many biosensors use a method in which a sample is passed over a surface bearing receptors to which the species of interest binds selectively. ${ }^{5,6}$ In such methods, the species of interest can be present in low concentrations, especially when such species are multivalent or strongly binding, such as cells, bacteria, viruses, protein assemblies and nucleic acids. ${ }^{7-10}$

Receptor density surface gradients in a microfluidic device offer a novel and state-of-the-art platform for the study of such supramolecular interactions. ${ }^{11,12}$ Our group has developed a microfluidic flow cell to create supported lipid bilayers (SLBs) on the surface of a chip and form an electrophoretic gradient within the SLBs. ${ }^{13}$ Using SLBs of saturated lipids, gradients have been formed at an elevated temperature and locked-in by cooling, yielding stable surface gradients that allow a variety of covalent and noncovalent modifications. ${ }^{14}$ This technique was used to create density gradients of thiolated mannose on maleimide-modified lipids to study the binding of uropathogenic E. coli. ${ }^{12}$ The continuous variation of receptor density over the length of an SLB allows the performance of a receptor density titration in a single fluorescence micrograph.

It is especially instructive to apply this method to develop a sensor for viruses. Viruses behave as multivalent particles with binding properties adapted to circumvent the immune system and find the required target (see also Chapter 2). ${ }^{15-17}$ To quantitatively assess the binding of particles to receptors on the surface, it is important that high enough numbers of particles are bound and that the binding is not disrupted by the fluid flow.

Here it is shown that the loss of analyte outside the sensing area in a microfluidic device that has receptors displayed on an SLB, is governed by nonspecific interactions of the SLB-forming lipids with the tubing and by the diffusion constant of the analyte, for both small molecules and nanoparticles in sub-micromolar concentrations. We studied the time-dependent binding of dyes and particles with fluorescence microscopy to discover how receptor 
density, ligand concentration and flow rate affect the time required until half of the saturation coverage is reached $\left(t_{1 / 2}\right)$. We used a finite element model to simulate this time-dependent binding and found that for low concentrations of analyte, the major contribution to $t_{1 / 2}$ is binding outside of the sensing area (i.e., at the tubing). To prevent the analyte from flowing along nonspecifically bound receptors in the tubing, we introduced a secondary inlet to decouple the SLB formation process from the introduction of analytes. We compared the adsorption profiles of both single and double inlet devices to assess the effect on the analyte adsorption outside the sensing area.

\subsection{Results and discussion}

\subsubsection{Gradient formation in SLBS}

To study the binding of small molecules and nanoparticles to a surface with receptors in a flow cell, we used receptor gradients on SLBs (Figure 3.1). The gradients allowed us to distinguish the binding of fluorescently labeled model analytes (molecules and particles) from background fluorescence, without the need for total internal reflection fluorescence microscopy (TIRF) or confocal microscopy. We used a flow cell with a straight, rectangular microchannel on a glass chip, on which three positions between interdigitated gold electrodes for supported lipid bilayers (SLBs) are subdivided into $100 \mu \mathrm{m}$ corrals by chromium lines (Figures 3.1a and 3.2). 


\section{Chapter 3}

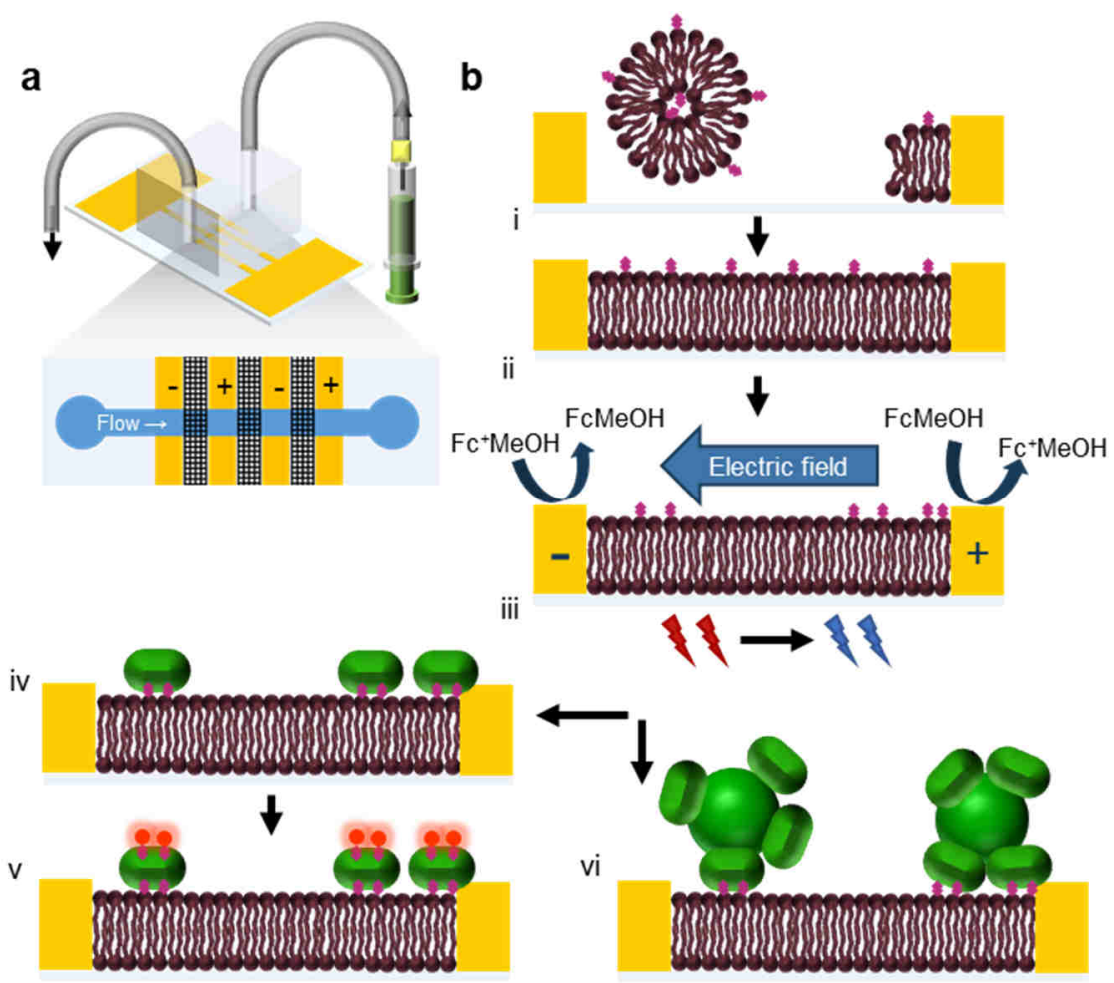

Figure 3.1| Schematic representation of the method used to form receptor gradients and study ligand binding. a) A flow cell, fed by a syringe pump, with a PDMS microchannel on a glass chip, in which there are three positions between interdigitated gold electrodes for supported lipid bilayers (SLBs) that are subdivided into corrals by chromium lines. b) Process to create receptor gradients on SLBs: (i) on glass, an SLB is formed by vesicle rupture at a temperature above the $T_{\mathrm{m}}$ of the lipids. (ii) A potential is applied to form an electrophoretic gradient of biotin-DOPE lipids in the SLBs. Ferrocene methanol is used as sacrificial agent to prevent water splitting. (iii) The gradients are locked in by rapidly cooling the flow cell to room temperature. (iv) For small molecule binding studies, the gradients are functionalized with fluorescently labelled streptavidin (SAv) that can act as receptor. (v) Biotinylated fluorescent dyes are passed through the microchannel and bind to the surface as model analyte. The binding is monitored with fluorescence microscopy. (iv) For particle binding studies, SAv-coated fluorescent nanoparticles act as analyte, binding directly to the biotin-DOPE lipids. 

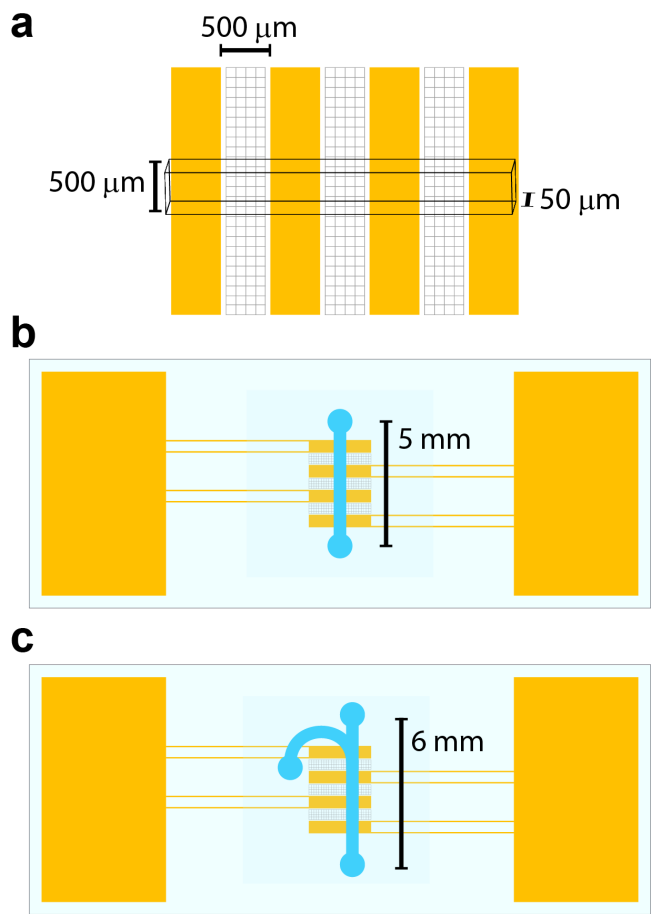

Figure 3.2| Schematic representation of the gradient chips and microchannels. a) Interdigitated electrodes with corrals subdivided by chromium and cross-section dimensions of the microchannels. b) Shape and length of the straight microchannel. c) Shape and length of the microchannel with secondary inlet. With a secondary inlet, the microchannel is moved to the right, so that all in- and outlets have enough PDMS around it.

We created SLBs of the zwitterionic lipid 1-myristoyl-2-palmitoyl-sn-glycero-3phosphocholine (MPPC) and the negatively charged, biotinylated lipid 1,2dioleoyl-sn-glycero-3-phosphoethanolamine (biotin-DOPE) (Figure 3.1b). Electrophoretic gradients in biotin density were formed by applying a potential over the SLBs at $50^{\circ} \mathrm{C}$. The elevated temperature results in a fluid state SLB which allows the negatively charged lipids to migrate in the direction of the cathode. Afterwards, the flow cells were cooled to room temperature, resulting in a gel state SLB which locks-in the gradients. The SLBs were then functionalized using streptavidin (SAv). SAv labelled with fluorescent CF350 or Alexa Fluor 488 (AF488) was passed through the flow cell using a syringe pump at $10 \mu \mathrm{L} / \mathrm{min}$ in a concentration of $20 \mu \mathrm{g} / \mathrm{mL}(333 \mathrm{nM})$. When we recorded 
fluorescence micrographs of the surface, the fluorescence was found to reach its maximum intensity after approx. 8 min (Figure 3.3). We passed SAv through the flow cells for $25 \mathrm{~min}$ to ensure saturation of the SLBs and rinsed with phosphate-buffered saline (PBS) to remove unbound SAv.

\subsubsection{Time-dependent binding of small molecules}

We used a fluorescent ligand, ATTO 565-biotin, to study how receptor density and analyte concentration affect the saturation half-life, $t_{1 / 2}$. After successful formation of the SAv-coated gradients, ATTO 565-biotin was flowed through the flow cell and fluorescence micrographs were recorded at regular intervals. The fluorescence micrographs were processed by a custom written MATLAB script to extract the average fluorescence intensities of ATTO 565-biotin on the SLBs and remove the background fluorescence that was visible outside the microchannel.

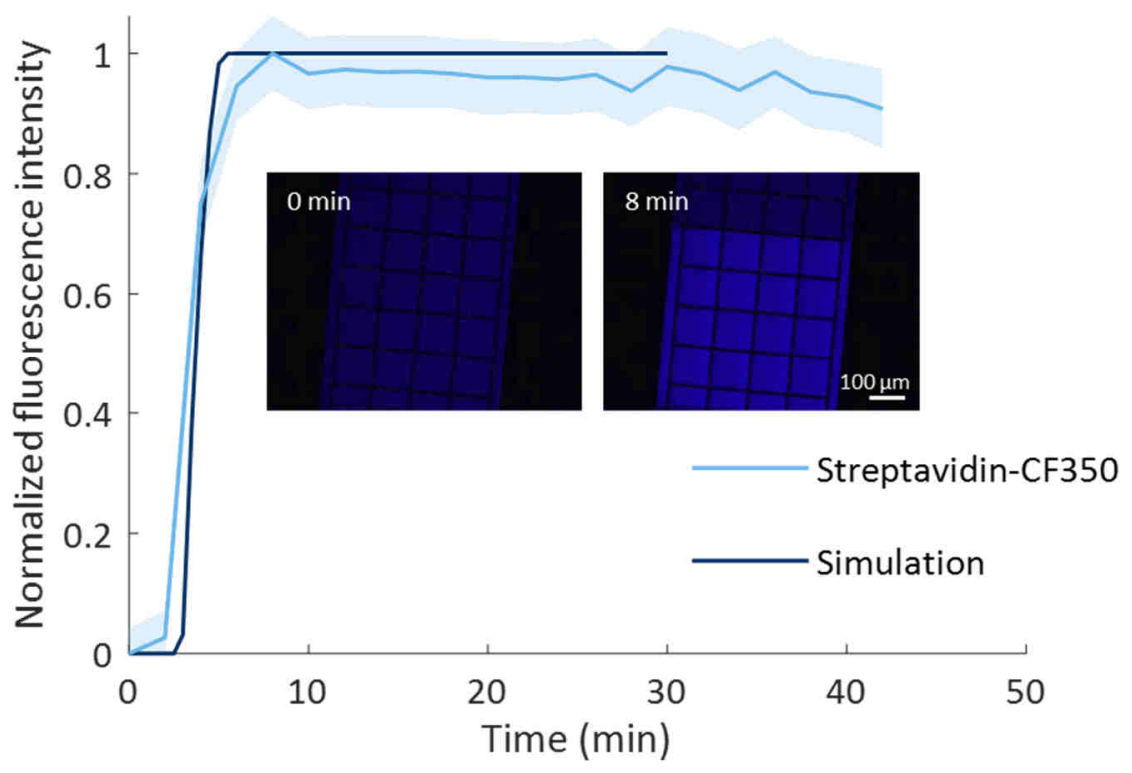

Figure 3.3| Binding profile of SAv-CF350 to biotinylated SLBs. Experimental and simulated profiles are shown. Inset shows fluorescent micrographs before adding SAvCF350 and after $8 \mathrm{~min}$, when the surface is saturated. 
Figure 3.4 shows fluorescence micrographs of the inlet and corrals of a microchannel with SAv-AF488-modified SLBs before and after incubation with ATTO 565-biotin. The intense green fluorescence at the inlet in Figure 3.4a indicates that SAV-AF488 has not only bound to the SLB on the glass, but also to the Tygon tubing and polydimethylsiloxane (PDMS) channel walls. The higher intensity of red fluorescence at the inlet than at the corrals must arise from background fluorescence. During the analyte binding phase (Figure 3.4b), the red intensity increases due to the presence of ATTO 565-biotin and the green fluorescence intensity decreases, suggesting that some SAv is released either by the prolonged rinsing or by the presence of competing biotin. After washing with PBS (Figure 3.4c), the ratio of red to green fluorescence intensities is similar in the tubing and at the corrals, indicating that ATTO 565-biotin binds not only on the corrals, but also to the tubing.

In Figure 3.4d, the increasing fluorescence intensity shows how the dye binds over time. Passing through only $30-40 \mu \mathrm{L}$ of tubing at a flow rate of $10 \mu \mathrm{L} / \mathrm{min}$, the dye was expected to reach the microchannel at 3-4 min but typically it took around $16 \mathrm{~min}$ with some samples taking up to $40 \mathrm{~min}$ under the same experimental conditions. Further binding curves are shown in Figure 3.5. This delayed transport to the corrals suggests that much of the dye is depleted from the solution in the tubing before it reaches the microchannel. 


\section{Chapter 3}

a

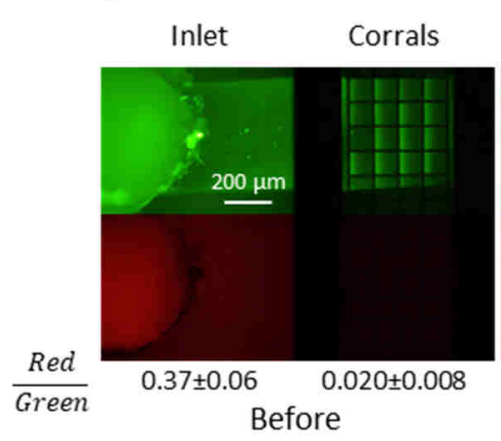

C

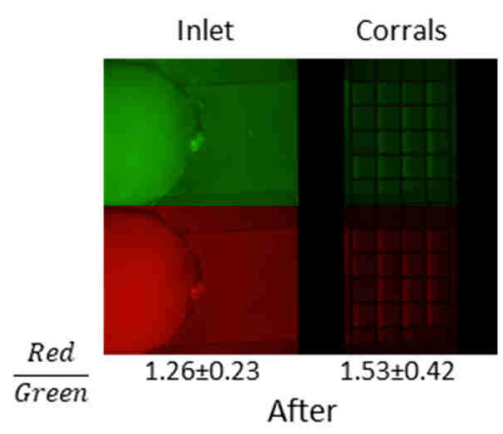

b

Inlet Corrals

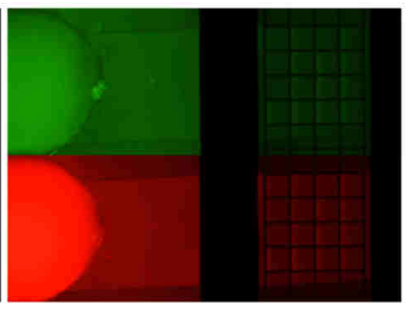

During

d

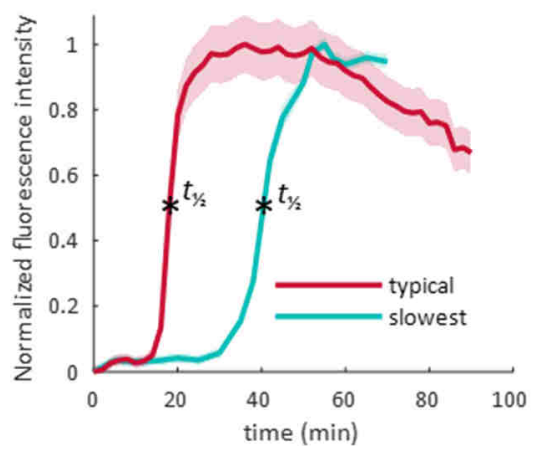

Figure 3.4 | Binding of ATTO 565-biotin (red) to SAv-AF488 (green) in the tubing and corrals followed over time with fluorescence microscopy. b) Fluorescence micrographs of SAv-AF488 (green) at the inlet and corrals of the flow cell before ATTO 565-biotin (red) is added. The ratio of red over green fluorescence intensities on the surface in the center of the inlet and on the corrals is shown below the micrographs. b) Fluorescence micrographs of the addition of ATTO 565-biotin. c) Fluorescence micrographs after the ATTO 565-biotin solution is washed from the microchannel. d) Time-dependent binding profiles of ATTO 565-biotin to streptavidin-coated SLBs, with $0.5 \%$ biotin in the SLBs and $40 \mathrm{nM}$ ATTO 565-biotin in the solution. The fluorescence intensity is averaged over all corrals inside the microchannel and the background intensity was determined in a corral outside the microchannel before normalization. Both a typical and the slowest curve are shown. Shaded areas represent the standard errors between corrals. In both curves, $t_{1} / 2$ is indicated with an asterisk. 

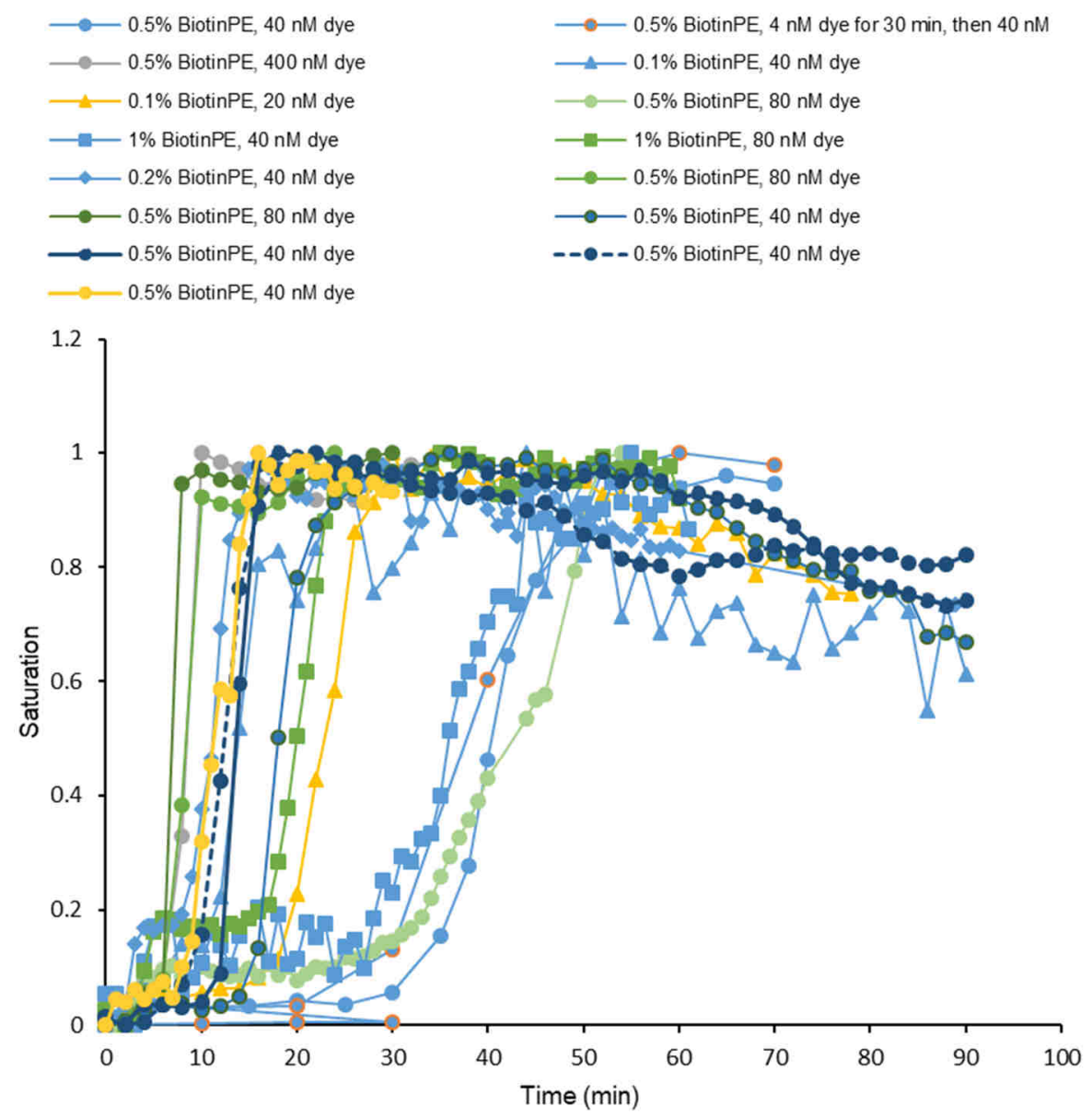

Figure 3.5 | Binding curves of ATTO 565-biotin to SAv-coated SLBs. Biotin percentages in the SLBs and concentration of ATTO 565-biotin were varied. Saturation values were derived from fluorescence measurements.

We explored two possible mechanisms for the observed loss of analyte. If this depletion is caused by nonspecific binding of the dye to a surface or by nonspecific binding of SAv where it can still bind the biotinylated dye, then the transport delay depends on the concentration of the dye, but does not depend on the biotin density in the SLBs. On the other hand, if the vesicles that form the SLBs also adhere to surfaces other than the flow cell surface and subsequently bind SAv, the delay depends on both the concentration of dye and 
the biotin\% in the SLBs. We explored both possible explanations by studying the dependencies of $t_{1 / 2}$, as shown in Figure 3.6. With decreasing concentration of ligand, $t_{1 / 2}$ increased, but $t \frac{1}{2}$ also increased with increasing biotin density in the SLB. Therefore, $t_{1 / 2}$ appears to correlate with the ratio of ligands per receptor. Because the Tygon Microbore tubing S-54-HL that we used is hydrophobic, this implies that a lipid monolayer is formed on the inside of the tubing.
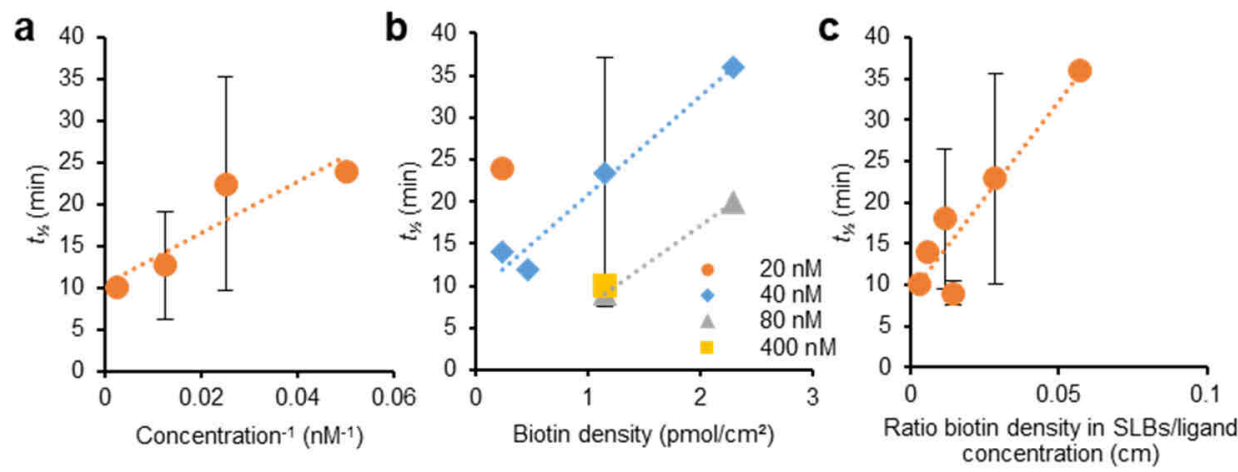

Figure 3.6| Possible correlations for $t \frac{1}{2}$ derived from time-dependent fluorescence measurements (Figure 3.5). a) $t \frac{1 / 2}{2}$ as function of the concentration of ATTO 565-biotin in solution. b) $t \frac{t}{2}$ as function of the density of biotin-DOPE in the SLBs for different concentrations of ATTO 565-biotin in solution. c) $t \frac{1}{2}$ as function of the ratio of the concentration of ATTO 565-biotin in solution over the density of biotin-DOPE in the SLBs.

To simulate the possible modes of depletion of species from solution and study the influence of various variables on the binding of ligands to the surface of the microchannel, we constructed a 2D finite element simulation in COMSOL. This model describes a stationary laminar flow of water through a tube, a hole (representing the hole in the PDMS into which the tubing is inserted), a channel and another hole, each represented by a rectangle (Figure 3.7a). At $t=0$, an inflow of a dilute species is started and the transport of this species through the flow is simulated using flow coupling multiphysics. This species can react with binding sites at the boundaries in the form of a chemical equilibrium according to the Langmuir adsorption model. The bottom surface of the channel has four electrodes, which are defined as non-reactive edges of $500 \mu \mathrm{m}$, and three corral areas with SLBs, which are defined as reactive edges of $500 \mu \mathrm{m}$. Figure 3.7b shows an example of the simulation of molecular transport in this model. 
a

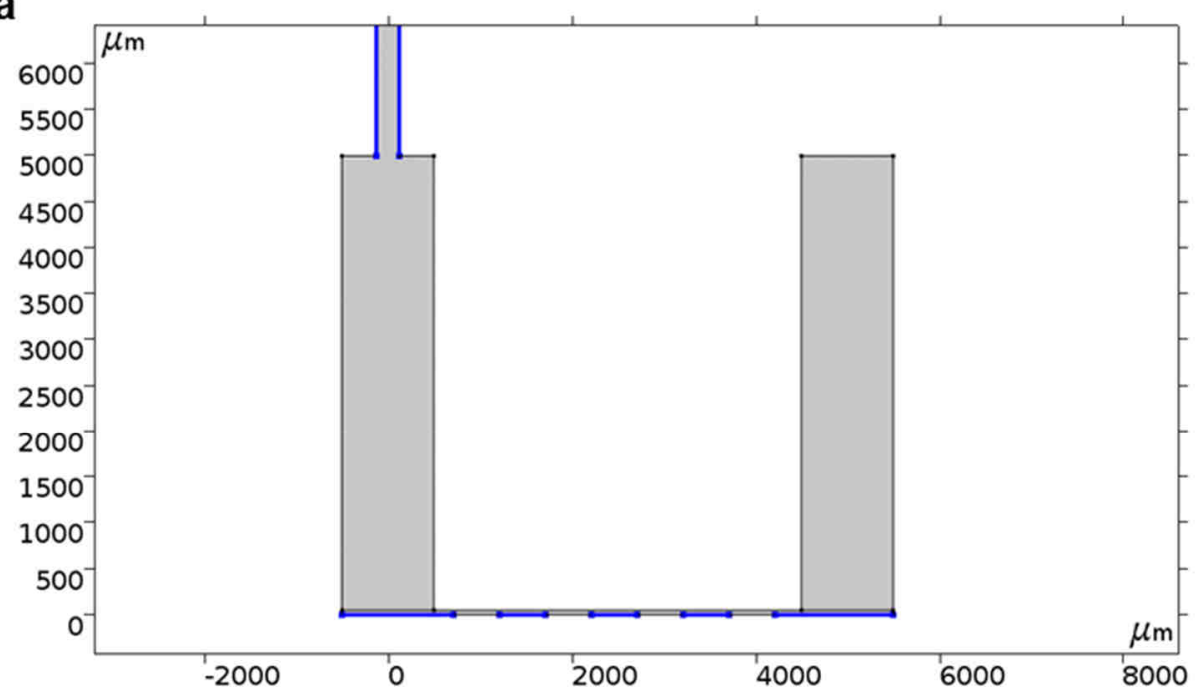

b

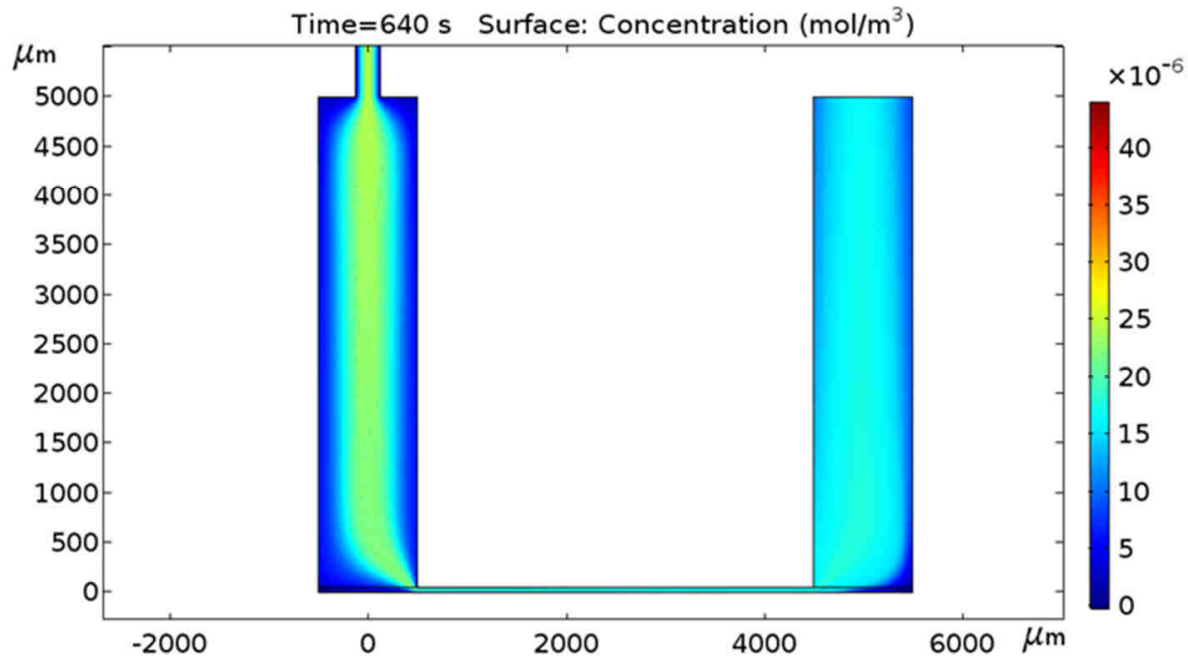

Figure 3.7| Snapshots of the 2D model in COMSOL. a) Snapshot of the geometry. The edges representing reactive surfaces are indicated in blue. b) Snapshot of the concentrations in the microchannel in a simulation of species transport through the channel with reactive surfaces along the tube and bottom. Density of binding sites $\Gamma_{\mathrm{s}}=$ $1 \mathrm{pmol} / \mathrm{cm}^{2}$, inflow concentration of species c $c_{0, c}=40 \mathrm{nM}$, flow rate $\phi_{\text {in }}=10 \mu \mathrm{l} / \mathrm{min}$, length of tubing $I=700 \mathrm{~mm}$. 
To approximate the adsorption to a tube surface in a $2 \mathrm{D}$ model, we used a density of binding sites on the "tube" edges that was a factor 2 higher than on the "glass" edges. This factor 2 is the difference in circumference between a pair of parallel plates and a tube that have equal cross sections.
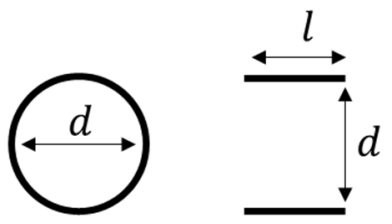

Figure 3.8| Dimensions of a round and rectangular cross-section.

A tube with diameter $d$ has a circumference of $\pi d$ and a cross section of $\frac{\pi}{4} d^{2}$ (Figure 3.8). A 2D simulation with two edges at a distance $d$ may be regarded as a projection of tube with rectangular cross section having two nonreactive sides of length $d$ and two reactive sides of length $l$. The rectangular cross section is $I \cdot d$. If both tubes have the same cross section $l \cdot d=\frac{\pi}{4} d^{2}$, then the length of the reactive sides $2 l=\frac{\pi}{2} d$. This is half of the circumference of the tube with circular cross section. Because saturation of the "tube" edges is reached, the difference in diffusion rates from the center of the tube to the edge can be ignored. In this model, we investigated the influence of nonspecific adsorption to the tubing. We simulated the binding of a species with a diffusion coefficient $D=4 \cdot 10^{-6} \mathrm{~cm}^{2} \mathrm{~s}^{-1}$, which is typical for ATTO dyes, at a concentration of $40 \mathrm{nM}$, to a surface with a density of binding sites, $\Gamma=1 \mathrm{pmol} \cdot \mathrm{cm}^{-2}$. Figure 3.9 shows how the average surface concentration at position $A$ develops over time as the dilute species is depleted by different reactive edges. 


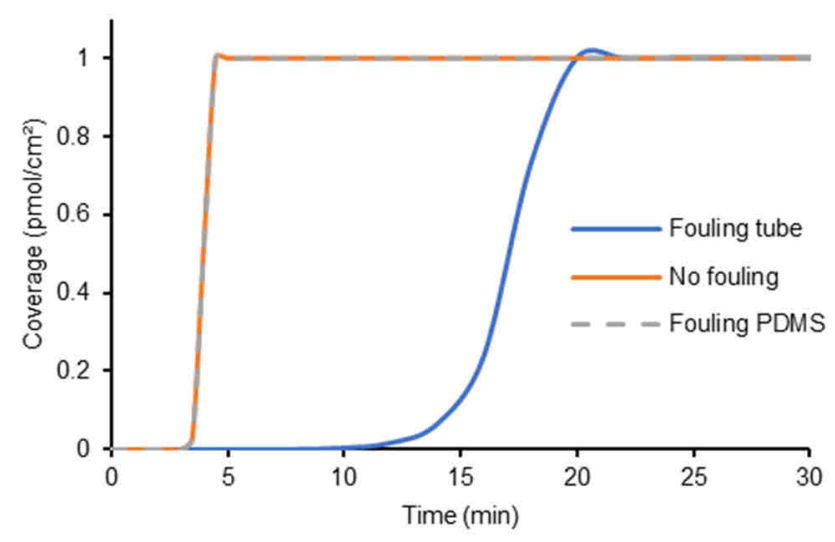

Figure 3.9| Development of the surface concentration of adsorbed species over time. Shown is the coverage in the first glass area between electrodes in the COMSOL simulation with analyte adsorption on the surface of the tubing and on the PDMS, and without such adsorption.

When only the "glass" edges are reactive, the surface coverage increases rapidly between 3 and $4 \mathrm{~min}$. When the "tube" edges are also reactive with the same density of binding sites, the binding of the ligand is delayed and occurs over a longer time. Both the shape and delay agree well with the experimental binding curves shown in Figure 3.4 and Figure 3.5. In the case of reactive "tube" edges, the effect on $t \frac{1}{2}$ of varying the concentration of ligand or varying the density of binding sites on the "tube" edges and on the "glass" edges is the same (Figure 3.10).

If the "PDMS" edges are also reactive, the profile is almost identical to the case of "glass" alone, indicating that fouling on PDMS does not significantly affect the rate of transport towards the surface of the microchannel. The surface area of the PDMS is approximately equal to that of the tubing, but in the 2D simulation the "PDMS" edges are only $10 \%$ of the "tubing" edges. Yet the difference in simulated surface area alone cannot account for the absence of transport delay. Instead, this can be understood from Figure $3.7 \mathrm{~b}$, which shows that the velocity difference between the center and edge of the hole leads to a higher concentration in the microchannel than at the edge of the hole. The residence time in the center is approximately $4 \mathrm{~s}$, allowing a mean squared displacement of $0.1 \mathrm{~mm}$, which is only a fifth of the radius of the hole. Since the 
surface reactions depend on concentration, no significant depletion at the edges of the hole are to be expected before the transported species reach the microchannel. This remains valid as long as the flow rate is higher than 2 $\mu \mathrm{L} / \mathrm{min}$.

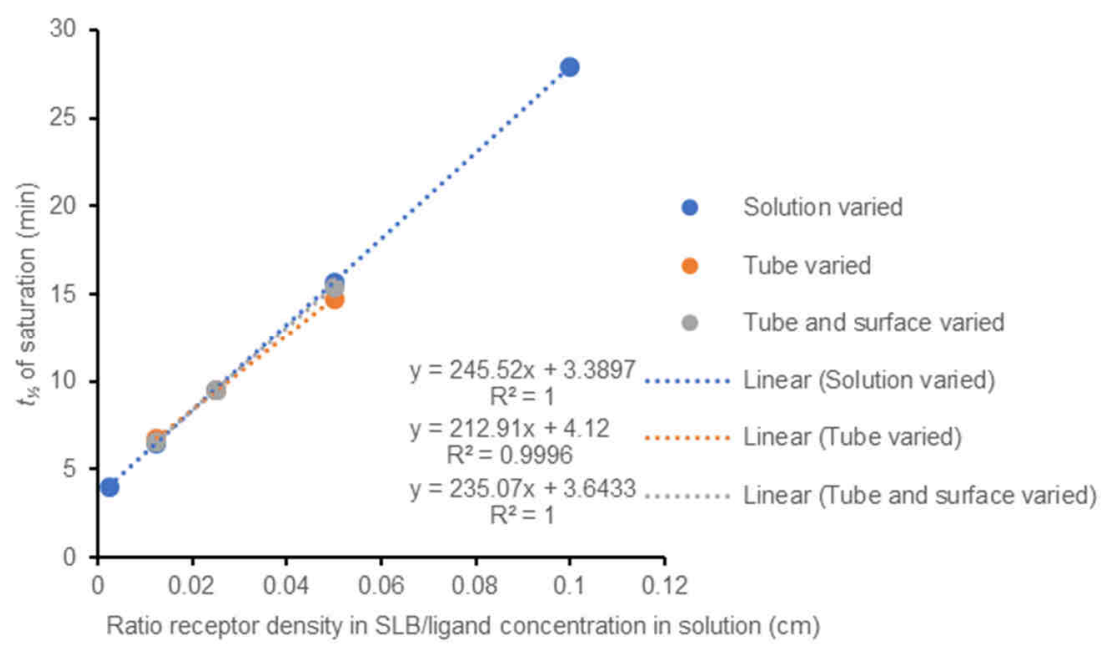

Figure 3.10 Influence of varying solution concentration and density of binding sites on the time needed to reach a saturation of $50 \%$ in COMSOL simulation. Where constant, the receptor density in the SLB is $1 \mathrm{pmol} / \mathrm{cm}^{2}$ and the ligand concentration in solution is $40 \mathrm{nM}$. Because the biotin density in the SLB is $1 \mathrm{pmol} / \mathrm{cm}^{2}$, the $x$ scale may also be interpreted as ligand concentration ${ }^{-1}\left(\mathrm{nM}^{-1}\right)$ in the case of the "solution varied" points. Varying the biotin\% in the SLBs or the concentration of ligand appears to have the same effect.

Figure 3.11 shows $t \frac{1 / 2}{2}$ as function of the ratio of receptors to ligands. A simulation that assumes ligand binding to the surface of an $800 \mathrm{~mm}$ long tube before binding to the sensor surface follows the slope of the trendline but has an offset that is $5 \mathrm{~min}$ lower. The slope of the simulated trend confirms that depletion of ATTO 565-biotin from the solution caused by binding to the tubing can explain the observed delay of binding to the SLBs and that the density of binding sites on the tubing is related to the biotin density in the SLBs. The higher offset of the experimental trend may be due to a slow buildup of pressure between the start of the syringe pump and the actual flow of the solution. 


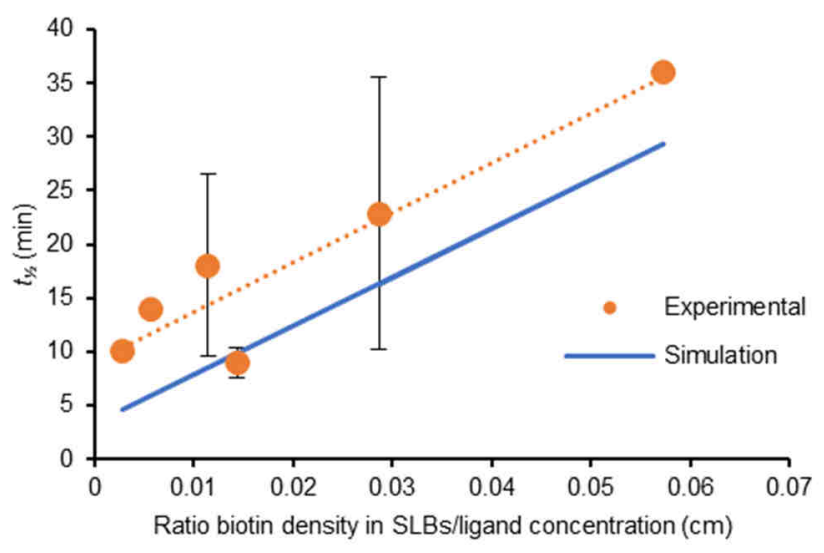

Figure 3.11| The saturation half-life, $t \frac{1}{2}$, as function of the ratio of biotin density in the SLBs and the concentration of ATTO 565-biotin in solution.

3.2.3 Dual inlet flow cell to avoid nonspecific binding

Because the tubing binds a large amount of the species of interest but is essential for the function of the microfluidic device and cannot be replaced without risk of disrupting the SLBs by exposure to air. To mitigate this problem, we designed a flow cell with two inlets.

Dual inlet flow cells are commonly applied for applications that involve mixing or separation, ${ }^{18-20}$ but have also been used to provide shear flow that prevents cell sedimentation. ${ }^{21}$ We used the dual inlet to uncouple the SLB formation process from the sensing step.

The dual inlet flow cell has a straight microchannel of $6 \mathrm{~mm}$ length with an arching secondary inlet (together resembling the letter " $r$ ", Figure 3.2c). With this shape, the distance between inlets is maximized to avoid tearing the PDMS during the fabrication of the flow cell. We used the curved inlet (inlet 2) for the assembly of the SLBS and SAv, and the straight inlet (inlet 1) for the flow of ligands (ATTO 565-biotin and nanoparticles), to avoid extra disturbances that may be caused by turns in the channel. To prevent flow from one inlet to the other, a buffer flow is maintained through the inactive inlet at one tenth of the flow rate through the active inlet. This way, the analytes do not encounter the tubing with adsorbed vesicles and SAv before reaching the microchannel. 
When the dual inlet flow cell was used for time-dependent binding studies of ATTO 565-biotin, we found that its binding profiles matched the simulated profiles for a scenario without nonspecific binding (Figure 3.12). This means that $t_{1 / 2}$ depends only on the volume of the tubing and confirms that the dual inlet design effectively prevents the formation of a sink of analyte at the inner tubing surface. It also further confirms that nonspecific adsorption of the vesicles, rather than of ATTO 565-biotin itself, caused the depletion of the dye.

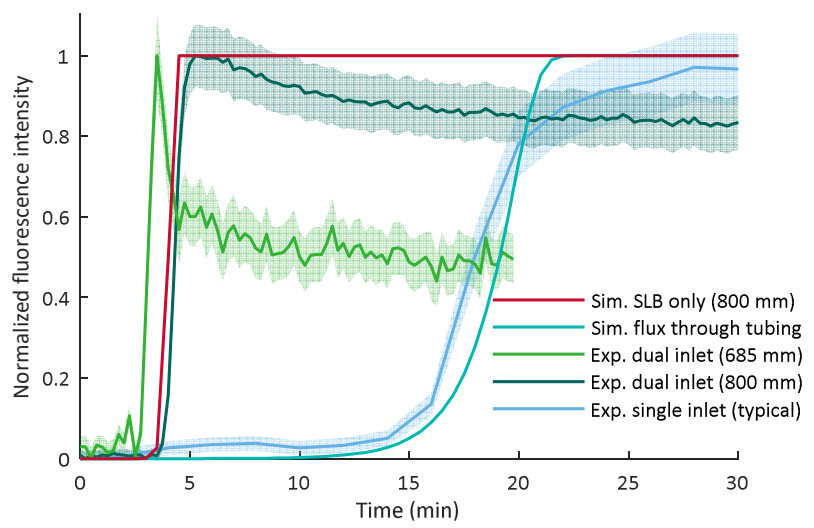

Figure 3.12 | Time-dependent binding profiles of ATTO 565-biotin to labelled SAv. All binding profiles are for $40 \mathrm{nM}$ ATTO 565 -biotin in solution and $0.5 \%$ biotin in the SLBs. Nonspecific binding is simulated as flux through the "tubing" edges with a density of binding sites twice as high as on the SLB to account for the circular cross-section. Shaded areas represent the standard errors between corrals. The decreasing fluorescence intensity after the maximum is due to photobleaching.

\subsubsection{Time-dependent binding of nanoparticles}

The adsorption kinetics and mass transport of nanoparticles are often significantly different compared to small molecules. To study the binding of nanoparticles experimentally, we used SAv-coated fluorescent beads of 400 $\mathrm{nm}$. The beads were passed through single-inlet microchannels at a concentration of $10 \mu \mathrm{g} / \mathrm{mL}$ at varying flow rates over SLBs containing $0.2 \%$ biotin-DOPE, while fluorescence micrographs were acquired at regular intervals (Figure 3.13a and b). At a flow rate of $5 \mu \mathrm{L} / \mathrm{min}$, the fluorescence on the SLBs increased 5 times faster than at a flow rate of $1 \mu \mathrm{L} / \mathrm{min}$, meaning that the same fraction of particles that passed through the flow cell bound to the sensing area. 

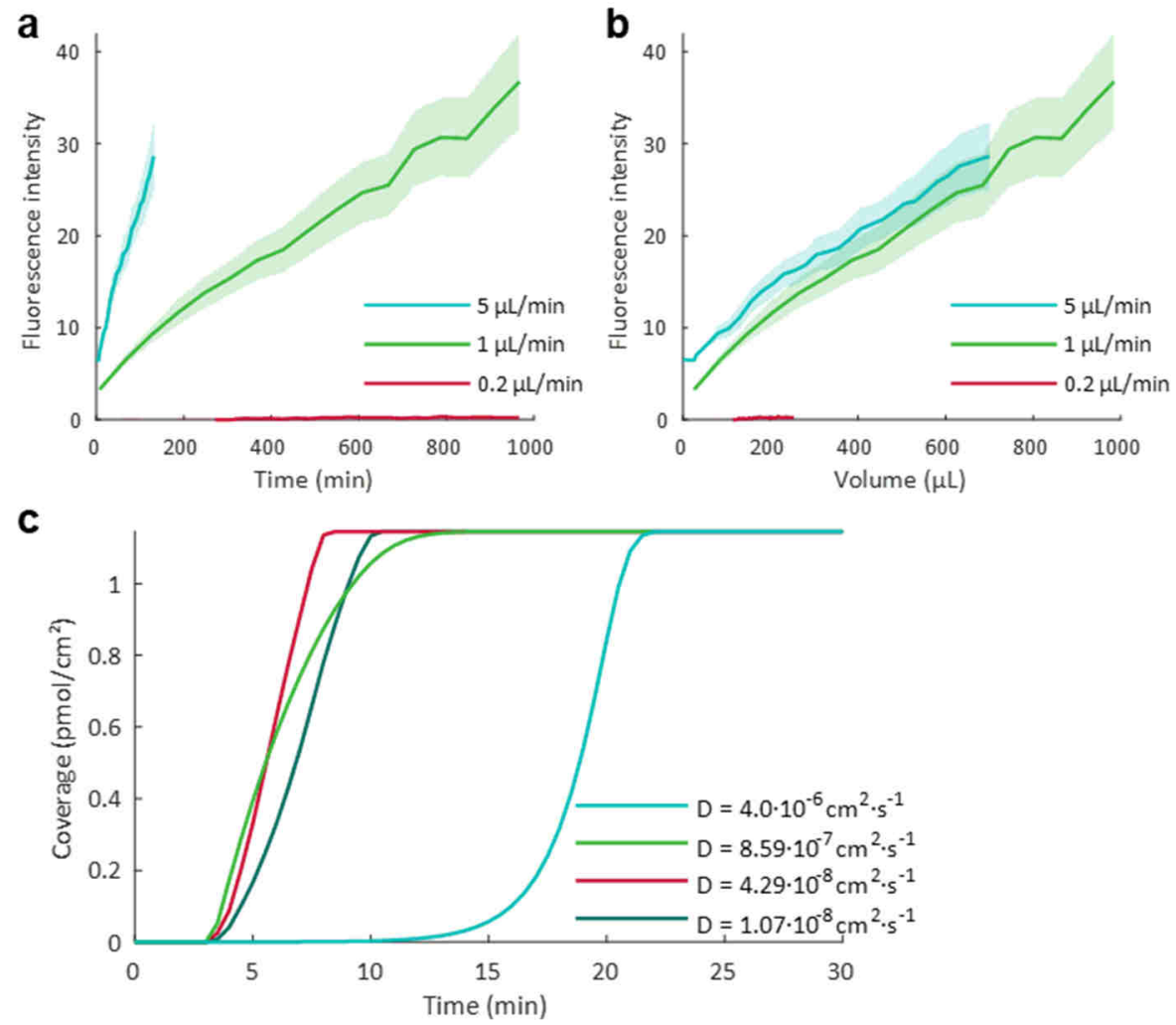

Figure 3.13 | Experimental and simulated binding profiles of nanoparticles. a) Binding of SAv-coated fluorescent beads of $400 \mathrm{~nm}$ on SLBs containing $0.2 \%$ biotin-DOPE as function of time for different flow rates. Shaded areas represent the standard errors between corrals. b) The same binding profiles as function of volume, which corrects for the differences in flow rate. c) Simulation of the time-dependent binding of particles of varying size with adsorption in the tubing, assuming a diffusion coefficient $D=4.0 \cdot 10^{-6}$ $\mathrm{cm}^{2} \cdot \mathrm{s}^{-1}$ for small molecules, $D=8.6 \cdot 10^{-7} \mathrm{~cm}^{2} \cdot \mathrm{s}^{-1}$ for $5 \mathrm{~nm}$ proteins, $D=4.3 \cdot 10^{-8} \mathrm{~cm}^{2} \cdot \mathrm{s}^{-1}$ for $100 \mathrm{~nm}$ particles and $D=1.1 \cdot 10^{-8} \mathrm{~cm}^{2} \cdot \mathrm{s}^{-1}$ for $400 \mathrm{~nm}$ particles. Concentration of particles is $40 \mathrm{nM}$ and flow rate $10 \mu \mathrm{L} / \mathrm{min}$.

At a flow rate of $0.2 \mu \mathrm{L} / \mathrm{min}$, however, no significant increase in fluorescence was observed, even after $16 \mathrm{~h}$, during which more than $200 \mu \mathrm{L}$ of the nanoparticle suspension was passed over the SLBs.

The influence of receptor density on the binding of SAV-coated beads was investigated with electrophoretic biotin-DOPE gradients, where Texas Red 1,2- 
dihexadecanoyl-sn-glycero-3-phosphoethanolamine (TexasRed-DHPE) functioned as an indicator of biotin density (Figure 3.14). The effect of biotin density can only be observed at very low densities (Figure 3.14d). At higher densities, the particles can bind anywhere and are only limited by mass transport and geometrical constraints.
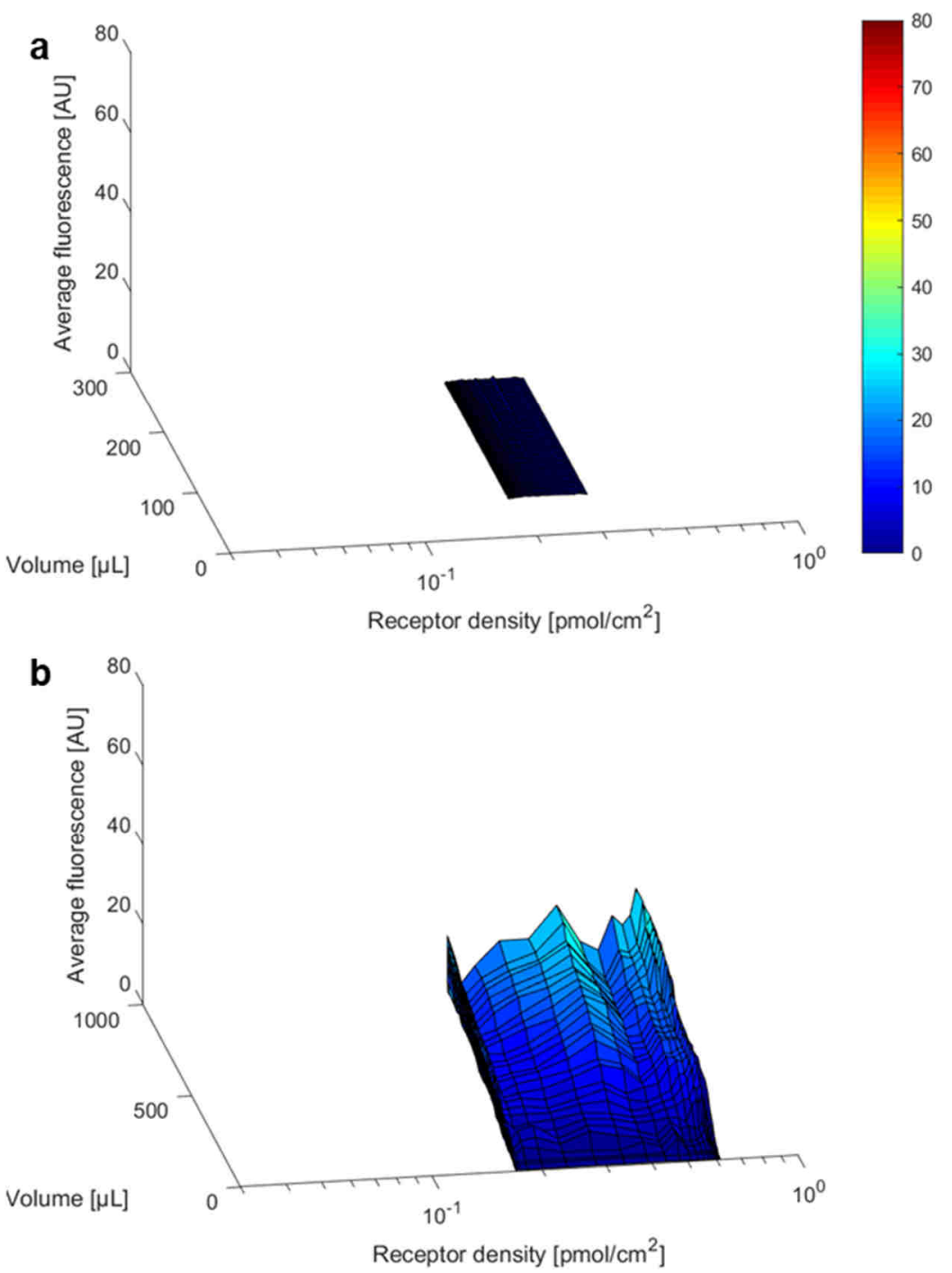

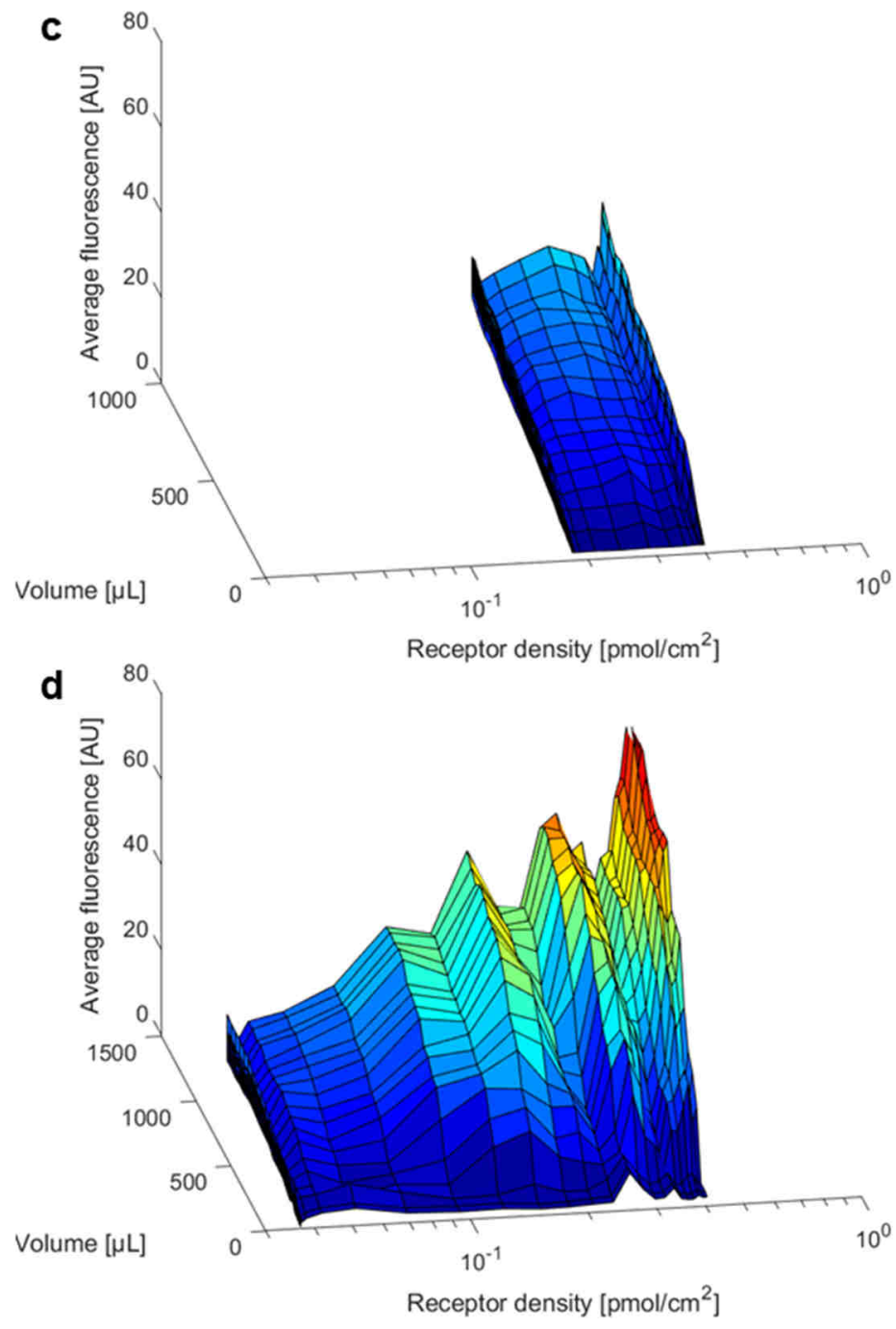

Figure 3.14| Binding of 400-nm, streptavidin-coated beads to biotinylated SLBs on 430 $\mu \mathrm{m}$ corrals as function of the biotin density and the volume of particle solution that was passed through the microchannel. The corrals were cropped from fluorescent micrographs and were divided into bins of equal size. The average fluorescence intensity (gray value) in each bin was determined for each time point. Flow rate was: a) $0.2 \mu \mathrm{L} / \mathrm{min}$, b) $5 \mu \mathrm{L} / \mathrm{min}, \mathbf{c}, \mathbf{d}) 1 \mu \mathrm{L} / \mathrm{min}$. SLBs were made with a,b,c) $0.2 \%$ biotin-DOPE, d) $0.05 \%$ biotin-DOPE. The particle concentration was $10 \mu \mathrm{g} / \mathrm{mL}$. 
To apply the COMSOL model to proteins and nanoparticles we investigated the behavior of the model at varying diffusion coefficients and very low concentrations. The model does not explicitly account for occupied area on the surface or the inertia of particles. When particles are transported in laminar flow, but not in the Stokes flow regime, they are subject to migration and focusing under inertial lift forces if the particle Reynolds number $R_{p} \geq 1$, where

$R_{p}=\operatorname{Re}\left(\frac{a}{h}\right)^{2}=\frac{\rho U a^{2}}{\mu h}$

with $a$ the particle diameter, $h$ the channel height, $U$ the average flow velocity, and $\rho$ and $\mu$ the density and viscosity of the medium. ${ }^{22}$ In our system, where $h$ is not smaller than $50 \mu \mathrm{m}$, particles are no larger than $400 \mathrm{~nm}$ and the flow velocity is no greater than $1 \mathrm{~m} / \mathrm{s}$, the particle Reynolds number is never larger than $R_{p}=0.01$. Therefore, proteins, viruses and nanoparticles may be considered as small molecules when accounting for inertia, only with a different diffusion coefficient.

To estimate the diffusion coefficient of a typical protein, a typical virus, and our fluorescent beads, we used the Stokes-Einstein equation:

$D=\frac{k_{B} T}{6 \pi \mu r}$

with $k_{\mathrm{B}}$ Boltzmann's constant, $T$ the absolute temperature, $\mu$ the viscosity of the medium and $r$ the radius of the particle. We calculated that $5 \mathrm{~nm}$ particles have a diffusion coefficient of $D=8.6 \cdot 10^{-7} \mathrm{~cm}^{2} \cdot \mathrm{s}^{-1}, 100 \mathrm{~nm}$ particles $D=4.3 \cdot 10^{-8}$ $\mathrm{cm}^{2} \cdot \mathrm{s}^{-1}$ and $400 \mathrm{~nm}$ particles $D=1.1 \cdot 10^{-8} \mathrm{~cm}^{2} \cdot \mathrm{s}^{-1}$. In Figure $3.13 \mathrm{c}$ we show the simulated binding profiles for particles with these diffusion coefficients and a dye if the density of binding sites is equal on the tubing and the SLBs and the flow rates and concentrations are the same. Interestingly, not only the onset of binding is much faster for the larger particles, but also the saturation is reached much earlier than for small molecules.

The faster onset in the time-dependent binding of nanoparticles may be understood from the concentration profile in the COMSOL simulation of small molecules (Figure 3.7b). Because the flow rate has a parabolic profile, the concentration in the narrow microchannel is primarily determined by the concentration in the faster-flowing center of the tube. For species with a lower 
diffusion coefficient or at higher flow rates, the concentration in the center of the tube is less affected by nonspecific binding to the tubing. The combination of the length and width of the tube, the diffusion constant and the flow rate can lead either to regime (1) where most of the species reaches the wall of the tube and binds there, or regime (2) where most of the species does not reach the wall and leaves the tubing unhindered. For our system, the $400 \mathrm{~nm}$ nanoparticles are in regime (2) at flow rates of $1 \mu \mathrm{L} / \mathrm{min}$ and higher, but in regime (1) at $0.2 \mu \mathrm{L} / \mathrm{min}$, whereas ATTO 565-biotin is in regime (1) even at 10 $\mu \mathrm{L} / \min$.

The intersecting binding profiles in Figure 3.13c indicate that the diffusion coefficient has opposing influences on the time-dependent binding. At low diffusion coefficients, some of the particles in the middle of the tubing do not reach the sides while in the tubing, but do reach the sensing area, where they are responsible for the early onset of binding. In the microchannel, low diffusion coefficients become a disadvantage, leading to less efficient binding in the sensing area. At $10 \mu \mathrm{g} / \mathrm{mL}$ of $400 \mathrm{~nm}$ particles, their molar concentration is approximately $8.8 \cdot 10^{-4} \mathrm{nM}$. With a flow rate of $1 \mu \mathrm{L} / \mathrm{min}$ and without nonspecific binding in the tubing, the coverage of binding sites after $16 \mathrm{~h}$ for $400 \mathrm{~nm}$ particles is $0.042 \%$. This corresponds to a geometrical coverage of $32 \%$. At such high coverages, the area that is occupied by particles cannot be ignored. The validity of this finite element simulation is therefore limited to situations where the available area is much larger than the projected area of the particles. The linear binding profiles in Figure 3.12a and b indicate, however, that the surface coverage was not yet approaching saturation. As a result, the particles with diffusion constant $D=4.3 \cdot 10^{-8} \mathrm{~cm}^{2} \cdot \mathrm{s}^{-1}$ in Figure $3.13 \mathrm{c}$ can reach saturation faster than either smaller or larger particles.

\subsection{Conclusions}

We aimed to predict the binding of small molecules and nanoparticles as model analytes to surface receptors immobilized on the sensing area in a microfluidic device. Hereto, we studied the binding of a biotinylated dye to immobilized streptavidin and the binding of streptavidin-coated nanoparticles to immobilized biotin. The receptors were presented in a gradient to evaluate the combined effects of flow rate and receptor density. We found that the time until the small-molecule analyte started binding at the sensing area in the 
microchannel was strongly influenced by the extent of nonspecific binding of receptors in the tubing. For the nanoparticles, which have a lower diffusion coefficient, the onset of binding was faster, but binding to the tubing still affected the binding profiles. We presented a flow cell with two inlets that avoids the influence of nonspecific binding of receptors in the tubing on the time-dependent binding of ligands.

The time-dependent binding of small-molecule and nanoparticle analytes in microfluidic sensors is strongly influenced by the dimensions and surface chemistry of the parts that are traversed before reaching the sensing area. When nonspecific binding can disturb the sensing, shorter and wider inlets are favorable, but more thorough avoidance of binding outside the sensing area can be achieved by separating sensing and assembly steps over different inlets. This method allows the fabrication of microfluidic sensors with receptors on an antifouling biomimetic surface with minimum loss of analyte.

\subsection{Acknowledgements}

Erik Hamming is thanked for his part in the data analysis and editing, and for writing the MATLAB scripts, Wouter Vijselaar for the fabrication of the chips, Mark Verheijden and Jasper van Weerd for their help with the flow cells, and Melissa Goodwin for proofreading.

\subsection{Experimental}

\subsubsection{Reagents}

All starting materials and chemicals were purchased from Sigma-Aldrich, Fluka, Serva, Becton Dickinson, Avanti Polar Lipids, Acros organics, Microchem, Biotium, Thermo Fisher, Spherotech, Schott and Fujifilm, and they were used as received, unless otherwise stated.

\subsubsection{Chip fabrication}

Flow cells were fabricated according to the procedure described earlier in. ${ }^{13} \mathrm{~A}$ bilayer lift-off recipe was used for fabricating Au electrodes on Mempax glass wafers (Schott). First, LOR 5A (MicroChem) was spin-coated, after which normal lithography was performed on top with Olin OiR 907-17 photoresist (FujiFilm) to create a bilayer resist stack. Electrode patterns were made by exposing the 
photoresist through a patterned photomask and developing in Olin OPD 4262 (Fujifilm). The develop step washed away the exposed photoresist, and etching through the LOR 5A layer created an undercut. Then, $5 \mathrm{~nm}$ Ti and $95 \mathrm{~nm} \mathrm{Au}$ were deposited via e-beam evaporation (BAK 600, Balzers). The bilayer resist was then removed by sonication in acetone $(20 \mathrm{~min}$ ) and isopropanol (10 $\mathrm{min}$ ) followed by $5 \mathrm{~min}$ immersion in OPD 4262, serving as a sacrificial layer to leave patterned Au electrodes on borofloat glass. To fabricate the $\mathrm{Cr}$ corrals $(10 \mathrm{~nm}$ thick) in between the Au electrodes, the same procedure was performed a second time, but in this case following alignment with respect to the $\mathrm{Au}$ electrodes.

\subsubsection{PDMS flow channel}

Silicon flow channel masters were produced by standard photolithography steps and deep reactive ion etching. The polydimethylsiloxane (PDMS) flow channels were prepared from a degassed mixture of 10:1 Sylgard 184 elastomer and curing agent (Dow Corning Corp), which was cast onto the silicon master and cured at $60^{\circ} \mathrm{C}$ overnight. The flow channels were cut to size and inlets and outlets were punched using a $1 \mathrm{~mm} \varnothing$ punch (Harris Uni-core, Sigma-Aldrich). After bonding to the chip, a flow channel was prepared.

\subsubsection{PDMS bonding}

Chips were rinsed and sonicated extensively with acetone, ethanol and MilliQ water, and dried prior to UV-ozone exposure (UV/Ozone Procleaner plus, Bioforce Nanosciences) for at least 20 min. After UV exposure, the chips were rinsed with ethanol, water and dried under a stream of nitrogen. Both cut-out PDMS flow channels and cleaned chips were treated with oxygen plasma for 30 $\mathrm{S}$ at $40 \mathrm{~W}$ (Plasma prep II, SPI supplies) after which they were bonded immediately. The chips were placed on a hot plate for $10 \mathrm{~min}$ at $70^{\circ} \mathrm{C}$ to increase the binding strength. Tygon Microbore tubing S-54-HL (VWR, $0.25 \mathrm{~mm}$ inner $\varnothing$ and $0.75 \mathrm{~mm}$ outer $\varnothing$ ) was inserted into the PDMS. The assembled $\mu$ SLB electrophoresis chip was placed in an oven at $60^{\circ} \mathrm{C}$ for $1 \mathrm{~h}$. Leak-free operation was shown for flow rates up to $200 \mu \mathrm{L} / \mathrm{min}$.

\subsubsection{Vesicle preparation}

MPPC (1-myristoyl-2-palmitoyl-sn-glycero-3-phosphocholine, Avanti) was stored as a $10 \mathrm{mg} / \mathrm{mL}$ stock solution in chloroform at $-20^{\circ} \mathrm{C}$. Biotin-DOPE (1,2- 
dioleoyl-sn-glycero-3-phosphoethanolamine-N-(cap biotinyl), Avanti) was stored as $0.2 \mathrm{mg} / \mathrm{mL}$ solution and TR-DHPE (Texas Red ${ }^{\mathrm{TM}}$ 1,2-dihexadecanoyl-snglycero-3-phosphoethanolamine, Thermo Fisher) as $0.1 \mathrm{mg} / \mathrm{mL}$ solution in methanol. Molar ratios of 99-99.9\% MPPC, $0.1-1 \%$ biotin-DOPE and $0-0.2 \%$ TRDHPE were mixed in a glass vial, dried under a flow of nitrogen and kept under vacuum for at least $1 \mathrm{~h}$. The resulting film was resuspended by vortexing in MilliQ water at $50^{\circ} \mathrm{C}$ to form multilamellar vesicles (MLVs) at $1 \mathrm{mg} / \mathrm{mL}$. The MLV solution was extruded 11 times through a $100 \mathrm{~nm}$ polycarbonate membrane (Avanti) at $50^{\circ} \mathrm{C}$. The resulting large unilamellar vesicles (LUVs) were kept at room temperature and used within one week.

\subsubsection{SLB formation}

Before SLB formation, the flow cells were washed with 2\% SDS (Sigma-Aldrich), then rinsed with MilliQ water, and washed overnight with $2 \%$ Hellmanex $^{\mathrm{TM}}$ to activate the glass surface. Flow cells were mounted onto a heating plate using Scotch ${ }^{\mathrm{TM}}$ tape and rinsed with MilliQ water and PBS, while heating to $50^{\circ} \mathrm{C}$. Shortly before LUV incubation, the LUV solution was diluted to $0.5 \mathrm{mg} / \mathrm{mL}$ with PBS. The diluted LUV solution was passed through the flow cells for $30 \mathrm{~min}$ (10 $\mu \mathrm{L} / \mathrm{min}$ ) to allow adsorption and rupture of the vesicles on the chips. The chips were then washed with MilliQ water $(100 \mu \mathrm{L} / \mathrm{min})$, after which freshly prepared $0.5 \mathrm{mM}$ hydroxymethylferrocene ( $\mathrm{FcMeOH}, \mathrm{Acros})$ was passed through the device at the same flow rate. A potential difference of $2.0 \mathrm{~V}$ was applied over the device for $30 \mathrm{~min}$ to induce electrophoresis in the SLB. Subsequently, the chips were cooled rapidly on a heat exchanger to fix the surface gradient. The flow cells were then rinsed with MilliQ water and PBS. During all steps, care was taken to ensure that no air bubbles entered the flow cell.

\subsubsection{ATTO 565-biotin binding studies}

SLBs comprised of $99.0 \%-99.9 \%$ MPPC and $0.1 \%$ - 1\% biotinPE were incubated with $0.02 \mathrm{mg} / \mathrm{mL}$ labelled streptavidin (SAv) for at least $25 \mathrm{~min}(10 \mu \mathrm{L} / \mathrm{min})$. The SAv solutions were obtained by diluting solutions of SAv labelled with CF350 (Biotium) or Alexa Fluor 488 (Thermo Fisher) to $20 \mu \mathrm{g} / \mathrm{mL}$ with PBS. Subsequently, the chips were washed with PBS $(10 \mu \mathrm{L} / \mathrm{min})$. The SAv-modified SLBs were inspected with fluorescence microscopy at 10x magnification on an Olympus inverted IX71 epi-fluorescence research microscope with X-cite 120 PC as light source and a digital Olympus DR70 camera for image acquisition. For 
CF350, violet excitation ( $360 \leq \lambda_{\mathrm{ex}} \leq 370 \mathrm{~nm}$ ) and blue emission ( $440 \leq \lambda_{\mathrm{em}} \leq 480$ $\mathrm{nm}$ ) was filtered using the U-MWG2 Olympus filter cube. For Alexa Fluor 488, blue excitation ( $460 \leq \lambda_{\text {ex }} \leq 490 \mathrm{~nm}$ ) and green emission $\left(505 \leq \lambda_{\mathrm{em}} \leq 545 \mathrm{~nm}\right)$ was filtered. Binding of ATTO 565-biotin was monitored using green excitation $\left(510 \leq \lambda_{\mathrm{ex}} \leq 550 \mathrm{~nm}\right)$ and red emission $\left(\lambda_{\mathrm{em}}>590 \mathrm{~nm}\right)$. The streptavidin-modified SLBs were incubated with $20-400 \mathrm{nM}$ ATTO 565-biotin in PBS at a constant flow rate of $10 \mu \mathrm{L} / \mathrm{min}$, which was the highest flow rate that did not disturb the modified surface in earlier studies, ${ }^{13}$ while simultaneously fluorescence micrographs were acquired at regular intervals.

\subsubsection{Data analysis}

MATLAB was used in the analysis of the data. AnalyseFluoresData_v2.m and folderToExcel.m are custom-written scripts, while ginputc.m was downloaded from the MathWorks File Exchange and used without modification. AnalyseFluoresData_v2.m aligns micrographs using a Sobel edge-detection algorithm, after which the user can manually position a mask with the chips features to the micrograph and designate an area to take as background. The program then crops the micrograph into corrals and saves them to a folder. folderToExcel.m takes a folder with corral as input and outputs the backgroundcorrected intensities per column of pixels to an excel-file in the input folder.

\subsubsection{Nanoparticle binding studies}

SLBs comprised of $99.4 \%-99.9 \%$ MPPC, $0.05 \%-0.5 \%$ biotinPE and $0.05 \%-0.2 \%$ TR-DHPE were prepared. The SLBs were inspected with fluorescence microscopy, using green excitation and red emission filters, and then incubated with SAv-coated yellow fluorescent polystyrene beads of $0.4 \mu \mathrm{m}$ (Spherotech) or first incubated with SAv solution, then washed with PBS and subsequently incubated with biotin-coated yellow fluorescent polystyrene beads of $0.15 \mu \mathrm{m}$ (Spherotech). The SAv solutions were obtained by diluting solutions of SAv labelled with CF350 or Alexa Fluor 647 (Thermo Fisher) to $20 \mu \mathrm{g} / \mathrm{mL}$ with PBS. The fluorescent polystyrene beads were diluted with PBS to $10 \mu \mathrm{g} / \mathrm{mL}$ before use. For the yellow fluorescent polystyrene beads, blue excitation and green emission were used. For Alexa Fluor 647, red excitation ( $630 \leq \lambda_{\text {ex }} \leq 650 \mathrm{~nm}$ ) and far red emission $\left(670 \leq \lambda_{\text {em }} \leq 690 \mathrm{~nm}\right)$ was filtered. 


\subsubsection{Finite element simulations}

Finite element simulations were performed in COMSOL Multiphysics 5.3a with the Chemical Reaction Engineering Module, using Laminar Flow, Transport of Diluted Species, Chemistry and Surface Reactions Physics, and Flow Coupling Multiphysics in a 2D model. COMSOL finite element model and MATLAB scripts are available with Reference 23.

\subsection{References}

1. Xu, D., Huang, X., Guo, J. \& Ma, X. Automatic smartphone-based microfluidic biosensor system at the point of care. Biosens. Bioelectron. 110, 78-88 (2018).

2. Kammer, M. N., Kussrow, A. K., Olmsted, I. R. \& Bornhop, D. J. A Highly Compensated Interferometer for Biochemical Analysis. ACS Sensors 3, 1546-1552 (2018).

3. Zhang, R. Q., Hong, S. L., Wen, C. Y., Pang, D. W. \& Zhang, Z. L. Rapid detection and subtyping of multiple influenza viruses on a microfluidic chip integrated with controllable micro-magnetic field. Biosens. Bioelectron. 100, 348-354 (2018).

4. Liu, C., Xu, X., Li, B., Situ, B., Pan, W., Hu, Y., An, T., Yao, S. \& Zheng, L. Single-ExosomeCounting Immunoassays for Cancer Diagnostics. Nano Lett. 18, 4226-4232 (2018).

5. Schneider, A.-K. \& Niemeyer, C. M. DNA Surface Technology: From Gene Sensors to Integrated Systems for Life and Materials Sciences. Angew. Chem. Int. Ed. 57, 1695916967 (2018).

6. Walper, S. A., Lasarte Aragonés, G., Sapsford, K. E., Brown, C. W., Rowland, C. E., Breger, J. C. \& Medintz, I. L. Detecting Biothreat Agents: From Current Diagnostics to Developing Sensor Technologies. ACS Sensors 3, 1894-2024 (2018).

7. Chand, R. \& Neethirajan, S. Microfluidic platform integrated with graphene-gold nanocomposite aptasensor for one-step detection of norovirus. Biosens. Bioelectron. 98, 4753 (2017).

8. Cohen, L. \& Walt, D. R. Highly Sensitive and Multiplexed Protein Measurements. Chem. Rev. 119, 293-321 (2019).

9. Song, Y., Shi, Y., Huang, M., Wang, W., Wang, Y., Cheng, J., Lei, Z., Zhu, Z. \& Yang, C. Bioinspired Engineering of a Multivalent Aptamer-Functionalized Nanointerface to Enhance the Capture and Release of Circulating Tumor Cells. Angew. Chem. Int. Ed. 58, 2236-2240 (2019).

10. Sepúlveda, B., Río, J. S. del, Moreno, M., Blanco, F. J., Mayora, K., Domínguez, C. \& Lechuga, L. M. Optical biosensor microsystems based on the integration of highly sensitive Mach-Zehnder interferometer devices. J. Opt. A Pure Appl. Opt. 8, S561-S566 (2006).

11. Stroumpoulis, D., Haining, Z., Rubalcava, L., Gliem, J. \& Tirrell, M. Cell adhesion and growth to peptide-patterned supported lipid membranes. Langmuir 23, 3849-3856 (2007).

12. Van Weerd, J., Sankaran, S., Roling, O., Sukas, S., Krabbenborg, S., Huskens, J., le Gac, S., Ravoo, B. J., Karperien, M. \& Jonkheijm, P. A Microfluidic Device with Continuous Ligand Gradients in Supported Lipid Bilayers to Probe Effects of Ligand Surface Density and 
Solution Shear Stress on Pathogen Adhesion. Adv. Mater. Interfaces 3, 1600055 (2016).

13. Van Weerd, J., Krabbenborg, S. O., Eijkel, J., Karperien, M., Huskens, J. \& Jonkheijm, P. On-Chip Electrophoresis in Supported Lipid Bilayer Membranes Achieved Using Low Potentials. J. Am. Chem. Soc. 136, 100-103 (2014).

14. Krabbenborg, S. O., van Weerd, J., Karperien, M., Jonkheijm, P. \& Huskens, J. Locked-in Biomimetic Surface Gradients that are Tunable in Size, Density and Functionalization. ChemPhysChem 15, 3460-3465 (2014).

15. Mammen, M., Choi, S.-K. \& Whitesides, G. M. Polyvalent Interactions in Biological Systems: Implications for Design and Use of Multivalent Ligands and Inhibitors. Angew. Chem. Int. Ed. 37, 2754-2794 (1998).

16. Di lorio, D., Verheijden, M. L., van der Vries, E., Jonkheijm, P. \& Huskens, J. Weak Multivalent Binding of Influenza Hemagglutinin Nanoparticles at a SialoglycanFunctionalized Supported Lipid Bilayer. ACS Nano 13, 3413-3423 (2019).

17. Vahey, M. D. \& Fletcher, D. A. Influenza A virus surface proteins are organized to help penetrate host mucus. elife 8, 1-24 (2019).

18. Marimuthu, M., Kim, S. \& An, J. Amphiphilic triblock copolymer and a microfluidic device for porous microfiber fabrication. Soft Matter 6, 2200 (2010).

19. Xiong, B., Ren, K., Shu, Y., Chen, Y., Shen, B. \& Wu, H. Recent Developments in Microfluidics for Cell Studies. Adv. Mater. 26, 5525-5532 (2014).

20. Gao, D., Jin, F., Zhou, M. \& Jiang, Y. Recent advances in single cell manipulation and biochemical analysis on microfluidics. Analyst 144, 766-781 (2019).

21. Yun, H., Kim, K. \& Lee, W. G. Effect of a dual inlet channel on cell loading in microfluidics. Biomicrofluidics 8, 066501 (2014).

22. Amini, H., Lee, W. \& Di Carlo, D. Inertial microfluidic physics. Lab Chip 14, 2739-2761 (2014).

23. Overeem, N. J., Hamming, P. H. (Erik) \& Huskens, J. Time-Dependent Binding of Molecules and Nanoparticles at Receptor-Modified Supported Lipid Bilayer Gradients in a Microfluidic Device. ChemistrySelect 5, 9799-9805 (2020). 


\section{Chapter 4}

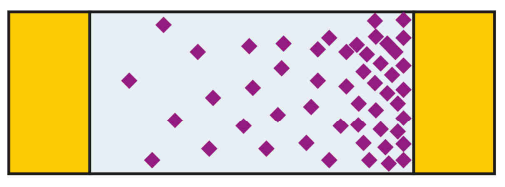

II

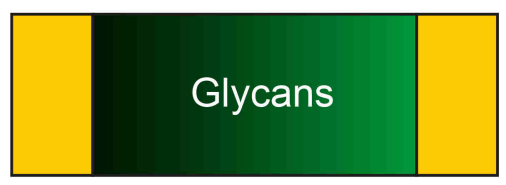

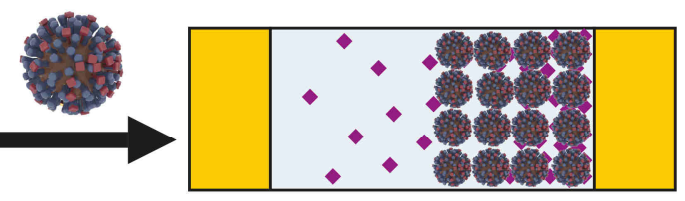

II

Threshold receptor density

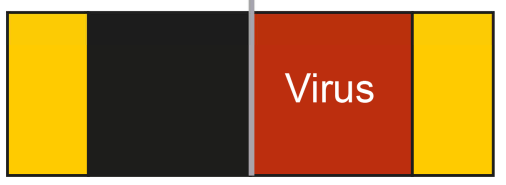

\section{Highlights}

- A new method is presented to quantify multivalent binding at receptor density gradients

- Influenza A virus PR8 binds superselectively to areas with high receptor density, showing in threshold in receptor density dependence

- The avidity of PR8 is higher for longer glycans

- The avidity decreases strongly with decreasing glycan length for human-type glycans, but less for avian-type ones

- A model is presented to calculate the number of virus-receptor interactions from virus binding profiles 


\section{Hierarchical multivalent effects control influenza host specificity}

Understanding how emerging influenza viruses recognize host cells is critical in evaluating their zoonotic potential, pathogenicity, and transmissibility between humans. The surface of the influenza virus is covered with hemagglutinin (HA) proteins that can form multiple interactions with sialic acid-terminated glycans on the host cell surface. This multivalent binding affects the selectivity of the virus in ways that cannot be predicted from the individual receptor-ligand interactions alone. Here we show that the intrinsic structural and energetic differences between the interactions of avian- or human-type receptors with influenza HA translate from individual site affinity and orientation through receptor length and density on the surface into virus avidity and specificity. We introduce a method to measure virus avidity using receptor density gradients. We found that influenza viruses attached stably to a surface at receptor densities that correspond to a minimum number of approximately $8 \mathrm{HA}$-glycan interactions, but more interactions were required if the receptors were short humantype. Thus, the avidity and specificity of influenza viruses for a host cell depend not on the sialic acid linkage alone but on a combination of linkage and the length and density of receptors on the cell surface. Our findings suggest that threshold receptor densities play a key role in virus tropism, which is a predicting factor for both their virulence and zoonotic potential.

Part of this chapter has been published in:

Overeem, N.J., Hamming, P.H., Grant, O.C., Di lorio, D., Tieke, M., Bertolino, M.C., Li, Z., Vos, G., De Vries, R.P., Woods, R.J., Tito, N.B., Boons, G.J.P.H, Van der Vries, E., Huskens, J., ACS Central Science 2020, 6, 2311-2318 


\subsection{Introduction}

Cross-species (zoonotic) transmission of viruses such as influenza $A$ and coronaviruses, is a continuous threat to humans. ${ }^{1,2}$ Yet, the determinants governing the ability of these viruses to switch host species and potentially trigger pandemics, remain poorly understood. ${ }^{3}$ A clearer understanding of the mechanisms underlying virus-host interactions at the molecular level allows for better predictions of the threats of emerging viruses. ${ }^{4,5}$ These insights can also shed light on other unexplained virus phenotypes, such as restricted host ranges and varying degrees of pathogenicity.

The hemagglutinin (HA) of influenza A virus (IAV) initiates infection by binding to sialic acid-terminated glycan receptors on the host cell glycocalyx. Mutations in the receptor-binding domain (RBD) of HA can lead to changes in both affinity and specificity. These changes are associated with influenza zoonoses, ${ }^{6-8}$ antigenic drift, ${ }^{9}$ transmissibility, ${ }^{10,11}$ and pathogenicity. ${ }^{4,12}$ Mutations outside the RBD may influence sialoglycan binding indirectly by adjustment of HA thermostability or virus morphology. ${ }^{13,14}$

While the HA of avian IAV preferentially binds to sialic acids linked via an $\alpha 2,3-$ linkage to a penultimate galactose, those adapted to humans favor the $\alpha 2,6$ linkage. ${ }^{6}$ The impact of RBD mutations on receptor binding is reflected in the orientation of these receptors in their complex with $\mathrm{HA} .{ }^{15}$ With sialic acid in the binding pocket, the remaining glycan residues extend in different topologies based on the $\alpha 2,3$ - or $\alpha 2,6$-linkage and interactions between the glycan and the surface of HA. ${ }^{16}$ In avian-adapted HA subtypes, the $\alpha 2,3$-glycans extend out from the RBD perpendicular to the HA protein. In human-adapted HA subtypes, the $\alpha 2,6$-glycans extend over the HA head domain. ${ }^{17,18}$

Although the monomeric solution affinity $\left(K_{\mathrm{D}}\right)$ of for HA-sialoglycan binding is typically in the millimolar range, intact IAV can bind to cells at picomolar virus concentrations. ${ }^{19-22}$ The binding affinity is dependent on receptor density, ${ }^{19,20}$ but the exact number of HA-sialoglycan contributions remains unclear. Quantitative assessment of influenza interactions with the host cell glycocalyx requires a method that deconvolutes affinity, specificity, density dependence, and structural aspects of influenza-glycan interactions in a representative environment, as well as a physical-mathematical model that translates the 
individual molecular determinants of the affinity and contact area into the overall affinity and specificity. Previously, a numerical model was applied to show that multivalent particles that form weak receptor-ligand interactions bind to areas of varying receptor density in a nonlinear fashion, characterized by a threshold receptor density at which binding becomes efficient. ${ }^{23}$

This chapter shows how intrinsic structural differences in binding of HA to $\alpha 2,3$ and $\alpha 2,6$ receptors transfer through the length and density of sialoglycans at the cell surface into virus avidity and specificity. We show the development of a multivalent affinity platform to probe these individual contributions in an environment that approximates the molecular architecture of the cell membrane. We accomplished this by creating sialoglycan receptor density gradients on supported lipid bilayers to obtain virus threshold receptor densities. We combine information from multiple platforms to study the combined effects of receptor type, density, and length on the virus binding. We have developed an analytical model to quantitatively describe binding profiles of IAV to assess multivalent binding at the threshold density. Finally, the molecular mechanism is further explored by molecular dynamics simulations to provide detailed insights into structural aspects of the binding process.

\subsection{Results and discussion}

\subsubsection{Multivalent affinity profiling}

To study receptor density dependence of IAV binding we developed multivalent affinity profiling (MAP), a method based on the colocalization of fluorescently labelled viruses and receptors on broad-range and continuous surface gradients. We applied this method to study the binding of IAV as a function of receptor density using glycans with different lengths and linkages. To mimic the presentation of glycans on a cell surface we use biotinylated glycans immobilized by streptavidin (SAv) on a supported lipid bilayer (SLB) (Figure 4.1a). The glycans consist of sialic acid $\alpha 2,3$ - or $\alpha 2,6$-linked to one, two, or three $\mathrm{N}$-acetyl lactosamine repeats $\left(2,3-\right.$ or $2,6-\mathrm{S}(\mathrm{LN})_{\mathrm{n}}$, Figure $\left.4.1 \mathrm{~b}\right)$. They are presented in a density gradient to visualize the threshold receptor density, above which viruses bind with maximum coverage, and with negligible coverage below (Figure 4.1c)..$^{23,24}$ This threshold receptor density is the key indicator of virus binding specificity in the MAP platform. 

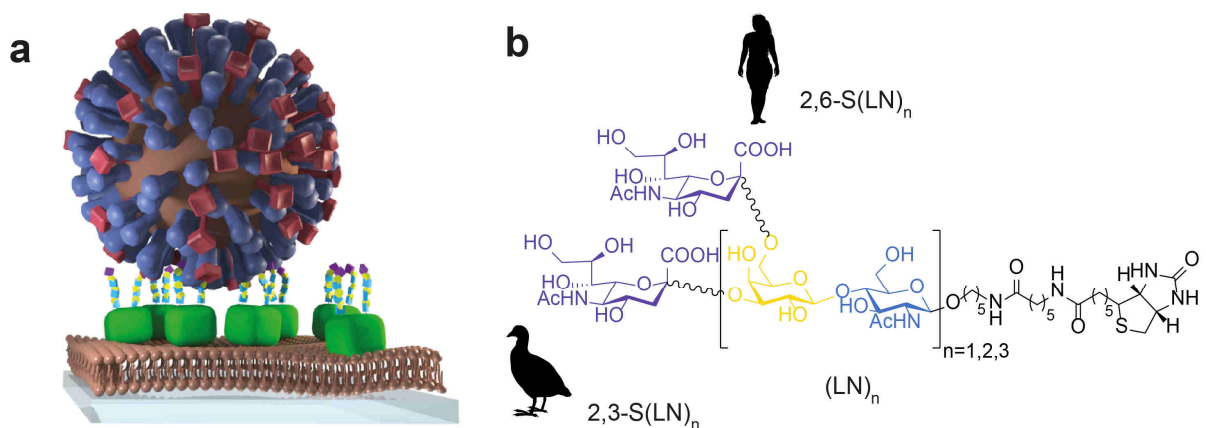

C
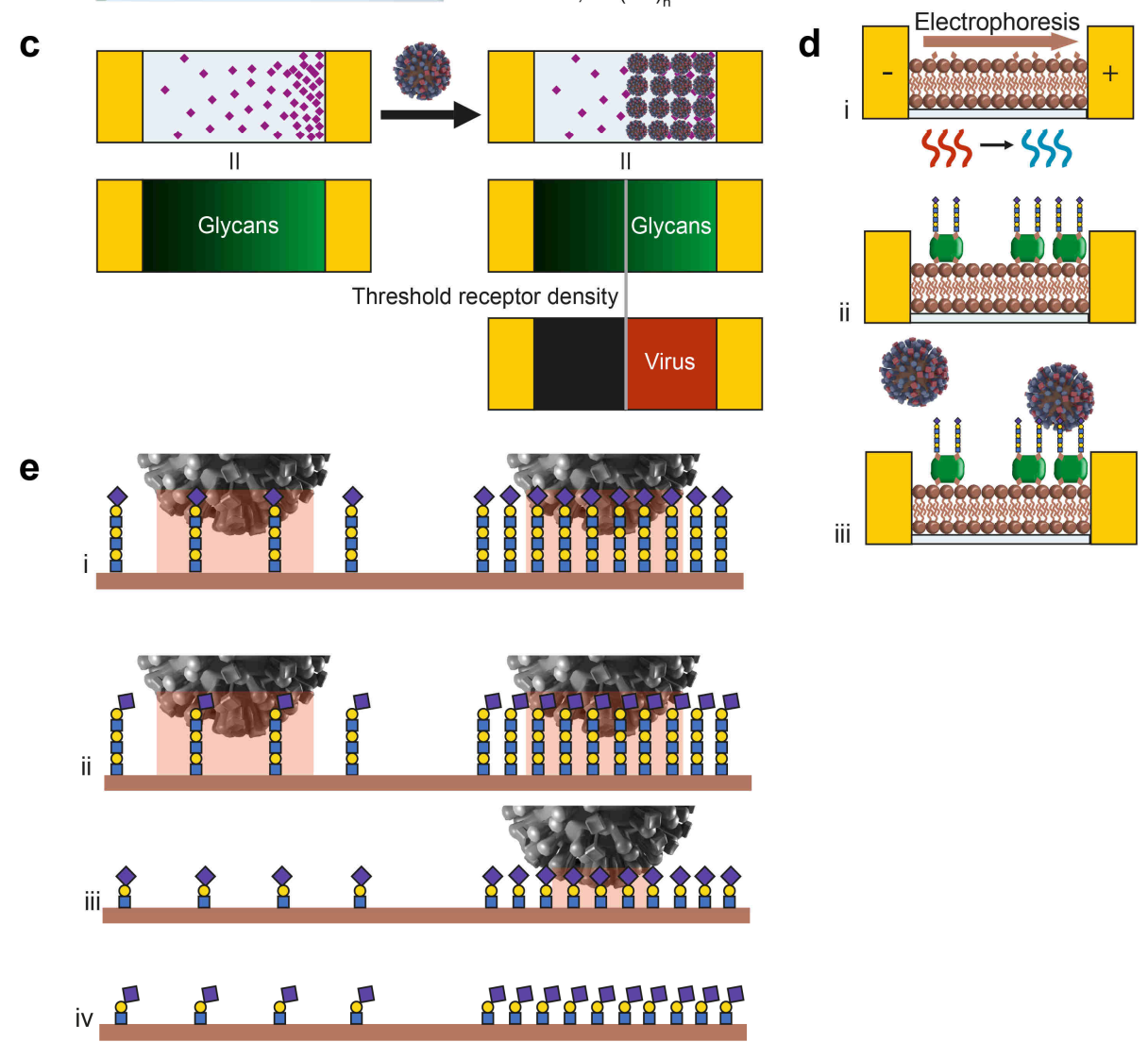

Figure 4.1| The MAP chip is a platform to quantitate IAV binding to a host cellmimicking surface. a) Cartoon showing how glycans immobilized by streptavidin onto supported lipid bilayers (SLBs) were used to mimic the host cell architecture (not to scale). b) Structure of the biotinylated glycans. 2,3-S(LN $)_{n}$ are avian-type receptors and 2,6-S(LN) $)_{n}$ human-type. $(\mathrm{LN})_{2}$ has no sialic acid and was used to control receptor density on QCM and BLI platforms. c) Schematic representation of the MAP platform, where viruses only bind above the threshold receptor density for a particular virus-sialoglycan 
combination. d) Methodology to create the gradient platform and to study virus binding on receptor gradients. Electrophoretic gradients of biotinylated lipids are formed in SLBs in a microfluidic device at elevated temperature and subsequently cooled down to lock the gradients (i). The biotin gradient is modified with fluorescently labelled streptavidin (SAv) and biotinylated sialoglycans (ii). The fluorescently labelled influenza virus is passed over the SLBs with the sialoglycans acting as receptors (iii). e) Cartoons showing how length and linkage of the sialoglycans can affect virus binding in the case of PR8. The contact area is indicated in peach. If both receptor length and linkage (2,3 or 2,6$)$ are favorable, the virus binds at high and low receptor density (i). If linkage is unfavorable (ii), PR8 may still bind at high and low densities. Other influenza viruses may show a stronger preference for one receptor type and bind only at high densities. If length is unfavorable (iii), the virus binds only at high receptor density. If both are unfavorable, the virus may not bind at all (iv).

The MAP chip gradients are formed in a microfluidic device that we developed for this purpose. ${ }^{25,26}$ First, we comixed a charged biotinylated lipid and a zwitterionic base lipid (MPPC) to form the SLB and subsequently subjected the SLB to an electrical field at elevated temperature, yielding stable surface-biotin gradients at room temperature (Figure 4.1d). We then functionalized the gradients of biotinylated lipids with fluorescently labelled $S A v$ and $S(L N)_{n}$, and subsequently passed fluorescently labelled IAV over these receptor gradients and mapped the colocalization of virus and receptors to determine the threshold receptor density. This allows us to study the combined effect of the glycan length and 2,3- or 2,6-linkage on the binding of influenza as a function of the receptor density (Figure $4.1 \mathrm{e}$ ).

\subsubsection{Threshold density dependence on receptor type and length}

To study the density-dependent binding of IAV on different glycan types, we used UV-inactivated influenza A/Puerto Rico/8/34 (PR8) Mount Sinai strain with fluorescent label. The colocalization of this virus with $S(L N)_{n}$ receptors on greenlabelled streptavidin was imaged with fluorescence microscopy (Figure 4.2a and $2 b$ ). While the receptors formed a gradient with an exponential curve, the fluorescent signal of the virus showed the characteristic sigmoidal profile (Figure 4.2c). Because the average receptor density of the gradient is known, the sigmoidal binding profile of the virus can be related to the absolute receptor densities of 2,3- and 2,6-S(LN) (Figure 4.2d). Virus binding data from multiple pairs of micrographs were combined to afford these binding profiles (Figure 4.S1 and 4.S2). 


\section{Chapter 4}
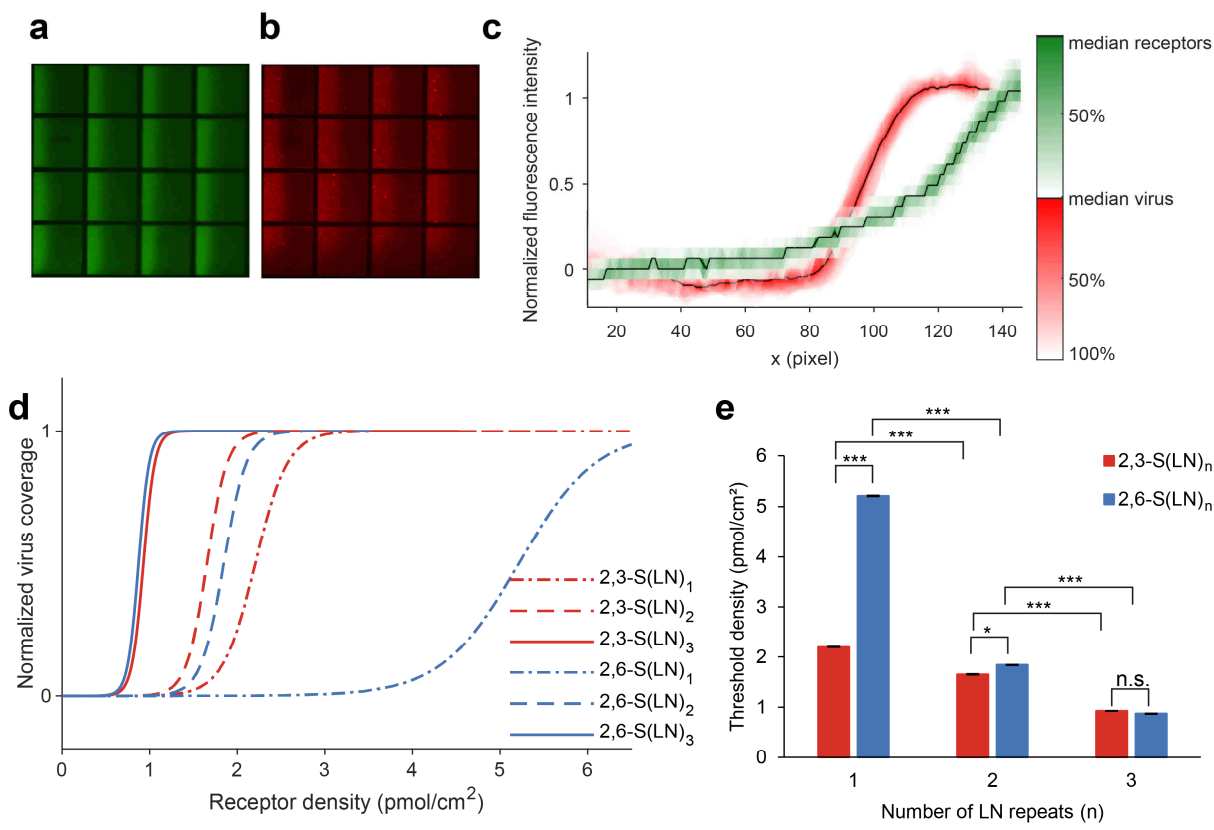

Figure 4.2 Multivalent affinity measurements using different glycan receptors. a) Fluorescence micrograph of receptor gradients with labelled SAv. b) Fluorescence micrograph of adsorbed labelled virus, binding selectively at high receptor densities. c) Fluorescence intensity profiles of labelled SAv (green) and virus (red) as function of distance along the horizontal direction. The black lines show the median along the vertical direction. The variance is given by the red and green contours which indicate the percentage of data points between each contour line and the median. d) Multivalent affinity profiles of influenza PR8 virus as a function of receptor density. The data to which the curves were fitted is shown in Figure 4.S1. e) Threshold receptor densities for $S(L N)$ n. Error bars show the $95 \%$ confidence interval of the fit. n.s.: not significant, $*: p<0.05, * * *: p<0.001$. 

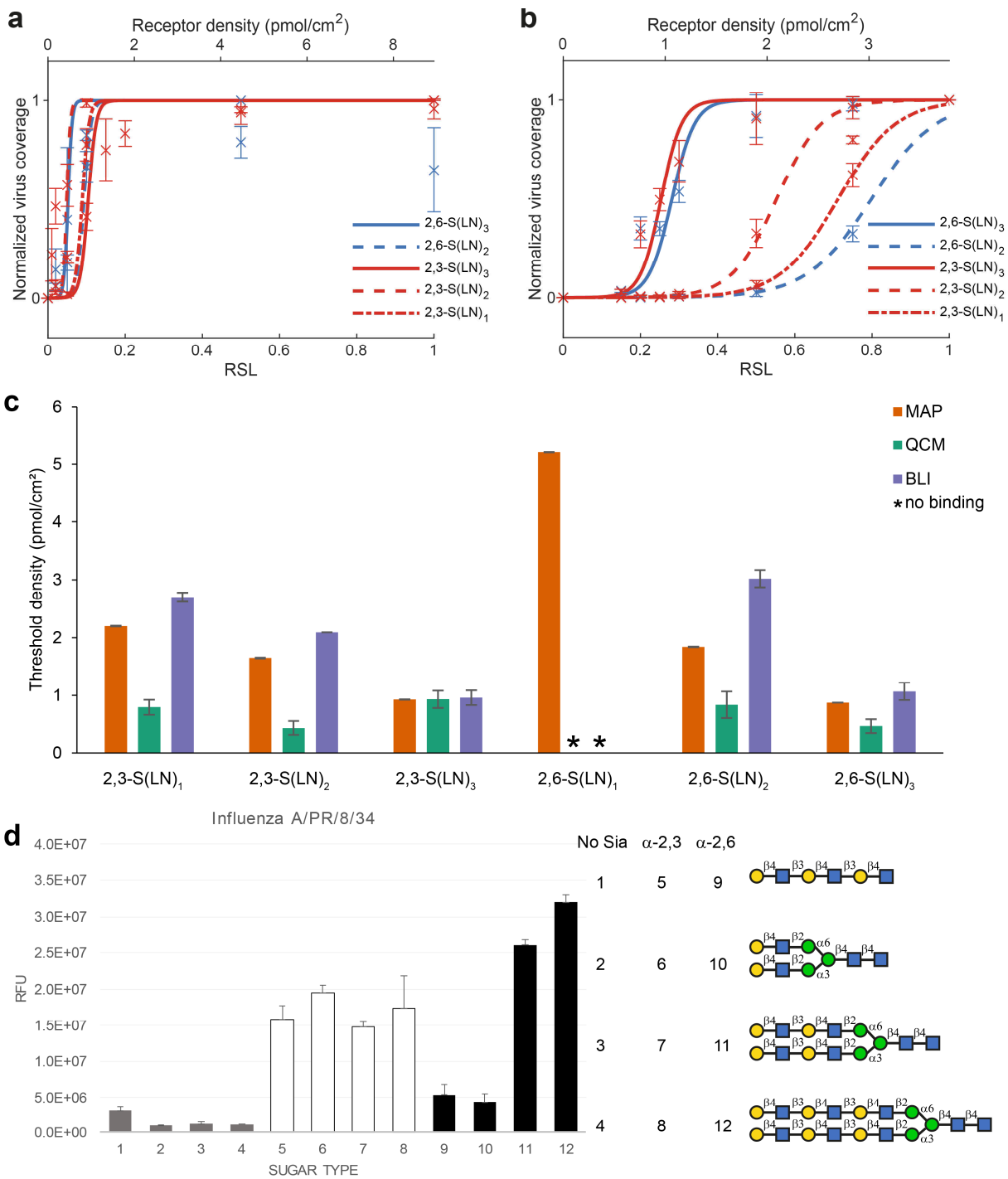

Figure 4.3| Binding profiles of influenza virus on different platforms. a) Binding profiles as function of relative sugar loading (RSL) and receptor density in QCM. b) Binding profiles as function of RSL and receptor density in BLI. c) Overview of threshold receptor densities for MAP, QCM, and BLI. The receptor densities have not been externally calibrated and may be up to twofold higher for MAP and BLI, and may be somewhat lower for QCM. A different calculation should affect all threshold densities for the same technique similarly. d) Fluorescence intensities of PR8 binding on a glycan microarray in relative fluorescence units (RFU). Binding to a linear 2,6-S(LN) $(\# 9)$ and to a branched 2,6-S(LN) 1 glycan (\#10) are both much lower than either their 2,3-linked 
counterparts (\#5 and 6) or branched 2,6-S(LN) $)_{2}$ and 2,6-S(LN) $)_{3}(\# 11$ and 12 ), suggesting that PR8 is also more sensitive to the branching of 2,6- than of 2,3-linked glycans.

We observe for PR8 that the threshold receptor densities for all glycans lie within the range of $0.9-5.2 \mathrm{pmol} / \mathrm{cm}^{2}$. The longest 2,3- and 2,6-S(LN) $)_{3}$ glycan receptors display the lowest binding thresholds, which are the same for both glycans (Figure 4.2e). The threshold density becomes higher with shorter glycans, but the effect is more profound for $2,6-S(L N)_{n}$ than for 2,3-S(LN) . Analyses with quartz crystal microbalance (QCM, Figure 4.3a) and biolayer interferometry (BLI, Figure 4.3b), show approximately the same threshold density values, but the binding for $2,6-\mathrm{S}(\mathrm{LN})_{1}$ was too low to detect using these techniques (Figure 4.3c).

Similarly, we compared the data with the binding of PR8 on a traditional glycan array (Figure $4.3 \mathrm{~d}$ ). Linear $\mathrm{S}(\mathrm{LN})_{3}$ and branched glycans of different lengths, each with 2,3-, 2,6- or without sialic acid, were immobilized on a microarray and incubated with labelled PR8. The relative fluorescence intensity of bound PR8 was measured. As observed for MAP, the microarray data show that PR8 can bind to both 2,3- and 2,6-linkages, and the binding to shorter glycans is lower, in particular for 2,6-linked glycans. Interestingly, PR8 is also more sensitive to the branching of 2,6- than of 2,3-linked glycans.

\subsubsection{A theoretical model to describe the multivalent binding of influenza virus}

To understand how glycan type and length affect the position of the threshold receptor density, we developed a theoretical binding model, based on the statistical thermodynamics of multivalent adsorption. ${ }^{23,27}$ In Appendix 4.6.1, we derive a theoretical expression (Equation 4.1) for the multivalent equilibrium binding constant $K_{\mathrm{av}}$ of virus particles on a receptor surface and the average number of possible simultaneous virus-glycan receptor interactions $\tilde{N}$ (Equation 4.2). The resulting expressions depends on several fundamental properties of the virus, glycans, and surface receptor chemistry (shown in Figure 4.4a and 4.4b):

$$
\begin{aligned}
& K_{\text {av }}=N_{\mathrm{A}} V_{\text {ex }}\left(1+\frac{K_{\text {i,eff }}}{N_{\mathrm{A}} V_{\text {explore }}}\right)^{\tilde{\mathrm{N}}} \\
& \tilde{\mathrm{N}}=A_{\text {contact }} \cdot \min \left(\sigma_{\mathrm{R}}, \sigma_{\mathrm{L}}\right)
\end{aligned}
$$


a
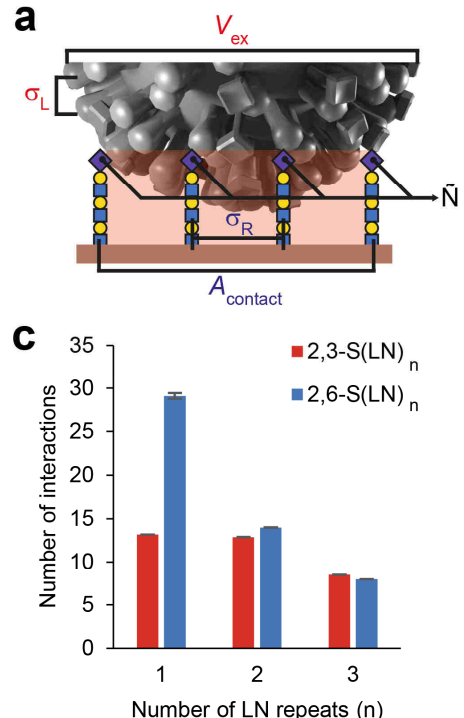

b
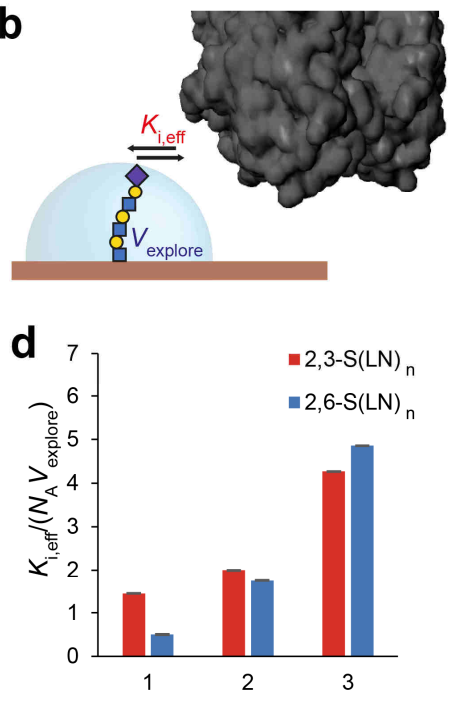

Number of LN repeats (n)

Figure 4.4| Theoretical model for multivalent binding of influenza. a) Cartoon of a virus bound to a glycan-coated surface with model parameters indicated. $\sigma_{L}$ is the density of RBDs on the virus. $V_{\text {ex }}$ is the excluded volume of a bound virus, which is a function of its diameter. $\tilde{N}$ is the average number of HA-glycan interactions that can form simultaneously. $\sigma_{R}$ is the density of receptors on the surface. $A_{\text {contact }}$ is the receptor surface area that can be reached by RBDs on the virus. b) Zoom-in of a single HA-glycan interaction. $K_{\mathrm{i} \text {,eff }}$ is the monovalent receptor-ligand equilibrium of a surface-bound glycan and virus-bound RBD. Vexplore is the volume accessible to a glycan. c) Values of $\tilde{N}_{\text {threshold }}$ for different glycan types and lengths, calculated from the threshold receptor density and maximum length of each glycan. d) Fitted values of $K_{\mathrm{i}, \text { eff }} / N_{\mathrm{A}} V_{\text {explore, }}$ the contribution of individual interactions to the avidity.

The parameters in red are virus characteristics: the excluded volume $V_{\text {ex }}$ of a virus particle, the effective equilibrium constant of an individual HA-glycan interaction $K_{\mathrm{i} \text {,eff }}$ (the inverse of $K_{\mathrm{D}}$ ) and the RBD density on the virus surface $\sigma_{\mathrm{L}}$. The parameters in blue are surface characteristics: the receptor density $\sigma_{R}$, the volume accessible to a glycan $V_{\text {explore, }}$ and the surface area that can be reached by RBDs when the virus is bound $A_{\text {contact. }}$ The quantity $\left(1 / N_{\mathrm{A}} V_{\text {explore }}\right)$, where $N_{\mathrm{A}}$ is Avogadro's number, is the "effective molarity" of glycan receptors on the surface, from the perspective of a single HA monomer on a bound virus. The effective equilibrium constant $K_{\mathrm{i} \text {,eff }}$ can be higher than the equilibrium constant in solution due to mass-transfer effects associated with rebinding to the 
surface, ${ }^{28}$ or lower due to additional free energy costs such as from steric repulsion between $\mathrm{HA}$ and the surface and any configurational entropy cost for forming the bond. ${ }^{29,30}$ Although no significant nonspecific binding was observed in a binding study without glycans in QCM-D (Figure 4.S3a), the possible effect of a nonspecific free energy contribution on the virus binding profiles is also discussed in Appendix 4.6.1.

We applied the theoretical model to fit the binding profiles of IAV (Figure 4.2d) and determine the number of interactions needed at the threshold and $K_{\mathrm{i} \text {,eff }}$ (Appendix 4.6.1). Molecular dynamics (MD) simulations were used to determine the minimum and maximum length of each glycan and to derive $A_{\text {contact }}$ and $V_{\text {explore }}$ (Figure 4.S2). The number of interactions at the threshold $\tilde{\mathrm{N}}_{\text {threshold }}$ is calculated using Equation. 2. Because $\sigma_{\mathrm{L}}$ is on average 3.8 $\mathrm{pmol} / \mathrm{cm}^{2},{ }^{31}$ and can locally be as high as $7.7 \mathrm{pmol} / \mathrm{cm}^{2}$ for close-packed $\mathrm{HA},{ }^{32}$ $\sigma_{R}$ is generally lower than $\sigma_{L}$ at the threshold density (Figure 4.2e). This applies even to 2,6-S(LN) $)_{1}$ if we assume that viruses that bound at the threshold density used their areas with the highest HA density. $\tilde{\mathrm{N}}_{\text {threshold }}$ is therefore the product of the threshold receptor density and $A_{\text {contact }}$ (Figure 4.4c). $\tilde{\mathrm{N}}_{\text {threshold }}$ is approximately 8 for $\mathrm{S}(\mathrm{LN})_{3}$ and up to 29 for $2,6-\mathrm{S}(\mathrm{LN})_{1}$ as shorter glycans contribute less to the overall avidity than longer glycans (Figure $4.4 \mathrm{~d}$ ), thus requiring a higher number of interactions to bind.

\subsubsection{Molecular modelling of HA-glycan binding}

To understand the influence of glycan length, structure and presentation on the virus binding, $\mathrm{MD}$ simulations were employed to explore the possible shapes $\mathrm{S}(\mathrm{LN})_{\mathrm{n}}$ glycan-linker-biotin molecules can adopt when bound to the SAv tetramer. To take into account the orientational constraints of the HA and SAv molecules that arise from their immobilization on the virus and array surfaces, respectively, the angle between the center lines of the HA and SAv was determined (Figure 4.5a). Based on a simple geometric model (Figure 4.5c) for a spherical virus interacting with glycans projecting from a flat SAv surface, the allowable angular range for the glycan relative to the surface depends on the glycan length and the virion radius. The MD data were used to provide estimates of the average glycan length for each type of glycan bound to SAv. With these values, the allowable angles were determined (Figure 4.5b; Appendix 4.6.2). 
The percentage of shapes for each SAv-immobilized glycan type that could allow HA binding were determined (Figure 4.5b). In general, as the glycan becomes shorter, it displays fewer shapes compatible with HA binding, particularly in the case of 2,6-S(LN) 1 , which correlates with the observed avidity data (Figure 4.2e). The decrease in shapes compatible with HA binding corresponds to the decrease in conformational entropy of the glycans but ignores interactions between the HA head and LN repeats. ${ }^{17}$ For each glycan, the total number of orientations with allowable angles correlates well with the observed dependence on receptor densities (Figure 4.2); the shorter glycans, especially $2,6-\mathrm{S}(\mathrm{LN})_{1}$, have fewer acceptable glycan poses and therefore require a higher receptor density to bind the virus.

Because the modes of binding that are accessible are unequally affected by glycan length, we investigated (Appendix 4.6.2) the conditions under which: A) two glycans on different SAvs could bind to different RBDs in the same HA trimer ( $2 \mathrm{SAv}-1 \mathrm{HA}$ ), B) two glycans on the same SAv could bind to two different $H A$ trimers (1 SAv - $2 \mathrm{HA}$ ), or C) two glycans on the same SAv could bind to the same HA trimer ( $1 \mathrm{SAv}-1 \mathrm{HA}$ ). Both the $2 \mathrm{SAv}-1 \mathrm{HA}$ mode and the $1 \mathrm{SAv}-2$ $\mathrm{HA}$ mode are more accessible for $2,3-\mathrm{S}(\mathrm{LN})_{\mathrm{n}}$ glycans than for $2,6-\mathrm{S}(\mathrm{LN})_{\mathrm{n}}$ and are more strongly affected by glycan length for $2,6-\mathrm{S}(\mathrm{LN})_{\mathrm{n}}$. The bidentate $1 \mathrm{SAv}-1$ $\mathrm{HA}$ mode was only accessible to $2,6-\mathrm{S}(\mathrm{LN})_{3}$ and not to shorter or 2,3-linked glycans, which is consistent with the findings of Peng et al..$^{33}$ and Nemanichvili et al. ${ }^{18}$ for branched glycans. 


\section{Chapter 4}

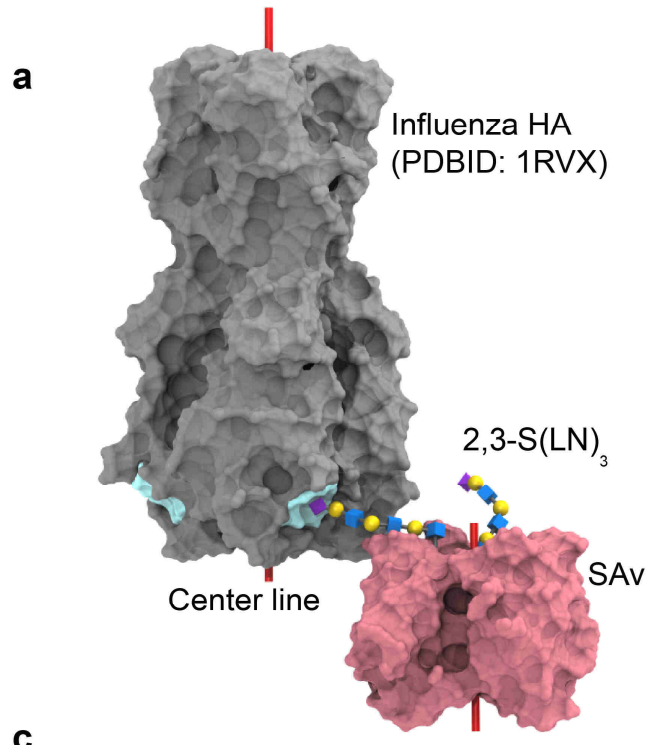

b

C
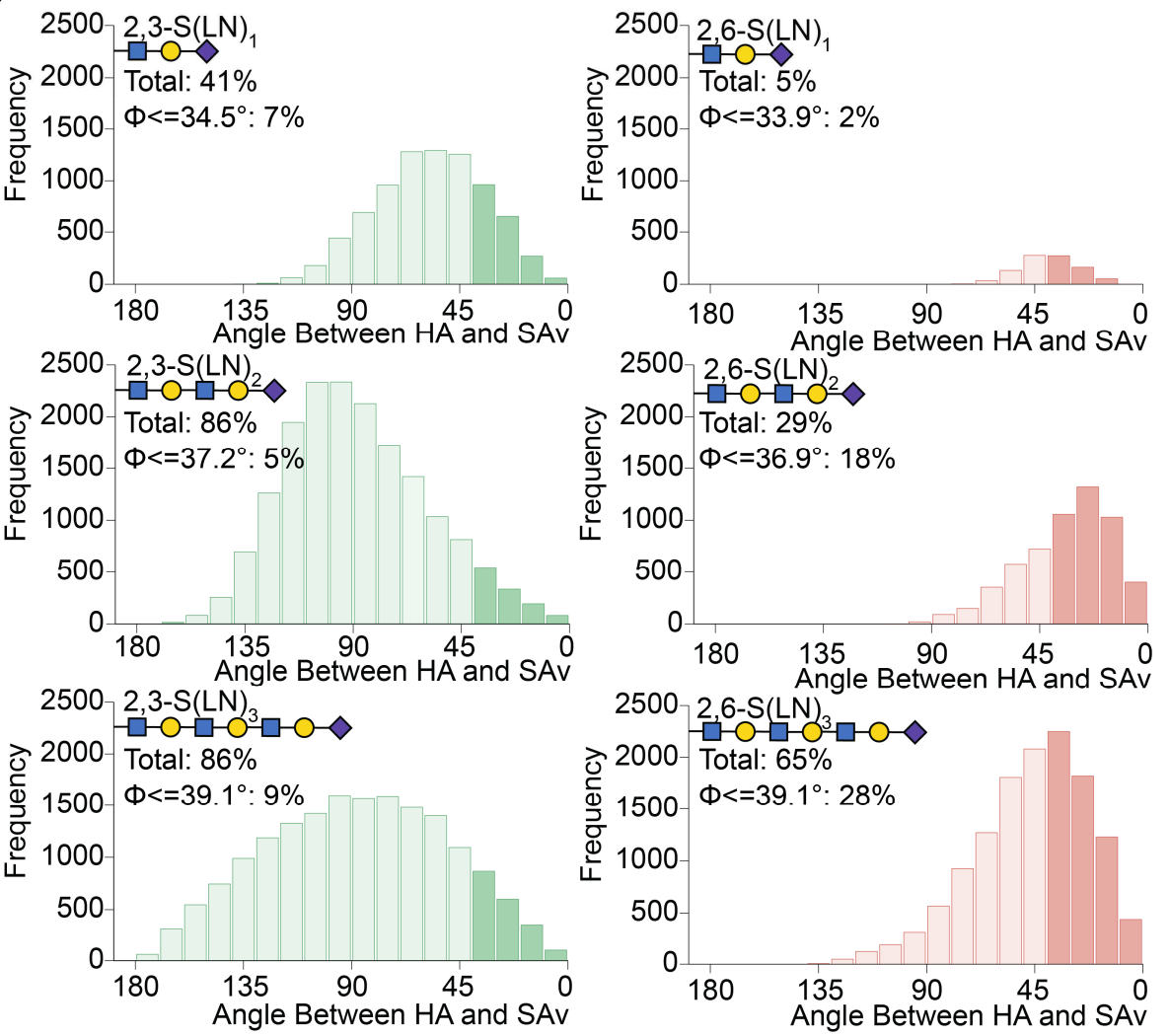
4Figure 4.5| Glycan shapes and HA-SAv angles that lead to binding in molecular dynamics. a) The crystal structure of influenza HA in complex with 2,3-S(LN) (gray surface, binding sites in cyan) superimposed onto the biotinylated 2,3-S(LN) 3 bound by SAv via the penultimate Gal residue of the glycan (shown as 3D-SNFG). This superimposition was repeated for each of the four biotinylated glycans in each glycan system, and for each snapshot taken from the MD simulations. Any structures with atomic overlaps between the HA and SAv were removed from consideration. The angle between the two center lines of the HA and SAv was measured $\left(180^{\circ}\right.$ for the structure shown here). b) Histogram plots of the frequency of shapes that did not result in atomic overlap between $\mathrm{HA}$ and $\mathrm{SAv}$ as binned every $10^{\circ}$ of the angle between the center lines of SAv and HA. The total percentage of glycan shapes without atomic overlap and the percentage of shapes accessible if the rotations of $\mathrm{HA}$ and SAv are restricted are shown under each glycan structure ( $\mathrm{N}=20,000$ for each system). c) Range of acceptable angles $(\phi)$ between the $\mathrm{HA}$ axis and the normal to the flat surface as a function of virion radius (R) and glycan length (L).

\subsubsection{Discussion}

Glycan structure and presentation have a profound influence on both the monovalent and multivalent surface affinities. The values for $K_{\mathrm{i} \text {,eff }}$ that we found for $\mathrm{S}(\mathrm{LN})_{3}$ (Figure 4.S2f) are comparable to the solution equilibrium constants that were measured for X-31, which has HA and NA of Aichi/2/68 (H3N2) and other genes from PR8, and has highly similar binding profiles in BLI. ${ }^{19,34}$ For $\mathrm{S}(\mathrm{LN})_{1}, K_{\mathrm{i}, \text { eff }}$ is an order of magnitude lower, which is consistent with the fewer number of shapes of the immobilized $S(L N)_{1}$ glycan that are able to bind to the $H A$. This is consistent with the increased entropic cost of forming an $S(L N)_{1}-R B D$ interaction that is suggested by MD (Figure 4.5b). In addition, the entropy of the multivalent virus-surface interaction is affected by differences in the availability of multivalent binding modes (Appendix 4.6.2). The $1 \mathrm{SAv}-2 \mathrm{HA}$ and $2 \mathrm{SAv}-1 \mathrm{HA}$ modes are both more accessible to 2,3- than to 2,6-S(LN) $)_{n}$. The equally high avidity observed for $2,6-\mathrm{S}(\mathrm{LN})_{3}$ could therefore be a result of its unique ability to form a bidentate interaction between two SAv-bound glycans and a single HA. Because all modes are less available to the shorter 2,6-S(LN) and $2,6-\mathrm{S}(\mathrm{LN})_{2}$, their avidity is lower.

Through the length and density of glycans, their effects on virus binding translate into different multivalent affinities and selectivities. This is especially evident for PR8, which binds readily to both $\alpha 2,3$ - and $\alpha 2,6$-linked sialoglycans, but likely applies also to other IAVs possessing clear specificity. The type, length 
and density dependence of these complex virus-receptor interactions may explain why some IAVs infect certain cell types and others do not. For example, duck intestinal mucosa is rich in $\alpha 2,3$-sialosides whereas human upper airway tissue expresses high levels of $\alpha 2,6$-sialosides. ${ }^{35}$ The IAVs circulating in humans are of avian origin and acquired the ability to use $\alpha 2,6$-linked sialosides. It is becoming increasingly clear that the glycocalyx varies considerably between (host) species, ${ }^{6,36}$ and between different cell types. ${ }^{37,38}$ Not only differences in $\alpha 2,3$ - or $\alpha 2,6$-linkage of sialoglycans, but also their branching, the length of their branches, and the density of sialoglycans determine to which cells IAV binds. Although glycomic studies of human lung tissue have shown the presence of sialosides presented on extended LN moieties, the density of functional receptors is not known and glycan arrays that present a variety of $\alpha 2,3-$ and $\alpha 2,6$-linked sialoglycans of varied length and branching are unable to predict infection of human respiratory tissue. ${ }^{38}$

The total density of sialic acid in the glycocalyx of cells is typically 10 -fold higher than the threshold density on our sensor. ${ }^{39,40}$ However, as the glycocalyx presents a dense brush that extends approximately $1 \mu \mathrm{m}$ from the cell surface, ${ }^{41}$ only a small fraction of these moieties can be involved in the initial binding of a virus so that only the density variations near the outer surface of the glycocalyx may be involved in host cell recognition. That the accessibility of sialoglycans is more important than their number is supported by the observations on human alveolar epithelial cells that IAVs associate more strongly with the mucin MUC1 than with the much larger MUC $16^{42}$ as well as in synthetic systems where IAVs responded to density variations of sialic acid-modified polymers in a mucus-like brush where each polymer displayed on average 90 sialic acid groups. ${ }^{43}$

For weaker-binding IAVs than PR8, where the threshold receptor density would be higher than their density of RBDs, the 3D presentation and flexibility of receptors may play a major role in their specificity by forming sufficient interactions only when the glycocalyx shapes itself to the virus. Most human IAVs bind less strongly to $\alpha 2,3$-linked glycans than PR8 but more strongly to $\alpha 2,6$-linked glycans of the same length, whereas the opposite is true for most avian IAVs, and some such as Anhui/1/2013 (H7N9) bind less strongly to glycans of both linkages. ${ }^{19,20,34,43,44}$ It is therefore likely that variations in both the density and structure of sialoglycans in the glycocalyx play a role in the host cell specificity of IAV. The preference of IAV to bind one cell type over another was 
further associated with increased airborne transmissibility ${ }^{11}$ and $\mathrm{A} / \mathrm{H} 5 \mathrm{~N} 1$ virus pathogenicity. ${ }^{37}$

\subsection{Conclusions}

The MAP chip and supporting model offer a method to quantitatively characterize the multivalent binding of any virus that binds multivalently to cellular glycan receptors. This study shows that IAV needs a specific minimum number of RBD-glycan pairs to form. Not only the structure of the virus but also that of the glycans determines the density at which this number of interactions is achieved. This way, intrinsic structural (and energetic) differences of an $\alpha 2,3-$ or $\alpha 2,6$-linked glycan in the RBD are translated into different multivalent binding profiles. MAP may allow studying not only how individual RBD receptor mutations influence the $K_{\mathrm{i}}$ but also how changes in virus morphology affect $K_{\mathrm{av}}$. We expect that it is through multivalency that such mutations are associated with virus pathogenicity and transmissibility. MAP may therefore be used in concert with quantitative glycomics to further elucidate the connection between the multivalency of viruses and these phenotypes in order to predict the tropism, transmissibility and pathogenicity of novel zoonotic viruses.

\subsection{Acknowledgements}

Erik Hamming is thanked for his role in the image analysis and the fitting of the analytical model to experimental data. Daniele Di lorio and Candelaria Bertolino are thanked for the QCM studies. Malte Tieke and Erhard van der Vries are thanked for the production and characterization of the viruses and for the BLI studies. Zeshi Li, Gaël Vos, and Geert-Jan Boons are thanked for the synthesis of the glycans. Robert de Vries is thanked for the glycan array studies. Oliver Grant and Robert Woods are thanked for the MD simulations. Nicholas Tito is thanked for the analytical model. Wouter Vijselaar is thanked for the cleanroom fabrication of the gradient chips.

\subsection{Experimental}

\subsubsection{Biotinylated glycan preparation}

Glycans were prepared based on already reported procedures. ${ }^{45,46}$ Biotinylated LacNAc was synthesized chemically and used as starting material for one-pot 
enzymatic extension. Briefly, the starting disaccharide was dissolved in $100 \mathrm{mM}$ MOPS buffer $\mathrm{pH} 7.2$ to a $10 \mathrm{mM}$ working concentration. UDP-GIcNAc $(20 \mathrm{mM}$, 2 eq.), $\mathrm{MnCl}_{2}(10 \mathrm{mM})$ and calf-intestine alkaline phosphatase $(10 \mathrm{U} / \mathrm{ml})$ were added. The GIcNAc transferase beta3GnT2-GFP was then added at the working concentration of $0.1 \mathrm{mg} / \mathrm{ml}$. The reaction solution was incubated at $37^{\circ} \mathrm{C}$ for 24 $\mathrm{h}$ under mild agitation. The solution was heated to $100^{\circ} \mathrm{C}$ for $10 \mathrm{~min}$ to denature the enzymes if no starting material was observed by ESI-MS. Otherwise another $0.05 \mathrm{mg} / \mathrm{ml}$ glycosyltransferase was added, and the reaction was incubated for another $24 \mathrm{~h}$. After cooling to room temperature, UDP-Gal (2 eq.) and the galactosyltransferase B4GalT1 was added at the working concentration of 0.1 $\mathrm{mg} / \mathrm{ml}$. The solution was incubated overnight at $37^{\circ} \mathrm{C}$ to allow galactosylation to go to completion (monitored by ESI-MS). Enzyme denaturization was performed as mentioned for beta3GnT2. This tandem enzymatic treatment (beta3GnT2-B4GalT1) was repeated to afford triLacNAc structure without any intermediate column chromatography-based purification. In between each reaction, the solution was replenished with fresh UDP-sugar donors (1 eq.), $\mathrm{MnCl}_{2}$ and alkaline phosphatase. When the precipitate (generated during beta3GnT2 treatment) became over half of the volume, the mixture was spun down and the supernatant was transferred into a new vial. The precipitate was washed with $1 / 5$ initial reaction volume of buffer, spun down and the supernatant was combined. The solution containing triLacNAc-biotin was freeze-dried and purified with biogel-p2 to give the pure product in $\sim 60 \%$ yield.

The triLacNAc-biotin was then sialylated in $\alpha 2,6$-linkage with ST6Gal1 and $\alpha 2,3$ with ST3Gal4. The triLacNac-biotin was dissolved in $100 \mathrm{mM}$ TRIS buffer $\mathrm{pH} 8$ to a $10 \mathrm{mM}$ working concentration. CMP-Neu5Ac ( $15 \mathrm{mM}, 1.5$ eq.), bovine serum albumin $(0.1 \mathrm{mg} / \mathrm{ml})$, calf-intestine alkaline phosphatase $(10 \mathrm{U} / \mathrm{ml})$ and the appropriate sialyltransferase $(0.1 \mathrm{mg} / \mathrm{ml})$ were added. The solution was incubated overnight at $37^{\circ} \mathrm{C}$ to allow sialylation to go to completion (monitored by ESI-MS).The resulting heptasaccharide was purified with biogel-p2 and fractions containing carbohydrate were pooled and freeze-dried to give the pure form as a white crystalline powder for subsequent experiments.

\subsubsection{Virus stock preparation}

Influenza A/Puerto Rico/8/34 virus (Mt. Sinai strain) stocks were prepared by propagating the virus in 10-days-old embryonated chicken eggs (GD Animal 
health, Deventer, The Netherlands) at $33^{\circ} \mathrm{C}$. Allantoic fluids were harvested after 48-72 $\mathrm{h}$ and cleared from debris by centrifugation at 3,000 rpm for $10 \mathrm{~min}$ at $4{ }^{\circ} \mathrm{C}$. Subsequently, the viruses were pelleted by centrifugation at 7,000 rpm for $18 \mathrm{~h}$ at $4{ }^{\circ} \mathrm{C}$ and resuspended in PBS (pH 7.4) (Lonza). Viruses were then purified further by loading of the virus sample on a discontinuous sucrose gradient $(10-50 \% \mathrm{w} / \mathrm{v})$ and centrifugation for $45 \mathrm{~min}$ at $25,000 \mathrm{rpm}$ using a SW41 swing-out rotor. The virus-containing sucrose layer was finally harvested and dialyzed (Slide-A-Lyzer, Thermo Scientific) for $48 \mathrm{~h}$ at $4{ }^{\circ} \mathrm{C}$ to remove the remaining sucrose. Finally, the virus particle count and size distribution were determined using a NS300 nanoparticle tracking analyzer (Malvern, Nanosight), and diluted to a final stock concentration of $1 \times 10^{11}$ particles $/ \mathrm{ml}$, UV-inactivated $\left(50 \mathrm{~mJ} / \mathrm{cm}^{2}\right.$, at a wavelength of $\left.365 \mathrm{~nm}\right)$ and aliquots were frozen at $-80^{\circ} \mathrm{C}$. Inactivation was confirmed by growing of these viruses on Madin-Darby canine kidney (MDCK) cells. The receptor binding capacity of the viruses was confirmed with a hemagglutination assay that was performed as described elsewhere ${ }^{47}$ to obtain a titer of 2048.

\subsubsection{Virus labelling}

Influenza viruses were fluorescently labelled using the lipophilic dye octadecyl rhodamine B (R18; Thermo Scientific), which binds the virus membrane with the fluorophore at the aqueous interface. R18 dye was mixed with inactivated virus stocks to a final concentration of $80 \mathrm{nM}$ and incubated in the dark for $2 \mathrm{~h}$ on ice. Free dye was subsequently separated from the R18-labelled viruses by adding $25 \mu \mathrm{l}$ Capto core 700 virus purification beads (GE Healthcare) per $1 \mathrm{ml}$ of virus and incubating by rotating the mixture for $30 \mathrm{~min}$ at $4^{\circ} \mathrm{C}$. Finally, the beads were cleared from the virus-containing supernatant by pelleting of the beads by centrifugation at $1,200 \mathrm{rpm}$ for $10 \mathrm{~min}$ in a table centrifuge at $4^{\circ} \mathrm{C}$.

\subsubsection{Chip fabrication}

Flow cells were fabricated according to the procedure described earlier in Chapter 3. A bilayer lift-off recipe was used for fabricating Au electrodes on Mempax glass wafers (Schott). First, LOR 5A (MicroChem) was spin-coated, after which normal lithography was performed on top with Olin OiR 907-17 photoresist (FujiFilm) to create a bilayer resist stack. Electrode patterns were made by exposing the photoresist through a patterned photomask and developing in Olin OPD 4262 (Fujifilm). The develop step washed away the 
exposed photoresist, and etching through the LOR $5 \mathrm{~A}$ layer created an undercut. Then, $5 \mathrm{~nm}$ Ti and $95 \mathrm{~nm}$ Au were deposited via e-beam evaporation (BAK 600, Balzers). The bilayer resist was then removed by sonication in acetone ( $20 \mathrm{~min}$ ) and isopropanol (10 $\mathrm{min}$ ) followed by $5 \mathrm{~min}$ immersion in OPD 4262, serving as a sacrificial layer to leave patterned Au electrodes on Mempax glass. To fabricate the $\mathrm{Cr}$ corrals ( $10 \mathrm{~nm}$ thick) in between the Au electrodes, the same procedure was performed a second time, but in this case following alignment with respect to the Au electrodes.

\subsubsection{PDMS flow channel}

Silicon flow channel masters were produced by standard photolithography steps and deep reactive ion etching. The polydimethylsiloxane (PDMS) flow channels were prepared from a degassed mixture of 10:1 Sylgard 184 elastomer and curing agent (Dow Corning Corp), which was cast onto the silicon master and cured at $60^{\circ} \mathrm{C}$ overnight. The flow channels were cut to size and inlets and outlets were punched using a $1 \mathrm{~mm} \varnothing$ punch (Harris Uni-core, Sigma-Aldrich). After bonding to the chip, a flow channel of $6,000 \times 500 \times 50 \mu \mathrm{m}$ was prepared with a second channel entering from the side.

\subsubsection{PDMS bonding}

Chips were rinsed and sonicated extensively with acetone, ethanol and MilliQ water, and dried prior to UV-ozone exposure (UV/Ozone Procleaner plus, Bioforce Nanosciences) for at least $20 \mathrm{~min}$. After UV exposure, the chips were rinsed with ethanol and water, and dried under a stream of nitrogen. Both cutout PDMS flow channels and cleaned chips were treated with oxygen plasma for $30 \mathrm{~s}$ at $40 \mathrm{~W}$ (Plasma prep II, SPI supplies) after which they were bonded immediately. The chips were placed on a hot plate for $10 \mathrm{~min}$ at $70{ }^{\circ} \mathrm{C}$ to increase the binding strength. Tygon Microbore tubing S- $54-\mathrm{HL}$ (VWR, $0.25 \mathrm{~mm}$ inner $\varnothing$ and $0.75 \mathrm{~mm}$ outer $\varnothing$ ) of $80 \mathrm{~cm}$ for the inlets and $40 \mathrm{~cm}$ for the outlets was inserted into the PDMS. The assembled $\mu$ SLB electrophoresis chip was placed in an oven at $60^{\circ} \mathrm{C}$ for $1 \mathrm{~h}$. Leak-free operation was shown for flow rates up to $200 \mu \mathrm{L} / \mathrm{min}$.

\subsubsection{Lipid vesicle preparation}

MPPC (1-myristoyl-2-palmitoyl-sn-glycero-3-phosphocholine, Avanti) and DOPC (1,2-dioleoyl-sn-glycero-3-phosphocholine, Avanti) were stored as a 10 
$\mathrm{mg} / \mathrm{ml}$ stock solution in chloroform at $-20^{\circ} \mathrm{C}$. Biotin-cap-DOPE (1,2-dioleoyl-snglycero-3-phosphoethanolamine-N-(cap biotinyl), Avanti) was stored as 0.2 $\mathrm{mg} / \mathrm{ml}$ solution. Desired molar ratios were mixed in a glass vial, dried under a flow of nitrogen and kept under vacuum for at least $1 \mathrm{~h}$. The resulting film was resuspended by vortexing in MilliQ water at room temperature for DOPC and $50{ }^{\circ} \mathrm{C}$ for MPPC to form multilamellar vesicles (MLVs) at $1 \mathrm{mg} / \mathrm{ml}$. The MLV solution was extruded 11 times through a $100 \mathrm{~nm}$ polycarbonate membrane (Avanti) at room temperature for DOPC and $50^{\circ} \mathrm{C}$ for MPPC. The resulting large unilamellar vesicles (LUVs) were kept at room temperature and used within one week.

\subsubsection{SLB formation and functionalization}

Before SLB formation, the flow cells were washed with 2\% SDS (Sigma-Aldrich), then rinsed with MilliQ, and washed overnight with $2 \%$ Hellmanex $^{\mathrm{TM}}$ to activate the glass surface. Flow cells were mounted onto a heating plate using Scotch ${ }^{\text {TM }}$ tape and rinsed with MilliQ and PBS, while heating to $50^{\circ} \mathrm{C}$. Shortly before LUV incubation, the LUV solution was diluted to $0.5 \mathrm{mg} / \mathrm{ml}$ with PBS. The diluted LUV solution was passed through the flow cells through the secondary inlet for 30 min (10 $\mu \mathrm{L} / \mathrm{min}$; primary inlet $1 \mu \mathrm{L} / \mathrm{min}$ PBS) to allow adsorption and rupture of the vesicles on the chips. The chips were then washed with MilliQ $(100 \mu \mathrm{L} / \mathrm{min}$ secondary inlet, $10 \mu \mathrm{L} / \mathrm{min}$ primary inlet), after which freshly prepared $0.5 \mathrm{mM}$ hydroxymethylferrocene ( $\mathrm{FcMeOH}, \mathrm{Acros})$ was passed through the device (50 $\mu \mathrm{L} / \mathrm{min}$, both inlets). A potential difference of $2.0 \mathrm{~V}$ was applied over the device for $30 \mathrm{~min}$ to induce electrophoresis in the SLBs. Subsequently, the chips were cooled rapidly on a heat exchanger to fix the surface gradient. The flow cells were then rinsed with MilliQ. Bovine serum albumin (Sigma) $50 \mathrm{mg} / \mathrm{ml}(0.5 \mathrm{ml}$, $10 \mu \mathrm{L} / \mathrm{min}$ ) was passed through the flow cells to form an antifouling layer on the tubing. The flow cells were rinsed with PBS. SAv with Alexa Fluor 488 label (Thermo Fischer) $20 \mu \mathrm{g} / \mathrm{ml}$ was passed through the secondary inlet $(10 \mu \mathrm{L} / \mathrm{min}$, primary inlet $1 \mu \mathrm{L} / \mathrm{min})$. The flow cells were rinsed with PBS. The SAv-modified SLBs were inspected with fluorescence microscopy on an Olympus inverted IX71 epi-fluorescence research microscope with X-cite 120PC mercury vapor lamp as light source and a digital Olympus DR70 camera for image acquisition. For Alexa Fluor 488 , blue excitation $\left(460 \leq \lambda_{\mathrm{ex}} \leq 490 \mathrm{~nm}\right)$ and green emission $\left(505 \leq \lambda_{\mathrm{em}} \leq\right.$ $545 \mathrm{~nm}$ ) was filtered using the U-MWG2 Olympus filter cube. Glycans 2,3- and 2,6-S(LN) $)_{n}$-biotin $500 \mathrm{nM}$ solutions were passed through the primary inlet (10 
$\mu \mathrm{L} / \mathrm{min}$, secondary inlet $1 \mu \mathrm{L} / \mathrm{min}$ ), after which the flow cells were rinsed with PBS. During all steps, care was taken to ensure that no air bubbles entered the flow cell.

\subsubsection{Binding studies}

Solutions of IAV Puerto Rico/8/1934 mt. Sinai with R18 label were prepared in PBS with $200 \mu \mathrm{M}$ Zanamivir (GlaxoSmithKline). The virus solution was passed through the primary inlet of the flow cells $(1 \mu \mathrm{L} / \mathrm{min}$, secondary inlet $0.1 \mu \mathrm{L} / \mathrm{min})$ at a concentration of $10^{9} \mathrm{ml}^{-1}$ for $\mathrm{S}(\mathrm{LN})_{2}$ and $\mathrm{S}(\mathrm{LN})_{3}$, and $10^{10} \mathrm{ml}^{-1}$ for $\mathrm{S}(\mathrm{LN})_{1}$. The bound virus was then imaged with fluorescence microscopy. For R18 green excitation $\left(510 \leq \lambda_{\text {ex }} \leq 550 \mathrm{~nm}\right)$ and red emission $\left(\lambda_{\mathrm{em}}>590 \mathrm{~nm}\right)$ was filtered.

\subsubsection{Image analysis}

8-bit RGB fluorescence micrographs were aligned and cropped into corrals using a custom written MATLAB program. The fluorescence intensities of receptor and virus labels in matching pixels were listed. The receptor density in each pixel was calculated by $\rho_{R, i}=\bar{\rho}_{R} \cdot \frac{I_{i}-\bar{I}_{b g}}{\bar{I}-\bar{I}_{b g}}$, with $\bar{\rho}_{R}$ the average receptor density 2.77 $x$ the biotin\% in the SLB, based on a $60 \AA^{2}$ lipid footprint, ${ }^{48,49} I_{i}$ the local fluorescence intensity, $\bar{I}$ the average fluorescence intensity and $\bar{I}_{b g}$ the average fluorescence intensity of the background. The virus density was normalized between 0 and 1 by finding the maxima of $2 \mathrm{D}$ kernel estimations of the data points with the 1,000 lowest and $10 \%$ highest receptor densities.

Binding profiles were obtained by fitting Equation 4.1 with $K_{\mathrm{i}} / N_{\mathrm{A}} V_{\text {explore }}$ as fitting parameter, using the built-in fit function.

\subsubsection{Binding studies with QCM}

QCM-D measurements were performed using a QSense analyzer (Biolin Scientific). $\mathrm{SiO}_{2}$-coated sensors (QSX303, Biolin Scientific) were used. The sensors were cleaned using a $2 \%(\mathrm{w} / \mathrm{v})$ sodium dodecyl sulfate (SDS) solution and thoroughly rinsed with MilliQ water. Activation was performed with $20 \mathrm{~min}$ UV/ozone treatment, and the baseline was made flushing PBS buffer. Measurements were performed at $22{ }^{\circ} \mathrm{C}$ and operated with four parallel flow chambers, using two Ismatec peristaltic pumps with a fixed flow rate of 30 $\mu \mathrm{L} / \mathrm{min}$. Throughout this work, the fifth overtone $\left(\Delta f_{5}\right)$ was used for the normalized frequency and dissipation $\left(\Delta D_{5}\right)$. For SLB fabrication, vesicles of 
DOPC with $2 \%$ biotin-cap-DOPE were diluted to a concentration of $0.1 \mathrm{mg} / \mathrm{ml}$ in PBS directly before use. SLB formation was achieved by flowing this solution over a cleaned and activated $\mathrm{SiO}_{2}$ surface. The quality of the SLBs was monitored in situ by QCM-D, where a high quality SLB was characterized by $\Delta f_{5}$ $=-24 \pm 1 \mathrm{~Hz}$ and $\Delta D_{5}<0.5 \times 10^{-6}$. In a subsequent step, streptavidin (SAv) was bound to the biotin-modified SLB by flushing $0.5 \mu \mathrm{M}$ SAv in PBS solution, which was characterized by $\Delta f_{5}=-26 \pm 1$. In previous work, we found that biotin-capDOPE fractions in the SLB above $2 \%$ lead to a densely packed SAv layer on top of the $\mathrm{SLB}^{3}$. To immobilize the biotinylated $S(L N)_{n}$ receptors, a solution of each receptor with dummy receptor $(L N)_{2}$ in varying ratio was passed over the SLB after the stabilization of the frequency with PBS. The final concentration of the glycans was always $1 \mu \mathrm{M}$ leading to a $\Delta f_{5}=-2 \pm 1$, depending on the glycans used.

All the titration of PR8 were performed on a biotinylated-SLB functionalized with $\mathrm{S}(\mathrm{LN})_{\mathrm{n}}$. PR8 in a concentration range of 8.31 to $166 \mathrm{pM}$ with $100 \mu \mathrm{M}$ Zanamivir in PBS was flushed over the SLBs for 40 min. For each titration step, approximately $90 \%$ of the total frequency shift was obtained within the first 30 min. This suggests that the chosen time was sufficient for the viruses to approach thermodynamic equilibrium at these concentrations. Interestingly, at the highest concentration hardly any more virus bound to the surface, indicating that the surface became fully covered.

For a negative control of virus binding on the gel state SLBs that were used in the MAP chip, the QCM-D chamber was heated to $40^{\circ} \mathrm{C}$ at the start of the experiment. We used vesicles of MPPC with $0.5 \%$ biotin-cap-DOPE that were diluted to a concentration of $0.5 \mathrm{mg} / \mathrm{ml}$ in PBS directly before use. The chamber was cooled to $22^{\circ} \mathrm{C}$ before the SAv solution was added.

\subsubsection{Binding studies with BLI}

Discontinuous glycan gradients were obtained by mixing $S(L N)_{n}$ glycans with the non-sialylated $(L N)_{2}$ to functionalise SAv biosensors $(n=8)$ (Fortebio). Binding studies were performed using a series of $0.83-16.6 \mathrm{pM}$ virus. Plateau values of binding were normalized and plotted as a function of glycan density (range 0 to $3.8 \mathrm{pmol} / \mathrm{cm}^{2}$, based on the density of SAv on biosensors, ${ }^{19}$ assuming residual valency 1 for rapidly bound $S A v^{48}$ ). All virus binding experiments were performed in duplo. 


\subsubsection{Code availability}

The MATLAB scripts used for analysis of the fluorescence microcopy data of the MAP platform and for fitting the data to the multivalent model are available upon request.

\subsubsection{Microarray studies}

This write-up complies with MIRAGE Glycan Array Guideline v 1.0.

Materials

Virus isolates were produced as described above. Oseltamivir was purchased from Sigma Aldrich [Cat\# SML1606].

Arrayer and printing surfaces

Compounds were printed on amine reactive, NHS activated glass slides (NEXTERION ${ }^{\circledR}$ Slide $\mathrm{H}$ ) from Schott Inc using a Scienion sciFLEXARRAYER S3 noncontact microarray printer equipped with a Scienion PDC80 nozzle (Scienion Inc). Glycans were dissolved in printing buffer (sodium phosphate, $250 \mathrm{mM}, \mathrm{pH}$ 8.5 ) at a concentration of $100 \mu \mathrm{M}$. Each compound was printed in replicates of 6 with a spot volume of $\sim 400 \mathrm{pL}$, at $20^{\circ} \mathrm{C}$ and $50 \%$ humidity. Slides were blocked with $5 \mathrm{mM}$ ethanolamine in Tris buffer $\left(\mathrm{pH} \mathrm{9,50} \mathrm{mM)} \mathrm{for} 1 \mathrm{~h}\right.$ at $50^{\circ} \mathrm{C}$ and rinsed with DI water after printing.

\section{Glycan microarray}

Quality control was performed using the plant lectins Erythrina cristagalli agglutinin (ECA, specific for terminal Gal), Sambuca nigra agglutinin (SNA, specific for 2,6-linked Neu5Ac) and Maackia Amurensis Lectin I (MAL-I, specific for 2,3-linked Neu5Ac). ${ }^{50}$

\section{Sample}

Virus was diluted with PBS-T (PBS $+0.1 \%$ Tween, $25 \mu \mathrm{L}$ ) and applied to the array surface in the presence of oseltamivir ( $200 \mathrm{nM})$ in a humidified chamber for $1 \mathrm{~h}$. It was followed by a succesive rinsing with PBS-T (PBS $+0.1 \%$ Tween), PBS and deionized water $(2 \mathrm{x})$ and dried by centrifugation and scanned immediately.

Detector and data processing

The stained slides were scanned using an Innopsys Innoscan 710 microarray scanner at the appropriate excitation wavelength. To ensure that all signals 
were in the linear range of the scanner's detector and to avoid any saturation of the signals various gains and PMT values were employed. Images were analyzed with Mapix software (version 8.1.0 Innopsys) and processed with our home written Excel macro. The average fluorescence intensity and SD was measured for each compound after exclusion of the highest and lowest intensities from the spot replicates $(n=4)$.

\subsubsection{Molecular dynamics}

Generation of 3D molecular models

The 3D structures of the $S(L N)_{n}$ glycans were generated using the carbohydrate builder on GLYCAM-Web (www.glycam.org/cb). A linker-biotin moiety was added at the reducing terminus of each glycan using UCSF Chimera. ${ }^{51}$

Initial 3D models of complexes of each glycan bound to tetrameric SAv were generated by superimposing the biotin moiety onto each of the four biotin residues in a SAv-biotin co-crystal structure (PDB ID: 3RY2).

Molecular dynamics simulations

All simulations were performed using the Amber18 software suite. ${ }^{52}$ Using tleap, the SAv-glycan 3D structures were placed in a cubic box of TIP5P $\mathrm{P}^{53}$ water with a 10 water buffer with counterions added to neutralize total charge of the system. The FF14SB, ${ }^{54}$ GLYCAM06j, ${ }^{55}$ and GAFF ${ }^{56}$ force fields were employed for the protein, glycan and spacer-biotin components, respectively. A non-bonded cut-off of $10.0 \AA$ was employed for van der Waals interactions, with electrostatics treated using the particle mesh Ewald model within the PMEMD module. ${ }^{57}$ Initial energy minimization (10,000 steps of steepest decent followed by 10,000 steps of conjugate gradient) was performed with a Cartesian restraint $\left(5 \mathrm{kcal} \mathrm{mol}^{-1}\right)$ applied to all solute heavy atoms. The same restraints were applied during a solvent equilibration phase ( $0.4 \mathrm{~ns}$ under $\mathrm{nPT}$ conditions at 300 $\mathrm{K})$, followed by a solute equilibration phase ( $1 \mathrm{~ns}, \mathrm{nPT}, 300 \mathrm{~K}$ ) during which the Cartesian restraints were retained on protein $\mathrm{C} \alpha$ atoms only. The coordinates from the last step of equilibration were used to start five independent $100 \mathrm{~ns}$ production runs for each of the six systems, in which no restraints were employed. 


\section{Analysis of the MD simulations}

The Ambertools program cpptraj was used to extract 1,000 snapshots from each of the five independent SAv-glycan to give a total of 5,000 individual snapshots comprising 20,000 unique shapes for each glycan (four glycans bound per tetrameric SAv).

\section{Modeling HA binding to the SAv-glycan surface}

Models for monomeric binding between a single HA trimer and one SAv-glycan were generated by aligning an HA trimer onto the terminus of each of the SAvglycans. This alignment was achieved by superimposing the Gal-residue in the HA-glycan crystal structure (either from PDB ID:1RVX or 3UBE, which are cocrystalized with 2,3-SLN or 2,6-SLN oligosaccharides, respectively) onto the penultimate residue (Gal) in the SA-glycan. Models containing atomic overlaps arising during alignment of the HA-SAv moieties were eliminated from further consideration. The percent of shapes ( $n=5,000$ per system) without HA-SAv overlaps and the angle between the aligned HA and SAv centerlines were calculated using in-house code. This code is available on https://github.com/gitoliver/GlycoProteinBuilder.

Modeling $1 \mathrm{SAv}$ - $2 \mathrm{HA}$ binding mode

For each single HA trimer and one SA-glycan model that was generated, the distance between the HA headgroup center and the SAv center line was calculated. The ability for each glycan to form the 1SAv - 2 HA binding mode was assessed by calculating the distance between the HA headgroup center (average value of each Sia C2 atom) and the SAv tetramer center line, then doubling the value. Any values that were less than a HA headgroup diameter $(5.6 \mathrm{~nm})$ were discarded.

\section{Modeling 2 SAv - 1 HA binding mode}

The separate distance between the centers of two SAv tetramers bound in two binding sites of a single HA trimer was computed using the MD computed shapes. Each glycan-biotin molecule shape from the MD simulation $(n=4,000)$ was compared against all other shapes placed in a second HA binding site (7.998E+6 combinations). Any shape combination that oriented the SAvs such that they would overlap with each other (distance $<5.2 \mathrm{~nm}$ ) were discarded. 
Pulling simulations to generate bidentate model

The ability for each glycan to form a bidentate binding mode was checked using a previously reported protocol..$^{33}$ Glycans with shapes that are theoretically long enough to span two binding sites on a $\mathrm{HA}(>45 \AA)$ were superimposed onto the corresponding HA crystal structure (3UBE for 2,6-SLN, 1RVX for 2,3-SLN) via the Sia-Gal motif. The Sia-Gal motif of the neighboring glycan in the SAv tetramer is then brought into the second binding site of the HA by adjusting the intervening glycosidic angles. If the second motif can be brought within $\sim 5 \AA$, the system is subjected to a pulling MD simulation. NMR distance restraints based on the position of the Sia-Gal in the corresponding crystal structure are gradually introduced over the course of a 100 ns MD simulation to pull the Sia-Gal disaccharide into the binding site. This is followed by a $100 \mathrm{~ns}$ simulation during which no restraints are used. Bidentate binding is deemed possible for a glycan if it is able span two binding sites and then remain bound during the unrestrained MD. 


\subsection{Appendices}

a

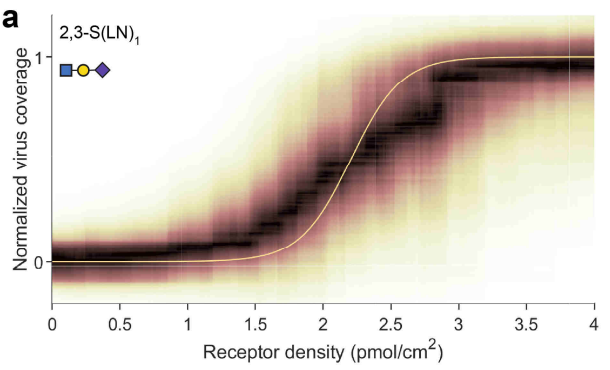

C

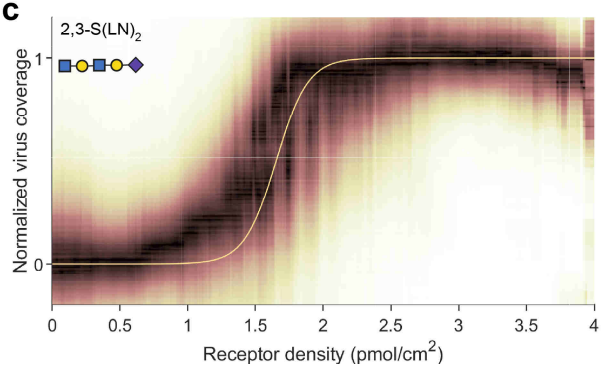

e

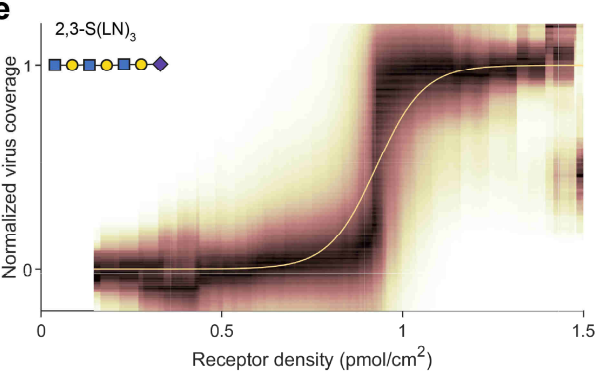

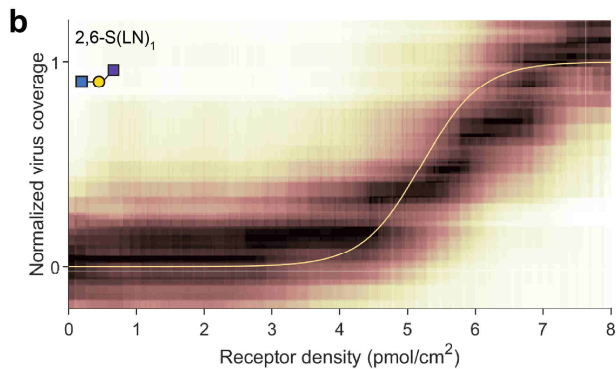

d

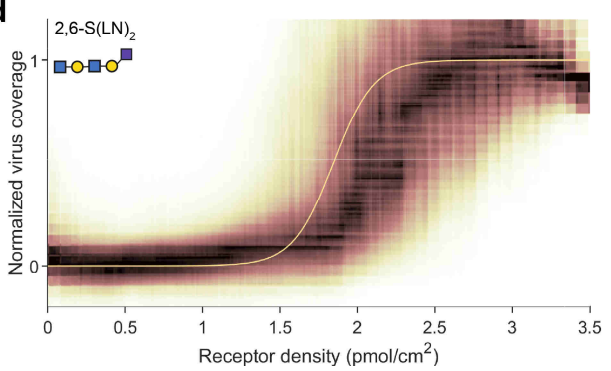

f

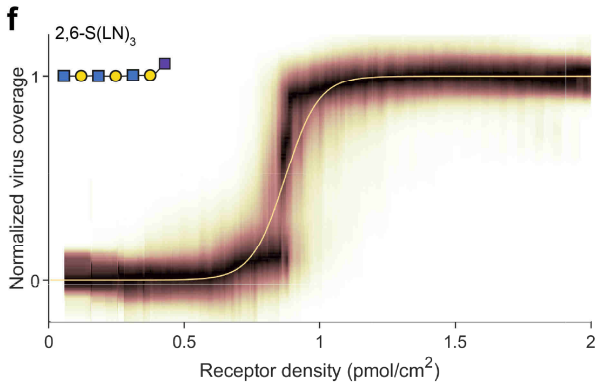

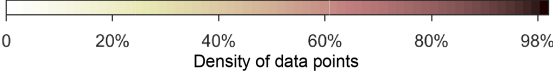

Figure 4.S1 | Binding profiles of influenza virus with multivalent affinity profiling. Data points are shown in a density map that was generated with a rolling average of $2001 \times 250$ points, a window of 40 , and a range of 0.1 in the $y$-direction unless otherwise specified. The density of data points is normalized for each window, because the density of data points is much higher on the left side of the graphs due to the exponential shape of the receptor gradients. The fitted curves are the same as in Figure 4.2d. a) 2,3-S(LN) $\mathrm{N}=1,530,544$ from 6 pairs of micrographs. b) 2,6-S(LN) $, N=92,480$ from 2 pairs of micrographs, range=0.3. c) 2,3-S(LN) $, N=406,912$ from 3 pairs of micrographs. d) 2,6$S(L N)_{2}, N=203,456$ from 3 pairs of micrographs. e) 2,3-S(LN) $)_{3} N=369,920$ from 3 pairs of micrographs. f) 2,6-S(LN) $3, \mathrm{~N}=406,912$ from 3 pairs of micrographs. 
a

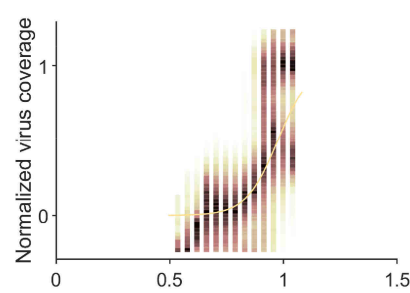

d Receptor density $\left(\mathrm{pmol} / \mathrm{cm}^{2}\right)$

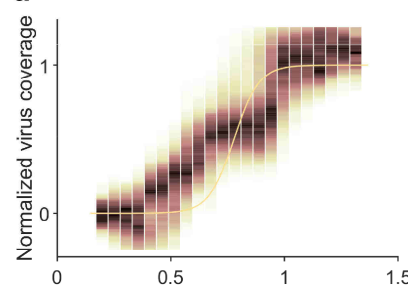

b

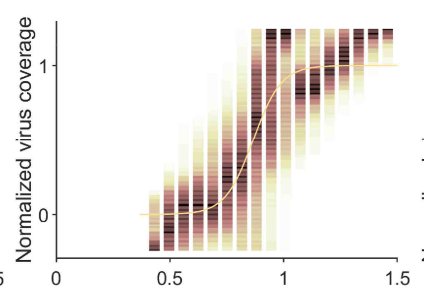

e Receptor density $\left(\mathrm{pmol} / \mathrm{cm}^{2}\right)$

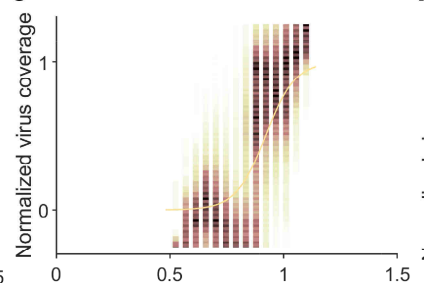

h Receptor density $\left(\mathrm{pmol} / \mathrm{cm}^{2}\right) \quad$ i

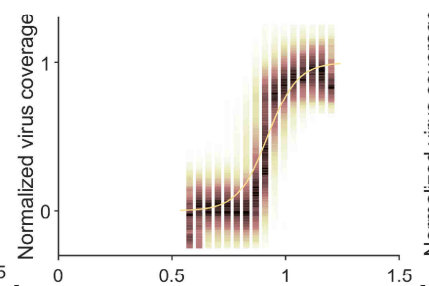

C

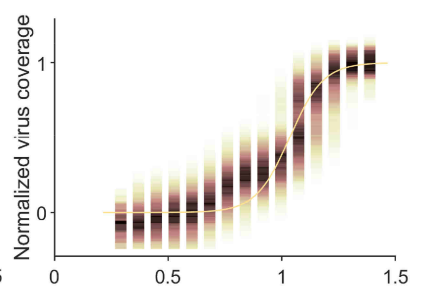

f Receptor density $\left(\mathrm{pmol} / \mathrm{cm}^{2}\right)$

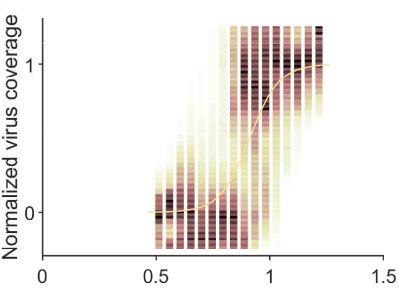

Receptor density $\left(\mathrm{pmol} / \mathrm{cm}^{2}\right)$
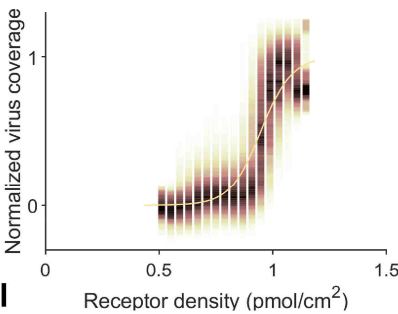

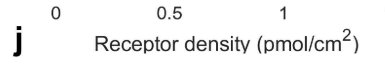

k
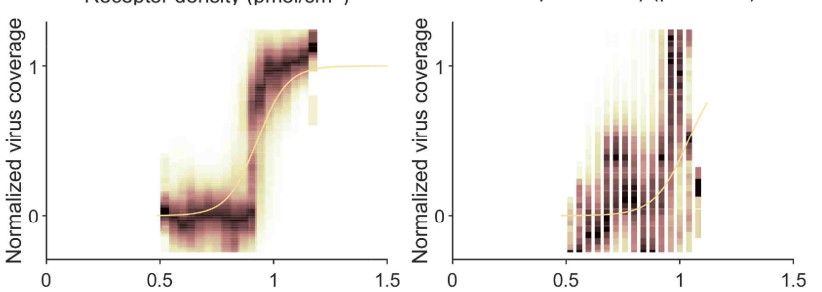

n

m Receptor density $\left(\mathrm{pmol} / \mathrm{cm}^{2}\right)$
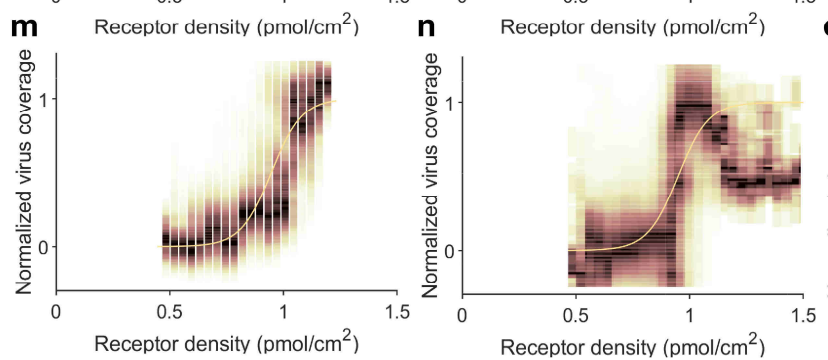

- Receptor density $\left(\mathrm{pmol} / \mathrm{cm}^{2}\right)$

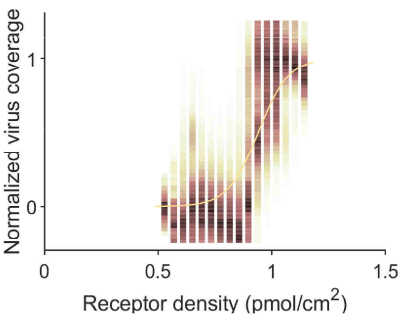



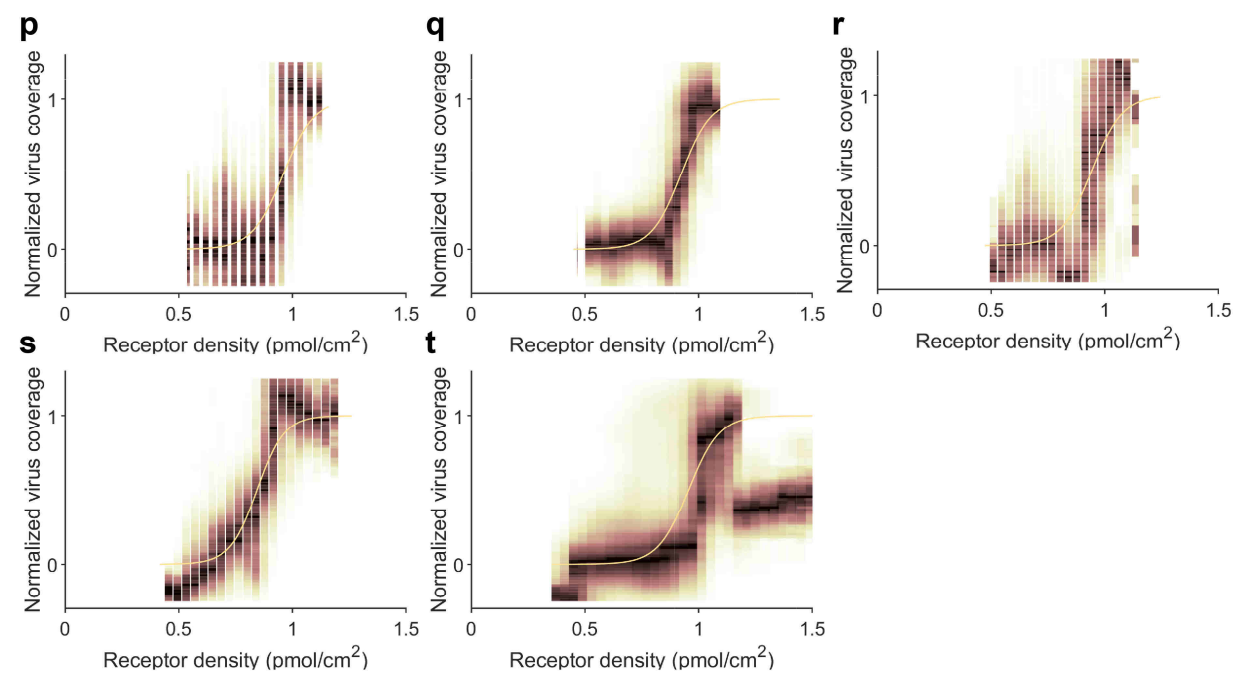

A Figure 4.S2 | Typical example of multiple binding profiles of PR8 obtained with multivalent affinity profiling. a-t) fitted binding profiles in 20 corrals that compose the binding profile of $2,3-\mathrm{S}(\mathrm{LN})_{3}$ shown in Figure $\mathrm{S} 1 \mathrm{e}$. 

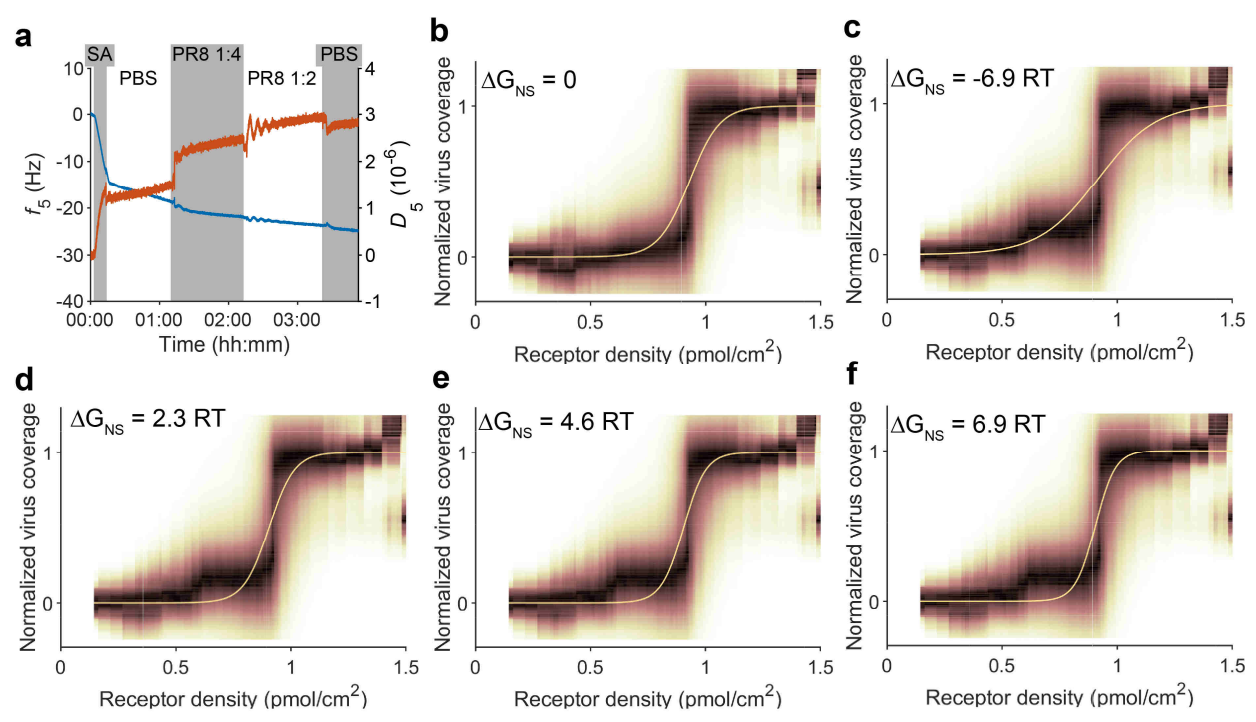

Figure 4.S3| Role of nonspecific binding in PR8 binding and data fitting. a) Frequency and dissipation shifts in QCM-D. As a negative control, an SLB of MPPC was functionalized with SA but not with glycans. PR8 was passed over the SLB at concentrations of $2.5 \cdot 10^{9} \mathrm{vp} / \mathrm{ml}$ and $5 \cdot 10^{9} \mathrm{vp} / \mathrm{ml}$, with $100 \mu \mathrm{M}$ Zanamivir. The $5^{\text {th }}$ overtone is shown. The virus binding to the SA-modified SLB is negligible. b) Binding profile of $\mathrm{PR} 8$ on 2,3-S(LN) $)_{3}$ fitted with Equation $\mathrm{S} 6$ using $\Delta \mathrm{G}_{\mathrm{NS}}=0$, so that it simplifies to Equation 1. Profile and fit are the same as in Figure S1e. c) The same binding profile fitted with $\Delta G_{N S}=-6.9 R T$. d) The same binding profile fitted with $\Delta G_{N S}=2.3 R T$. e) The same binding profile fitted with $\Delta G_{N S}=4.6 \mathrm{RT}$. f) The same binding profile fitted with $\Delta \mathrm{G}_{\mathrm{NS}}=6.9 \mathrm{RT}$.

\subsubsection{Derivation and fitting of theoretical binding model}

We derive an expression for the equilibrium binding constant ("avidity constant") of a ligand-coated virus particle binding to a receptor-coated surface. The expression is converted into a form that can be readily used by inputting experimental parameters, and applied to generate a Langmuir adsorption isotherm for virus binding (as a function of solution concentration and substrate receptor density).

\section{Avidity constant}

Consider viruses with a diameter of $d$. When the virus is adjacent to the receptor surface, then a fraction of the virus's outer shell is in contact with the 
surface. The contact area $A_{\text {contact }}$ is defined as the receptor surface area over which RBD-glycan pairs can be formed to a single virus particle. Furthermore, let $\sigma_{\mathrm{L}}$ be the number of ligands per unit area on the virus exterior, and $\sigma_{\mathrm{R}}$ be the number of receptors per unit area on the receptor surface. The receptors are assumed to be Poisson distributed and/or mobile on the receptor surface. The HA ligands on the virus surface are assumed to be uniformly distributed, and they are embedded in a rather densely packed corona. This assumption is based on the trimeric structure of HA with RBDs on adjacent trimers at approximately the same spacing as between RBDs on the same trimer. As a result, a given RBD has limited configurational flexibility, and may only bind to the nearest glycan on the adjacent receptor surface.

Reference 27 outlines the statistical thermodynamics of multivalent binding in the regime where ligands and receptors are short and with limited configurational flexibility, so that each may only bind to the nearest partners within a given microscopic range. Drawing from their derivations as a starting point, we define the binding partition function for our present scenario of virus binding as follows:

$Q_{b i n d}=\left(1+e^{-\Delta G_{l i g} / R T}\right)^{\widetilde{N}} e^{-\Delta G_{N S} / R T} e^{\mu^{\circ} / R T}$

where

- $\tilde{\mathrm{N}}=A_{\text {contact }} \cdot \min \left(\sigma_{\mathrm{R}}, \sigma_{\mathrm{L}}\right)$ is the average total number of possible simultaneous bonds that may be formed between a bound virus particle and the receptor surface;

- $\Delta G_{\text {lig }}$ is the free energy for making a single ligand-receptor (here: RBDglycan) bond;

- $R$ is the ideal gas constant;

- $T$ is the temperature;

- $\Delta G_{N S}$ is any overall non-specific virus-surface interaction free energy

- $\mu^{\circ}$ is the chemical potential of the viruses in solution at standard 1 molar concentration. 
Inclusion of the chemical potential weight in $Q_{\text {bind }}$ accounts for the thermodynamic cost of drawing in a virus particle from the bulk solution in order to bind it to the receptor surface.

We now translate Equation 4.51 into the avidity constant $K_{\mathrm{av}}$. First, the total ligand-receptor binding free energy $\Delta G_{\text {lig }}$ contains several contributions:

$\Delta G_{\text {lig }}=-R T \ln \left(\frac{K_{i}^{\circ}}{N_{A} h_{\text {bind }} A_{\text {reach }}}\right)+\Delta G_{\text {lig, cnf }}$

The first term contains the contribution from $K_{\mathrm{i}}^{\circ}$, the ligand-receptor binding equilibrium constant in free solution. The quantity $V_{\text {explore }}$ is the local volume of space that the RBD and glycan binding units may explore, given that they are tethered to the virus and sensor substrate, respectively. Thus, the quantity $1 / N_{\mathrm{A}} V_{\text {explore }}$ is the "effective molarity" of glycan on the surface, from the perspective of a single RBD on a bound virus. The second term $\Delta G_{\text {lig,nf }}$ accounts for any additional free energy cost for making a ligand-receptor bond, e.g. if the ligand or receptor experience any further restriction on their internal degrees of freedom, or if there are additional ligand-receptor steric interactions.

Next, the chemical potential $\mu$ is related to the molar concentration [ $V$ ] of virus in solution by

$\frac{\mu}{R T}=\ln \left(N_{A} V_{e x}[V]\right)+$ excess chemical potential terms,

where $V_{\mathrm{ex}}$ is the occupied volume of a virus particle usually taken to be $d^{\beta}$ or $\frac{4 \pi}{3}(d / 2)^{3}$. The standard chemical potential in Equation 4.S1 is simply Equation $4 . \mathrm{S} 3$ evaluated at the standard concentration of $[\mathrm{V}]=1 \mathrm{M}$ (one molar), assuming ideal solution conditions:

$\frac{\mu^{\circ}}{R T}=\ln \left(N_{A} V_{e x}[1 M]\right)$

Lastly, we assume that the viruses pack on the surface at a maximum possible packing fraction of $\phi$ (a value between zero and one). The amount of surface area excluded by a virus when it is bound to the surface is $A_{\mathrm{ex}}$.

The equilibrium avidity constant $K_{\mathrm{av}}$ is obtained from $Q_{\text {bind }}$ by the thermodynamic relations 
$\Delta G_{\text {bind }}^{\circ}=-R T \ln Q_{\text {bind }}=-R T \ln \left([1 M] K_{a v}\right)$

With this, Equation 4.S1, and the previous substitutions, we arrive at:

$K_{a v}=N_{A} V_{e x} e^{-\Delta G_{N S} / R T}\left(1+\frac{K_{i}}{N_{A} V_{\text {explore }}} e^{-\Delta G_{\text {lig,cnf }} / R T}\right)^{\widetilde{N}}$

This expression yields $K_{\mathrm{av}}$ in units of inverse molarity $\left(\mathrm{dm}^{3} / \mathrm{mol}\right)$ by expressing length-derived quantities in $\mathrm{dm}$, and $K_{\mathrm{i}}$ in $\mathrm{M}^{-1}$. The free energy quantities can be expressed in any energy units compatible with those chosen for $R T$.

Virus adsorption isotherm

We now derive the adsorption profile (isotherm) for virus binding on a receptorcoated substrate surface, given that the substrate has a density $\sigma_{R}$ of receptors, and the viruses are at a bulk solution (or flow) concentration of $\left[\mathrm{V}^{\circ}\right.$.

The avidity constant is related to the equilibrium concentrations of free and bound multivalent viruses in a solution via:

$K_{a v}=\frac{[R]_{\text {occupied }}}{[V]_{\text {free }}[R]_{\text {free }}}$.

Here, $[R]_{\text {occupied }}$ is the concentration of receptors "occupied" on the substrate: either bound to or sterically blocked by a substrate-bound virus particle. The quantity [ $V]_{\text {free }}$ is the concentration of viruses that are not bound (i.e. free in solution), and $[R]_{\text {free }}$ is the concentration of substrate receptors that are available for binding-e.g. neither bound to a ligand, nor sterically blocked by a nearby bound virus.

The first step we take is to assume that the number of viruses in solution far exceeds the number that can possibly bind to the substrate. This condition can be met if, for example, the volume of our "virus solution" is very large compared to the size of the substrate that the viruses can bind to, or if the substrate is being exposed to a continuous flow of a virus solution at a fixed concentration. In these cases, the solution concentration of unbound viruses is assumed to be a constant with respect to how many bind to the substrate. Therefore, the previous equation simplifies to

$K_{a v}=\frac{[R]_{\text {occupied }}}{[V]^{\circ}[R]_{\text {free }}}$, 
where $[\mathrm{V}]^{\circ}$ is the input bulk solution concentration of viruses.

When a virus binds to the substrate, it blocks all receptors that are directly below it over an area of $A_{\text {ex }}$. Thus, the concentration of occupied receptors $[R]_{\text {occupied }}$ in the system is just the number $N_{\text {bound }}$ of bound viruses times the average number of receptors they each occupy, $A_{\mathrm{ex}} \sigma_{\mathrm{R}}$, divided by the volume $V_{\text {sys }}$ of the sample:

$[R]_{\text {occupied }}=\frac{N_{\text {bound }} A_{e x} \sigma_{R}}{V_{\text {sys }} \phi}$,

where $\sigma_{\mathrm{R}}$ is the number of receptors per unit area on the substrate. Dividing by $\phi$ here incorporates the packing efficiency of the binding viruses. Similarly, the concentration $[R]_{\text {free }}$ of free receptors is the concentration of all receptors $[R]$ $=A_{\mathrm{sfc}} \sigma_{\mathrm{R}} / V_{\text {sys }}$ (where $A_{\mathrm{sfc}}$ is the total area of the substrate) minus the concentration $[R]_{\text {occupied }}$ that is occupied:

$[R]_{\text {free }}=\frac{1}{V_{\text {sys }}}\left(A_{s f c} \sigma_{R}-\frac{N_{\text {bound }} A_{e x} \sigma_{R}}{\phi}\right)$,

Inserting these definitions into Equation 4.58 yields

$K_{a v}=\frac{1}{[V]^{\circ}}\left[\frac{\frac{1}{\phi}\left(\frac{N_{\text {bound }} A_{e x}}{A_{s f c}}\right)}{1-\frac{1}{\phi}\left(\frac{N_{\text {bound }} A_{e x}}{A_{s f c}}\right)}\right]$.

Defining $\theta=N_{\text {bound }} A_{\text {ex }} / A_{\text {sfc }}$ as the fraction of binding positions occupied by viruses at equilibrium, we arrive at

$K_{a v}=\frac{1}{[V]^{\circ}}\left[\frac{(\theta / \phi)}{1-(\theta / \phi)}\right]$.

Solving for $\theta$ yields

$\theta=\phi\left(\frac{K_{a v}[V]^{\circ}}{1+K_{a v}[V]^{\circ}}\right)$.

This may be equivalently expressed in number of bound viruses per unit surface area by

$\rho_{\text {bound }}=\frac{\phi}{A_{e x}}\left(\frac{K_{a v}[V]^{\circ}}{1+K_{a v}[V]^{\circ}}\right)$. 
Application of the theoretical model to experimental data

To determine the number of interactions at the threshold, we applied the theoretical model to the MAP data. Normalized virus coverage is plotted against receptor density for each corral (Figure 4.S2). The binding profiles varied strongly in shape, partly due to stochastic variations and partly due to defects in the SLBs. Binding profiles on multiple corrals, from multiple positions in the microchannel and multiple experiments are combined into a single binding profile on which the fitting is performed (Figure 4.S1). This afforded a better fit than the average of each corral. The virus concentration [ $V$ ] and diameter were characterized with nanoparticle tracking analysis (NTA) and $V_{\text {ex }}$ was calculated from the virus diameter. We used $[V]=10^{9}$ particles $/ \mathrm{ml}$ and $d=112 \mathrm{~nm}$. We found that the threshold receptor density did not change over time (data not shown), so that the profile of normalized virus binding as function of $\sigma_{R}$ was the same as the profile of $\theta$ and $\phi=1$ could be used.

To test the role of nonspecific binding on the SLBs in the MAP chip, we prepared an SLB of MPPC with $0.5 \%$ biotin-cap-DOPE in a $\mathrm{SiO}_{2}$-coated sensor in QCM-D and modified it with SA but not with glycans (Figure 4.S3a). We passed PR8 over the $S L B$ in concentrations of $2.5 \cdot 10^{9} \mathrm{vp} / \mathrm{ml}$ and $5 \cdot 10^{9} \mathrm{vp} / \mathrm{ml}$ in the presence of $100 \mu \mathrm{M}$ Zanamivir. Apart from some baseline drift and noise at solution changes, the shifts in frequency upon passing PR8 over the SLB are negligible and may be attributed to viscosity or refractive index changes as they are rapidly reversed upon changing the solution to PBS. We tested the effect of $\Delta G_{\mathrm{NS}}$ on the fits by fitting the binding profile of $2,3-S(L N)_{3}$ with different values of $\Delta G_{N S}$ (Figure 4.S3b-f) and found that positive (repulsive) values for $\Delta G_{N S}$ lead to steeper binding profiles, whereas negative values lead to less steep profiles. A two-parameter fit with $\Delta G_{\mathrm{NS}}$ as extra free parameter appeared too sensitive towards noise around the threshold receptor density which led to it fitting strongly negative values for $\Delta G_{N S}$ to the steep profiles of $S(L N)_{3}$. Upon visual inspection of the binding profiles and fits in Figure 4.S1, $S(L N)_{3}$ appears to have a positive $\Delta G_{N S}$, whereas $S(L N)_{1}$ may have a negative $\Delta G_{N s}$. Possibly, the longer glycans provide a stronger steric repulsion. This means that no uniform assumption can be made with regards to $\Delta G_{N S}$ as it may likely vary with contact area and glycan density as well. 
Because we did not observe nonspecific binding, we assume in this study for the sake of simplicity that $\Delta G_{\mathrm{NS}}=0$. Because $\Delta G_{\mathrm{lig}, \mathrm{cnf}}$, and $K_{\mathrm{i}}^{\circ}$ could not be fitted independently, we use $K_{i, e f f}=K_{i}^{\circ} e^{-\Delta G_{\text {lig,cnf }} / R T}$ to account for any differences between the $K_{\mathrm{i}}^{\circ}$ of a free glycan and RBD in solution and the effective $K_{\mathrm{i}}$ at the surface, such as steric repulsion between SAv on the surface and HA. This way, Equation 4.S13 simplifies to

$\theta=\frac{K_{a v}[V]^{\circ}}{1+K_{a v}[V]^{\circ}}$

and Equation 4.S6 simplifies to Equation 4.1.

We used the maximum contact area to fit the profile of $\theta$ as function of $\sigma_{\mathrm{R}}$ with $K_{\text {i,eff }} / N_{\mathrm{A}} V_{\text {explore }}$ as free parameter. The model in Figure $4.5 \mathrm{c}$ implies that the maximum contact area is bound by the maximum distance at which a glycan can reach an RBD. We used molecular dynamics to determine the minimum and maximum distance between sialic acid in the RBD and biotin in the binding pocket of SAv (Figure 4.S4a and S4b). The maximum contact area that can be reached by glycans of this length is shown in Figure 4.S4c.

Due to the curvature of the virus, a larger contact area not only means that RBDs are farther from the surface, but also that the angle between HA and SAv is larger (Figure 4.S4d). We hypothesized that glycan-RBD interactions farther away from the surface would be less favorable. Instead we found with molecular dynamics that the most favorable angle between HA and SAv is between $30^{\circ}$ and $100^{\circ}$ and interactions are much less likely when HA and SAv are in a straight line (Figure 4.5b). At virus contact areas of $2.4-4.0 \%$, the angle of $\mathrm{HA}$ is $17.8-23.1^{\circ}$. We estimate that $\mathrm{SAv}$ can tilt approximately $16^{\circ}$, allowing a maximum angle between $\mathrm{HA}$ and $\mathrm{SAv}$ of $34-39^{\circ}$. This suggests that for all glycans in this study, the RBDs close to the edge of the contact area contribute most strongly to the average $K_{\mathrm{i}, \text { eff. }}$. We estimate that $V_{\text {explore }}$ is the volume between a half-sphere with the minimum glycan length as radius and one with the maximum as radius (Figure 4.S4e). Using the fitted values for $K_{\mathrm{i} \text {,eff }} / N_{\mathrm{A}} V_{\text {explore, we }}$ calculate the average $K_{\mathrm{i}, \text { eff }}$ for each glycan type (Figure 4.S4f). 
a

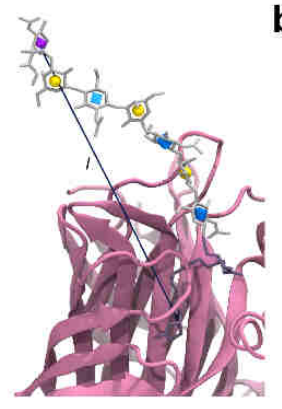

d

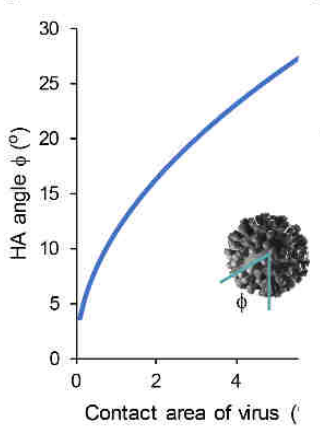

b

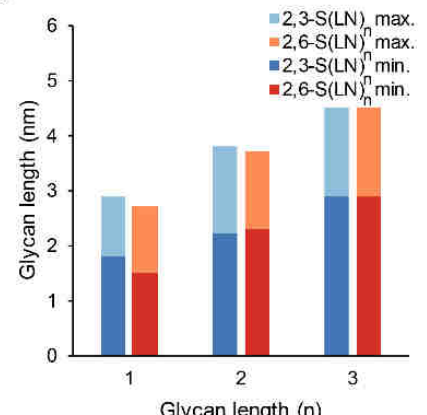

e

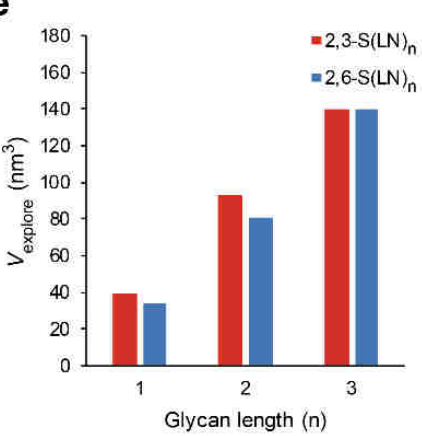

C

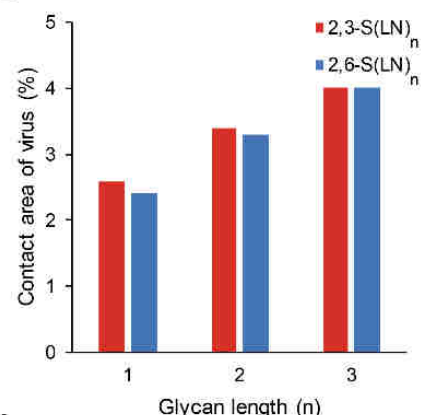

f

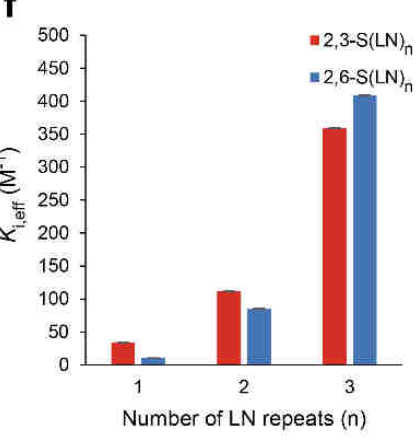

Figure 4.S4| Estimation of the virus contact area of PR8 and explored volume of receptors from glycan length to determine the effective monovalent equilibrium constants of the HA-glycan interactions. a) Molecular dynamics snapshot showing how the distance between biotin and sialic acid was determined. b) Minimum and maximum distances between biotin and sialic acid for different glycan types as determined with MD. c) Contact area of PR8 corresponding to the maximum length of the different glycans. Longer glycans can reach RBDs that are farther away from the surface. d) The maximum angle of $\mathrm{HA}$ with respect to the surface as function of contact area. $0^{\circ}$ is perpendicular to the surface. If SAv can tilt $16^{\circ}$ with respect to the surface, the maximum angle between $\mathrm{HA}$ and $\mathrm{SAv}$ is $34-39^{\circ}$, which is less than the optimal angle (Figure 4.5b), suggesting that the RBDs close to the edge of the contact area contribute most strongly to the average $K_{\mathrm{i}, \text { eff. It }}$ is therefore reasonable to assume the maximum value for $A_{\text {contact. }}$ e) Values for $V_{\text {explore }}$ based on the volume between two half-spheres with the minimum and maximum glycan length as radius. f) Values for $K_{\mathrm{i} \text {,eff calculated }}$ from $V_{\text {explore }}$ and the fitted values for $K_{\mathrm{i} \text {,eff }} /\left(N_{\mathrm{A}} V_{\text {explore }}\right)$. 


\subsubsection{Modes of HA-glycan complexation}

Molecular modelling of HA-glycan binding

MD simulations were employed to explore the possible shapes that the $S(L N)_{n}$ glycan-linker-biotin molecule can adopt when it is bound to the SAv tetramer, thus to understand the influence of glycan length, structure and presentation on the virus binding. The percentage of shapes for each SAv-immobilized glycan type that could allow HA binding were determined (Figure 4.5b). In general, as the glycan becomes shorter, it displays fewer shapes compatible with HA binding, particularly in the case of 2,6-S(LN) , which correlates with the observed avidity data (Figure 4.2e). The decrease in shapes compatible with HA binding corresponds to the decrease in conformational entropy of the glycans, but ignores interactions between the HA head and LN repeats. ${ }^{17}$

To take into account the orientational constraints of the HA and SAv molecules that arise from their immobilization on the virus and array surfaces, respectively, the angle between the center lines of the HA and SAv were determined (illustrated in Figure 4.5a). Based on a simple geometric model (Figure 4.5c) for a spherical virus interacting with glycans projecting from a flat SAv surface, the allowable angular range for the glycan relative to the surface depends on the glycan length and the virion radius. The MD data were used to provide estimates of the average glycan length for each type of glycan bound to $S A v$, and the radius of the virion was measured with nanoparticle tracking analysis. With these values, the allowable angles ranged from 0 to $17.8^{\circ}$ for the shortest glycans and up to $23.1^{\circ}$ for the longest. Additionally, we estimate that SAv may tilt up to $16^{\circ}$, so that HA-SAv angles of up to $39^{\circ}$ are allowable. Even with the tilting of SAv, these ranges represent only a small subset of the orientations that would be allowable were the glycan not immobilized on a flat surface.

For each glycan, the total number of orientations with allowable angles correlates well with the observed dependence on receptor densities (Figure 4.2); the shorter glycans, especially $2,6-\mathrm{S}(\mathrm{LN})_{1}$, have fewer acceptable glycan poses, and therefore require a higher receptor density to bind the virus. The longer glycans having a higher number of acceptable orientations can bind the virus at lower glycan densities. Examining the dependence of the acceptable angle range as a function of sialoside linkage type, the theoretical data also 
indicate that the 2,6-S(LN) ${ }_{1}$ glycan has markedly fewer acceptable orientations compared to the $2,3-\mathrm{S}(\mathrm{LN})_{1}$ glycan, consistent with the density dependence in Figure 4.2.

Multivalent binding mode of $1 \mathrm{HA}$ with multiple SAv molecules The geometry corresponding to a single HA trimer binding to two separate SAvglycan complexes ( $1 \mathrm{HA}-2 \mathrm{SAv}$ ) was generated from the MD simulations of each glycan type. The separation distance between the centers of two SAv tetramers bound in two separate binding sites of the HA trimer was computed (Figure 4.S5a). Each shape from the MD simulation $(n=4000)$ was compared against all other shapes in the second HA binding site (7.998.106 combinations). Any shape combination that oriented the SAvs such that they would overlap (distance $<5.2 \mathrm{~nm}$ ) were discarded. The cumulative fraction of shapes that were not discarded are reported in Figure 4.S5b as function of the distance between the approaching SAvs. 
a

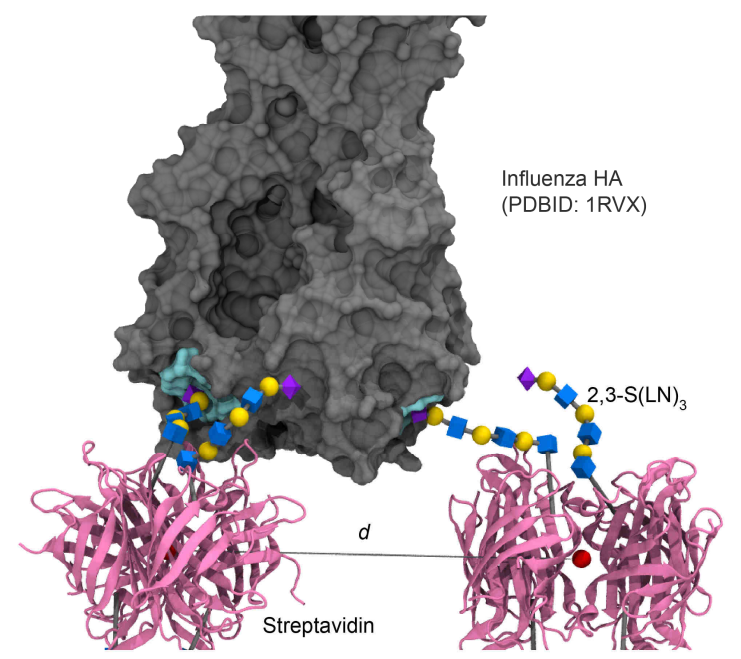

b

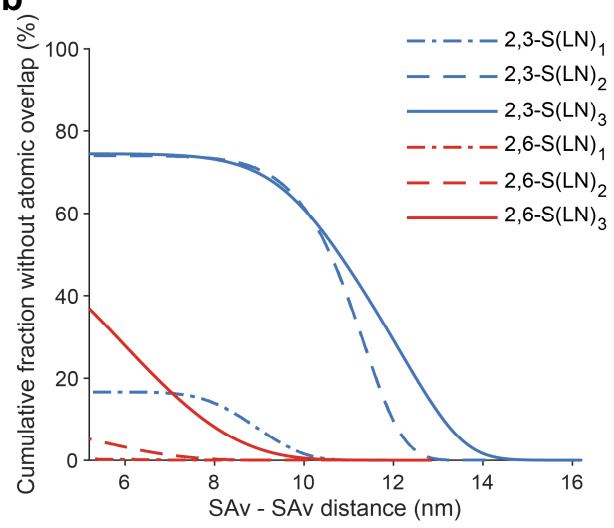

C

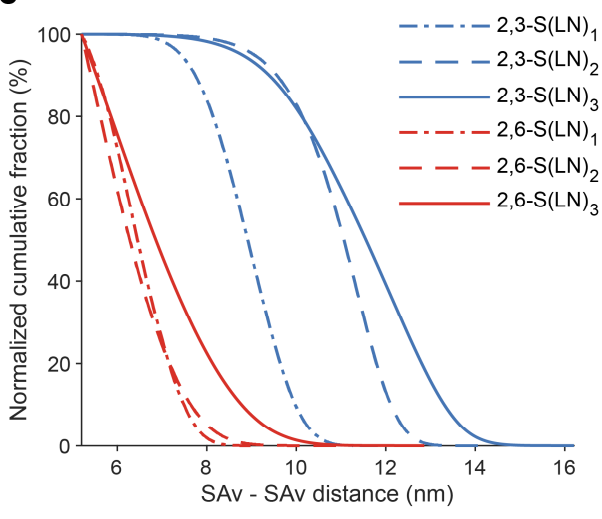

Figure 4.S5| The availability of the 1 HA - 2 SAv mode depends on the receptor type and the distance between the SAvs. a) Illustration of the $1 \mathrm{HA}-2 \mathrm{SAv}$ multivalent binding mode. The biotinylated glycan from two SAv proteins (pink ribbon) are aligned into two independent binding sites of an influenza HA. The availability of this binding mode is directly related to density of SAv on the surface and the shapes adopted by each glycan. b) Cumulative fraction of glycan shapes for which glycans on different SAv proteins can bind to the same HA trimer without atomic overlap as function of the distance between the two SAvs. The fraction is shown as percentage of all possible shapes. c) The cumulative fraction of glycan shapes from Figure 4.S5b is normalized to show the differences in how the availability of this mode depends on the SAv-SAv distance for each glycan type. 
a

b

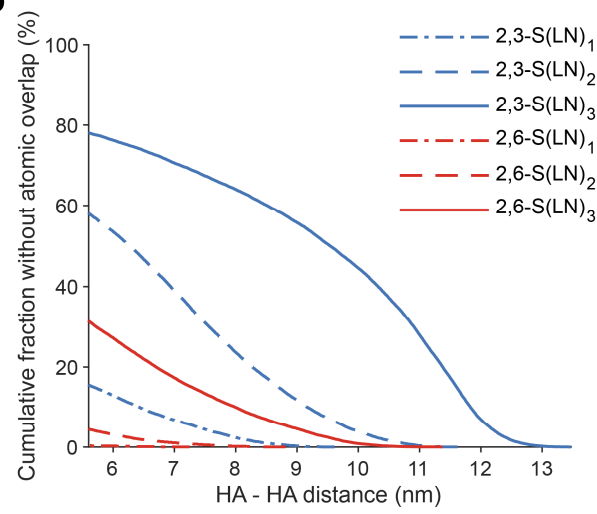

C

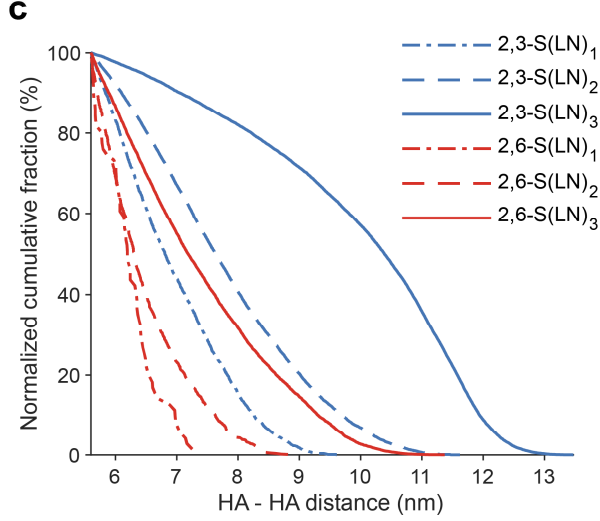

Figure 4.S6| The availability of the 2 HA - 1 SAv mode depends on the receptor type and the distance between the SAvs. a) Illustration of the $2 \mathrm{HA}-1 \mathrm{SAv}$ multivalent binding mode. The biotinylated glycans from one SAv protein (pink) are aligned into two binding sites on different HA trimers. The availability of this binding mode is directly related to density of HA on the virus and the shapes adopted by each glycan. b) Cumulative fraction of glycan shapes for which glycans on one SAv proteins can bind to different $\mathrm{HA}$ trimers without atomic overlap as function of the distance between two the HAs. For 2,6-S(LN) $)_{1}$ this mode is hardly available even between close-packed HAs. c) The cumulative fraction of glycan shapes from Figure $4.56 \mathrm{~b}$ is normalized to show the differences in how the availability of this mode depends on the HA-HA distance for each glycan type. 
Multivalent binding mode of $2 \mathrm{HA}$ trimers with glycans on a single SAv The ability for each glycan to form the $1 \mathrm{SAv}-2 \mathrm{HA}$ binding mode (Figure 4.S6a) was assessed by calculating the distance between the HA headgroup center (average value of each Sia C2 atom) and the SAv tetramer center line, then doubling the value. Any values that were less than the diameter of a HA headgroup $(5.6 \mathrm{~nm})$ were discarded. The cumulative fraction of shapes that were not discarded are reported in Figure $4.56 \mathrm{~b}$ as function of the distance between the approaching HAs.

Bidendate binding mode of 1HA - 1SAV

The linear inter-binding site distance on an HA trimer is $\sim 45 \AA$, however, this ignores that any bidendate molecule will have to span over/around the head group and that the binding sites require a particular orientation of the Sia-Gal. Following a previously reported protocol ${ }^{33}$, glycans with shapes that are theoretically long enough to span two binding sites on a HA ( $>45 \AA$ ) were checked by superimposing one of the binding motifs into the crystal structure, and then adjusting the intervening glycosidic linkages within known ranges to bring the second binding motif into the second binding site, without causing atomic overlaps between the glycan or the SAv and the HA headgroup. Once the second motif is within $\sim 5 \AA$, restraints are gradually introduced over the course of an MD simulation to pull the Sia-Gal disaccharide in into the binding position observed in the corresponding crystal structure. Bidendate binding is deemed possible for a glycan if it is able span two binding sites and then remain bound during an extended (> $100 \mathrm{~ns}$ ), unrestrained MD.

The analysis found that only the SAv bearing 2,6-S(LN) $)_{3}$ glycans was capable of forming a bidendate interaction with a single HA (Figure 4.S7). The other glycans $\left(2,6-\mathrm{S}(\mathrm{LN})_{1}, 2,6-\mathrm{S}(\mathrm{LN})_{2}\right.$ and all of the 2,3-linked glycans) were either too short or, in the case of $2,3-\mathrm{S}(\mathrm{LN})_{3}$, the required orientation of both motifs could not be achieved. 


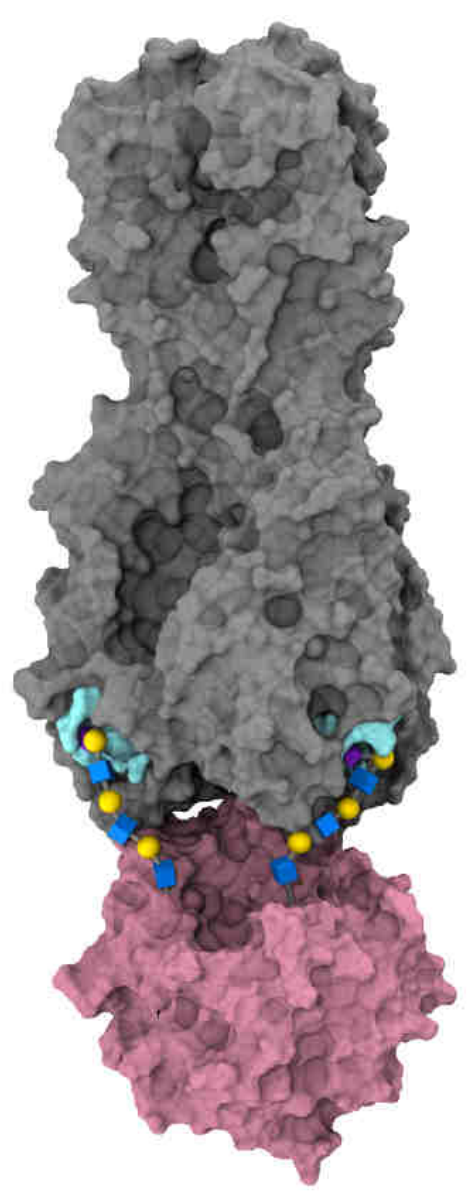

Figure 4.S7| 3D model of bidendate binding between 2,6-S(LN) $3-S A v$ and HA (PDB ID: 3UBE). The model was generated by superimposing to the Sia-Gal motif in the crystal structure and using a previously reported protocol to bring the second glycan into the second binding site of HA. The bidendate mode was stable throughout an unrestrained 100 ns MD simulation. 


\subsection{References}

1. Schaapveld, M., Aleman, B. M. P., van Eggermond, A. M., Janus, C. P. M., Krol, A. D. G., van der Maazen, R. W. M., Roesink, J., Raemaekers, J. M. M., de Boer, J. P., Zijlstra, J. M., van Imhoff, G. W., Petersen, E. J., Poortmans, P. M. P., Beijert, M., Lybeert, M. L., Mulder, I., Visser, O., Louwman, M. W. J., Krul, I. M., Lugtenburg, P. J. \& van Leeuwen, F. E. Emergence of a Novel Swine-Origin Influenza A (H1N1) Virus in Humans. N. Engl. J. Med. 360, 2605-2615 (2009).

2. Wu, F., Zhao, S., Yu, B., Chen, Y.-M., Wang, W., Song, Z.-G., Hu, Y., Tao, Z.-W., Tian, J.-H., Pei, Y.-Y., Yuan, M.-L., Zhang, Y.-L., Dai, F.-H., Liu, Y., Wang, Q.-M., Zheng, J.-J., Xu, L., Holmes, E. C. \& Zhang, Y.-Z. A new coronavirus associated with human respiratory disease in China. Nature 579, 265-269 (2020).

3. Herfst, S. \& Fouchier, R. Epidemiological and genetic investigations of human-to-human transmission of zoonotic influenza viruses. Eurosurveillance 19, 20840 (2014).

4. Maines, T. R., Chen, L. M., Van Hoeven, N., Tumpey, T. M., Blixt, O., Belser, J. A., Gustin, K. M., Pearce, M. B., Pappas, C., Stevens, J., Cox, N. J., Paulson, J. C., Raman, R., Sasisekharan, R., Katz, J. M. \& Donis, R. O. Effect of receptor binding domain mutations on receptor binding and transmissibility of avian influenza H5N1 viruses. Virology 413, 139-147 (2011).

5. Di lorio, D., Verheijden, M. L., van der Vries, E., Jonkheijm, P. \& Huskens, J. Weak Multivalent Binding of Influenza Hemagglutinin Nanoparticles at a SialoglycanFunctionalized Supported Lipid Bilayer. ACS Nano 13, 3413-3423 (2019).

6. de Graaf, M. \& Fouchier, R. A. M. Role of receptor binding specificity in influenza A virus transmission and pathogenesis. EMBO J. 33, 823-841 (2014).

7. Paulson, J. C. \& de Vries, R. P. H5N1 receptor specificity as a factor in pandemic risk. Virus Res. 178, 99-113 (2013).

8. Matrosovich, M., Tuzikov, A., Bovin, N., Gambaryan, A., Klimov, A., Castrucci, M. R., Donatelli, I. \& Kawaoka, Y. Early Alterations of the Receptor-Binding Properties of H1, $\mathrm{H} 2$, and H3 Avian Influenza Virus Hemagglutinins after Their Introduction into Mammals. J. Virol. 74, 8502-8512 (2000).

9. Koel, B. F., Burke, D. F., Bestebroer, T. M., Vliet, S. van der, Zondag, G. C. M., Vervaet, G., Skepner, E., Lewis, N. S., Spronken, M. I. J., Russell, C. A., Eropkin, M. Y., Hurt, A. C., Barr, I. G., Jong, J. C. de, Rimmelzwaan, G. F., Osterhaus, A. D. M. E., Fouchier, R. A. M. \& Smith, D. J. Substitutions Near the Receptor Binding Site Determine Major Antigenic Change During Influenza Virus Evolution. Science 342, 976-979 (2013).

10. Yamada, S., Suzuki, Y., Suzuki, T., Le, M. Q., Nidom, C. A., Sakai-Tagawa, Y., Muramoto, Y., Ito, M., Kiso, M., Horimoto, T., Shinya, K., Sawada, T., Kiso, M., Usui, T., Murata, T., Lin, Y., Hay, A., Haire, L. F., Stevens, D. J., Russell, R. J., Gamblin, S. J., Skehel, J. J. \& Kawaoka, Y. Haemagglutinin mutations responsible for the binding of H5N1 influenza A viruses to human-type receptors. Nature 444, 378-382 (2006).

11. Richard, M., van den Brand, J. M. A., Bestebroer, T. M., Lexmond, P., de Meulder, D., Fouchier, R. A. M., Lowen, A. C. \& Herfst, S. Influenza A viruses are transmitted via the air from the nasal respiratory epithelium of ferrets. Nat. Commun. 11, 766 (2020). 


\section{Chapter 4}

12. Memoli, M. J., Bristol, T., Proudfoot, K. E., Sally Davis, A., Dunham, E. J. \& Taubenberger, J. K. In vivo evaluation of pathogenicity and transmissibility of influenza A(H1N1)pdm09 hemagglutinin receptor binding domain 222 intrahost variants isolated from a single immunocompromised patient. Virology 428, 21-29 (2012).

13. Russell, C. J., Hu, M. \& Okda, F. A. Influenza Hemagglutinin Protein Stability, Activation, and Pandemic Risk. Trends Microbiol. 26, 841-853 (2018).

14. Vahey, M. D. \& Fletcher, D. A. Influenza A virus surface proteins are organized to help penetrate host mucus. elife 8, 1-24 (2019).

15. Chandrasekaran, A., Srinivasan, A., Raman, R., Viswanathan, K., Raguram, S., Tumpey, T. M., Sasisekharan, V. \& Sasisekharan, R. Glycan topology determines human adaptation of avian H5N1 virus hemagglutinin. Nat. Biotechnol. 26, 107-113 (2008).

16. Zhang, W., Shi, Y., Lu, X., Shu, Y., Qi, J. \& Gao, G. F. An Airborne Transmissible Avian Influenza H5 Hemagglutinin Seen at the Atomic Level. Science 340, 1463-1467 (2013).

17. Ji, Y., White, Y. J., Hadden, J. A., Grant, O. C. \& Woods, R. J. New insights into influenza A specificity: an evolution of paradigms. Curr. Opin. Struct. Biol. 44, 219-231 (2017).

18. Nemanichvili, N., Tomris, I., Turner, H. L., McBride, R., Grant, O. C., van der Woude, R., Aldosari, M. H., Pieters, R. J., Woods, R. J., Paulson, J. C., Boons, G. J., Ward, A. B., Verheije, M. H. \& de Vries, R. P. Fluorescent Trimeric Hemagglutinins Reveal Multivalent Receptor Binding Properties. J. Mol. Biol. 431, 842-856 (2019).

19. Xiong, X., Coombs, P. J., Martin, S. R., Liu, J., Xiao, H., McCauley, J. W., Locher, K., Walker, P. A., Collins, P. J., Kawaoka, Y., Skehel, J. J. \& Gamblin, S. J. Receptor binding by a ferrettransmissible H5 avian influenza virus. Nature 497, 392-396 (2013).

20. Vachieri, S. G., Xiong, X., Collins, P. J., Walker, P. a, Martin, S. R., Haire, L. F., Zhang, Y., McCauley, J. W., Gamblin, S. J. \& Skehel, J. J. Receptor binding by H10 influenza viruses. Nature 511, 475-477 (2014).

21. Müller, M., Lauster, D., Wildenauer, H. H. K., Herrmann, A. \& Block, S. Mobility-Based Quantification of Multivalent Virus-Receptor Interactions: New Insights into Influenza A Virus Binding Mode. Nano Lett. 19, 1875-1882 (2019).

22. Benton, D. J., Martin, S. R., Wharton, S. A. \& McCauley, J. W. Biophysical measurement of the balance of influenza A hemagglutinin and neuraminidase activities. J. Biol. Chem. 290, 6516-6521 (2015).

23. Martinez-Veracoechea, F. J. \& Frenkel, D. Designing super selectivity in multivalent nanoparticle binding. Proc. Natl. Acad. Sci. U.S.A. 108, 10963-10968 (2011).

24. Dubacheva, G. V., Curk, T., Auzély-Velty, R., Frenkel, D. \& Richter, R. P. Designing multivalent probes for tunable superselective targeting. Proc. Natl. Acad. Sci. U.S.A. 112, 5579-5584 (2015).

25. Van Weerd, J., Krabbenborg, S. O., Eijkel, J., Karperien, M., Huskens, J. \& Jonkheijm, P. On-Chip Electrophoresis in Supported Lipid Bilayer Membranes Achieved Using Low Potentials. J. Am. Chem. Soc. 136, 100-103 (2014).

26. Krabbenborg, S. O., van Weerd, J., Karperien, M., Jonkheijm, P. \& Huskens, J. Locked-in Biomimetic Surface Gradients that are Tunable in Size, Density and Functionalization. ChemPhysChem 15, 3460-3465 (2014). 
27. Curk, T., Bren, U. \& Dobnikar, J. Bonding interactions between ligand-decorated colloidal particles. Mol. Phys. 116, 3392-3400 (2018).

28. Schuck, P. \& Zhao, H. in Life Sci. (eds. Mol, N. J. \& Fischer, M. J. E.) 627, 15-54 (Humana Press, 2010).

29. Martinez-Veracoechea, F. J. \& Leunissen, M. E. The entropic impact of tethering, multivalency and dynamic recruitment in systems with specific binding groups. Soft Matter 9, 3213 (2013).

30. Varilly, P., Angioletti-Uberti, S., Mognetti, B. M. \& Frenkel, D. A general theory of DNAmediated and other valence-limited colloidal interactions. J. Chem. Phys. 137, 094108 (2012).

31. Vahey, M. D. \& Fletcher, D. A. Low-Fidelity Assembly of Influenza A Virus Promotes Escape from Host Cells. Cell 176, 281-294 (2019).

32. Harris, A., Cardone, G., Winkler, D. C., Heymann, J. B., Brecher, M., White, J. M. \& Steven, A. C. Influenza virus pleiomorphy characterized by cryoelectron tomography. Proc. Natl. Acad. Sci. U.S.A. 103, 19123-19127 (2006).

33. Peng, W., de Vries, R. P., Grant, O. C., Thompson, A. J., McBride, R., Tsogtbaatar, B., Lee, P. S., Razi, N., Wilson, I. A., Woods, R. J. \& Paulson, J. C. Recent H3N2 Viruses Have Evolved Specificity for Extended, Branched Human-type Receptors, Conferring Potential for Increased Avidity. Cell Host Microbe 21, 23-34 (2017).

34. Benton, D. J., Wharton, S. A., Martin, S. R. \& McCauley, J. W. Role of Neuraminidase in Influenza A(H7N9) Virus Receptor Binding. J. Virol. 91, 1-10 (2017).

35. Stevens, J., Blixt, O., Tumpey, T. M., Taubenberger, J. K., Paulson, J. C. \& Wilson, I. A. Structure and Receptor Specificity of the Hemagglutinin from an H5N1 Influenza Virus. Science 312, 404-410 (2006).

36. Long, J. S., Mistry, B., Haslam, S. M. \& Barclay, W. S. Host and viral determinants of influenza A virus species specificity. Nat. Rev. Microbiol. 17, 67-81 (2019).

37. Van Riel, D., Munster, V. J., de Wit, E., Rimmelzwaan, G. F., Fouchier, R. A. M., Osterhaus, A. D. M. E. \& Kuiken, T. H5N1 Virus Attachment to Lower Respiratory Tract. Science 312, 399-399 (2006).

38. Walther, T., Karamanska, R., Chan, R. W. Y., Chan, M. C. W., Jia, N., Air, G., Hopton, C., Wong, M. P., Dell, A., Malik Peiris, J. S., Haslam, S. M. \& Nicholls, J. M. Glycomic Analysis of Human Respiratory Tract Tissues and Correlation with Influenza Virus Infection. PLoS Pathog. 9, e1003223 (2013).

39. Seaman, G., Knox, R., Nordt, F. \& Regan, D. Red cell agins. I. Surface charge density and sialic acid content of density-fractionated human erythrocytes. Blood 50, 1001-1011 (1977).

40. Mammen, M., Dahmann, G. \& Whitesides, G. M. Effective Inhibitors of Hemagglutination by Influenza Virus Synthesized from Polymers Having Active Ester Groups. Insight into Mechanism of Inhibition. J. Med. Chem. 38, 4179-4190 (1995).

41. Kesimer, M., Ehre, C., Burns, K. A., Davis, C. W., Sheehan, J. K. \& Pickles, R. J. Molecular organization of the mucins and glycocalyx underlying mucus transport over mucosal surfaces of the airways. Mucosal Immunol. 6, 379-392 (2013). 


\section{Chapter 4}

42. McAuley, J. L., Corcilius, L., Tan, H.-X., Payne, R. J., McGuckin, M. A. \& Brown, L. E. The cell surface mucin MUC1 limits the severity of influenza A virus infection. Mucosal Immunol. 10, 1581-1593 (2017).

43. Huang, M. L., Cohen, M., Fisher, C. J., Schooley, R. T., Gagneux, P. \& Godula, K. Determination of receptor specificities for whole influenza viruses using multivalent glycan arrays. Chem. Commun. 51, 5326-5329 (2015).

44. Jansen, A. J. G., Spaan, T., Low, H. Z., Di Iorio, D., van den Brand, J., Tieke, M., Barendrecht, A., Rohn, K., van Amerongen, G., Stittelaar, K., Baumgärtner, W., Osterhaus, A., Kuiken, T., Boons, G.-J., Huskens, J., Boes, M., Maas, C. \& van der Vries, E. Influenza-induced thrombocytopenia is dependent on the subtype and sialoglycan receptor and increases with virus pathogenicity. Blood Adv. 4, 2967-2978 (2020).

45. Li, T., Liu, L., Wei, N., Yang, J. Y., Chapla, D. G., Moremen, K. W. \& Boons, G. J. An automated platform for the enzyme-mediated assembly of complex oligosaccharides. Nat. Chem. 11, 229-236 (2019).

46. Lau, K., Thon, V., Yu, H., Ding, L., Chen, Y., Muthana, M. M., Wong, D., Huang, R. \& Chen, $X$. Highly efficient chemoenzymatic synthesis of $\beta 1-4$-linked galactosides with promiscuous bacterial $\beta 1$-4-galactosyltransferases. Chem. Commun. 46, 6066 (2010).

47. Rimmelzwaan, G. F., Baars, M., Claas, E. C. J. \& Osterhaus, A. D. M. E. Comparison of RNA hybridization, hemagglutination assay, titration of infectious virus and immunofluorescence as methods for monitoring influenza virus replication in vitro. $J$. Virol. Methods 74, 57-66 (1998).

48. Dubacheva, G. V., Araya-Callis, C., Geert Volbeda, A., Fairhead, M., Codée, J., Howarth, M. \& Richter, R. P. Controlling Multivalent Binding through Surface Chemistry: Model Study on Streptavidin. J. Am. Chem. Soc. 139, 4157-4167 (2017).

49. Nagle, J. F. \& Tristram-Nagle, S. Structure of lipid bilayers. Biochim. Biophys. Acta-Rev. Biomembr. 1469, 159-195 (2000).

50. Haseley, S. R., Talaga, P., Kamerling, J. P. \& Vliegenthart, J. F. G. Characterization of the carbohydrate binding specificity and kinetic parameters of lectins by using surface plasmon resonance. Anal. Biochem. 274, 203-210 (1999).

51. Pettersen, E. F., Goddard, T. D., Huang, C. C., Couch, G. S., Greenblatt, D. M., Meng, E. C. \& Ferrin, T. E. UCSF Chimera-A visualization system for exploratory research and analysis. J. Comput. Chem. 25, 1605-1612 (2004).

52. Case, D. A., Ben-Shalom, I. Y., Brozell, S. R., Cerutti, D. S., Cheatham, T. E., Cruzeiro, V. W. D., Darden, T. A., Duke, R. E., Ghoreishi, D., Gilson, M. K., Gohlke, H., Goetz, A. W., Greene, D., Harris, R., Homeyer, N., Izadi, S., Kovalenko, A., Kurtzman, T., Lee, T. S., LeGrand, S., Li, P., Lin, C., Liu, J., Luchko, T., Luo, R., Mermelstein, D. J., Merz, K. M., Miao, Y., Monard, G., Nguyen, C., Nguyen, H., Omelyan, I., Onufriev, A., Pan, F., Qi, R., Roe, D. R., Roitberg, A., Sagui, C., Schott-Verdugo, S., Shen, J., Simmerling, C. L., Smith, J., Salomon-Ferrer, R., Swails, J., Walker, R. C., Wang, J., Wei, H., Wolf, R. M., Wu, X., Xiao, L., York, D. M. \& Kollman, P. A. AMBER 2018, University of California, San Francisco. (2018).

53. Mahoney, M. W. \& Jorgensen, W. L. A five-site model for liquid water and the reproduction of the density anomaly by rigid, nonpolarizable potential functions. $J$. 
Chem. Phys. 112, 8910-8922 (2000).

54. Maier, J. A., Martinez, C., Kasavajhala, K., Wickstrom, L., Hauser, K. E. \& Simmerling, C. ff14SB: Improving the Accuracy of Protein Side Chain and Backbone Parameters from ff99SB. J. Chem. Theory Comput. 11, 3696-3713 (2015).

55. Kirschner, K. N., Yongye, A. B., Tschampel, S. M., González-Outeiriño, J., Daniels, C. R., Foley, B. L. \& Woods, R. J. GLYCAM06: A generalizable biomolecular force field. Carbohydrates. J. Comput. Chem. 29, 622-655 (2008).

56. Wang, J., Wolf, R. M., Caldwell, J. W., Kollman, P. A. \& Case, D. A. Development and testing of a general amber force field. J. Comput. Chem. 25, 1157-1174 (2004).

57. Salomon-Ferrer, R., Götz, A. W., Poole, D., Le Grand, S. \& Walker, R. C. Routine Microsecond Molecular Dynamics Simulations with AMBER on GPUs. 2. Explicit Solvent Particle Mesh Ewald. J. Chem. Theory Comput. 9, 3878-3888 (2013). 


\section{Chapter 5}

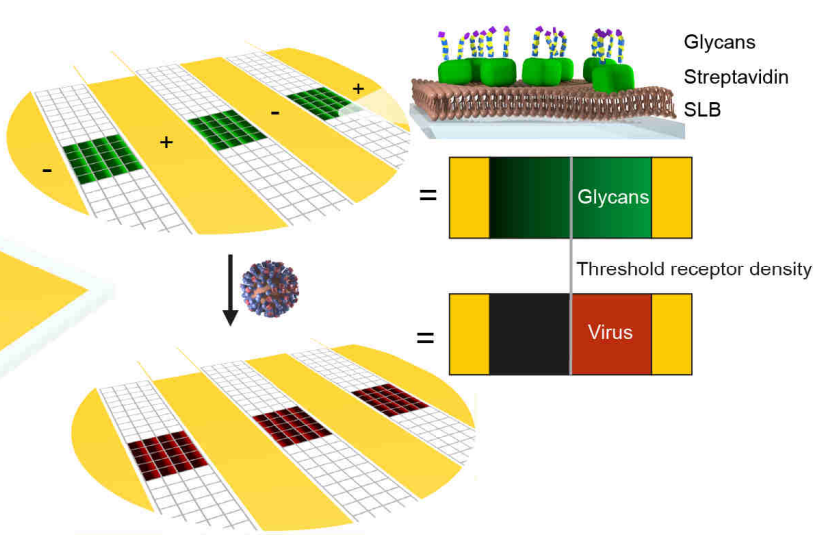

\section{Highlights}

- Receptor density gradients are used to visualize the superselective binding of IAV

- Binding profiles as a function of receptor density are used to quantitatively assess multivalent binding

- Gradient design is proposed that avoids overestimation of the receptor density

- The threshold receptor density increases with the flow rate 


\section{Direct visualization of the superselective binding of influenza viruses}

The influenza A virus (IAV) interacts with the glycocalyx of host cells through its surface proteins hemagglutinin (HA) and neuraminidase (NA). Quantitative biophysical measurements of these interactions may help to understand these interactions at the molecular level with the long-term aim to predict influenza infectivity and answer other biological questions. A method was developed to measure virus binding profiles on receptor density gradients to determine the threshold receptor density. This chapter shows that imaging of IAVs on receptor density gradients allows the direct visualization of their superselective binding. We show how the multivalent binding of IAVs can be quantitatively assessed using this method if the receptor density gradients are prepared around the threshold receptor density without crowding at the higher densities. The threshold receptor density increases strongly with increasing flow rate, showing that the superselective binding of IAV is influenced by shear force. This method of visualization and quantitative assessment of superselective binding does not only allow comparative studies of IAV-receptor interactions, but also more fundamental studies of how superselectivity arises and is influenced by experimental conditions.

Part of this chapter has been published in:

Overeem, N.J., Hamming, P.H., Tieke, M., Van der Vries, E., Huskens, J., - submitted 


\subsection{Introduction}

Influenza A viruses (IAVs) originate in wild waterfowl and have repeatedly caused outbreaks in livestock and deadly zoonotic infections. ${ }^{1}$ Adaptation to human hosts has led to four pandemics since 1918 followed by regular seasonal epidemics. ${ }^{2}$ The initiation of an infection is governed by complex interactions between the surface proteins of the virus and the glycocalyx of the host (Chapter 2). Biophysical measurements of IAV can help to understand these interactions by quantifying physical parameters that contribute to the virushost cell interactions. ${ }^{3-5}$ Effective use of these measurements requires an understanding of how the measured parameters relate to the interactions of IAV with the glycocalyx on the molecular level. ${ }^{6}$

The surface proteins of IAV are hemagglutinin (HA) and neuraminidase (NA). HA can bind sialic acid-terminated glycans with millimolar affinities. ${ }^{7} \mathrm{NA}$ is an enzyme that binds the same receptors with comparable affinities and cleaves the bond between sialic acid and the penultimate sugar monomer, which is usually galactose..$^{3,8,9}$ The opposing actions of HA and NA have a functional balance that ensures sufficient avidity while maintaining the dynamicity that the virus needs to proliferate. ${ }^{10-12}$ Avian influenza viruses bind preferentially to glycans where the sialic acid is linked to the penultimate galactose by an $\alpha 2,3-$ linkage (2,3-SLN), whereas human influenza viruses favor the $\alpha 2,6$-linkage (2,6SLN). ${ }^{13,14}$ Structural factors such as the length and branching of glycans also affect the specificity of the virus (Chapter 4 ).

The receptor density determines whether a virus can bind or not, as multiple glycan-HA interactions are needed for stable attachment. ${ }^{7,15}$ If a particle needs multiple interactions for stable attachment, its binding depends nonlinearly on the number of receptors that are available in the contact area. ${ }^{16}$ The resulting bias towards high receptor densities is called "superselectivity", which is characterized by a sigmoidal increase in binding around a threshold receptor density. ${ }^{17-19}$

Biolayer interferometry (BLI) is an optical technique that is used to address both the receptor specificity of IAV and the receptor density dependence of the virussurface interaction. ${ }^{7,20} \mathrm{BLI}$ typically uses streptavidin (SAv)-modified sensor tips that are functionalized with biotinylated glycans, glycopolymers or 
glycoproteins. ${ }^{7,12}$ The density of receptors is controlled by varying the loading time $^{12}$ or concentration, ${ }^{7}$ or by diluting the receptors with a dummy receptor (Chapter 4). The virus binding is measured in solutions with a single, fixed concentration of virus to obtain binding profiles as a function of relative receptor loading. The resulting BLI curves are used as a relative measure of IAV avidity. The insight that this technique can offer is limited by the limited control over the surface chemistry of the sensor tips and the constant virus concentration.

Glycan microarrays provide an efficient technique to evaluate the receptor specificity of IAV. ${ }^{21,22}$ Small islands of several to hundreds of different glycan types are printed on a surface in an array so that the binding to each glycan type can be measured relative to the others. ${ }^{23,24}$ The binding of IAV or recombinant $\mathrm{HA}$ is quantified using fluorescent labels. ${ }^{25,26}$ While this technique is more efficient in evaluating the receptor specificity than BLI, it offers even less control over the surface chemistry and does not address receptor density dependence.

Surface gradients of receptors allow evaluating virus binding as a function of receptor density in a single image. Spatial gradients of physicochemical properties are widely used to improve the efficiency of analysis, design and discovery. ${ }^{27-33}$ Our group developed methods using interdigitated electrodes on glass chips to form electrochemical surface density gradients in self-assembled monolayers ${ }^{34,35}$ and electrophoretic gradients in supported lipid bilayers (SLBs). ${ }^{36}$ By using lipids that are in the gel state at room temperature, gradients have been formed at elevated temperatures and locked-in by cooling. ${ }^{37}$ The resulting gradients were then post-functionalized and used as a receptor density gradient. . $^{31,38}$

In Chapter 4, we introduced a method, called "multivalent affinity profiling" (MAP), to measure the avidity of influenza viruses that uses receptor density gradients to obtain binding profiles as a function of receptor density. The binding profiles were acquired using fluorescence microscopy. Although this technique is generally not suitable for quantification, here the use of internal standards and normalization allow MAP to determine threshold receptor densities that can be compared with those obtained using other platforms. When the maximum receptor density on the sensor tips of BLI is known, this technique can be used to determine the threshold receptor density similar to 
MAP, though less efficiently. ${ }^{39}$ The binding profiles obtained with MAP were used to determine the threshold receptor density, and we applied a model to determine the number of interactions and their individual contributions. Broader application of this method in dynamic measurements requires further understanding of how superselective binding is visualized and how the threshold receptor density depends on experimental conditions.

This chapter shows that receptor density gradients can be used to make superselective binding of IAV visible in a single image. These images can be analyzed to quantitatively assess multivalent IAV binding and study the effect of shear force on the binding. First, we show that the broad and continuous range of receptor densities on a gradient allows visualization of superselective binding. Image analysis of fluorescence micrographs is a powerful method to construct binding profiles. The threshold receptor density is shown to be a characteristic quantity for a superselective particle-surface interaction. After that, the influence of flow cell design and experimental conditions on the accuracy of the measured binding profiles is studied. A model is described to account for the influence of flow rate on the threshold receptor density.

\subsection{Results and discussion}

\subsubsection{Visualization and quantification of virus binding on receptor gradients}

Receptor gradients for studying the binding of IAV were prepared in a microfluidic chip as shown in Figure 5.1a. The sensing area in the MAP chip is divided into three positions with a total of 21-48 corrals, depending on the chip layout and the alignment of the microchannel. Using a previously described method, ${ }^{38}$ we formed SLBs from vesicles of zwitterionic MPPC (1-myristoyl-2palmitoyl-sn-glycero-3-phosphocholine) with $0.3-0.8 \%$ biotin-DOPE (1,2dioleoyl-sn-glycero-3-phosphoethanolamine- $\mathrm{N}$-(cap biotinyl)), which has a negative charge. Electrophoretic gradients were formed by applying a potential over the SLBs. The resulting biotin gradients were subsequently modified with fluorescently labelled streptavidin (SAv) and biotinylated glycans $\left(2,6-\mathrm{S}(\mathrm{LN})_{3}\right.$, Figure 5.1b). The fluorescent SAv signals the presence of $2,6-S(L N)_{3}$, so that the co-localization of fluorescently labelled IAV and 2,6-S(LN) $)_{3}$ can be imaged to obtain binding profiles and determine the threshold receptor density (Figure 5.1a). 
a

b

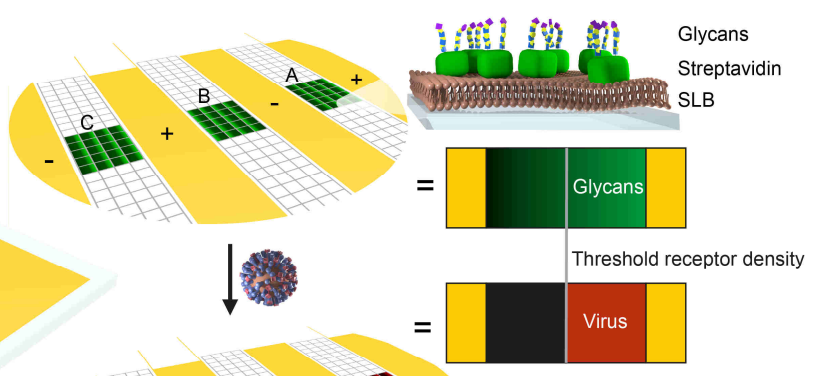

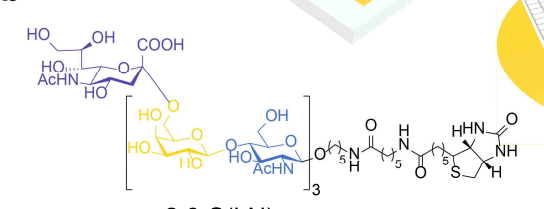

$2,6-\mathrm{S}(\mathrm{LN})_{3}$

C

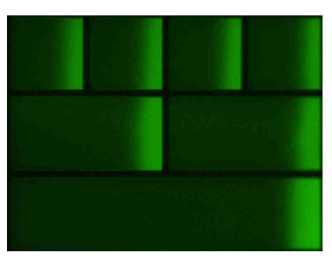

e

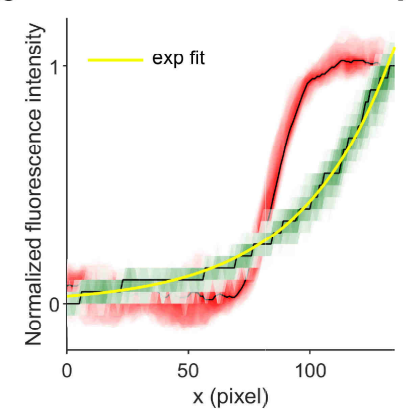

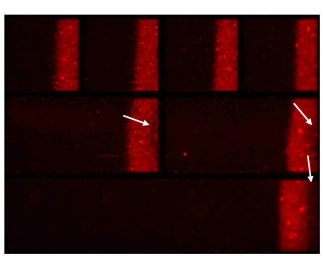

f

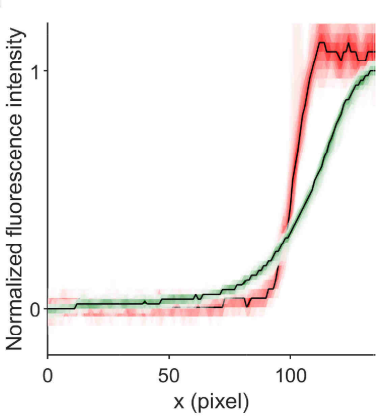

d

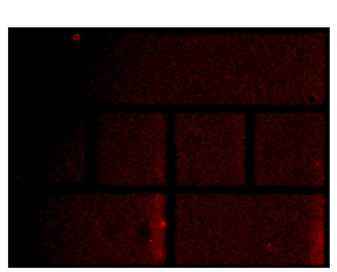

g

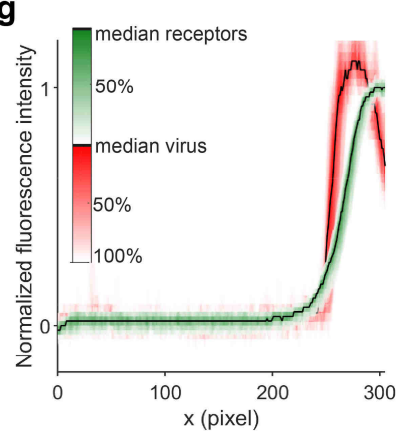

Figure 5.1 | Visualization of superselective binding of IAV. a) Method to form gradients and visualize superselectivity. In SLBs between electrodes inside a microchannel (positions A, B, C), electrophoretic gradients of biotinylated lipids are formed. Fluorescently labelled SAv and 2,6-S(LN) $)_{3}$ are bound onto the biotinylated lipids to form a receptor gradient. Fluorescently labelled influenza virus is added, and the colocalization is imaged with fluorescence microscopy. b) Structure of the biotinylated glycan receptor 2,6-S(LN)3. c) Fluorescent micrographs of receptor (green) and virus (red) gradients on SLBs with $0.5 \%$ biotin-DOPE. White arrows indicate areas where little virus bound despite sufficient SAv density. d) Negative control for virus binding using a 
biotinylated glycan without sialic acid $(\mathrm{LN})_{2}$ as a dummy receptor on SLBs with $0.5 \%$ biotin-DOPE. e) Lateral intensity profiles of SAv (green) and virus (red) fluorescence on SLBs with $0.3 \%$ biotin-DOPE in a $100 \mu \mathrm{m}$ corral with an exponential fit (yellow) through the SAv profile. The black lines show the median along the vertical direction. The variance is given by the red and green contours which indicate the percentage of data points between each contour line and the median. f) Intensity profiles on SLBs with $0.5 \%$ biotin-DOPE in a $100 \mu \mathrm{m}$ corral. g) Intensity profiles on SLBs with $0.5 \%$ biotin-DOPE in a $210 \mu \mathrm{m}$ corral.

Whereas previously gradients were formed at low potential to avoid water splitting, ${ }^{36}$ we formed the biotin-DOPE gradients at $2.0 \mathrm{~V}$ for $30 \mathrm{~min}$ to address a wider range of receptor densities. The formation of bubbles at the electrodes was prevented by passing a solution of ferrocene methanol continuously through the flow cell at a high flow rate while the gradient was formed.

After forming the receptor gradients, fluorescently labelled IAV Puerto Rico/8/34 Mt. Sinai (PR8) was passed over the chip in concentrations of $6.25 \cdot 10^{8}-2.0 \cdot 10^{10}$ virus particles (vp) per $\mathrm{ml}(1.04-33.2 \mathrm{pM})$ in the presence of $200 \mathrm{mM}$ Zanamivir overnight at a flow rate of $1 \mu \mathrm{l} / \mathrm{min}$. When PR8 was imaged over the $2,6-\mathrm{S}(\mathrm{LN})_{3}$ gradients, bright areas could be seen against a low intensity background matching with the relatively brighter and darker areas of SAv (Figure 5.1c). The contrast between bright and dark areas was higher for IAV than for SAv, indicating that the binding was superselective. ${ }^{16}$ As a negative control for IAV binding, glycans without sialic acid $(\mathrm{LN})_{2}$ were used as a dummy receptor. The same surfaces were prepared in a quartz crystal microbalance (QCM) experiment where no virus binding was observed (Chapter 4), showing that the SLBs are highly antifouling against IAV. Here, some binding was observed but only at the highest (LN) ${ }_{2}$ densities (Figure 5.1d). Possibly, PR8 retains some affinity for $(\mathrm{LN})_{2}$.

To investigate the range of receptor densities at which superselective binding may be observed, we formed gradients from SLBs with 0.3 and $0.5 \%$ biotinDOPE and studied the shape of the gradients and the co-localization of virus and receptors with fluorescence microscopy. We used $100 \mu \mathrm{m} \times 100 \mu \mathrm{m}$ corral chips and multi-corral chips that have alternatingly one corral of $430 \mu \mathrm{m}$ long, two corrals of $210 \mu \mathrm{m}$, and four corrals of $100 \mu \mathrm{m}$, each with a width of $100 \mu \mathrm{m}$ (Figure 5.1c). With $0.3 \%$ biotin-DOPE in $100 \mu \mathrm{m}$ corrals, the SAv gradient had an exponential shape in accordance with the shape of the electric field as was 
previously described for lipid gradients (Figure 5.1d). ${ }^{36}$ The SAv gradients were not affected by flow direction. With $0.5 \%$ biotin-DOPE, the SAv fluorescence leveled off (Figure 5.1e). In the $210 \mu \mathrm{m}$ and $430 \mu \mathrm{m}$ corrals, the SAv fluorescence was more sharply divided approaching a sigmoidal rather than exponential shape (Figure 5.1f).

The sigmoidal binding profile of IAV, characteristic of superselectivity, could be seen in all corral lengths for both 0.3 and $0.5 \%$ biotin-DOPE. In some binding studies, we found that the virus bound in lower amounts at the areas of the highest receptor densities in the $210 \mu \mathrm{m}$ corrals and $430 \mu \mathrm{m}$ corrals (Figure $5.1 \mathrm{c}$ and $5.1 \mathrm{~g}$ ), whereas these artefacts occurred rarely in $100 \mu \mathrm{m}$ corrals.

The digital imaging transforms continuous gradients into a finely meshed array of discrete samples. At 10x magnification and a resolution of 1360x1024 pixels, each pixel is $641 \times 641 \mathrm{~nm}$. In binding studies of bacteria, the individual bacteria were counted to quantify their binding. ${ }^{31}$ Here, a visual inspection of the fluorescent micrographs (Figure 5.1c) shows that the fluorescence of the viruses is not clearly separated from neighboring viruses so that fluorescence intensity is more appropriate for quantification than counting the number of viruses. The viruses in this study have an average size of $112 \mathrm{~nm}$ (Chapter 4). Assuming random deposition with a maximum coverage of $85 \%,{ }^{40}$ up to 35 virus particles may bind on the area of one pixel. Each pixel is regarded as an individual measurement of receptor and virus density for the purpose of determining the binding profile and threshold receptor density. These measurements are not strictly independent as any degree of defocus causes a spillover of emitted light into neighboring pixels, which has a smoothing effect on the intensity. A not too high gradient steepness should therefore be chosen, as long as plateau values of virus binding can be determined on both sides of the transition.

To determine the colocalization of virus and receptors, fluorescence micrographs in appropriate colors are acquired at identical position and focus and saved as an image stack. The stacked micrographs are cropped into corrals by aligning a mask, using a custom written MATLAB script (Figure 5.2a). Part of a corral outside the microchannel is selected as background. From the edges of the corrals, a part of the image with a width of 10 pixels is discarded. The local receptor density in each pixel is calculated using: 


$$
\rho_{\mathrm{R}, \mathrm{i}}=\bar{\rho}_{\mathrm{R}} \cdot \frac{I_{\mathrm{i}}-\bar{I}_{\mathrm{bg}}}{\bar{I}-\bar{I}_{\mathrm{bg}}}
$$

with $\bar{\rho}_{\mathrm{R}}$ the average receptor density based on the percentage of biotin-DOPE in the SLB and a lipid footprint of $60 \AA^{2},{ }^{41,42} I_{i}$ the local fluorescence intensity, $\bar{I}_{\text {bg }}$ the average fluorescence intensity of the background, and $\bar{I}$ the average intensity of the corral. The local fluorescence intensities are typically approximately 4-fold lower than average at the minimum and 4-fold higher at the maximum. After the local receptor densities are calculated according to Equation 5.1, these values are used on the $x$-axis in plots of virus intensity vs. receptor density (Figure $5.2 \mathrm{~b}-\mathrm{d}$ ).

The fluorescence intensity of the virus in the corresponding micrograph is normalized between 0 and 1 using:

$$
\theta_{\mathrm{V}, \mathrm{i}}=\frac{I_{\mathrm{i}}-I_{\min }}{I_{\max }-I_{\min }}
$$

with $\theta_{\mathrm{V}, \mathrm{i}}$ the virus coverage, $I_{\mathrm{i}}$ the local fluorescence intensity, $I_{\min }$ the minimum fluorescence intensity, and $I_{\max }$ the maximum fluorescence intensity. Minimum and maximum fluorescence intensities are determined by finding the maxima of $2 \mathrm{D}$ kernel estimations of the data points with the 50 lowest and $10 \%$ highest receptor densities. These values to select the minimum and maximum showed a robust balance between ignoring outliers and finding the extremes in data sets of varying sizes. The normalization of the virus coverage makes the sigmoidal binding profile act as an internal standard that allows the threshold receptor density to be compared across different micrographs regardless of variations in imaging conditions. 

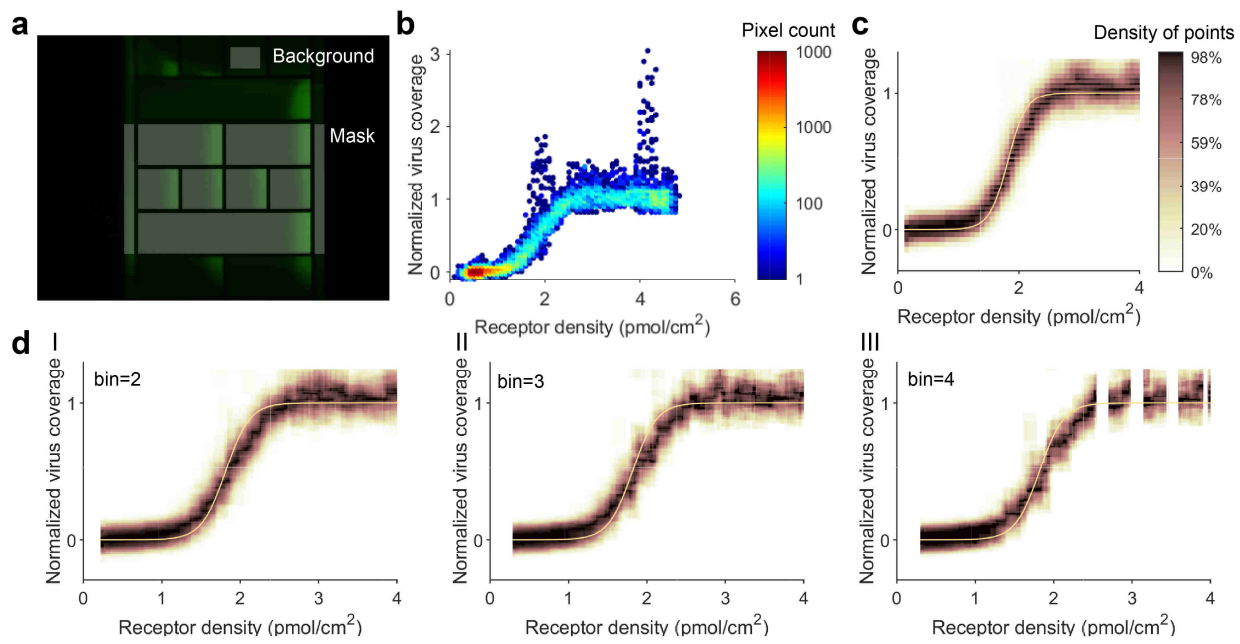

Figure 5.2 I Image processing of fluorescence micrographs to extract IAV binding profiles as function of receptor density. a) Corrals and background are selected from the receptor image with a mask. The same selection is used for the corresponding IAV image. b) "Point cloud" of one corral, showing the normalized virus coverage as function of receptor density. c) Binding profile of one corral, fitted with Equation 5.4. Data points are shown in a density map that was generated with a rolling average of 2001×250 points, a window of 40 points, and a range of 0.1 in the $y$-direction. The fit is based directly on the point cloud and not on the density map. d) Binding profiles and fits where the data was binned into superpixels with a size of $2 \times 2,3 \times 3$, and $4 \times 4$ pixels.

The normalized virus coverage, calculated according to Equation 5.2, is plotted against the receptor density of each pixel in a "point cloud" (Figure 5.2b). The point cloud is unevenly distributed over the $x$-axis because the receptor density increases exponentially over the length of a corral. To visualize the discrete and unevenly distributed point cloud as a binding profile, we used a rolling window average. The average was evaluated at $2001 \times 250$ points with a window of 40 points and a range of 0.1 in the $y$-direction, in which we normalized the number of data points to the maximum within the window to obtain a continuous binding profile where the areas with the highest local density of data points are indicated with a dark color (Figure 5.2c). This binding profile forms a direct visualization of the superselective binding profiles that were predicted by Martinez-Veracoechea and Frenkel. ${ }^{16}$ 
The point cloud, which is a representation of the IAV binding data as a function of receptor density, can be fitted using a theoretical binding model, based on the statistical thermodynamics of multivalent adsorption, ${ }^{26,30}$ that was described earlier in Chapter 4:

$K_{\mathrm{av}}=N_{\mathrm{A}} V_{\mathrm{ex}}\left(1+\frac{K_{\mathrm{i}, \text { eff }}}{N_{\mathrm{A}} V_{\text {explore }}}\right)^{A_{\text {contact }} \sigma_{\mathrm{R}}}$

where $K_{\mathrm{av}}$ is the multivalent equilibrium binding constant, $N_{\mathrm{A}}$ is Avogadro's number, $V_{\mathrm{ex}}$ the excluded volume of a virus particle, $K_{\mathrm{i}, \text { eff }}$ the effective equilibrium constant of an individual HA-glycan interaction, $V_{\text {explore }}$ the volume accessible to a glycan, $A_{\text {contact }}$ the surface area that can be reached by HA when the virus is bound, and $\sigma_{\mathrm{R}}$ the receptor density. The product $A_{\text {contact }} \sigma_{\mathrm{R}}$ is the number of possible simultaneous virus-receptor interactions a virus can form on average. The resulting virus binding profile is described by:

$\theta=\frac{K_{\mathrm{av}}\left(\sigma_{\mathrm{R}}\right)[V]}{1+K_{\mathrm{av}}\left(\sigma_{\mathrm{R}}\right)[V]}$

where $\theta$ is the virus coverage, $K_{\mathrm{av}}\left(\sigma_{\mathrm{R}}\right)$ is the receptor density-dependent $K_{\mathrm{av}}$ (according to Equation 5.3), and [ $V$ the virus concentration. The threshold receptor density is the point where $K_{\mathrm{av}}\left(\sigma_{\mathrm{R}}\right)[V]=1$.

This model is fitted to a point cloud using $\frac{K_{\mathrm{i}, \text { eff }}}{N_{\mathrm{A}} V_{\text {explore }}}$ as fitting parameter. With all other constants given, both the threshold receptor density and the steepness of the fit depend on $\frac{K_{\mathrm{i}, \text { eff }}}{N_{\mathrm{A}} V_{\text {explore }}}$. In Figure $5.2 \mathrm{C}$, the fit is slightly steeper than the experimental binding profile for one corral. Point clouds obtained from multiple corrals in multiple micrographs are combined to obtain a more reliable binding profile and fit.

Because the pixel size is only one order of magnitude larger than the size of a virus, we investigated if binning adjacent pixels into larger superpixels would decrease the noise from stochastically adsorbed viruses (Figure 5.2d). Increasing the bin size progressively decreases the resolution of the binding profile around the threshold receptor density and broadens the confidence interval of the fit. While binning may generally help to suppress noise, in this case it also lowers the accuracy because the underlying properties of adjacent pixels (even above or below) are not necessarily the same and the virus binding 
depends nonlinearly on receptor density. This also suggests that increasing the resolution or color depth of the micrographs would further improve the accuracy of the binding profiles.

Equation 5.4 assumes thermodynamic equilibrium. To test whether this applied, we passed a PR8 solution over the chip at a flow rate of $1 \mu \mathrm{l} / \mathrm{min}$ and acquired fluorescence micrographs at $30 \mathrm{~min}$ intervals. Over time, the fluorescence intensity increased towards a plateau that is not reached within the time of the measurement (Figure 5.3a). The fluorescence intensity increased at the same relative rate at the different glycan densities so that the shape of the binding profile did not change in time (Figure 5.3b). As soon as the signal-to-noise ratio was high enough, the fitted threshold receptor density stabilized so that the variance between corrals decreased (Figure 5.3c). From $360 \mathrm{~min}$ onward, the average threshold receptor density did not differ significantly from that of the last frame, which was long before the fluorescence intensity reached its plateau. When the chip was washed with PBS with and without $200 \mathrm{mM}$ Zanamivir at a flow rate of $10 \mu \mathrm{l} / \mathrm{min}$ for $30 \mathrm{~min}$, the threshold receptor density did not change but the fluorescence intensity dropped slightly (Figure 5.3a). The stability of the threshold receptor density suggests that both the adsorption rate and desorption rate of IAV are not affected by mass transport limitation. 


\section{Chapter 5}

a

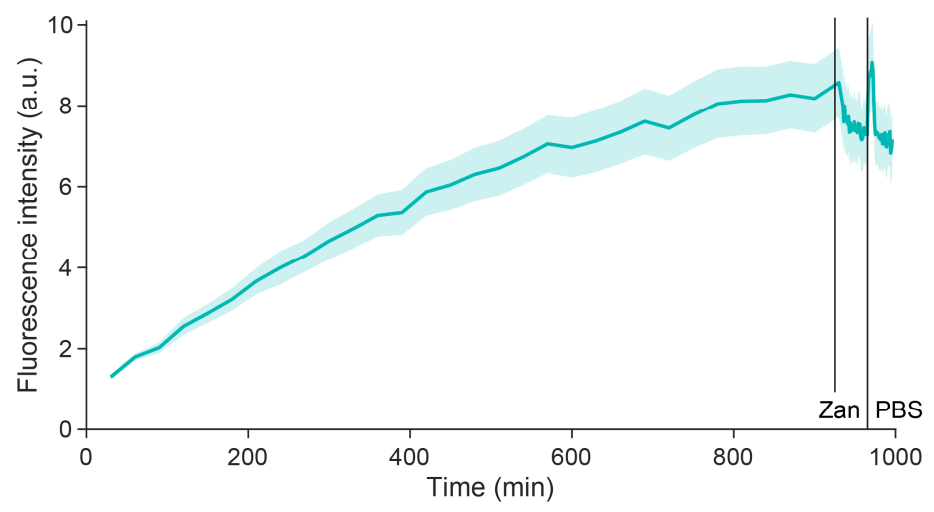

b
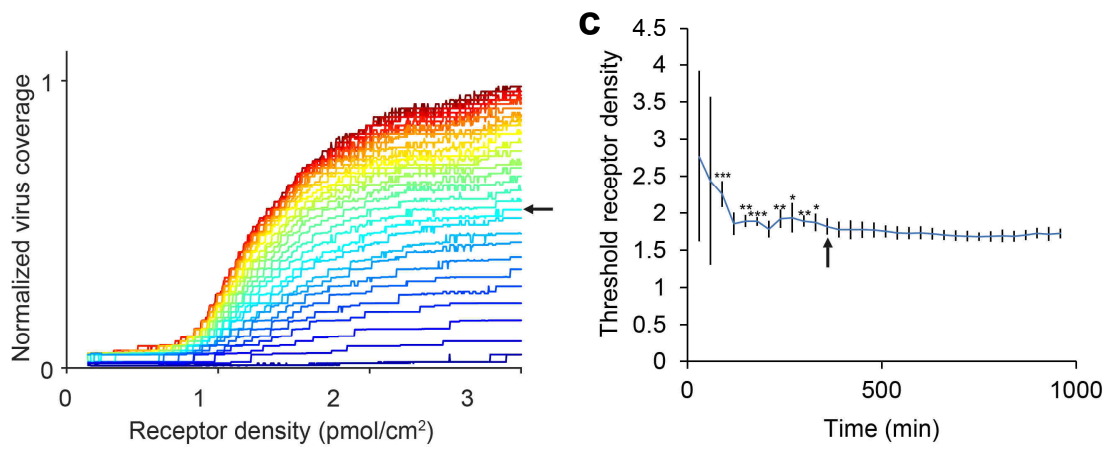

Figure 5.3 | Threshold receptor density as a function of time. a) Development of the average fluorescence intensity over time in $100 \mu \mathrm{m}$ corrals of a mixed corral flow cell with $2,6-\mathrm{S}(\mathrm{LN})_{3}$ gradients on $0.5 \%$ biotin-DOPE. The shaded area indicates the standard error between corrals. After $930 \mathrm{~min}$ of PR8, the solution was changed to $200 \mathrm{mM}$ Zanamivir, the flow rate to $10 \mu \mathrm{l} / \mathrm{min}$ and micrographs were taken every min. After 960 min, the solution was changed to PBS without NA inhibitor. b) Development of virus binding profile over time (from 30-930 min). The time between frames was $30 \mathrm{~min}$. The median fluorescence intensity of the virus was normalized to the highest value in the last frame. c) Development of the threshold receptor density over time. Error bars show the standard deviation between corrals. From 360 min onward, the threshold density does not differ significantly from that of the last frame. This point is indicated with an arrow in both $a$ and b. $*$ : $<0.05,{ }^{* *}: p<0.01,{ }^{* *}: p<0.001$ 
a

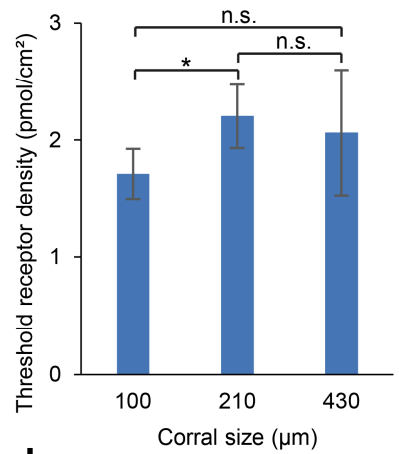

d

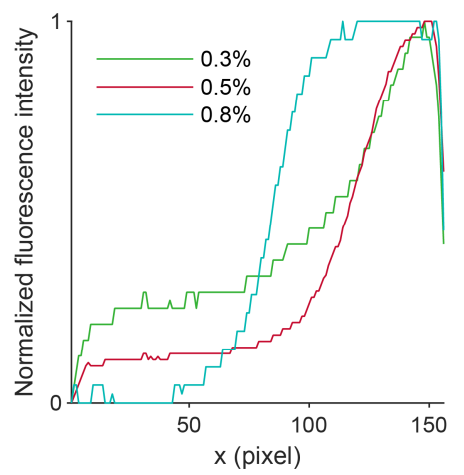

b

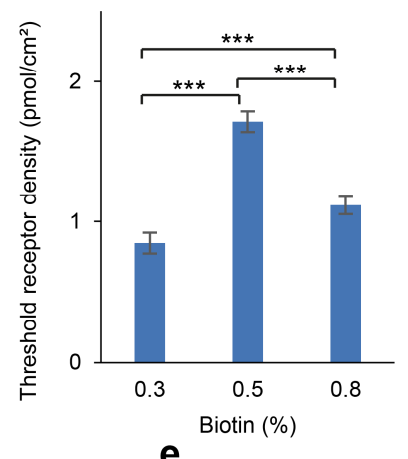

e
C

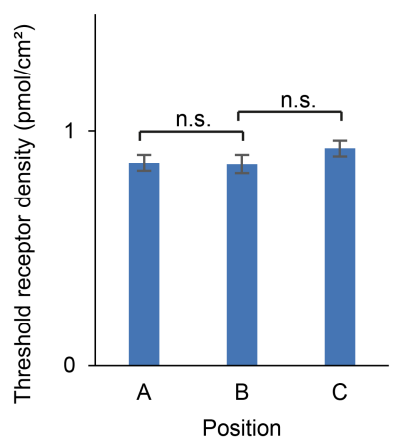

Figure 5.4| Threshold densities observed for different layout and biotin densities. a) Mean threshold receptor density for corrals of different sizes on gradients from $0.5 \%$ biotin-DOPE. Error bars indicate $95 \%$ confidence interval. n.s.: not significant, $*$ : $p<$ 0.05 . b) Mean threshold receptor density on gradients prepared from different percentages of biotin-DOPE in $100 \mu \mathrm{m}$ corrals. Error bars indicate $95 \%$ confidence interval. ${ }^{* * *}: p<0.001$. c) Mean threshold receptor density on $0.3 \%$ biotin-DOPE in 100 $\mu \mathrm{m}$ corrals for different positions in the microchannel (A-C, Figure 5.1a). Gradients in A and $C$ are with the direction of the flow through the channel; $B$ is against the flow. Error bars indicate $95 \%$ confidence interval. n.s.: not significant. d) Median lateral intensity profiles of SAV on gradients prepared from different percentages of biotin-DOPE in a $100 \mu \mathrm{m}$ corral, without the edges removed from the image. e) Median lateral intensity profiles of SAv (green) and ATTO 565-biotin (red) fluorescence on 0.5\% biotin-DOPE in a $100 \mu \mathrm{m}$ corral.

The actual threshold receptor density is expected to be independent from the layout of the receptor gradients. To test whether the layout of the gradients influenced the observed threshold receptor density, we compared the mean 
threshold receptor densities for different corral sizes and different positions in the microchannel, using a two-way ANOVA followed by a multiple comparison test. The threshold receptor density was significantly higher for $210 \mu \mathrm{m}$ corrals than for $100 \mu \mathrm{m}$ corrals $(\mathrm{p}<0.05)$, and the variance within $430 \mu \mathrm{m}$ corrals was high (Figure 5.4a). We compared the observed threshold receptor densities for gradients made from SLBs with different percentages of biotin-DOPE and found that the threshold densities differed strongly $(p \ll 0.001)$ (Figure $5.4 b)$. We found no significant difference $(p>0.05)$ between the three positions in the microchannel or between the two gradient directions, with or against the flow direction (Figure 5.4c). This suggests that the differences in the observed threshold receptor density for different layouts and different percentages of biotin-DOPE do not arise from mass transport limitations.

Figure $5.4 \mathrm{~d}$ shows the lateral intensity profiles of gradients from different percentages of biotin-DOPE. While the gradients from $0.3 \%$ biotin-DOPE have an exponential shape in accordance with the electric field (Figure 5.1d), the $0.5 \%$ gradients level off, and the $0.8 \%$ gradients have a distinct sigmoidal shape. This sigmoidal shape is more strongly pronounced in the longer $210 \mu \mathrm{m}$ and 430 $\mu \mathrm{m}$ corrals (Figure $5.1 \mathrm{~g}$ ). At both ends of the intensity profiles, the fluorescence drops off near the edge of the corrals. The deviation from an exponential shape for higher biotin-DOPE percentages suggests that the maximum density of SAv is reached. In studies with quartz crystal microbalance (QCM), SLBs are typically saturated with SAv at around $1 \%$ biotin-DOPE. ${ }^{41,43}$ This means that the number of biotin groups per SAv is not constant over the whole gradient for higher biotin percentages and $\bar{\rho}_{\mathrm{R}}$ cannot be calculated directly from the percentage of biotin-DOPE in the SLB but is likely overestimated. If Equation 5.1 is then used to calculate the local receptor densities $\rho_{\mathrm{R}, \mathrm{i}}$, the observed threshold receptor density will also be overestimated. As long as the actual threshold receptor density is well below the density where the SLBs are saturated with SAv, this can be avoided by using only $100 \mu \mathrm{m}$ corrals and no more than $0.3 \%$ biotinDOPE in the SLBs.

Dubacheva et al. reported that the residual valency of SAv increases with biotin density in fluid-state SLBs but decreases with biotin density in self-assembled monolayers. ${ }^{41}$ To test whether the residual valency of SAv in the gradients on gel-state SLBs changes, we incubated SAv gradients on $0.5 \%$ biotin-DOPE with 
fluorescent ATTO 565-biotin (Figure 5.4e). The lateral intensity profile of ATTO 565-biotin and SAv are the same, suggesting that the residual valency of SAv in the gradients is constant and no additional constraints on the percentage of biotin-DOPE are necessary.

5.2.5 Effects of virus concentration and flow rate on threshold receptor density

To study the influence of virus concentration and flow rate on the observed threshold receptor density, we measured the virus binding on gradients from $0.3 \%$ biotin-DOPE in $100 \mu \mathrm{m}$ corrals at flow rates of $1 \mu \mathrm{l} / \mathrm{min}$ and $5 \mu \mathrm{l} / \mathrm{min}$ and titrated the virus concentration. We found no significant change in threshold density $(p>0.5)$ with an 8-fold increase in virus concentration (Figure 5.5a) but found a strong difference between $1 \mu \mathrm{l} / \mathrm{min}$ and $5 \mu \mathrm{l} / \mathrm{min}(\mathrm{p} \ll 0.001)$.

From Equations 5.3 and 5.4 it follows that the threshold receptor density depends on virus concentration by:

$\sigma_{\mathrm{R}, \text { threshold }}=\frac{-\ln \left(N_{\mathrm{A}} V_{\mathrm{ex}}[V]\right)}{\ln \left(1+\frac{K_{\mathrm{i}}}{N_{\mathrm{A}} V_{\text {explore }}}\right) A_{\text {contact }}}$

This relationship is plotted in Figure 5.5b over the experimentally determined threshold receptor densities. The difference in $\sigma_{\mathrm{R} \text {,threshold }}$ predicted by Equation 5.5 with a change in virus concentration of one order of magnitude is small. While the experimentally determined $\sigma_{\mathrm{R}, \text { threshold }}$ appears to follow Equation 5.5, the difference in $\sigma_{\mathrm{R} \text {,threshold }}$ between the highest and lowest virus concentrations remains not significant.

While there was a strong difference in threshold receptor density between flow rates of $1 \mu \mathrm{l} / \mathrm{min}$ and $5 \mu \mathrm{l} / \mathrm{min}$, there was no significant difference in threshold density between gradients with or against the flow direction for either flow rate. This suggests that increased shear flow does not disrupt mass transport or "push" the viruses to a different position. Instead, the flow appears to provide a shear force that works on all surface-bound viruses regardless of their position and opposes their interaction with the surface. Additional measurements between $1 \mu \mathrm{l} / \mathrm{min}$ and $5 \mu \mathrm{l} / \mathrm{min}$ indicate a nonlinear trend (Figure $5.5 \mathrm{~d}$ ). 


\section{Chapter 5}

a

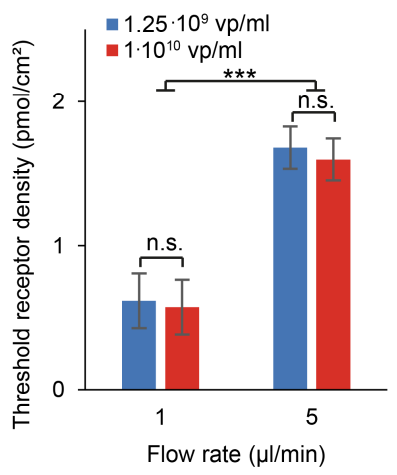

C
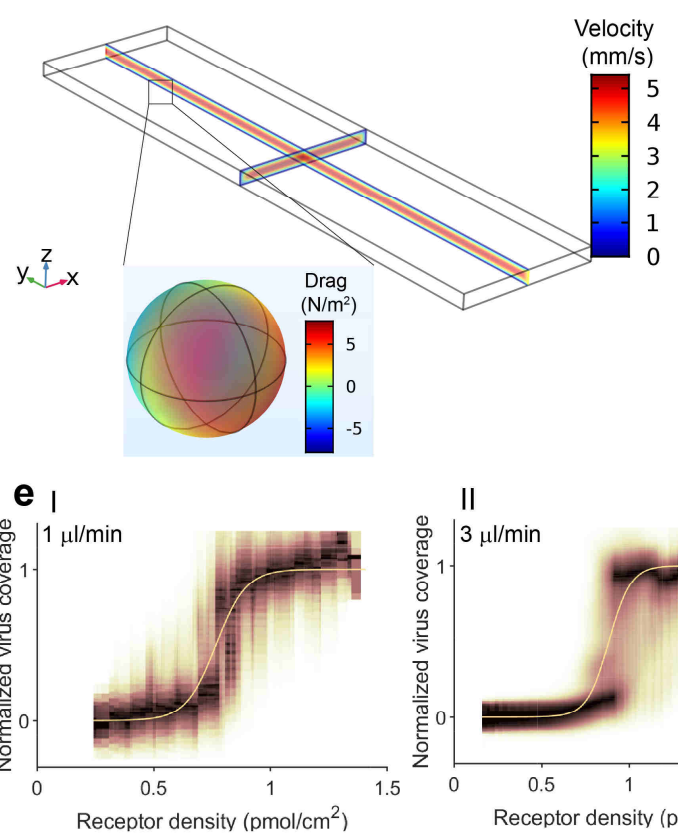

b

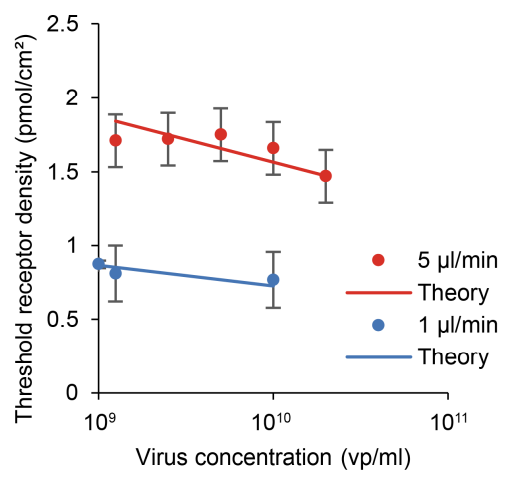

d
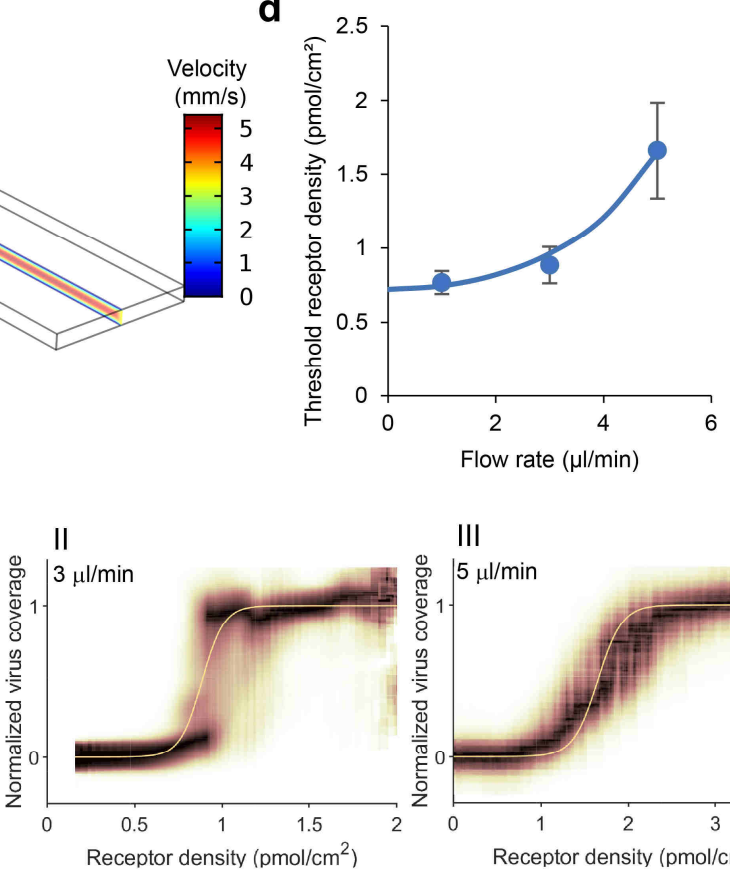

III

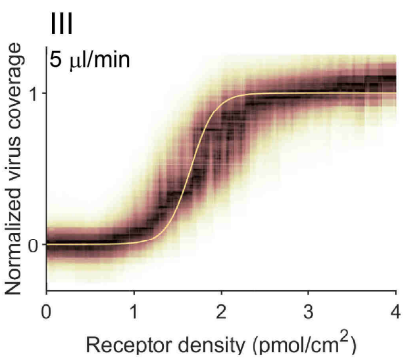

Figure 5.5| Threshold receptor density as a function of flow rate and virus concentration. a) Mean threshold receptor density for different virus concentrations and flow rates. Error bars indicate $95 \%$ confidence interval. n.s.: not significant, ${ }^{* * *}: \mathrm{p}$ $<0.001$. b) Measured and predicted threshold receptor density at different virus concentrations for different flow rates. The theoretical change in threshold receptor density with virus concentration is calculated separately for $1 \mu \mathrm{l} / \mathrm{min}$ and $5 \mu \mathrm{l} / \mathrm{min}$ using Equation 5.5 with $\frac{K_{\mathrm{i}}}{N_{\mathrm{A}} V_{\text {explore }}}$ as fit parameter. Error bars indicate $95 \%$ confidence 
interval. c) Finite element simulation of laminar flow in a microchannel at a flow rate of $5 \mathrm{\mu l} / \mathrm{min}$ and the resulting drag force on a $112 \mathrm{~nm}$ particle. d) Fitted threshold receptor density at different flow rates using Equation 5.6 with the spring constant $k$ as fit parameter. Error bars indicate standard deviation. e) Binding profiles and fits at flow rates of $1 \mu \mathrm{l} / \mathrm{min}, 3 \mu \mathrm{l} / \mathrm{min}$, and $5 \mu \mathrm{l} / \mathrm{min}$.

Curk and Tito described a theoretical model for multivalent particles that bind superselectively to a surface under a force that opposes binding. ${ }^{18}$ If a pulling force works on a bound particle that is not strong enough to break all interactions between the particle and the surface at the threshold receptor density, the threshold receptor density is shifted and superselectivity is enhanced. The shift depends on the strength of the force by:

$\ln \left(c_{\mathrm{R}, \mathrm{eff}}^{o}\right) \propto \ln \left(c_{\mathrm{R}}^{o}\right)+\frac{1}{2 k R T}\left(\frac{F_{\mathrm{pull}}}{N_{\mathrm{L}}}\right)^{2}$

where $c_{\mathrm{R}, \text { eff }}{ }=\sigma_{\mathrm{R} \text {,threshold }} N_{\mathrm{A}}$ is the shifted intrinsic transition point under the applied force, $c^{\circ}$ the intrinsic transition point without applied force, $k$ the spring constant, $R$ the ideal gas constant, $T$ the absolute temperature, $F_{\text {pull }}$ the pulling force, and $N_{\mathrm{L}}$ the number of participating ligands. $F_{\text {pull }}$ acts perpendicularly to the surface, so this theory may not be accurate for the shear force directly.

We used a finite element simulation in COMSOL to estimate $F_{\text {pull. }}$. We simulated a laminar flow of water at $1 \mu \mathrm{l} / \mathrm{min}$ and $5 \mu \mathrm{l} / \mathrm{min}$ through a $2000 \mu \mathrm{m} \times 500 \mu \mathrm{m}$ $x 50 \mu \mathrm{m}$ channel with a $112 \mathrm{~nm}$ sphere at the bottom surface in the middle of the channel at $0.8 x$ the channel length and computed the flow velocity in the channel and the drag force on the sphere (Figure 5.5c). At a flow rate of 5 $\mu \mathrm{l} / \mathrm{min}$, the drag force was $42.5 \mathrm{fN}$, which is 10 -fold higher than the product of the virus cross-section and the shear force that was reported previously for a simulation of a microchannel of the same cross-section. ${ }^{31}$ The simulated drag force is much lower than the rupture force of a single HA-sialic acid interaction in AFM. ${ }^{8}$ We fitted $k$ to obtain the relationship shown in Figure $5.5 \mathrm{~d}$, which fits the data qualitatively, but found $k / R T=2.21 \cdot 10^{-15} \mathrm{~nm}^{-2}$, which is extremely low.

It is probable that the actual drag force near the wall is higher due to local interaction with the no-slip wall. Nir et al. described an analytical model that considers the interaction of a nanoparticle tethered to a wall by a DNA strand: 
$F=\frac{k_{\mathrm{B}} T}{D_{0} f} \dot{y} \delta z$

where $F$ is the hydrodynamic force, $k_{\mathrm{B}}$ the Boltzmann constant, $D_{0}$ the diffusion coefficient of the particle, $f$ the Faxén correction factor which is a function of the particle radius and its distance from the wall, $\dot{y}$ the shear rate, and $\delta z$ the length of the transverse fluctuations of the particle. ${ }^{44}$ From the molecular dynamics simulations described in Chapter 4 , the minimum and maximum length of $2,6-\mathrm{S}(\mathrm{LN})_{3}$ are $2.9 \mathrm{~nm}$ and $4.5 \mathrm{~nm}$. We estimate therefore that $2.9 \mathrm{~nm}$ is the minimum distance of the virus from the wall and $\delta z=1.6 \mathrm{~nm}$. This would give a Faxén correction factor of $f=0.33$, a hydrodynamic force of $F=130 \mathrm{fN}$ at a flow rate of $5 \mu \mathrm{l} / \mathrm{min}$, and a spring constant of $k / R T=2.07 \cdot 10^{-14} \mathrm{~nm}^{-2}$.

Contrary to the enhanced superselectivity that was predicted by Curk and Tito in the case of a perpendicular pulling force, ${ }^{18}$ the experimental binding profiles as function of receptor density under shear force show a less steep transition around the threshold density at higher flow rate (Figure 5.5e). Together with the extremely low spring constant that is fitted with Equation 5.6, this suggests that the model does not fully apply for forces parallel to the surface in its current form, even though the experimental data shows the predicted increase in threshold receptor density with applied force. A deeper theoretical effort would need to be invested to examine how the theory changes when particles are under shear flow as opposed to surface-normal force. Similarly, the length and flexibility of receptors and ligands are expected to influence the theory. The present model also ignores the kinetics of forming multiple interactions under a shear force. Although the kinetics of forming multiple interactions have been reported to be relatively fast, ${ }^{19}$ it is possible that fewer simultaneous interactions are possible than Equation 5.6 assumes. Molecular dynamics simulations may form a valuable tool in adjusting the model for the case of influenza virus under shear flow.

The flow rates of 1-5 $\mu \mathrm{l} / \mathrm{min}$ that were addressed in this study correspond to wall shear stresses of 0.9-4.3 dynes $/ \mathrm{cm}^{2},{ }^{31}$ which are within the range of those a virus may encounter in vivo. In healthy blood vessels, the wall shear stress ranges from 0.8-60 dynes/ $\mathrm{cm}^{2} .{ }^{45} \mathrm{In}$ the airways and nasal cavity, the wall shear stress ranges from 0.5-3 dynes/ $\mathrm{cm}^{2}$ during regular breathing and up to 1700 dynes $/ \mathrm{cm}^{2}$ during coughing. ${ }^{46,47}$ In the periciliary layer, in which beating cilia 
propel the mucus above it, wall shear stresses around 0.08 dynes $/ \mathrm{cm}^{2}$ are estimated. ${ }^{48}$ The strong effect of variations in shear force on the threshold receptor density of IAV may play an important role in virus tropism. The decreased avidity of the virus under high shear force may facilitate airborne transmission via sneezing and coughing.

\subsection{Conclusions}

Receptor gradients over $100 \mu \mathrm{m}$ corrals covered a range of receptor densities that was sufficient to visualize superselective binding of PR8, and a method of analysis is shown that can exploit the multiplicity in the chip for accurate determination of the threshold receptor density. A layout of different corral lengths may be useful for efficient optimization but offers fewer data points around the threshold receptor density. For larger particles, such as bacteria, the larger corrals may be favorable. ${ }^{31}$ Lowering the potential during gradient formation may increase the number of data points around the threshold as long as sufficient data points in the lower and higher plateau can be taken for normalization.

The threshold receptor density is constant over time. When the gradient formation leads to biotin densities at the high end beyond what is necessary to saturate the surface with $S A v$, the average receptor density is overestimated and thereby the threshold receptor density as well. The effect of virus concentration on the threshold receptor density is very small. When a shear force is exerted on bound viruses, the threshold receptor density is increased.

The MAP chip allows direct visualization of superselective binding, which not only gives a clearer insight in superselectivity but also makes it a more efficient platform for the quantitative assessment of multivalent interactions than platforms that probe one receptor density at a time, such as BLI. This flow cell can also be a powerful experimental tool in further elucidating the relationship between superselectivity and hydrodynamic forces, which may offer insights into the airborne transmission of pathogens as well as tools to optimize the targeted delivery of drugs that are distributed via the blood circulation. 


\subsection{Acknowledgements}

Erik Hamming is thanked for his role in the image analysis and the fitting of the theoretical models to experimental data. Nicholas Tito is thanked for his help with the theoretical models. Malte Tieke and Erhard van der Vries are thanked for the production and characterization of the viruses. Zeshi Li and Geert-Jan Boons are thanked for the synthesis of the glycans. Wouter Vijselaar is thanked for the cleanroom fabrication of the gradient chips. Annemarie Huijser and Willem Vos are thanked for discussions with regards to the fluorescence quantification. Nicole Zeijen and Bea Overeem-Quist are thanked for their help with statistics.

\subsection{Experimental}

\subsubsection{Materials}

Biotinylated glycans $2,6-\mathrm{S}(\mathrm{LN})_{3}$ and $(\mathrm{LN})_{2}$ were provided by Zeshi Li, and GeertJan Boons, and synthesized as described in Chapter 4. All other starting materials and chemicals were purchased from Sigma-Aldrich, Fluka, Serva, Becton Dickinson, Avanti Polar Lipids, Acros organics, Microchem, Biotium, Thermo Fisher, Lonza, Schott and Fujifilm, and they were used as received, unless otherwise stated.

\subsubsection{Virus stock preparation}

Influenza A/Puerto Rico/8/34 virus (Mt. Sinai strain) stocks were prepared by propagating the virus in 10-days-old embryonated chicken eggs (GD Animal health, Deventer, The Netherlands) at $33{ }^{\circ} \mathrm{C}$. Allantoic fluids were harvested after 48-72 $\mathrm{h}$ and cleared from debris by centrifugation at 3,000 rpm for $10 \mathrm{~min}$ at $4{ }^{\circ} \mathrm{C}$. Subsequently, the viruses were pelleted by centrifugation at $7,000 \mathrm{rpm}$ for $18 \mathrm{~h}$ at $4{ }^{\circ} \mathrm{C}$ and resuspended in PBS (pH 7.4) (Lonza). Viruses were then purified further by loading of the virus sample on a discontinuous sucrose gradient $(10-50 \% \mathrm{w} / \mathrm{v})$ and centrifugation for $45 \mathrm{~min}$ at 25,000 rpm using a SW41 swing-out rotor. The virus-containing sucrose layer was finally harvested and dialyzed (Slide-A-Lyzer, Thermo Scientific) for $48 \mathrm{~h}$ at $4{ }^{\circ} \mathrm{C}$ to remove the remaining sucrose. Finally, the virus particle count and size distribution were determined using a NS300 nanoparticle tracking analyzer (Malvern, Nanosight), and diluted to a final stock concentration of $1 \times 10^{11}$ particles $/ \mathrm{ml}$, UV-inactivated $\left(50 \mathrm{~mJ} / \mathrm{cm}^{2}\right.$, at a wavelength of $365 \mathrm{~nm}$ ) and aliquots were frozen at $-80{ }^{\circ} \mathrm{C}$. 
Inactivation was confirmed by growing of these viruses on Madin-Darby canine kidney (MDCK) cells. The receptor binding capacity of the viruses was confirmed with a hemagglutination assay that was performed as described elsewhere ${ }^{49}$ to obtain a titer of 2048.

\subsubsection{Virus labelling}

Influenza viruses were fluorescently labelled using the lipophilic dye octadecyl rhodamine B (R18; Thermo Scientific), which binds the virus membrane with the fluorophore at the aqueous interface. R18 dye was mixed with inactivated virus stocks to a final concentration of $80 \mathrm{nM}$ and incubated in the dark for $2 \mathrm{~h}$ on ice. Free dye was subsequently separated from the R18-labelled viruses by adding $25 \mu \mathrm{l}$ Capto core 700 virus purification beads (GE Healthcare) per $1 \mathrm{ml}$ of virus and incubating by rotating the mixture for $30 \mathrm{~min}$ at $4^{\circ} \mathrm{C}$. Finally, the beads were cleared from the virus-containing supernatant by pelleting of the beads by centrifugation at $1,200 \mathrm{rpm}$ for $10 \mathrm{~min}$ in a table centrifuge at $4^{\circ} \mathrm{C}$.

\subsubsection{Chip fabrication}

Flow cells were fabricated according to the procedure described earlier in Chapter 3. A bilayer lift-off recipe was used for fabricating Au electrodes on Mempax glass wafers (Schott). First, LOR 5A (MicroChem) was spin-coated, after which normal lithography was performed on top with Olin OiR 907-17 photoresist (FujiFilm) to create a bilayer resist stack. Electrode patterns were made by exposing the photoresist through a patterned photomask and developing in Olin OPD 4262 (Fujifilm). The develop step washed away the exposed photoresist, and etching through the LOR $5 \mathrm{~A}$ layer created an undercut. Then, $5 \mathrm{~nm}$ Ti and $95 \mathrm{~nm}$ Au were deposited via e-beam evaporation (BAK 600, Balzers). The bilayer resist was then removed by sonication in acetone (20 $\mathrm{min}$ ) and isopropanol (10 $\mathrm{min}$ ) followed by $5 \mathrm{~min}$ immersion in OPD 4262, serving as a sacrificial layer to leave patterned Au electrodes on Mempax glass. To fabricate the $\mathrm{Cr}$ corrals ( $10 \mathrm{~nm}$ thick) in between the Au electrodes, the same procedure was performed a second time, but in this case following alignment with respect to the Au electrodes. 


\subsubsection{PDMS flow channel}

Silicon flow channel masters were produced by standard photolithography steps and deep reactive ion etching. The polydimethylsiloxane (PDMS) flow channels were prepared from a degassed mixture of 10:1 Sylgard 184 elastomer and curing agent (Dow Corning Corp), which was cast onto the silicon master and cured at $60{ }^{\circ} \mathrm{C}$ overnight. The flow channels were cut to size and inlets and outlets were punched using a $1 \mathrm{~mm} \varnothing$ punch (Harris Uni-core, Sigma-Aldrich). After bonding to the chip, a flow channel of $6,000 \times 500 \times 50 \mu \mathrm{m}$ was prepared with a second channel entering from the side.

\subsubsection{PDMS bonding}

Chips were rinsed and sonicated extensively with acetone, ethanol and MilliQ water, and dried prior to UV-ozone exposure (UV/Ozone Procleaner plus, Bioforce Nanosciences) for at least $20 \mathrm{~min}$. After UV exposure, the chips were rinsed with ethanol and water, and dried under a stream of nitrogen. Both cutout PDMS flow channels and cleaned chips were treated with oxygen plasma for $30 \mathrm{~s}$ at $40 \mathrm{~W}$ (Plasma prep II, SPI supplies) after which they were bonded immediately. The chips were placed on a hot plate for $10 \mathrm{~min}$ at $70{ }^{\circ} \mathrm{C}$ to increase the binding strength. Tygon Microbore tubing S- $54-\mathrm{HL}$ (VWR, $0.25 \mathrm{~mm}$ inner $\varnothing$ and $0.75 \mathrm{~mm}$ outer $\varnothing$ ) of $80 \mathrm{~cm}$ for the inlets and $40 \mathrm{~cm}$ for the outlets was inserted into the PDMS. The assembled flow cell was placed in an oven at $60{ }^{\circ} \mathrm{C}$ for $1 \mathrm{~h}$. Leak-free operation was shown for flow rates up to $200 \mu \mathrm{L} / \mathrm{min}$.

\subsubsection{Lipid vesicle preparation}

MPPC (1-myristoyl-2-palmitoyl-sn-glycero-3-phosphocholine, Avanti) was stored as a $10 \mathrm{mg} / \mathrm{ml}$ stock solution in chloroform at $-20^{\circ} \mathrm{C}$. Biotin-cap-DOPE (1,2-dioleoyl-sn-glycero-3-phosphoethanolamine- $\mathrm{N}$-(cap biotinyl), Avanti) was stored as $0.2 \mathrm{mg} / \mathrm{ml}$ solution. Desired molar ratios were mixed in a glass vial, dried under a flow of nitrogen, and kept under vacuum for at least $1 \mathrm{~h}$. The resulting film was resuspended by vortexing in MilliQ water at $50{ }^{\circ} \mathrm{C}$ to form multilamellar vesicles (MLVs) at $1 \mathrm{mg} / \mathrm{ml}$. The MLV solution was extruded 11 times through a $100 \mathrm{~nm}$ polycarbonate membrane (Avanti) at $50{ }^{\circ} \mathrm{C}$ MPPC. The resulting large unilamellar vesicles (LUVs) were kept at room temperature and used within one week. 


\subsubsection{SLB formation and functionalization}

Before SLB formation, the flow cells were washed with 2\% SDS (Sigma-Aldrich), then rinsed with MilliQ, and washed overnight with $2 \%$ Hellmanex $^{\mathrm{TM}}$ to activate the glass surface. Flow cells were mounted onto a heating plate using Scotch ${ }^{\mathrm{TM}}$ tape and rinsed with MilliQ and PBS, while heating to $50^{\circ} \mathrm{C}$. Shortly before LUV incubation, the LUV solution was diluted to $0.5 \mathrm{mg} / \mathrm{ml}$ with PBS. The diluted LUV solution was passed through the flow cells through the secondary inlet for 30 $\min (10 \mu \mathrm{L} / \mathrm{min}$; primary inlet $1 \mu \mathrm{L} / \mathrm{min}$ PBS) to allow adsorption and rupture of the vesicles on the chips. The chips were then washed with MilliQ $(100 \mu \mathrm{L} / \mathrm{min}$ secondary inlet, $10 \mu \mathrm{L} / \mathrm{min}$ primary inlet), after which freshly prepared $0.5 \mathrm{mM}$ hydroxymethylferrocene ( $\mathrm{FcMeOH}, \mathrm{Acros})$ was passed through the device (50 $\mu \mathrm{L} / \mathrm{min}$, both inlets). A potential difference of $2.0 \mathrm{~V}$ was applied over the device for $30 \mathrm{~min}$ to induce electrophoresis in the SLBs. Subsequently, the chips were cooled rapidly on a heat exchanger to fix the surface gradient. The flow cells were then rinsed with MilliQ. Bovine serum albumin (Sigma) $50 \mathrm{mg} / \mathrm{ml}(0.5 \mathrm{ml}$, $10 \mu \mathrm{L} / \mathrm{min}$ ) was passed through the flow cells to form an antifouling layer on the tubing. The flow cells were rinsed with PBS. SAv with Alexa Fluor 488 label (Thermo Fischer) $20 \mu \mathrm{g} / \mathrm{ml}$ was passed through the secondary inlet $(10 \mu \mathrm{L} / \mathrm{min}$, primary inlet $1 \mu \mathrm{L} / \mathrm{min}$ ). The flow cells were rinsed with PBS. The SAv-modified SLBs were inspected with fluorescence microscopy on an Olympus inverted IX71 epi-fluorescence research microscope with X-cite 120PC mercury vapor lamp as light source and a digital Olympus DR70 camera for image acquisition. For Alexa Fluor 488 , blue excitation $\left(460 \leq \lambda_{\mathrm{ex}} \leq 490 \mathrm{~nm}\right)$ and green emission $\left(505 \leq \lambda_{\mathrm{em}} \leq\right.$ $545 \mathrm{~nm}$ ) was filtered using the U-MWG2 Olympus filter cube. Glycan 2,6-S(LN) $3_{3^{-}}$ biotin $500 \mathrm{nM}$ solutions were passed through the primary inlet $(10 \mu \mathrm{L} / \mathrm{min}$, secondary inlet $1 \mu \mathrm{L} / \mathrm{min}$ ), after which the flow cells were rinsed with PBS. During all steps, care was taken to ensure that no air bubbles entered the flow cell.

\subsubsection{Binding studies}

Solutions of IAV Puerto Rico/8/1934 mt. Sinai with R18 label were prepared in PBS with $200 \mu \mathrm{M}$ Zanamivir (GlaxoSmithKline). The virus solution in the desired concentration was passed through the primary inlet of the flow cells at the desired flow rate with the secondary inlet PBS at $10 \%$ of the flow rate through the primary inlet. The bound virus was imaged with fluorescence microscopy. 
For R18, green excitation $\left(510 \leq \lambda_{\mathrm{ex}} \leq 550 \mathrm{~nm}\right)$ and red emission $\left(\lambda_{\mathrm{em}}>590 \mathrm{~nm}\right)$ was filtered.

\subsubsection{Image analysis}

8-bit RGB fluorescence micrographs were aligned and cropped into corrals using a custom written MATLAB program. The fluorescence intensities of receptor and virus labels in matching pixels were listed. The receptor density and virus coverage in each pixel were calculated using Equations 5.1 and 5.2. Binding profiles were obtained by fitting Equation 5.4 with $K_{\mathrm{i}} / N_{\mathrm{A}} V_{\text {explore }}$ as fitting parameter, using the built-in fit function.

\subsubsection{Statistical comparisons}

To determine the confidence interval of threshold receptor densities, point clouds from each corral were fitted individually. Corrals where no gradient had formed or where minimum and maximum virus binding could not be determined were omitted from analysis. Mean and standard deviation $95 \%$ confidence intervals for each group were determined for the fitted threshold receptor densities of all corrals in the group. To determine the value of the threshold receptor density in a group, point clouds from multiple corrals, from multiple positions in the microchannel and multiple experiments were combined into a single larger point cloud to which the fitting was performed. This afforded a better fit than the average of each corral. The $95 \%$ confidence interval of the fit was without exception smaller than the $95 \%$ confidence interval over the threshold densities from all corrals in the group. Unless otherwise specified, the threshold receptor densities shown have been obtained from the combined point cloud with the $95 \%$ confidence interval over all corrals in the group. The significance of the differences in threshold receptor densities was determined with an analysis of variance followed by a multiple comparison test using the MATLAB functions anovan and multcompare.

\subsection{References}

1. Petrova, V. N. \& Russell, C. A. The evolution of seasonal influenza viruses. Nat. Rev. Microbiol. 16, 47-60 (2018).

2. Krammer, F., Smith, G. J. D., Fouchier, R. A. M., Peiris, M., Kedzierska, K., Doherty, P. C., Palese, P., Shaw, M. L., Treanor, J., Webster, R. G. \& García-Sastre, A. Influenza. Nat. Rev. Dis. Prim. 4, 3 (2018).

3. Benton, D. J., Martin, S. R., Wharton, S. A. \& McCauley, J. W. Biophysical measurement 
of the balance of influenza A hemagglutinin and neuraminidase activities. J. Biol. Chem. 290, 6516-6521 (2015).

4. Paulson, J. C. \& de Vries, R. P. H5N1 receptor specificity as a factor in pandemic risk. Virus Res. 178, 99-113 (2013).

5. Zhang, W., Shi, Y., Lu, X., Shu, Y., Qi, J. \& Gao, G. F. An Airborne Transmissible Avian Influenza H5 Hemagglutinin Seen at the Atomic Level. Science 340, 1463-1467 (2013).

6. Di lorio, D., Verheijden, M. L., van der Vries, E., Jonkheijm, P. \& Huskens, J. Weak Multivalent Binding of Influenza Hemagglutinin Nanoparticles at a SialoglycanFunctionalized Supported Lipid Bilayer. ACS Nano 13, 3413-3423 (2019).

7. Xiong, X., Coombs, P. J., Martin, S. R., Liu, J., Xiao, H., McCauley, J. W., Locher, K., Walker, P. A., Collins, P. J., Kawaoka, Y., Skehel, J. J. \& Gamblin, S. J. Receptor binding by a ferrettransmissible H5 avian influenza virus. Nature 497, 392-396 (2013).

8. Cuellar-Camacho, J. L., Bhatia, S., Reiter-Scherer, V., Lauster, D., Liese, S., Rabe, J. P., Herrmann, A. \& Haag, R. Quantification of Multivalent Interactions between Sialic Acid and Influenza A Virus Spike Proteins by Single-Molecule Force Spectroscopy. J. Am. Chem. Soc. 142, 12181-12192 (2020).

9. Reiter-Scherer, V., Cuellar-Camacho, J. L., Bhatia, S., Haag, R., Herrmann, A., Lauster, D. \& Rabe, J. P. Force Spectroscopy Shows Dynamic Binding of Influenza Hemagglutinin and Neuraminidase to Sialic Acid. Biophys. J. 116, 1037-1048 (2019).

10. Hamming, P. H., Overeem, N. J. \& Huskens, J. Influenza as a molecular walker. Chem. Sci. 11, 27-36 (2020).

11. Vahey, M. D. \& Fletcher, D. A. Influenza A virus surface proteins are organized to help penetrate host mucus. elife 8, 1-24 (2019).

12. Guo, H., Rabouw, H., Slomp, A., Dai, M., van der Vegt, F., van Lent, J. W. M., McBride, R., Paulson, J. C., de Groot, R. J., van Kuppeveld, F. J. M., de Vries, E. \& de Haan, C. A. M. Kinetic analysis of the influenza $A$ virus HA/NA balance reveals contribution of NA to virus-receptor binding and NA-dependent rolling on receptor-containing surfaces. PLOS Pathog. 14, e1007233 (2018).

13. de Graaf, M. \& Fouchier, R. A. M. Role of receptor binding specificity in influenza A virus transmission and pathogenesis. EMBO J. 33, 823-841 (2014).

14. Yamada, S., Suzuki, Y., Suzuki, T., Le, M. Q., Nidom, C. A., Sakai-Tagawa, Y., Muramoto, Y., Ito, M., Kiso, M., Horimoto, T., Shinya, K., Sawada, T., Kiso, M., Usui, T., Murata, T., Lin, Y., Hay, A., Haire, L. F., Stevens, D. J., Russell, R. J., Gamblin, S. J., Skehel, J. J. \& Kawaoka, Y. Haemagglutinin mutations responsible for the binding of H5N1 influenza A viruses to human-type receptors. Nature 444, 378-382 (2006).

15. Müller, M., Lauster, D., Wildenauer, H. H. K., Herrmann, A. \& Block, S. Mobility-Based Quantification of Multivalent Virus-Receptor Interactions: New Insights into Influenza A Virus Binding Mode. Nano Lett. 19, 1875-1882 (2019).

16. Martinez-Veracoechea, F. J. \& Frenkel, D. Designing super selectivity in multivalent nanoparticle binding. Proc. Natl. Acad. Sci. U.S.A. 108, 10963-10968 (2011).

17. Dubacheva, G. V., Curk, T., Mognetti, B. M., Auzély-Velty, R., Frenkel, D. \& Richter, R. P. Superselective Targeting Using Multivalent Polymers. J. Am. Chem. Soc. 136, 1722-1725 


\section{Chapter 5}

(2014).

18. Curk, T. \& Tito, N. B. First-order 'hyper-selective' binding transition of multivalent particles under force. J. Phys. Condens. Matter 32, 214002 (2020).

19. Scheepers, M. R. W., van IJzendoorn, L. J. \& Prins, M. W. J. Multivalent weak interactions enhance selectivity of interparticle binding. Proc. Natl. Acad. Sci. U.S.A. 117, 2269022697 (2020).

20. Vachieri, S. G., Xiong, X., Collins, P. J., Walker, P. a, Martin, S. R., Haire, L. F., Zhang, Y., McCauley, J. W., Gamblin, S. J. \& Skehel, J. J. Receptor binding by H10 influenza viruses. Nature 511, 475-477 (2014).

21. Smith, D. F. \& Cummings, R. D. Investigating virus-glycan interactions using glycan microarrays. Curr. Opin. Virol. 7, 79-87 (2014).

22. McQuillan, A. M., Byrd-Leotis, L., Heimburg-Molinaro, J. \& Cummings, R. D. Natural and Synthetic Sialylated Glycan Microarrays and Their Applications. Front. Mol. Biosci. 6, 88 (2019).

23. Stevens, J., Blixt, O., Tumpey, T. M., Taubenberger, J. K., Paulson, J. C. \& Wilson, I. A. Structure and Receptor Specificity of the Hemagglutinin from an H5N1 Influenza Virus. Science 312, 404-410 (2006).

24. Stevens, J., Blixt, O., Glaser, L., Taubenberger, J. K., Palese, P., Paulson, J. C. \& Wilson, I. A. Glycan microarray analysis of the hemagglutinins from modern and pandemic influenza viruses reveals different receptor specificities. J. Mol. Biol. 355, 1143-1155 (2006).

25. Blixt, O., Head, S., Mondala, T., Scanlan, C., Huflejt, M. E., Alvarez, R., Bryan, M. C., Fazio, F., Calarese, D., Stevens, J., Razi, N., Stevens, D. J., Skehel, J. J., van Die, I., Burton, D. R., Wilson, I. a, Cummings, R., Bovin, N., Wong, C.-H. \& Paulson, J. C. Printed covalent glycan array for ligand profiling of diverse glycan binding proteins. Proc. Natl. Acad. Sci. U.S.A. 101, 17033-17038 (2004).

26. Yen, H.-L., Liang, C.-H., Wu, C.-Y., Forrest, H. L., Ferguson, A., Choy, K.-T., Jones, J., Wong, D. D.-Y., Cheung, P. P.-H., Hsu, C.-H., Li, O. T., Yuen, K. M., Chan, R. W. Y., Poon, L. L. M. M., Chan, M. C. W. W., Nicholls, J. M., Krauss, S., Wong, C.-H., Guan, Y., Webster, R. G., Webby, R. J. \& Peiris, M. Hemagglutinin-neuraminidase balance confers respiratorydroplet transmissibility of the pandemic H1N1 influenza virus in ferrets. Proc. Natl. Acad. Sci. U.S.A. 108, 14264-14269 (2011).

27. van Dover, R. B., Schneemeyer, L. F. \& Fleming, R. M. Discovery of a useful thin-film dielectric using a composition-spread approach. Nature 392, 162-164 (1998).

28. Suresh, S. Graded Materials for Resistance to Contact Deformation and Damage. Science 292, 2447-2451 (2001).

29. Fisher, S. A., Tam, R. Y., Fokina, A., Mahmoodi, M. M., Distefano, M. D. \& Shoichet, M. S. Photo-immobilized EGF chemical gradients differentially impact breast cancer cell invasion and drug response in defined 3D hydrogels. Biomaterials 178, 751-766 (2018).

30. Tanes, M. L., Xue, J. \& Xia, Y. A general strategy for generating gradients of bioactive proteins on electrospun nanofiber mats by masking with bovine serum albumin. $J$. Mater. Chem. B 5, 5580-5587 (2017). 
31. Van Weerd, J., Sankaran, S., Roling, O., Sukas, S., Krabbenborg, S., Huskens, J., le Gac, S., Ravoo, B. J., Karperien, M. \& Jonkheijm, P. A Microfluidic Device with Continuous Ligand Gradients in Supported Lipid Bilayers to Probe Effects of Ligand Surface Density and Solution Shear Stress on Pathogen Adhesion. Adv. Mater. Interfaces 3, 1600055 (2016).

32. Tong, W., Yao, X., Duan, S., Yu, B., Ding, X., Ding, X. \& Xu, F.-J. Gradient Functionalization of Various Quaternized Polyethylenimines on Microfluidic Chips for the Rapid Appraisal of Antibacterial Potencies. Langmuir 36, 354-361 (2020).

33. Huang, M. L., Cohen, M., Fisher, C. J., Schooley, R. T., Gagneux, P. \& Godula, K. Determination of receptor specificities for whole influenza viruses using multivalent glycan arrays. Chem. Commun. 51, 5326-5329 (2015).

34. Nicosia, C., Krabbenborg, S. O., Chen, P. \& Huskens, J. Shape-controlled fabrication of micron-scale surface chemical gradients via electrochemically activated copper(I) 'click' chemistry. J. Mater. Chem. B 1, 5417-5428 (2013).

35. Krabbenborg, S. O., Nicosia, C., Chen, P. \& Huskens, J. Reactivity mapping with electrochemical gradients for monitoring reactivity at surfaces in space and time. Nat. Commun. 4, 1667 (2013).

36. Van Weerd, J., Krabbenborg, S. O., Eijkel, J., Karperien, M., Huskens, J. \& Jonkheijm, P. On-Chip Electrophoresis in Supported Lipid Bilayer Membranes Achieved Using Low Potentials. J. Am. Chem. Soc. 136, 100-103 (2014).

37. Krabbenborg, S. O., van Weerd, J., Karperien, M., Jonkheijm, P. \& Huskens, J. Locked-in Biomimetic Surface Gradients that are Tunable in Size, Density and Functionalization. ChemPhysChem 15, 3460-3465 (2014).

38. Overeem, N. J., Hamming, P. H. \& Huskens, J. Time-Dependent Binding of Molecules and Nanoparticles at Receptor-Modified Supported Lipid Bilayer Gradients in a Microfluidic Device. ChemistrySelect 5, 9799-9805 (2020).

39. Jansen, A. J. G., Spaan, T., Low, H. Z., Di lorio, D., van den Brand, J., Tieke, M., Barendrecht, A., Rohn, K., van Amerongen, G., Stittelaar, K., Baumgärtner, W., Osterhaus, A., Kuiken, T., Boons, G.-J., Huskens, J., Boes, M., Maas, C. \& van der Vries, E. Influenza-induced thrombocytopenia is dependent on the subtype and sialoglycan receptor and increases with virus pathogenicity. Blood Adv. 4, 2967-2978 (2020).

40. Kausch, H. ., Fesko, D. . \& Tschoegl, N. . The random packing of circles in a plane. J. Colloid Interface Sci. 37, 603-611 (1971).

41. Dubacheva, G. V., Araya-Callis, C., Geert Volbeda, A., Fairhead, M., Codée, J., Howarth, M. \& Richter, R. P. Controlling Multivalent Binding through Surface Chemistry: Model Study on Streptavidin. J. Am. Chem. Soc. 139, 4157-4167 (2017).

42. Nagle, J. F. \& Tristram-Nagle, S. Structure of lipid bilayers. Biochim. Biophys. Acta-Rev. Biomembr. 1469, 159-195 (2000).

43. Di lorio, D. Designer Surfaces for the Quantification of Multivalent Biological Interactions. (2019). doi:10.3990/1.9789036548199

44. Nir, G., Chetrit, E., Vivante, A., Garini, Y. \& Berkovich, R. The role of near-wall drag effects in the dynamics of tethered DNA under shear flow. Soft Matter 14, 2219-2226 (2018).

45. Brown, D. L. Cardiovascular Plaque Rupture. (CRC Press, 2002). 


\section{Chapter 5}

46. Button, B. \& Boucher, R. C. Role of mechanical stress in regulating airway surface hydration and mucus clearance rates. Respir. Physiol. Neurobiol. 163, 189-201 (2008).

47. Button, B. M. \& Button, B. Structure and Function of the Mucus Clearance System of the Lung. Cold Spring Harb. Perspect. Med. 3, a009720 (2013).

48. Vanaki, S. M., Holmes, D., Jayathilake, P. G. \& Brown, R. Three-Dimensional Numerical Analysis of Periciliary Liquid Layer: Ciliary Abnormalities in Respiratory Diseases. Appl. Sci. 9, 4033 (2019).

49. Rimmelzwaan, G. F., Baars, M., Claas, E. C. J. \& Osterhaus, A. D. M. E. Comparison of RNA hybridization, hemagglutination assay, titration of infectious virus and immunofluorescence as methods for monitoring influenza virus replication in vitro. $J$. Virol. Methods 74, 57-66 (1998). 
Direct visualization of the superselective binding of influenza viruses 


\section{Chapter 6}

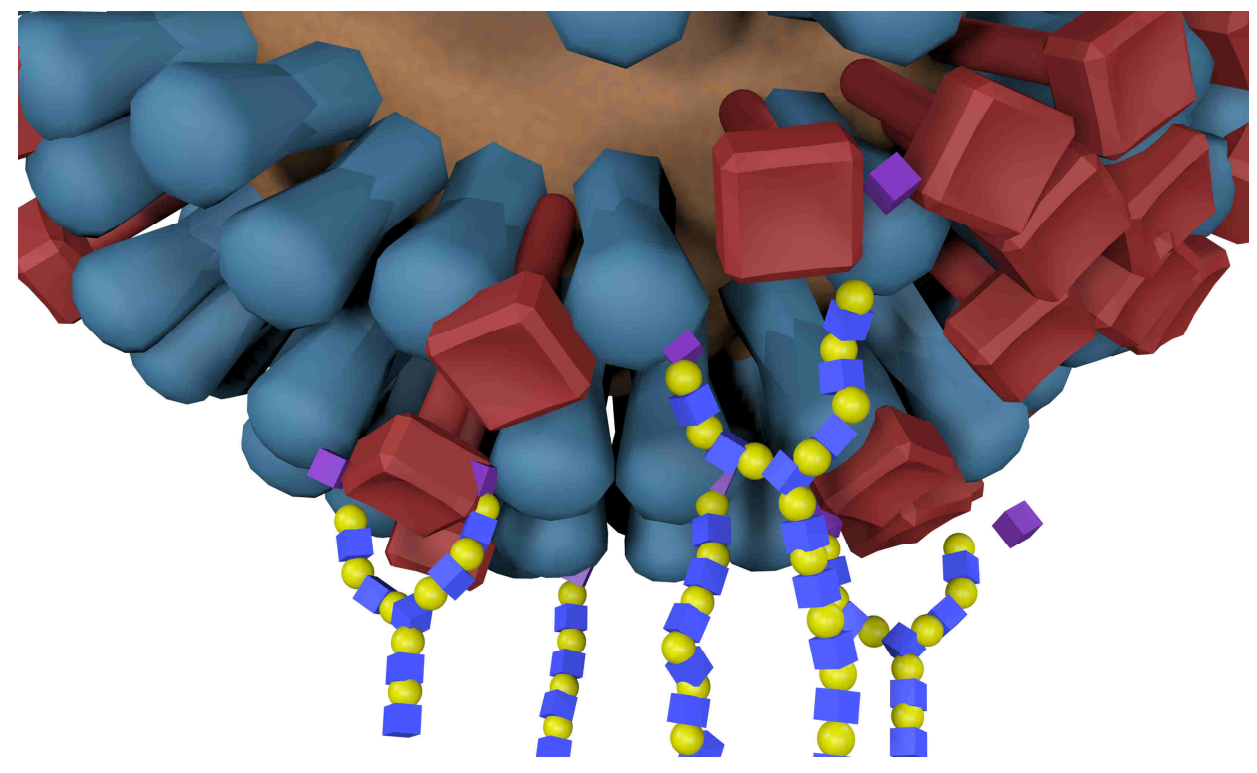

\section{Highlights}

- The binding of influenza viruses and density of receptors mutually influence each other

- The activity of neuraminidase (NA) during the binding is controlled by varying the concentration of NA inhibitor Zanamivir

- The receptor-cleaving activity increases the selectivity of influenza viruses towards initial receptor densities above the threshold 


\section{The role of neuraminidase in the superselective binding of the influenza virus}

Influenza A viruses (IAV)s infect host cells by binding to sialic acid-terminated glycans on the cell surface. The surface of IAV is decorated with two glycoproteins: hemagglutinin (HA), which binds sialoglycans, and neuraminidase (NA), which binds sialoglycans and cleaves the sialic acid from these glycans. This receptor-cleaving activity keeps the multivalent binding of $I A V$ reversible and provides a driving force that allows directional motility of the virus particles. In studies of virus binding, the receptor-cleaving activity of NA is usually suppressed using an inhibitor. This chapter studies the effect of active NA on the binding profiles of IAV as a function of receptor density. The activity of NA is controlled by varying the concentration of NA inhibitor. In the presence of inhibitor, the virus binding gradually increases over time. Without inhibitor, it levels off faster at high receptor densities, while at low receptor densities it first slowly increases and then decreases. This behavior enhances the selectivity towards areas with a higher receptor density progressively over time. A theoretical discussion of superselective virus binding shows that the contribution of NA to the binding leads to a higher avidity constant at the same receptor density, while the receptor density is reduced over time by the receptor-cleaving activity of NA. To study the role of NA at the level of individual viruses, we imaged the binding of viruses over time and observed motility only in a subpopulation of the adsorbed viruses of which very few showed longrange motility. The structure of the viruses was studied with cryo-TEM. Most virus particles are almost or completely spherical, but a subpopulation of elongated particles is present. This heterogeneity may play a role in the varied binding and motility of individual viruses. 


\subsection{Introduction}

Influenza A viruses (IAVs) cause respiratory disease in humans. Wild waterfowl are the reservoir for IAVs from which the viruses have spread and adapted to other species including humans, giving rise to several pandemics in the last century followed by seasonal epidemics. ${ }^{1}$ Changes in receptor specificity play an important role in this adaptation. ${ }^{2,3}$ IAV binds to sialic acid-terminated glycans through hemagglutinin (HA) glycoproteins on the surface of the virus. ${ }^{4}$ Whereas avian influenza HAs bind preferentially to sialic acid that is connected to a penultimate galactose by an $\alpha 2,3$-linkage, human influenza HAs favor the $\alpha 2,6$-linkage. ${ }^{5}$ This difference is attributed to differences in the glycocalyces of the host cells. ${ }^{6}$ Whereas avian intestines are rich in $\alpha 2,3$-linked sialic acid, $\alpha 2,6$ linked sialic acids prevail in the human upper airways. ${ }^{7}$

A second surface glycoprotein of IAV, the enzyme neuraminidase (NA), binds to the same glycan receptors as HA and cleaves the bond between sialic acid and galactose. ${ }^{8,9}$ This allows progeny viruses to leave an infected host cell and infect other cells. ${ }^{10}$ The cleavage of receptors also helps to prevent off-target binding and keeps the multivalent binding of IAV in a dynamic regime (Chapter 2). ${ }^{11}$ Human-adapted IAVs generally retain a higher NA activity towards the $\alpha 2,3-$ linkage, which develops specificity for $\alpha 2,6$-linked sialic acids only after prolonged circulation in the human population. ${ }^{12,13}$ This is attributed to the presence of high numbers of $\alpha 2,3$-linked sialic acids in the mucus that protects the human airways, where they facilitate the clearance of pathogens by acting as decoy receptors. ${ }^{14,15}$

By cleaving receptors, NA not only prevents entrapment of IAV on the cell surface and in the mucus, but also provides a driving force that allows directional motility of IAV (Chapter 7). The directional motility of IAV is enhanced by the patchy distribution of HA and NA on the surface of IAV, especially in filamentous viruses. ${ }^{16}$ This allows the viruses to efficiently traverse the mucus layer and probe a host cell surface for a suitable location to induce endocytosis. Guo et al. showed that the binding of relatively few virus particles could remove sialic acid from a large sensor area. ${ }^{17}$

The NA inhibitors Zanamivir and Oseltamivir have shown some success as antiviral drugs and are recommended by the WHO for treatment of zoonotic 
and severe cases of seasonal influenza. ${ }^{18,19}$ These inhibitors interfere with sialidase activity by binding to the active site of NA to prevent the binding of sialic acid. ${ }^{8}$ When cells are incubated with IAV in the presence of an NA inhibitor, virus particles accumulate on the surface of the cell with very few infecting it. ${ }^{20}$

When the receptor specificity of influenza HA is studied, receptor cleaving is usually suppressed using NA inhibitors, or isolated HA is used. ${ }^{4,21-24}$ In Chapter 4 we showed that the structure of the multivalent virus-surface interaction interface plays a key role in receptor specificity. The specificity of the whole virus may therefore differ from the specificity of HA alone. Because virussurface interactions depend strongly on receptor density (Chapters 4 and 5), the manipulation of receptor density by NA activity cannot be ignored in binding assays of IAV.

Here we study the effect of the receptor-cleaving activity of influenza NA on the binding profile and selectivity of IAV as a function of receptor density. First, we study the time-dependent binding of IAV on receptor density gradients and control the activity of NA by varying the concentration of NA inhibitor. We evaluate the binding below and above the threshold receptor density to determine how NA affects the selectivity of IAV towards high receptor densities. We discuss how the contribution of NA affects the theoretical description of superselective virus binding. Lastly, we study the mobility and structure of individual virus particles.

\subsection{Results and discussion}

\subsubsection{Time-dependent binding studies of IAV with and without NA inhibitor}

Receptor gradients, to visualize the superselective binding of IAV and determine the threshold receptor density, were made in a microfluidic device as described in Chapter 4 (Figure 6.1a). The sensing area in this multivalent affinity profiling chip (MAP chip) consists of three positions each with 12-16 square corrals of $100 \mu \mathrm{m}$. Using the method described in Chapter 3, we formed SLBs from vesicles of zwitterionic MPPC (1-myristoyl-2-palmitoyl-sn-glycero-3-phosphocholine) with $0.3 \%$ biotin-DOPE (1,2-dioleoyl-sn-glycero-3-phosphoethanolamine-N(cap biotinyl)), which has a negative charge. Electrophoretic gradients were formed by applying a potential of $2.0 \mathrm{~V}$ over the SLBs for $30 \mathrm{~min}$. The resulting 
biotin gradients were subsequently modified with fluorescently labelled

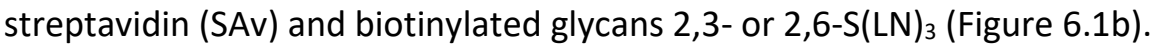

To study the influence of NA on the superselective binding of IAV, we performed time-dependent binding studies with and without the NA inhibitor Zanamivir (Figure 6.1c). We used fluorescently labelled IAV Puerto Rico/8/34 mt. Sinai strain (PR8), which was passed over the chips in a concentration of $10^{9} \mathrm{vp} / \mathrm{ml}$ at a flow rate of $1 \mu \mathrm{l} / \mathrm{min}$. Fluorescence micrographs were acquired at regular time intervals.

a

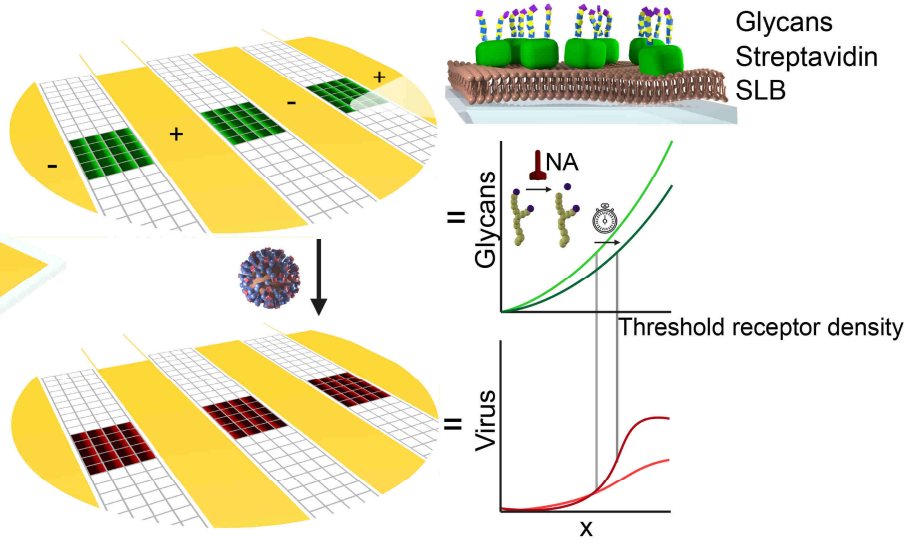

b
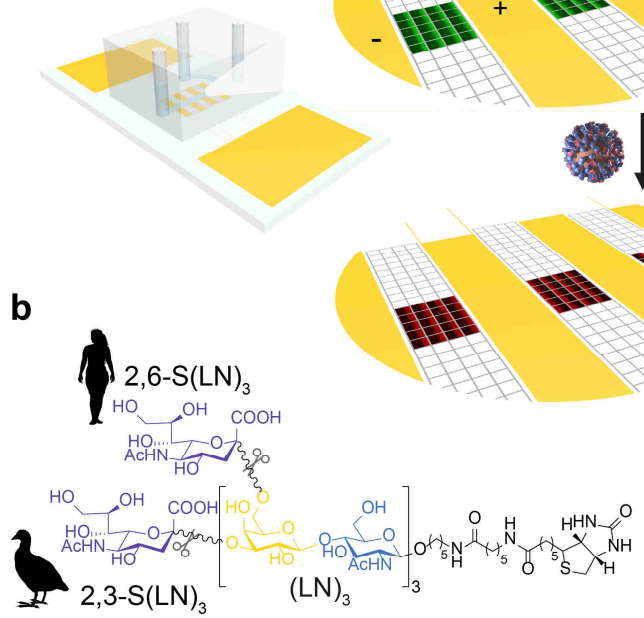

C

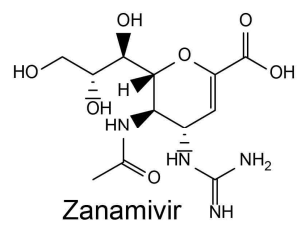

Figure 6.1| Method to study the influence of NA activity on superselective virus binding. a) Inside a microchannel on a chip, electrophoretic gradients are formed in SLBs. The gradients are functionalized with SAv and biotinylated glycans. Fluorescently labelled PR8 is passed over the gradients and its colocalization with SAv is imaged. Over time, the NA of PR8 cleaves off sialic acid residues, which leads to decreasing virus binding at low glycan density while viruses continue to bind where the receptor density is still high enough. As a result, the apparent threshold receptor density shifts together with the gradient. b) Structure of the biotinylated glycans 2,3-S(LN) (avian type) and

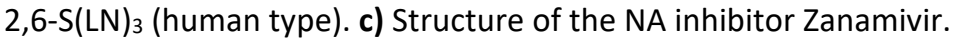


The development of virus binding profiles over time on surface gradients with $2,3$ and 2,6-S(LN $)_{3}$ with and without Zanamivir are shown in Figure 6.2a-d. Note that upon contact with active NA, we cannot estimate the absolute receptor density anymore, because the SAv fluorescence remains while the sialoglycans bound to it are being cleaved. As a result, the given receptor densities are initial densities, while the threshold receptor densities are apparent threshold receptor densities, as they are correlated to the initial receptor densities. The virus binding is then determined as function of the initial receptor density, which was that before addition of the virus. Comparison of fluorescence intensities (Figure 6.2a-d insets) shows that fewer viruses adsorbed without Zanamivir. Virus binding was lowest for $2,3-\mathrm{S}(\mathrm{LN})_{3}$, which is likely due to the higher activity of PR8 NA towards the 2,3-linkage. ${ }^{25}$ In the presence of Zanamivir, the virus coverage increases gradually over all receptor densities but faster at high receptor densities (Figure 6.2e-f). In the absence of Zanamivir, the virus coverage levels off more quickly at high receptor densities, while at low receptor densities it first rises and then decreases (Figure 6.2g-h). This suggests that the receptor-cleaving activity of NA leads to locally decreasing receptor densities that do no longer retain viruses, which occurs quicker at low initial receptor densities.

On $2,3-\mathrm{S}(\mathrm{LN})_{3}$ surface gradients, the virus coverage leveled off much quicker without Zanamivir than on 2,6-S(LN) $)_{3}$, which is consistent with the difference in NA activity (cf. Figure $6.2 \mathrm{~g}$ and $6.2 \mathrm{~h}$ ). The threshold receptor density with Zanamivir stabilized at a lower value than without (Figure 6.2i-j). The difference in threshold density with and without Zanamivir was similar for 2,3-S(LN) ${ }_{3}$ and $2,6-\mathrm{S}(\mathrm{LN})_{3}$. For $2,6-\mathrm{S}(\mathrm{LN})_{3}$ with Zanamivir, the threshold receptor density appeared to decrease after an initial peak, which may be related to the less regular increase in fluorescence intensity (Figure 6.2f). Without Zanamivir, the apparent threshold receptor density on $2,6-\mathrm{S}(\mathrm{LN})_{3}$ increased slowly over time. For $2,3-\mathrm{S}(\mathrm{LN})_{3}$ no significant change in threshold receptor density is visible over time after the initial stabilization, neither with nor without Zanamivir. 


\section{Chapter 6}

a

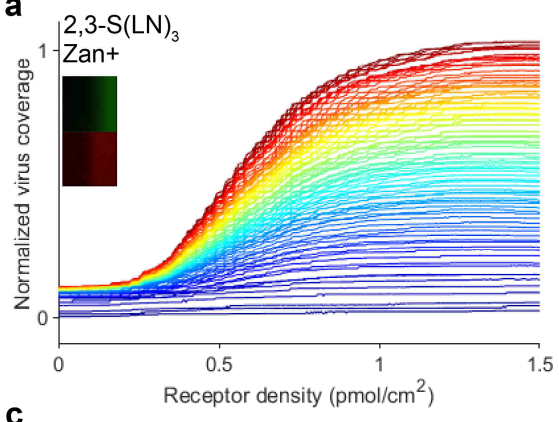

C

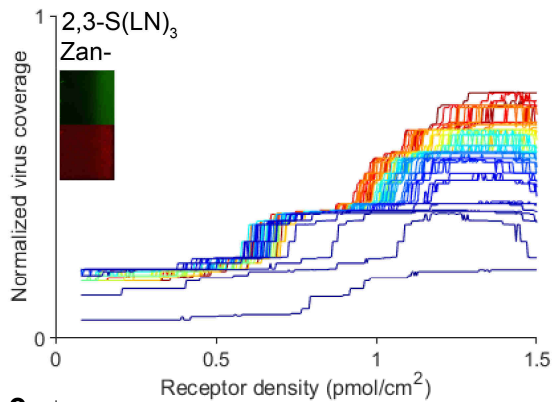

e
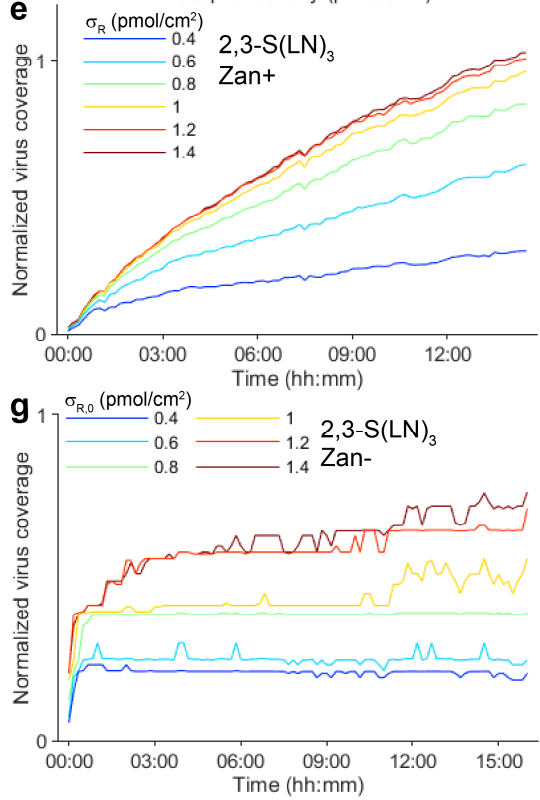

b

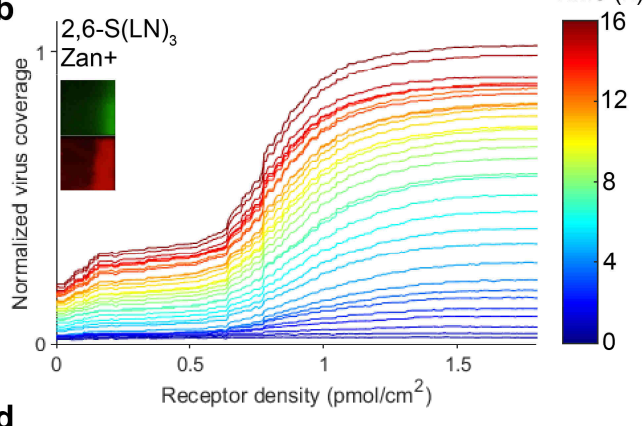

d

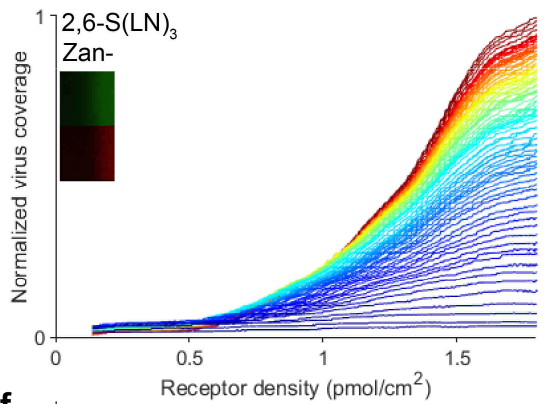

f

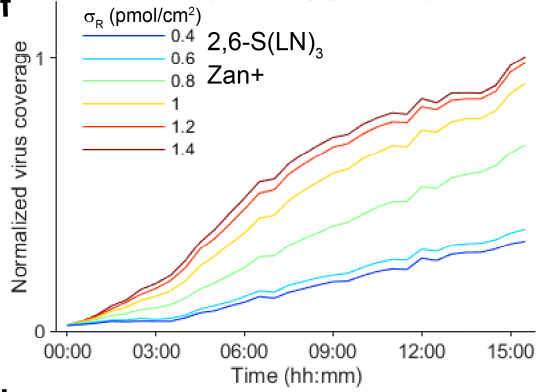

$\left.\mathbf{h}^{1}\right] \sigma_{\mathrm{R} 0}\left(\mathrm{pmol}^{2} \mathrm{~cm}^{2}\right)$

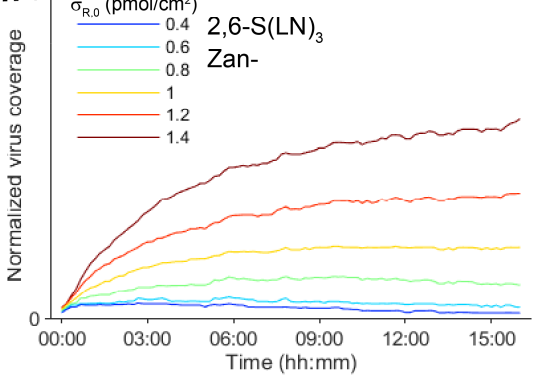



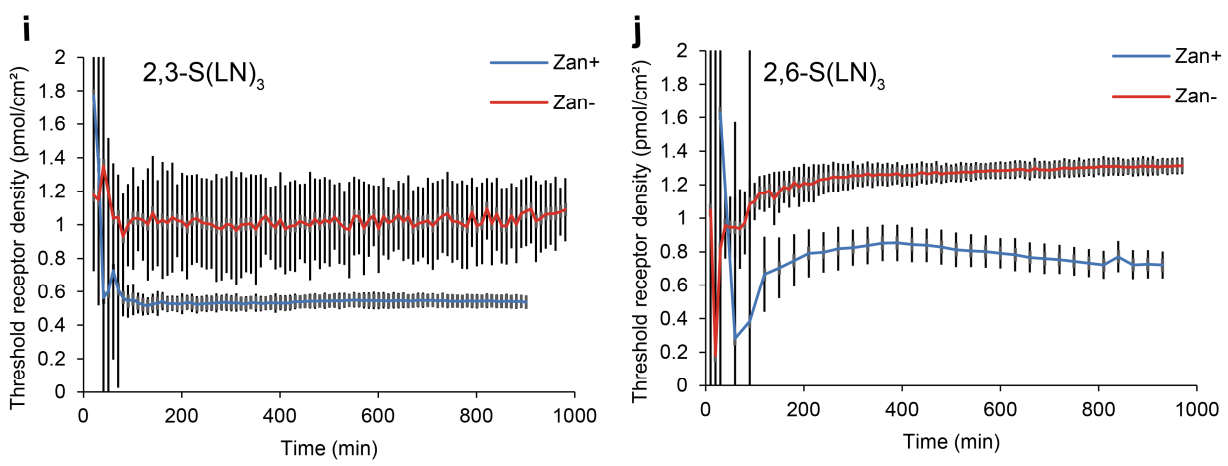

$\Delta$ Figure 6.2 | Time-dependent binding profiles of IAV as a function of receptor density in the presence and absence of NA inhibitor. a-d) Binding profiles of PR8 as function of initial receptor density on 2,3-S(LN) $3(\mathbf{a}, \mathbf{c})$ and 2,6-S(LN) $)_{3}(\mathbf{b}, \mathbf{d})$ with $200 \mu \mathrm{M}$ $(\mathbf{a}, \mathbf{b})$ and without (c, d) Zanamivir. The binding profiles are normalized between the minimum of the first frame and the maximum of the last frame. Each curve represents the median virus coverage in a rolling window of 50 for 1001 points along the receptor densities. The color scale represents the time point $(0-16 \mathrm{~h})$ at which the micrograph was acquired. Insets show micrographs of the SAv gradients (green) and final virus profiles (red). For visibility, the brightness of the green micrographs is enhanced in $b$, and of the red micrographs in c and d. e-h) Binding profiles of PR8 as function of time for a selection of initial receptor densities on 2,3-S(LN) $3(\mathbf{e}, \mathbf{g})$ and 2,6-S(LN) $)_{3}(\mathbf{f}, \mathbf{h})$ with $200 \mu \mathrm{M}(\mathbf{e}, \mathbf{f})$ and without (g, h) Zanamivir. i) Development of the (apparent) threshold receptor density over time on 2,3-S(LN) 3 with and without Zanamivir. j) Development of the (apparent) threshold receptor density over time on $2,6-\mathrm{S}(\mathrm{LN})_{3}$ with and without Zanamivir.

The receptor-cleaving activity of NA can be controlled by varying the concentration of NA inhibitor. ${ }^{26}$ We measured time-dependent binding profiles of PR8 on 2,3-S(LN) $)_{3}$ gradients for varying concentrations of Zanamivir (Figure 6.3). Qualitatively, the binding profiles of PR8 with $1 \mu \mathrm{M}$ Zanamivir (Figure 6.3ab) resemble the profiles with $200 \mu \mathrm{M}$ Zanamivir as the virus coverage increases gradually at all receptor densities. However, at $1 \mu \mathrm{M}$, the virus binding levels off faster and the difference between low and high receptor densities is more pronounced than with $200 \mu \mathrm{M}$ Zanamivir. 


\section{Chapter 6}

a

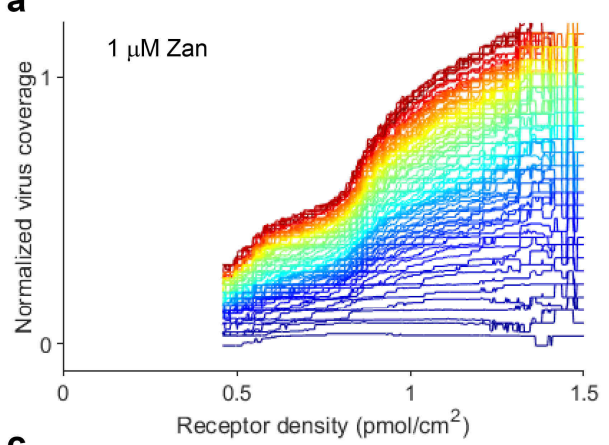

C
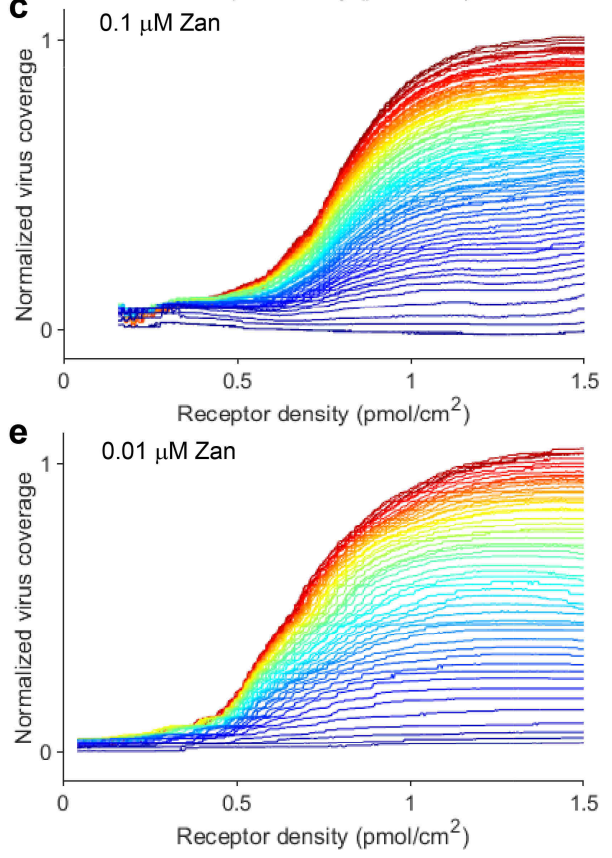

b $\quad \sigma_{R_{0}}\left(\mathrm{pmol} / \mathrm{cm}^{2}\right)$
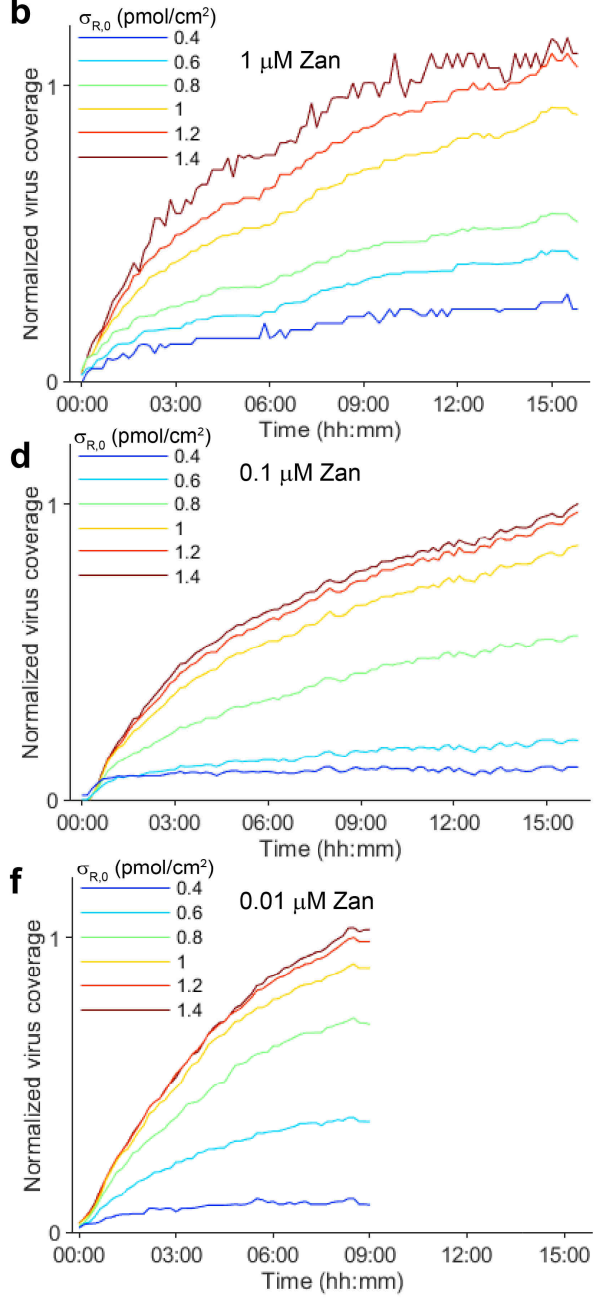

Figure 6.3| Binding profiles of PR8 on 2,3-S(LN) $)_{3}$ in the presence of varying concentrations of Zanamivir. a) Rolling window median binding profile of PR8 with 1 $\mu \mathrm{M}$ Zanamivir as function of initial receptor density. b) Binding profiles of PR8 as function of time for a selection of initial receptor densities with $1 \mu \mathrm{M}$ Zanamivir. c-d) Binding profiles with $0.1 \mu \mathrm{M}$ Zanamivir. e-f) Binding profiles with $0.01 \mu \mathrm{M}$ Zanamivir. 
The binding profiles with $0.1 \mu \mathrm{M}$ (Figure 6.3c-d) and $0.01 \mu \mathrm{M}$ Zanamivir (Figure 6.3e-f) resemble those without Zanamivir as the virus binding at low initial receptor densities first increases and then decreases. At high receptor densities, the virus binding does not level off as quickly as without Zanamivir on $2,3-\mathrm{S}(\mathrm{LN})_{3}$ but resembles the profile on $2,6-\mathrm{S}(\mathrm{LN})_{3}$. This suggests that the differences in binding profiles on 2,3-S(LN) $)_{3}$ and 2,6-S(LN) $)_{3}$ without Zanamivir are due to the difference in NA activity towards the different linkages.

We determined the threshold receptor densities of PR8 as described in Chapter 4 and 5 and compare them for 2,3-S(LN) $)_{3}$ and 2,6-S(LN) $)_{3}$ in the presence of different concentrations of Zanamivir (Figure 6.4a). The threshold receptor density without Zanamivir was higher than with $200 \mu \mathrm{M}$ Zanamivir $(p<0.001)$. The difference in threshold receptor density between $2,3-\mathrm{S}(\mathrm{LN})_{3}$ and $2,6-\mathrm{S}(\mathrm{LN})_{3}$ without Zanamivir was not significant $(p>0.05)$. For Zanamivir concentrations of $1 \mu \mathrm{M}, 0.1 \mu \mathrm{M}$, and $0.01 \mu \mathrm{M}$ Zanamivir, the threshold receptor density decreased progressively. This suggests a minimum may exist in the threshold receptor density as function of NA activity.

To understand how the different threshold receptor densities arise from the different time-dependent binding profiles, we compared the adsorption rates above and below the threshold receptor density. Because we did not measure the adsorption rate directly, we assume that the initial adsorption rate is the same for all concentrations of Zanamivir in the first $100 \mathrm{~min}$ and normalize $\theta$ accordingly:

$\theta_{\text {norm }}(t)=\theta(t) \frac{\Delta \theta_{\max }(100 \mathrm{~min})}{100 \mathrm{~min}} / \frac{\Delta \theta_{\max , 200 \mu \mathrm{M}(100 \mathrm{~min})}}{100 \mathrm{~min}}$

with $\theta(t)$ the virus coverage that was normalized such that $\theta_{\min }\left(t_{\min }\right)=0$ and $\theta_{\max }\left(t_{\max }\right)=1, \frac{\Delta \theta_{\max }(100 \mathrm{~min})}{100 \mathrm{~min}}$ the slope of maximum virus coverage at the highest receptor densities $\theta_{\max }$ in the first $100 \mathrm{~min}$, and $\frac{\Delta \theta_{\max , 200 \mu \mathrm{M}}(100 \mathrm{~min})}{100 \mathrm{~min}}$ the slope of $\theta_{\max }$ in the first $100 \mathrm{~min}$ at a Zanamivir concentration of $200 \mu \mathrm{M}$ (Figure 6.4b). This normalization allows us to compare the virus binding at different receptor densities for different concentrations of Zanamivir but may underestimate $\theta$ if NA contributes strongly to the initial binding rate. 


\section{Chapter 6}

a

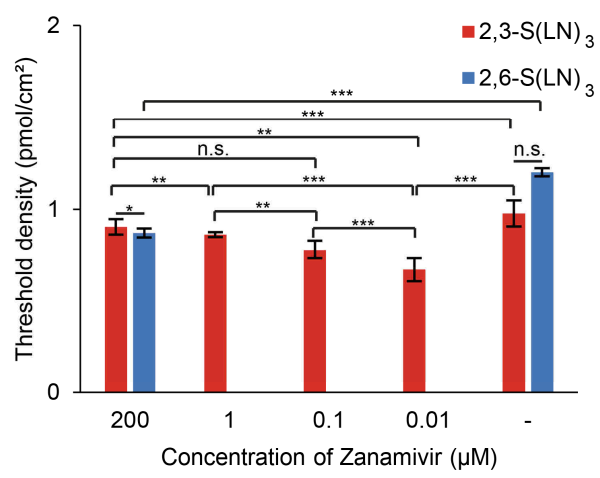

C $2,3-\mathrm{S}(\mathrm{LN})_{3} \sigma_{\mathrm{R}, 0}=0.5 \mathrm{pmol} / \mathrm{cm}^{2}$

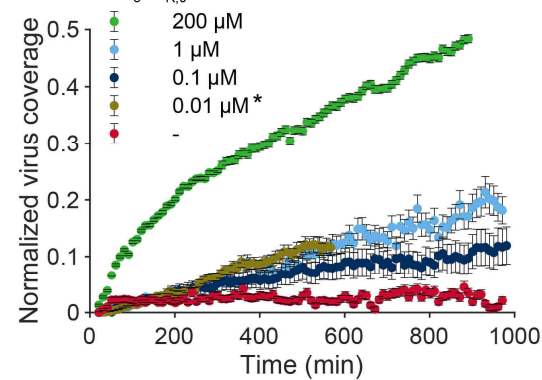

e

$2,6-\mathrm{S}(\mathrm{LN})_{3} \sigma_{\mathrm{R}, 0}=0.5 \mathrm{pmol} / \mathrm{cm}^{2}$

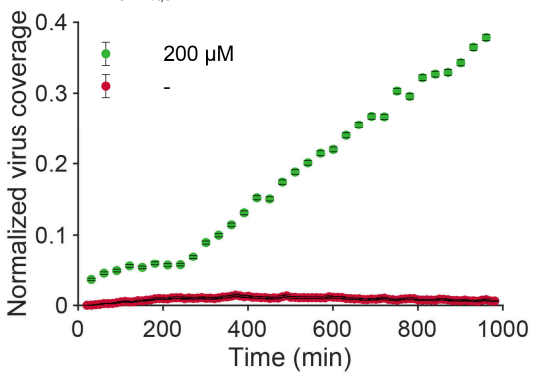

g

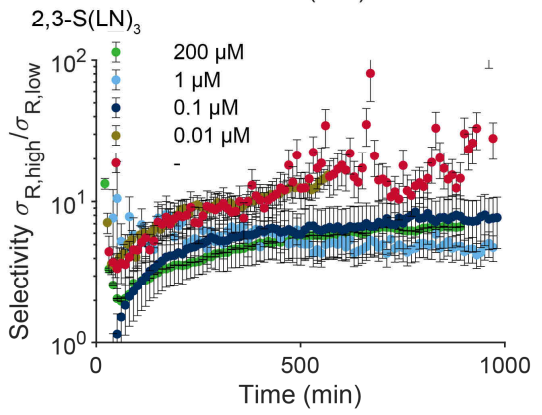

b $2,3-\mathrm{S}(\mathrm{LN})_{3}$

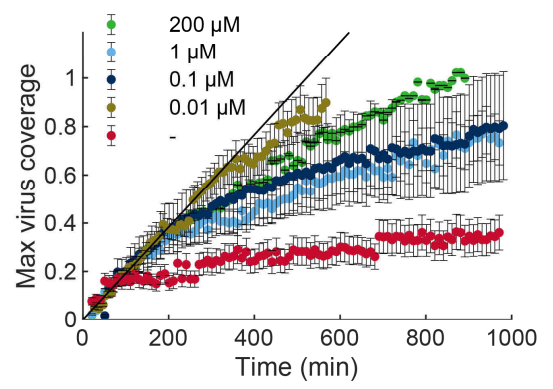

d $2,3-\mathrm{S}(\mathrm{LN})_{3} \sigma_{\mathrm{R}, 0}=1.2 \mathrm{pmol} / \mathrm{cm}^{2}$

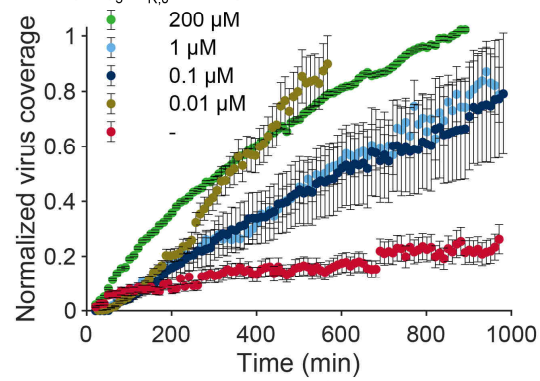

f $2,6-\mathrm{S}(\mathrm{LN})_{3} \sigma_{\mathrm{R}, 0}=1.5 \mathrm{pmol} / \mathrm{cm}^{2}$
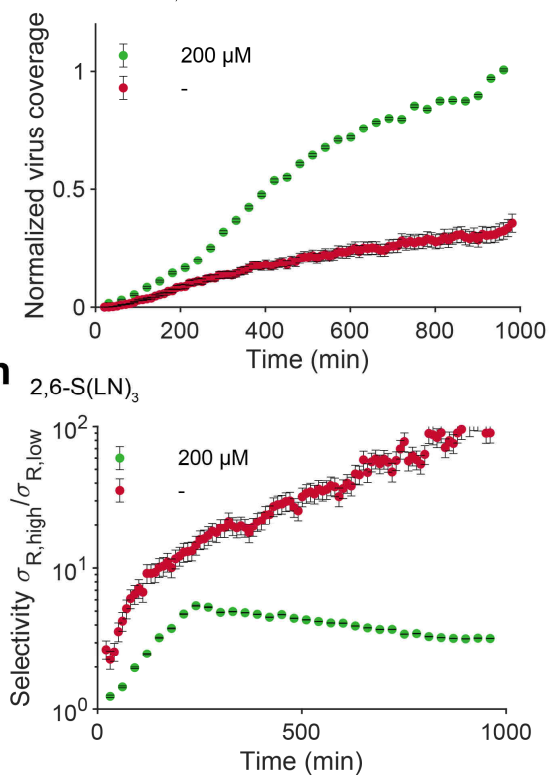
4Figure 6.4| Binding of PR8 vs time and receptor density for varying Zanamivir concentrations. a) Comparison of (apparent) threshold receptor densities for varying concentrations of Zanamivir. Error bars indicate the $95 \%$ confidence interval over different corrals. n.s.: not significant, $*$ : $p<0.05, * *: p<0.01, * * *$ : $p<0.001$. b) Maximum virus coverage over time in binding studies with varying concentration of Zanamivir (Figure 6.2a and c, and Figure 6.S1), normalized by initial binding rate to the maximum virus coverage with $200 \mu \mathrm{M}$ Zanamivir. c) Mean virus coverage at an initial $2,3-\mathrm{S}(\mathrm{LN})_{3}$ density of $0.5 \pm 0.01 \mathrm{pmol} / \mathrm{cm}^{2}$ over time. For $0.01 \mu \mathrm{M}$ Zanamivir, the mean virus coverage was evaluated at $0.4 \pm 0.01 \mathrm{pmol} / \mathrm{cm}^{2}$ because the threshold receptor density was lower. d) Mean virus coverage at an initial 2,3-S(LN) 3 density of $1.2 \pm 0.01$ $\mathrm{pmol} / \mathrm{cm}^{2}$ over time. e) Mean virus coverage at an initial 2,6-S(LN) 3 density of $0.5 \pm 0.01$ $\mathrm{pmol} / \mathrm{cm}^{2}$ over time. f) Mean virus coverage at an initial 2,6-S(LN) ${ }_{3}$ density of $1.5 \pm 0.01$ $\mathrm{pmol} / \mathrm{cm}^{2}$ over time. g) Development over time of the selectivity of PR8 for high 2,3$\mathrm{S}(\mathrm{LN})_{3}$ density as ratio of the mean virus coverage at $\sigma_{\mathrm{R}, 0}>1.3 \mathrm{pmol} / \mathrm{cm}^{2}$ over the mean virus coverage at $\sigma_{R, 0}<0.5 \mathrm{pmol} / \mathrm{cm}^{2}$. The $y$-scale is logarithmic. h) Development over time of the selectivity of PR8 for high $2,6-\mathrm{S}(\mathrm{LN})_{3}$ density as ratio of the mean virus coverage at $\sigma_{\mathrm{R}, 0}>1.5 \mathrm{pmol} / \mathrm{cm}^{2}$ over the mean virus coverage at $\sigma_{\mathrm{R}, 0}<0.5 \mathrm{pmol} / \mathrm{cm}^{2}$.

The slope of $\theta_{\max }$ levels off similarly for $0.01 \mu \mathrm{M}-200 \mu \mathrm{M}$ Zanamivir after the first $100 \mathrm{~min}$, but much more strongly without Zanamivir (Figure 6.4b). At receptor densities just below the threshold (Figure 6.4c), the virus coverage with a 0.01-1 $\mu \mathrm{M}$ Zanamivir increased much slower than with $200 \mu \mathrm{M}$ but does not level off. While the coverage without Zanamivir initially followed the coverage with 0.01-1 $\mu \mathrm{M}$ Zanamivir, it proceeded to decrease. Just above the threshold receptor density (Figure 6.4d), the virus coverage with $200 \mu \mathrm{M}$ Zanamivir followed the same profile as at $\theta_{\max }$, whereas the coverage with 0.01 $1 \mu \mathrm{M}$ Zanamivir increased more slowly but did not level off. The coverage without Zanamivir leveled off more quickly than at $\theta_{\max }$ so that it initially followed the coverage with 0.01-1 $\mu \mathrm{M}$ Zanamivir. For 2,6-S(LN) $)_{3}$, the slope of $\theta_{\mathrm{max}, 200 \mu \mathrm{M}}$ was evaluated over the entire study rather than the first $100 \mathrm{~min}$ because its initial shape was irregular. The binding profiles on 2,6-S(LN) $)_{3}$ with and without Zanamivir shows qualitatively the same trends as on $2,3-\mathrm{S}(\mathrm{LN})_{3}$ (Figure 6.4e-f). Above the threshold receptor density (Figure 6.4f), it levels off less than on 2,3-S(LN) $)_{3}$ but remains lower than the coverages for $0.01-1 \mu \mathrm{M}$ Zanamivir.

The relative differences in coverage at different receptor densities for different concentrations of Zanamivir suggest differences in selectivity towards high 
receptor densities. We evaluate the mean normalized virus binding above and below the threshold and calculate the ratio:

$$
s=\frac{\theta_{\mathrm{Zan}+}\left(\sigma_{\mathrm{R}, 0_{\mathrm{high}}}\right)}{\theta_{\mathrm{Zan}+}\left(\sigma_{\mathrm{R}, 0_{\mathrm{low}}}\right)}
$$

with $\sigma_{\mathrm{R}, 0_{\text {high }}}$ the initial receptor density above the threshold and $\sigma_{\mathrm{R}, 0_{\text {low }}}$ below (Figure 6.4g-h). This practical selectivity parameter $s$ is not to be confused with the superselectivity parameter $\alpha=\ln (\theta) / \ln \left(\sigma_{\mathrm{R}}\right)$ that is widely used to assess the slope of the binding profile as function of receptor density. ${ }^{27-33}$ Over time, as more virus bound, the selectivity $s$ stabilized in the presence of $200 \mu \mathrm{M}$ Zanamivir at values of 6.6 for $2,3-\mathrm{S}(\mathrm{LN})_{3}$ and 3.2 for $2,6-\mathrm{S}(\mathrm{LN})_{3}$. Without Zanamivir, the selectivity increased exponentially, while the virus binding at low densities decreased, to values for $s$ of around 30 for 2,3-S(LN) $)_{3}$ and 100 for 2,6$\mathrm{S}(\mathrm{LN})_{3}$. At $1 \mu \mathrm{M}$ and $0.1 \mu \mathrm{M}$ Zanamivir, the selectivity followed closely that at $200 \mu \mathrm{M}$, but at $0.01 \mu \mathrm{M}$ Zanamivir, the selectivity followed that without Zanamivir. This shows that the selectivity of PR8 towards higher receptor densities is strongly enhanced by the receptor-cleaving activity of NA.

The virus binding profiles at different concentrations of Zanamivir suggest that NA has opposing effects on the virus binding. The threshold receptor density of PR8 on 2,3-S(LN) $)_{3}$ appears to decrease with decreasing concentrations of Zanamivir but is higher in the absence of Zanamivir (Figure 6.4a). This suggests that the threshold receptor density is affected by two opposing contributions of NA to the binding. Possibly, active NA increases the avidity of the virus, but if the NA activity is higher, its receptor-cleaving role decreases the number of receptors and consequently increases the apparent threshold receptor density. The virus binding at high receptor densities is almost equally affected by Zanamivir concentrations of $0.01 \mu \mathrm{M}-200 \mu \mathrm{M}$, whereas the virus binding with $0.01 \mu \mathrm{M}-1 \mu \mathrm{M}$ at low receptor densities is closer to the virus binding in the absence of Zanamivir than with $200 \mu \mathrm{M}$ (Figure 6.4b-d). This suggests that the receptor-cleaving activity of NA in the presence of $0.01 \mu \mathrm{M}-1 \mu \mathrm{M}$ remains high enough to effectively inhibit virus binding at low receptor densities but not at high receptor densities, whereas in the absence of Zanamivir the receptorcleaving activity of NA inhibits virus binding both at low and high receptor densities, thereby increasing the threshold receptor density. Yet, the virus binding in the absence of Zanamivir is still more effectively inhibited at low 
receptor densities than at higher ones, so that the selectivity of PR8 for high receptor densities increases with decreasing Zanamivir concentration i.e. higher NA activity.

This enhancement of selectivity by the receptor-cleaving activity of NA was also hinted at by Müller et al. who showed that the unbinding rate $k_{\text {off }}$ of IAV X31 (H3N2) on an SLB depends on the number of virus-receptor interactions and decreases with increasing concentration of Zanamivir. ${ }^{26}$ They measured the diffusion coefficient $D$ of virus particles that were bound to glycolipids in a fluid $\mathrm{SLB}$, which scales with the number of virus-receptor interactions. At high concentrations of Zanamivir, $k_{\text {off }}$ decreases monotonically, but at low concentrations, $k_{\text {off }}$ exhibits a peak at $1 / D=5 \mathrm{~s} / \mu \mathrm{m}^{2}$. They found that the $\mathrm{IC}_{50}$ of Zanamivir at this peak was $2.4 \mu \mathrm{M}$, which is within the range we studied. They did not investigate how the average number of interactions was affected by Zanamivir. The existence of this peak in $k_{\text {off }}$ may explain why the virus binding is more strongly affected by Zanamivir below the threshold receptor density than above. This peak in $k_{\text {off }}$ suggests that the receptor-cleaving activity leads only to effective unbinding of the virus if the number of virus-receptor interactions is sufficiently high to have both HA and NA present in the contact area but low enough that the virus has mobility to allow NA to probe and cleave sufficient receptors in the contact area to lead to unbinding. The higher $k_{\text {off }}$ for a lower number of interactions matches the lower virus binding that was observed at lower receptor densities with low concentrations or absence of Zanamivir. Consequently, the enhancement in selectivity by the receptorcleaving activity of NA may be attributed to the non-monotonic increase in $k_{\text {off. }}$.

6.2.2 Towards a theoretical model of superselective virus binding with receptor cleaving

To understand the influence of receptor cleaving on the time-dependent binding of IAV, a model is needed that describes both the superselective binding and the influence of receptor cleaving by bound virus particles on the density of receptors. The binding profile of IAV as a function of receptor density, in the presence of NA inhibitor, is described with a theoretical binding model (see Chapter 4), based on the statistical thermodynamics of multivalent adsorption: ${ }^{26,30}$ 
$\theta=\frac{K_{\mathrm{av}}\left(\sigma_{\mathrm{R}}\right)[V]}{1+K_{\mathrm{av}}\left(\sigma_{\mathrm{R}}\right)[V]}$

where $\theta$ is the virus coverage, [ $V$ the virus concentration, and $K_{\mathrm{av}}\left(\sigma_{\mathrm{R}}\right)$ is the multivalent equilibrium binding constant that is a function of receptor density:

$K_{\mathrm{av}}\left(\sigma_{\mathrm{R}}\right)=N_{\mathrm{A}} V_{\mathrm{ex}}\left(1+\frac{K_{\mathrm{i}, \text { eff }}}{N_{\mathrm{A}} V_{\text {explore }}}\right)^{A_{\text {contact }} \min \left(\sigma_{\mathrm{R}}, \sigma_{\mathrm{HA}}\right)}$

where $N_{\mathrm{A}}$ is Avogadro's number, $V_{\text {ex }}$ the excluded volume of a virus particle, $K_{\text {i,eff }}$ the effective equilibrium constant of an individual HA-glycan interaction, $V_{\text {explore }}$ the volume accessible to a glycan, $A_{\text {contact }}$ the surface area that can be reached by $\mathrm{HA}$ when the virus is bound, and $\min \left(\sigma_{\mathrm{R}}, \sigma_{\mathrm{HA}}\right)$ the minimum of the density of receptors on the surface and receptor binding domains of HA on the virus, which is usually $\sigma_{\mathrm{R}}$ in this study. The product $A_{\text {contact }} \min \left(\sigma_{\mathrm{R}}, \sigma_{\mathrm{HA}}\right)$ is the number of possible simultaneous virus-receptor interactions a virus can form on average.

If NA is not inhibited, it can participate in the binding together with HA. Because Equation 6.4 is valid for a regime where ligands and receptors are short with limited configurational flexibility so that each may only bind to the nearest partner withing a given range (Chapter 4 ) and $\sigma_{\mathrm{R}}<\sigma_{\mathrm{HA}}$, the equation is only affected by the contribution of NA if the $K_{\mathrm{i} \text {,eff }}$ of NA is higher than the $K_{\mathrm{i} \text {,eff }}$ of $\mathrm{HA}$. Because HA and NA are not uniformly distributed over the surface of the virus, the $K_{\mathrm{i}, \text { eff }}$ will then become a weighted average of NA and HA.

A higher value of $K_{\mathrm{i}, \text { eff }}$ for NA will manifest in a lower threshold receptor density. Meanwhile, the receptor-cleaving activity of NA will decrease $\sigma_{\mathrm{R}}$ over time which leads to a higher apparent threshold receptor density. Figure 6.4a shows that the threshold receptor density decreases at low concentrations of Zanamivir, suggesting that indeed $K_{\mathrm{i} \text {,eff }}$ is higher for NA than for HA. In absence of Zanamivir, the higher $K_{\mathrm{i} \text {,eff }}$ of NA is masked by its receptor-cleaving activity. A quantitative assessment of the contribution of NA to virus binding may be possible by using a synthetic receptor analogue where the linkage between sialic acid and galactose is replaced by a thioglycosidic bond, which is not cleaved by NA. ${ }^{34,35}$ 
In addition to the effect of NA on the binding, its receptor-cleaving activity decreases $\sigma_{R}$ over time. The receptor-cleaving activity can be described with a general enzymatic rate equation:

$\frac{d[\mathrm{~S}]}{d t}=-k_{\mathrm{cat}}[\mathrm{NA} \cdot \mathrm{S}]$

where $S$ is the substrate $\mathrm{S}(\mathrm{LN})_{3}$ that is cleaved by NA, $k_{\text {cat }}$ is the enzymatic rate constant, and [NA.S] the concentration of NA-S(LN) $)_{3}$ complexes. The concentration of NA-S(LN) $)_{3}$ complexes at the surface is:

$[\mathrm{NA} \cdot \mathrm{S}]_{\text {surf }}=[V]_{\text {surf }} \bar{N}_{\mathrm{NA}}$

with [ $\eta_{\text {surf }}$ the density of viruses on the surface and $\bar{N}_{\mathrm{NA}}$ the average number of NA-S(LN) $)_{3}$ interactions per virus. The density of surface-bound viruses is:

$[V]_{\text {surf }}=\frac{\theta}{N_{\mathrm{A}} A_{\mathrm{ex}}}$

where $A_{\text {ex }}$ is the excluded area of a virus particle $\pi\left(\frac{d}{2}\right)^{2}$. When applied to NA$\mathrm{S}(\mathrm{LN})_{3}$ complexes at the virus-surface interface, Equation 6.5 therefore takes the form:

$\frac{d \sigma_{\mathrm{R}}}{d t}=-k_{\text {cat }} \frac{\theta}{N_{\mathrm{A}} A_{\mathrm{ex}}} \bar{N}_{\mathrm{NA}}$

Here $\bar{N}_{\mathrm{NA}}$ depends on the ensemble average from the partition function that was used in Chapter 4 to derive Equation 6.4, which depends on the individual equilibrium constants $K_{\mathrm{i}}$ of both NA and HA and their relative abundance in the contact area. Because $\bar{N}_{\mathrm{NA}}$ itself is a function of $\sigma_{\mathrm{R}}$, Equation 6.8 cannot be fitted without further understanding of the contribution of NA to the binding. Therefore, both a quantitative experimental assessment of the contribution of NA to the binding and a deeper theoretical effort would be needed to assess the effect of the receptor-cleaving activity of NA on $\sigma_{\mathrm{R}}$ and thereby on the superselective binding of IAV.

\subsubsection{Interactions and structure of single virus particles}

To better understand the virus-receptor interactions, we studied the binding of individual virus particles. We used a higher magnification with oil immersion and a more powerful Andor Zyle $4.2 \mathrm{sCMOS}$ camera that allowed us to image 
individual virus particles with sufficient contrast over multiple frames. This was not possible with the Olympus DR70 camera as the R18 labels were fully quenched after fewer than 10 frames. We prepared 2,3-S(LN) $)_{3}$ gradients on SLBs with $0.5 \%$ biotin-DOPE and passed PR8 over them at a flow rate of 5 $\mu \mathrm{l} / \mathrm{min}$. Fluorescence micrographs were acquired at $5 \mathrm{~s}$ intervals.

In Figure 6.5, the receptor gradient and two consecutive frames showing the binding of viruses are compared. The superselectivity of IAV is also visible at the single virus level as more viruses can be distinguished in areas of high receptor density, although the difference is less pronounced than in a fully developed virus binding profile at low magnification. Over time, the number of virus particles increases as more and more viruses adsorb. One subpopulation of the adsorbed viruses unbinds within $5 \mathrm{~s}$, whereas other viruses remain on the surface indefinitely. Of these adsorbed viruses, some are fully immobile or move less than one $\mu \mathrm{m}$, whereas a handful of viruses move over a distance of several $\mu \mathrm{m}$ between frames. This long-range motility is observed primarily in newly adsorbed viruses, which slow down later.

The motility of IAV was attributed by Vahey and Fletcher to the asymmetric organization of HA and NA on the surface of elongated viruses. ${ }^{16}$ In this study,
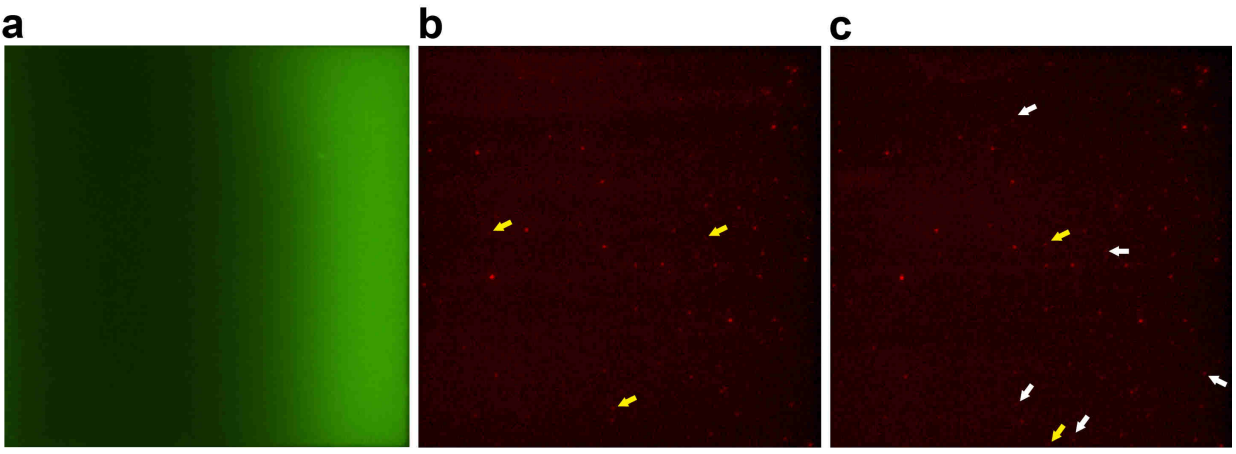

Figure 6.5 Adsorption of single PR8 particles. a) Fluorescence micrograph of a receptor density gradient in one $100 \mu \mathrm{m}$ corral. b) Fluorescence micrograph of PR8 viruses. Yellow arrows indicate viruses that are not present in the next micrograph. c) Fluorescence micrograph of PR8 viruses $5 \mathrm{~s}$ after the previous. Yellow arrows indicate transiently bound viruses that are not present in the previous and the next micrograph. White arrows indicate newly adsorbed viruses that are not present in the previous micrograph but remain in the next. 
however, we used egg-adapted PR8, which is reported to be highly spherical. ${ }^{36}$ In nanoparticle tracking analysis, the viruses in this study form predominantly one population with a median of $112 \mathrm{~nm}$, but several small subpopulations of approximately $60 \mathrm{~nm}, 150 \mathrm{~nm}, 200 \mathrm{~nm}, 260 \mathrm{~nm}$, and $300 \mathrm{~nm}$ can be seen (Figure 6.6a). Asymmetric organization of HA and NA on the surface of IAV was also reported in spherical particles of other egg-adapted viruses. ${ }^{37}$ To investigate the structure of the viruses that were used in this study, we used transmission electron microscopy of a cryogenically frozen PR8 solution (cryo-TEM). An overview of PR8 particles (Figure 6.6b) shows that most of the virus particles appear almost or completely spherical, but a significant minority of elongated virus particles is present as well. Some of the elongated virus particles have nicks that suggest that they may consist of two less elongated viruses that are superimposed in the image.
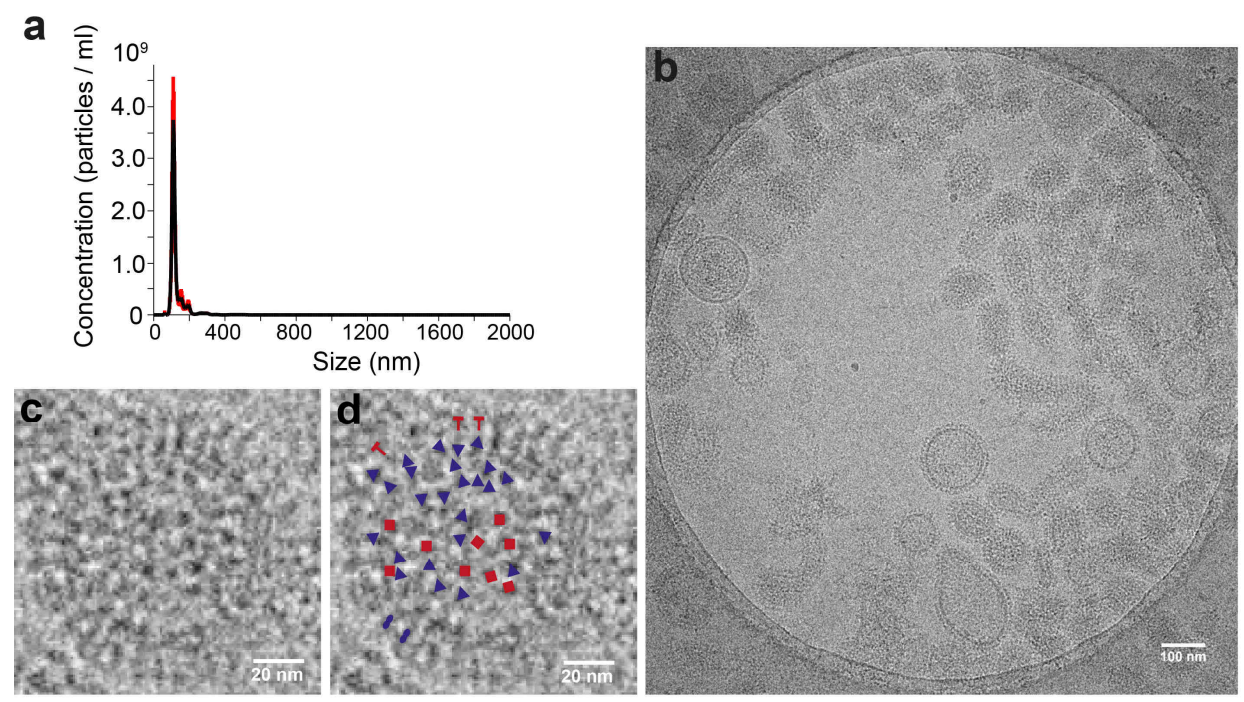

Figure 6.6| Cryo-TEM micrographs of PR8. a) Size distribution of PR8 virus particles as determined by nanoparticle tracking analysis. b) PR8 virus particles at 28,000x magnification. c) Detail of a single virus particle. d) Virus surface proteins HA (top view: blue triangles, side view: blue peanuts) and NA (top view: red squares, side view: red mushrooms) overlayed on the micrograph in c. 
One spherical virus where the surface proteins were visible was examined to resolve the distribution of HA and NA (Figure 6.6c). HA was identified from the top by its triangular shape, whereas NA has a square shape, and from the side by its bilobed "peanut" shape, whereas NA has a smaller head and longer stem, giving it a "mushroom" shape. Where HA and NA could be resolved, they are overlayed on the micrograph in Figure 6.6d. The distribution of HA and NA resembles that in other IAVs, with areas of only HA, smaller areas of only NA, and some isolated NA spikes surrounded by HA. ${ }^{37}$ This asymmetry in the distribution of HA and NA may be sufficient to allow motility through the mechanism proposed by Vahey and Fletcher, but likely with a weaker directionality. ${ }^{16}$

The presence of some elongated virus particles in a mostly spherical virus population leaves open the possibility that long-range motility is exclusive to elongated virus particles. The fact that elongated virus particles exist in eggadapted IAV strains despite not being selected for may be a feature of the lowfidelity assembly of IAV. ${ }^{38}$ The tendency of IAV to form pleiomorphic virus particles allows the virus to adapt to changes in environment, complementary to genetic adaptation. The differences in motility of individual virus particles is likely an effect of their morphological differences.

\subsection{Conclusions}

The time-dependent binding of PR8 on surface gradients of 2,3-S(LN) $)_{3}$ and 2,6$\mathrm{S}(\mathrm{LN})_{3}$ as a function of receptor density was studied in the absence and presence of varying concentrations of Zanamivir. We observed lower amounts of bound virus in the absence of Zanamivir, accompanied by an increase of the apparent threshold receptor density. However, at low concentrations of Zanamivir, the threshold receptor density decreased relative to higher concentrations of Zanamivir. This suggests that the inhibition of receptor cleaving is maintained at lower concentrations of Zanamivir compared to the inhibition of receptor binding by NA. The receptor-cleaving activity of NA diminishes the virus binding more strongly at low receptor densities, which leads to an enhanced selectivity of IAV towards higher receptor densities that strengthens over time. The contribution of NA to the binding leads to a higher avidity constant at the same receptor density, but the receptor density is reduced over time by the receptorcleaving activity of NA. The rate at which NA cleaves receptors depends on the 
number of bound viruses, the receptor density, and the individual equilibrium constants of complexation with NA and HA. At the level of individual viruses, the receptor-cleaving activity of NA allows motility over the surface, but this is only observed in a subpopulation of the adsorbed viruses of which very few show long-range motility.

\subsection{Acknowledgements}

Erik Hamming is thanked for his role in the image analysis and the fitting of the theoretical models to experimental data. Nicholas Tito is thanked for his help with the theoretical models. Malte Tieke and Erhard van der Vries are thanked for the production and characterization of the viruses. Zeshi Li, Gaël Vos and Geert-Jan Boons are thanked for the synthesis of the glycans. Tren Fiers and Stephan Block are thanked for their role in the virus tracking experiments. Kai Ludwig is thanked for the (cryo-)TEM imaging. Wouter Vijselaar and Edo de Kruiff are thanked for the cleanroom fabrication of the gradient chips.

\subsection{Experimental}

\subsubsection{Materials}

Biotinylated glycans 2,6-S(LN) $)_{3}$ and 2,3-S(LN) $)_{3}$ were provided by Zeshi Li, Gaël Vos and Geert-Jan Boons, and synthesized as described in Chapter 4. All other starting materials and chemicals were purchased from Sigma-Aldrich, Fluka, Serva, Becton Dickinson, Avanti Polar Lipids, Acros organics, Microchem, Biotium, Thermo Fisher, Lonza, Schott and Fujifilm, and they were used as received, unless otherwise stated.

\subsubsection{Virus stock preparation}

Influenza A/Puerto Rico/8/34 virus (Mt. Sinai strain) stocks were prepared by propagating the virus in 10-days-old embryonated chicken eggs (GD Animal health, Deventer, The Netherlands) at $33^{\circ} \mathrm{C}$. Allantoic fluids were harvested after 48-72 $\mathrm{h}$ and cleared from debris by centrifugation at 3,000 rpm for $10 \mathrm{~min}$ at $4{ }^{\circ} \mathrm{C}$. Subsequently, the viruses were pelleted by centrifugation at 7,000 rpm for $18 \mathrm{~h}$ at $4{ }^{\circ} \mathrm{C}$ and resuspended in PBS (pH 7.4) (Lonza). Viruses were then purified further by loading of the virus sample on a discontinuous sucrose gradient $(10-50 \% \mathrm{w} / \mathrm{v})$ and centrifugation for $45 \mathrm{~min}$ at 25,000 rpm using a SW41 swing-out rotor. The virus-containing sucrose layer was finally harvested 
and dialyzed (Slide-A-Lyzer, Thermo Scientific) for $48 \mathrm{~h}$ at $4{ }^{\circ} \mathrm{C}$ to remove the remaining sucrose. Finally, the virus particle count and size distribution were determined using a NS300 nanoparticle tracking analyzer (Malvern, Nanosight), and diluted to a final stock concentration of $1 \times 10^{11}$ particles $/ \mathrm{ml}$, UV-inactivated $\left(50 \mathrm{~mJ} / \mathrm{cm}^{2}\right.$, at a wavelength of $\left.365 \mathrm{~nm}\right)$ and aliquots were frozen at $-80^{\circ} \mathrm{C}$. Inactivation was confirmed by growing of these viruses on Madin-Darby canine kidney (MDCK) cells. The receptor binding capacity of the viruses was confirmed with a hemagglutination assay that was performed as described elsewhere ${ }^{39}$ to obtain a titer of 2048.

\subsubsection{Virus labelling}

Influenza viruses were fluorescently labelled using the lipophilic dye octadecyl rhodamine B (R18; Thermo Scientific), which binds the virus membrane with the fluorophore at the aqueous interface. R18 dye was mixed with inactivated virus stocks to a final concentration of $80 \mathrm{nM}$ and incubated in the dark for $2 \mathrm{~h}$ on ice. Free dye was subsequently separated from the R18-labelled viruses by adding $25 \mu \mathrm{l}$ Capto core 700 virus purification beads (GE Healthcare) per $1 \mathrm{ml}$ of virus and incubating by rotating the mixture for $30 \mathrm{~min}$ at $4^{\circ} \mathrm{C}$. Finally, the beads were cleared from the virus-containing supernatant by pelleting of the beads by centrifugation at $1,200 \mathrm{rpm}$ for $10 \mathrm{~min}$ in a table centrifuge at $4^{\circ} \mathrm{C}$.

\subsubsection{Chip fabrication}

Flow cells were fabricated according to the procedure described earlier in Chapter 3. A bilayer lift-off recipe was used for fabricating Au electrodes on Mempax glass wafers of $0.2 \mathrm{~mm}$ and $0.5 \mathrm{~mm}$ thickness (Schott). First, LOR 5A (MicroChem) was spin-coated, after which normal lithography was performed on top with Olin OiR 907-17 photoresist (FujiFilm) to create a bilayer resist stack. Electrode patterns were made by exposing the photoresist through a patterned photomask and developing in Olin OPD 4262 (FujiFilm). The develop step washed away the exposed photoresist, and etching through the LOR 5A layer created an undercut. Then, $5 \mathrm{~nm} \mathrm{Ti}$ and $95 \mathrm{~nm}$ Au were deposited via e-beam evaporation (BAK 600, Balzers). The bilayer resist was then removed by sonication in acetone $(20 \mathrm{~min})$ and isopropanol $(10 \mathrm{~min})$ followed by $5 \mathrm{~min}$ immersion in OPD 4262, serving as a sacrificial layer to leave patterned $\mathrm{Au}$ electrodes on Mempax glass. To fabricate the $\mathrm{Cr}$ corrals (10 nm thick) in 
between the Au electrodes, the same procedure was performed a second time, but in this case following alignment with respect to the Au electrodes.

\subsubsection{PDMS flow channel}

Silicon flow channel masters were produced by standard photolithography steps and deep reactive ion etching. The polydimethylsiloxane (PDMS) flow channels were prepared from a degassed mixture of 10:1 Sylgard 184 elastomer and curing agent (Dow Corning Corp), which was cast onto the silicon master and cured at $60{ }^{\circ} \mathrm{C}$ overnight. The flow channels were cut to size and inlets and outlets were punched using a $1 \mathrm{~mm} \varnothing$ punch (Harris Uni-core, Sigma-Aldrich). After bonding to the chip, a flow channel of $6,000 \times 500 \times 50 \mu \mathrm{m}$ was prepared with a second channel entering from the side.

\subsubsection{PDMS bonding}

Chips were rinsed and sonicated extensively with acetone, ethanol and MilliQ water, and dried prior to UV-ozone exposure (UV/Ozone Procleaner plus, Bioforce Nanosciences) for at least $20 \mathrm{~min}$. After UV exposure, the chips were rinsed with ethanol and water, and dried under a stream of nitrogen. Both cutout PDMS flow channels and cleaned chips were treated with oxygen plasma for $30 \mathrm{~s}$ at $40 \mathrm{~W}$ (Plasma prep II, SPI supplies) after which they were bonded immediately. The chips were placed on a hot plate for $10 \mathrm{~min}$ at $70{ }^{\circ} \mathrm{C}$ to increase the binding strength. Tygon Microbore tubing S-54-HL (VWR, $0.25 \mathrm{~mm}$ inner $\varnothing$ and $0.75 \mathrm{~mm}$ outer $\varnothing$ ) of $80 \mathrm{~cm}$ for the inlets and $40 \mathrm{~cm}$ for the outlets was inserted into the PDMS. The assembled flow cell was placed in an oven at $60{ }^{\circ} \mathrm{C}$ for $1 \mathrm{~h}$. Leak-free operation was shown for flow rates up to $200 \mu \mathrm{L} / \mathrm{min}$.

\subsubsection{Lipid vesicle preparation}

MPPC (1-myristoyl-2-palmitoyl-sn-glycero-3-phosphocholine, Avanti) was stored as a $10 \mathrm{mg} / \mathrm{ml}$ stock solution in chloroform at $-20^{\circ} \mathrm{C}$. Biotin-cap-DOPE (1,2-dioleoyl-sn-glycero-3-phosphoethanolamine- $\mathrm{N}$-(cap biotinyl), Avanti) was stored as $0.2 \mathrm{mg} / \mathrm{ml}$ solution. Desired molar ratios were mixed in a glass vial, dried under a flow of nitrogen and kept under vacuum for at least $1 \mathrm{~h}$. The resulting film was resuspended by vortexing in MilliQ water at $50{ }^{\circ} \mathrm{C}$ to form multilamellar vesicles (MLVs) at $1 \mathrm{mg} / \mathrm{ml}$. The MLV solution was extruded 11 times through a $100 \mathrm{~nm}$ polycarbonate membrane (Avanti) at $50{ }^{\circ} \mathrm{C}$. The 
resulting large unilamellar vesicles (LUVs) were kept at room temperature and used within one week.

\subsubsection{SLB formation and functionalization}

Before SLB formation, the flow cells were washed with 2\% SDS (Sigma-Aldrich), then rinsed with MilliQ, and washed overnight with $2 \%$ Hellmanex $^{\mathrm{TM}}$ to activate the glass surface. Flow cells were mounted onto a heating plate using Scotch ${ }^{\text {TM }}$ tape and rinsed with MilliQ and PBS, while heating to $50^{\circ} \mathrm{C}$. Shortly before LUV incubation, the LUV solution was diluted to $0.5 \mathrm{mg} / \mathrm{ml}$ with PBS. The diluted LUV solution was passed through the flow cells through the secondary inlet for 30 $\min (10 \mu \mathrm{L} / \mathrm{min}$; primary inlet $1 \mu \mathrm{L} / \mathrm{min}$ PBS) to allow adsorption and rupture of the vesicles on the chips. The chips were then washed with MilliQ $(100 \mu \mathrm{L} / \mathrm{min}$ secondary inlet, $10 \mu \mathrm{L} / \mathrm{min}$ primary inlet), after which freshly prepared $0.5 \mathrm{mM}$ hydroxymethylferrocene ( $\mathrm{FcMeOH}, \mathrm{Acros})$ was passed through the device (50 $\mu \mathrm{L} / \mathrm{min}$, both inlets). A potential difference of $2.0 \mathrm{~V}$ was applied over the device for $30 \mathrm{~min}$ to induce electrophoresis in the SLBs. Subsequently, the chips were cooled rapidly on a heat exchanger to fix the surface gradient. The flow cells were then rinsed with MilliQ. Bovine serum albumin (Sigma) $50 \mathrm{mg} / \mathrm{ml}(0.5 \mathrm{ml}$, $10 \mu \mathrm{L} / \mathrm{min}$ ) was passed through the flow cells to form an antifouling layer on the tubing. The flow cells were rinsed with PBS. SAv with Alexa Fluor 488 label (Thermo Fischer) $20 \mu \mathrm{g} / \mathrm{ml}$ was passed through the secondary inlet $(10 \mu \mathrm{L} / \mathrm{min}$, primary inlet $1 \mu \mathrm{L} / \mathrm{min})$. The flow cells were rinsed with PBS. The SAv-modified SLBs were inspected with fluorescence microscopy on an Olympus inverted IX71 epi-fluorescence research microscope with X-cite 120PC mercury vapour lamp as light source and a digital Olympus DR70 camera for image acquisition. For Alexa Fluor 488, blue excitation ( $460 \leq \lambda_{\text {ex }} \leq 490 \mathrm{~nm}$ ) and green emission ( $505 \leq$ $\lambda_{\mathrm{em}} \leq 545 \mathrm{~nm}$ ) was filtered using the U-MWG2 Olympus filter cube. Glycan 2,6$\mathrm{S}(\mathrm{LN})_{3}$-biotin $500 \mathrm{nM}$ solutions were passed through the primary inlet (10 $\mu \mathrm{L} / \mathrm{min}$, secondary inlet $1 \mu \mathrm{L} / \mathrm{min}$ ), after which the flow cells were rinsed with PBS. During all steps, care was taken to ensure that no air bubbles entered the flow cell.

\subsubsection{Binding studies}

Solutions of IAV Puerto Rico/8/1934 mt. Sinai with R18 label were prepared at a concentration of $10^{9} \mathrm{vp} / \mathrm{ml}$ in PBS with 0-200 $\mu \mathrm{M}$ Zanamivir (GlaxoSmithKline). The virus solution was passed through the primary inlet of the flow cells at a 
flow rate of $10 \mu \mathrm{l} / \mathrm{min}$ for 2-3 min, after which the flow rate was reduced to 1 $\mu \mathrm{l} / \mathrm{min}$, with the secondary inlet PBS at $10 \%$ of the flow rate through the primary inlet. Fluorescence micrographs of the bound virus were acquired in the same position at regular intervals. After the $16 \mathrm{~h}$, the other positions in the microchannel were imaged as well. For R18, green excitation ( $510 \leq \lambda_{\text {ex }} \leq 550$ $\mathrm{nm})$ and red emission $\left(\lambda_{\mathrm{em}}>590 \mathrm{~nm}\right)$ was filtered.

\subsubsection{Image analysis}

8-bit RGB fluorescence micrographs were aligned and cropped into corrals using a custom written MATLAB program. The fluorescence intensities of receptor and virus labels in matching pixels were listed. The receptor density in each pixel was calculated by $\rho_{R, i}=\bar{\rho}_{\mathrm{R}} \cdot \frac{I_{i}-\bar{I}_{b g}}{\bar{I}-\bar{I}_{b g}}$, with $\bar{\rho}_{\mathrm{R}}$ the average receptor density, which is $2.77 x$ the biotin\% in the SLB, based on a $60 \AA^{2}$ lipid footprint, ${ }^{40,41} I_{\mathrm{i}}$ the local fluorescence intensity, $\bar{I}$ the average fluorescence intensity and $\bar{I}_{b g}$ the average fluorescence intensity of the background. The virus density was normalized between 0 and 1 by finding the maxima of $2 \mathrm{D}$ kernel estimations of the data points with the 50 lowest and $10 \%$ highest receptor densities. For timedependent binding studies, the minimum virus coverage is determined in the first frame and the maximum coverage in the last frame, and all binding profiles in a series are given the same normalization.

The median virus coverages in time-dependent studies are evaluated in a rolling window of 50 for 1001 points along the receptor densities axis. Threshold receptor densities were determined from binding profiles obtained by fitting Equation 6.4 with $K_{\mathrm{i}} / N_{\mathrm{A}} V_{\text {explore }}$ as fitting parameter, using the built-in fit function.

\subsubsection{Single virus tracking}

Flow cells with $0.2 \mathrm{~mm}$ glass were used to allow higher magnifications. Gradients were formed from SLBs with $0.5 \%$ biotin-DOPE and were functionalized with $S A v$ and $2,3-S(L N)_{3}$. Virus solution was passed through the microchannel at a flow rate of $5 \mu \mathrm{l} / \mathrm{min}$. Fluorescence micrographs were acquired on a Nikon Ti-E fluorescence microscope with perfect focus system and Andor Zyle $4.2 \mathrm{sCMOS}$ camera at 40x magnification with oil immersion. Blue excitation $\left(473 \leq \lambda_{\mathrm{ex}} \leq 491 \mathrm{~nm}\right)$ and green emission $\left(506 \leq \lambda_{\mathrm{em}} \leq 548 \mathrm{~nm}\right)$ were filtered to image the SAv gradient, and green excitation ( $542 \leq \lambda_{\text {ex }} \leq 582 \mathrm{~nm}$ ) 
and red emission $602 \leq \lambda_{\mathrm{em}} \leq 678 \mathrm{~nm}$ ) were filtered to image the viruses. An Andor Zyla VSC-07418 camera was used to acquire images in 16-bit color depth. Virus binding was studied by acquiring fluorescence micrographs at $5 \mathrm{~s}$ intervals.

\subsubsection{Cryo-TEM}

Viruses were concentrated to $\sim 10^{13} \mathrm{vp} / \mathrm{ml}$ in a $100 \mathrm{kDa}$ molecular weight cutoff Amicon Ultra $0.5 \mathrm{ml}$ spin filter. Cryo-TEM samples were prepared by placing 5 $\mu \mathrm{I}$ PR8 solution on a copper grid QUANTIFOIL holey carbon film type R 1.2/1.3, followed by blotting for $5 \mathrm{~s}$ and vitrification in liquid ethane using a Vitrobot Mark IV (FEI) automated blotting and freezing system. Cryo-TEM micrographs were acquired in a Talos Arctica (FEl) $200 \mathrm{kV}$ transmission electron microscope with field emission cathode, auto loader, Volta phase plate, and Falcon 3 Direct Electron Detector.

\subsection{References}

1. Krammer, F., Smith, G. J. D., Fouchier, R. A. M., Peiris, M., Kedzierska, K., Doherty, P. C., Palese, P., Shaw, M. L., Treanor, J., Webster, R. G. \& García-Sastre, A. Influenza. Nat. Rev. Dis. Prim. 4, 3 (2018).

2. Imai, M., Watanabe, T., Hatta, M., Das, S. C., Ozawa, M., Shinya, K., Zhong, G., Hanson, A., Katsura, H., Watanabe, S., Li, C., Kawakami, E., Yamada, S., Kiso, M., Suzuki, Y., Maher, E. a., Neumann, G. \& Kawaoka, Y. Experimental adaptation of an influenza H5 HA confers respiratory droplet transmission to a reassortant $\mathrm{H} 5 \mathrm{HA} / \mathrm{H} 1 \mathrm{~N} 1$ virus in ferrets. Nature 486, 420-428 (2012).

3. Song, H., Qi, J., Xiao, H., Bi, Y., Zhang, W., Xu, Y., Wang, F., Shi, Y. \& Gao, G. F. Avian-toHuman Receptor-Binding Adaptation by Influenza A Virus Hemagglutinin H4. Cell Rep. 20, 1201-1214 (2017).

4. Xiong, X., Coombs, P. J., Martin, S. R., Liu, J., Xiao, H., McCauley, J. W., Locher, K., Walker, P. A., Collins, P. J., Kawaoka, Y., Skehel, J. J. \& Gamblin, S. J. Receptor binding by a ferrettransmissible H5 avian influenza virus. Nature 497, 392-396 (2013).

5. de Graaf, M. \& Fouchier, R. A. M. Role of receptor binding specificity in influenza A virus transmission and pathogenesis. EMBO J. 33, 823-841 (2014).

6. Long, J. S., Mistry, B., Haslam, S. M. \& Barclay, W. S. Host and viral determinants of influenza A virus species specificity. Nat. Rev. Microbiol. 17, 67-81 (2019).

7. Gambaryan, A. S. \& Matrosovich, M. N. What adaptive changes in hemagglutinin and neuraminidase are necessary for emergence of pandemic influenza virus from its avian precursor? Biochem. 80, 872-880 (2015).

8. McAuley, J. L., Gilbertson, B. P., Trifkovic, S., Brown, L. E. \& McKimm-Breschkin, J. L. Influenza Virus Neuraminidase Structure and Functions. Front. Microbiol. 10, 39 (2019). 
9. Du, W., Guo, H., Nijman, V. S., Doedt, J., van der Vries, E., van der Lee, J., Li, Z., Boons, G. J., van Kuppeveld, F. J. M., de Vries, E., Matrosovich, M. \& de Haan, C. A. M. The 2nd sialic acid-binding site of influenza a virus neuraminidase is an important determinant of the hemagglutinin-neuraminidase-receptor balance. PLoS Pathog. 15, 1-24 (2019).

10. Wagner, R., Matrosovich, M. \& Klenk, H.-D. Functional balance between haemagglutinin and neuraminidase in influenza virus infections. Rev. Med. Virol. 12, 159-166 (2002).

11. de Vries, E., Du, W., Guo, H. \& de Haan, C. A. M. Influenza A Virus HemagglutininNeuraminidase-Receptor Balance: Preserving Virus Motility. Trends Microbiol. 28, 5767 (2020).

12. Neumann, G. \& Kawaoka, Y. Transmission of influenza A viruses. Virology 479-480, 234246 (2015).

13. Kobasa, D., Kodihalli, S., Luo, M., Castrucci, M. R., Donatelli, I., Suzuki, Y., Suzuki, T. \& Kawaoka, Y. Amino acid residues contributing to the substrate specificity of the influenza A virus neuraminidase. J. Virol. 73, 6743-6751 (1999).

14. Yang, X., Steukers, L., Forier, K., Xiong, R., Braeckmans, K., Van Reeth, K. \& Nauwynck, H. A Beneficiary Role for Neuraminidase in Influenza Virus Penetration through the Respiratory Mucus. PLoS One 9, e110026 (2014).

15. Cohen, M., Zhang, X.-Q., Senaati, H. P., Chen, H.-W., Varki, N. M., Schooley, R. T. \& Gagneux, P. Influenza A penetrates host mucus by cleaving sialic acids with neuraminidase. Virol. J. 10, 321 (2013).

16. Vahey, M. D. \& Fletcher, D. A. Influenza A virus surface proteins are organized to help penetrate host mucus. elife 8, 1-24 (2019).

17. Guo, H., Rabouw, H., Slomp, A., Dai, M., van der Vegt, F., van Lent, J. W. M., McBride, R., Paulson, J. C., de Groot, R. J., van Kuppeveld, F. J. M., de Vries, E. \& de Haan, C. A. M. Kinetic analysis of the influenza A virus HA/NA balance reveals contribution of NA to virus-receptor binding and NA-dependent rolling on receptor-containing surfaces. PLoS Pathog. 14, e1007233 (2018).

18. Influenza (Seasonal). WHO (2018). at <https://www.who.int/en/news-room/factsheets/detail/influenza-(seasonal)>

19. Influenza (Avian and other zoonotic). WHO (2018). at <https://www.who.int/newsroom/fact-sheets/detail/influenza-(avian-and-other-zoonotic)>

20. Ohuchi, M., Asaoka, N., Sakai, T. \& Ohuchi, R. Roles of neuraminidase in the initial stage of influenza virus infection. Microbes Infect. 8, 1287-1293 (2006).

21. Vachieri, S. G., Xiong, X., Collins, P. J., Walker, P. a, Martin, S. R., Haire, L. F., Zhang, Y., McCauley, J. W., Gamblin, S. J. \& Skehel, J. J. Receptor binding by H10 influenza viruses. Nature 511, 475-477 (2014).

22. Yen, H.-L., Liang, C.-H., Wu, C.-Y., Forrest, H. L., Ferguson, A., Choy, K.-T., Jones, J., Wong, D. D.-Y., Cheung, P. P.-H., Hsu, C.-H., Li, O. T., Yuen, K. M., Chan, R. W. Y., Poon, L. L. M. M., Chan, M. C. W. W., Nicholls, J. M., Krauss, S., Wong, C.-H., Guan, Y., Webster, R. G., Webby, R. J. \& Peiris, M. Hemagglutinin-neuraminidase balance confers respiratorydroplet transmissibility of the pandemic H1N1 influenza virus in ferrets. Proc. Natl. Acad. Sci. U.S.A. 108, 14264-14269 (2011). 


\section{Chapter 6}

23. Stevens, J., Blixt, O., Tumpey, T. M., Taubenberger, J. K., Paulson, J. C. \& Wilson, I. A. Structure and Receptor Specificity of the Hemagglutinin from an H5N1 Influenza Virus. Science 312, 404-410 (2006).

24. Di lorio, D., Verheijden, M. L., van der Vries, E., Jonkheijm, P. \& Huskens, J. Weak Multivalent Binding of Influenza Hemagglutinin Nanoparticles at a SialoglycanFunctionalized Supported Lipid Bilayer. ACS Nano 13, 3413-3423 (2019).

25. Di lorio, D. Designer Surfaces for the Quantification of Multivalent Biological Interactions. (2019). doi:10.3990/1.9789036548199

26. Müller, M., Lauster, D., Wildenauer, H. H. K., Herrmann, A. \& Block, S. Mobility-Based Quantification of Multivalent Virus-Receptor Interactions: New Insights into Influenza A Virus Binding Mode. Nano Lett. 19, 1875-1882 (2019).

27. Martinez-Veracoechea, F. J. \& Frenkel, D. Designing super selectivity in multivalent nanoparticle binding. Proc. Natl. Acad. Sci. U.S.A. 108, 10963-10968 (2011).

28. Curk, T. \& Tito, N. B. First-order 'hyper-selective' binding transition of multivalent particles under force. J. Phys. Condens. Matter 32, 214002 (2020).

29. Dubacheva, G. V., Curk, T., Auzély-Velty, R., Frenkel, D. \& Richter, R. P. Designing multivalent probes for tunable superselective targeting. Proc. Natl. Acad. Sci. U.S.A. 112, 5579-5584 (2015).

30. Dubacheva, G. V., Curk, T., Frenkel, D. \& Richter, R. P. Multivalent Recognition at Fluid Surfaces: The Interplay of Receptor Clustering and Superselectivity. J. Am. Chem. Soc. 141, 2577-2588 (2019).

31. Albertazzi, L., Martinez-Veracoechea, F. J., Leenders, C. M. A., Voets, I. K., Frenkel, D. \& Meijer, E. W. Spatiotemporal control and superselectivity in supramolecular polymers using multivalency. Proc. Natl. Acad. Sci. U.S.A. 110, 12203-12208 (2013).

32. Tito, N. B. \& Frenkel, D. Optimizing the selectivity of surface-adsorbing multivalent polymers. Macromolecules 47, 7496-7509 (2014).

33. Scheepers, M. R. W., van IJzendoorn, L. J. \& Prins, M. W. J. Multivalent weak interactions enhance selectivity of interparticle binding. Proc. Natl. Acad. Sci. U.S.A. 117, 2269022697 (2020).

34. Reiter-Scherer, V., Cuellar-Camacho, J. L., Bhatia, S., Haag, R., Herrmann, A., Lauster, D. \& Rabe, J. P. Force Spectroscopy Shows Dynamic Binding of Influenza Hemagglutinin and Neuraminidase to Sialic Acid. Biophys. J. 116, 1037-1048 (2019).

35. Cuellar-Camacho, J. L., Bhatia, S., Reiter-Scherer, V., Lauster, D., Liese, S., Rabe, J. P., Herrmann, A. \& Haag, R. Quantification of Multivalent Interactions between Sialic Acid and Influenza A Virus Spike Proteins by Single-Molecule Force Spectroscopy. J. Am. Chem. Soc. 142, 12181-12192 (2020).

36. Seladi-Schulman, J., Steel, J. \& Lowen, A. C. Spherical Influenza Viruses Have a Fitness Advantage in Embryonated Eggs, while Filament-Producing Strains Are Selected In Vivo. J. Virol. 87, 13343-13353 (2013).

37. Harris, A., Cardone, G., Winkler, D. C., Heymann, J. B., Brecher, M., White, J. M. \& Steven, A. C. Influenza virus pleiomorphy characterized by cryoelectron tomography. Proc. Natl. Acad. Sci. U.S.A. 103, 19123-19127 (2006). 
38. Vahey, M. D. \& Fletcher, D. A. Low-Fidelity Assembly of Influenza A Virus Promotes Escape from Host Cells. Cell 176, 281-294 (2019).

39. Rimmelzwaan, G. F., Baars, M., Claas, E. C. J. \& Osterhaus, A. D. M. E. Comparison of RNA hybridization, hemagglutination assay, titration of infectious virus and immunofluorescence as methods for monitoring influenza virus replication in vitro. J. Virol. Methods 74, 57-66 (1998).

40. Dubacheva, G. V., Araya-Callis, C., Geert Volbeda, A., Fairhead, M., Codée, J., Howarth, M. \& Richter, R. P. Controlling Multivalent Binding through Surface Chemistry: Model Study on Streptavidin. J. Am. Chem. Soc. 139, 4157-4167 (2017).

41. Nagle, J. F. \& Tristram-Nagle, S. Structure of lipid bilayers. Biochim. Biophys. Acta-Rev. Biomembr. 1469, 159-195 (2000). 


\section{Chapter 7}

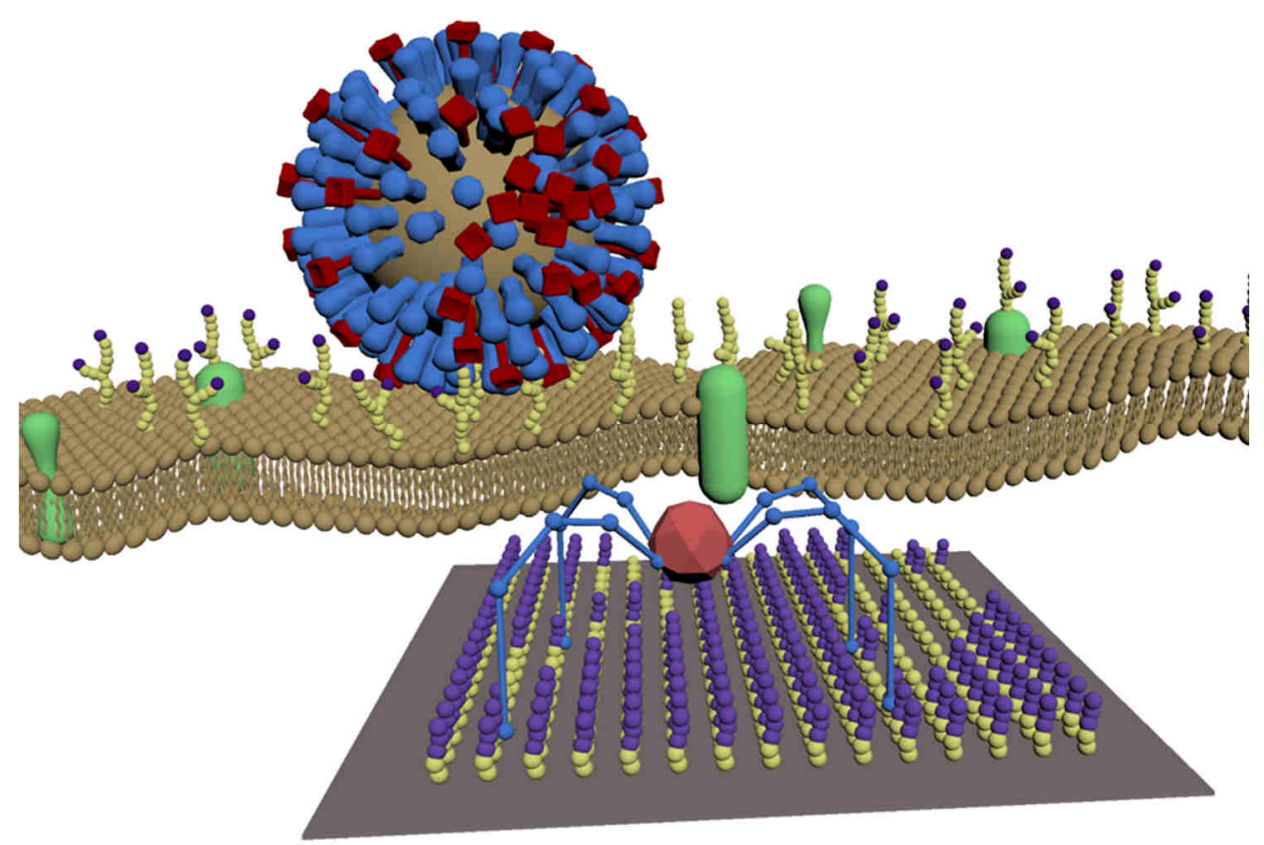

\section{Highlights}

- The neuraminidase on the surface of influenza virus cleaves its receptors

- Molecular spiders are a type of molecular walkers that move directionally by cleaving their receptors

- Influenza viruses can move over receptor-displaying surfaces

- We argue that influenza viruses behave similarly to molecular spiders 


\section{Influenza as a molecular walker}

The surface of the influenza virus is decorated with the receptor-binding protein hemagglutinin (HA) and the receptor-cleaving enzyme neuraminidase (NA). HA is responsible for host cell recognition, while NA prevents aggregation and entrapment, but the intricate mechanism of how the functions of these glycoproteins cooperate and how they are regulated by mutational responses to environmental pressures remains unclear. Recently, several groups have described the motion of influenza over surfaces and reported that this motion is inhibited by NA inhibitors. We argue that the motion of influenza resembles the motility of artificial receptor-cleaving particles called "molecular spiders". The cleaving of receptors by this type of molecular walkers leads to self-avoiding motion across a surface. When the binding and cleaving rates of molecular spiders are balanced, they move both rapidly and efficiently. The studies of molecular spiders offer new insights into the functional balance of HA and NA, but they do not address the asymmetric distribution of HA and NA on the surface of influenza. We propose that receptor-cleaving molecular walkers could play an important role in the further investigation of the motility of influenza viruses.

Part of this chapter has been published in:

Hamming, P. H. *, Overeem, N. J.*, Huskens, J., Chem. Sci. 2020, 11, 27-36.

*Authors contributed equally. 


\subsection{Introduction}

Influenza is among the most common diseases in the world. The disease and mortality of seasonal outbreaks and the deadliness of the rarer pandemic outbreaks have made influenza a prime target of virology. Its facile adaptation to a range of different hosts and its rapid evolution under antigenic pressure originate from two cooperating glycoproteins, the receptor-binding hemagglutinin (HA) and the receptor-cleaving enzyme neuraminidase (NA), and from the rapid mutations that occur in the genetic material that encodes for these proteins. ${ }^{1,2} \mathrm{HA}$ and NA make up the characteristic 'spikes', and are jointly responsible for the surface interactions of the virus with a host cell and for its passage through the mucus layer that protects the host cell. ${ }^{1,2}$ These interactions are far more complex than an ordinary receptor-ligand equilibrium and are only partially understood.

It is widely accepted that the receptor-binding function of $\mathrm{HA}$ and the receptor cleaving function of NA must be balanced for successful infection. ${ }^{3-5}$ This functional balance between HA and NA is reflected in the evolution of HA and NA. ${ }^{6-10}$ It has therefore been suggested that all changes in activity of HA or NA must be followed by an adjustment of the activity of the other to maintain a functional balance. ${ }^{6,7,11,12}$ In vivo studies have shown that lower NA activity leads to less efficient virus replication, but stronger binding by HA can have the same effect. ${ }^{13}$ It is believed that the role of NA in this balance is to prevent aggregation of the virus and entrapment of progeny viruses on the surface of host cells. ${ }^{1,3,14}$

Recently, several groups have described a new function of NA in imparting motility of the virus on a surface. ${ }^{15-18}$ Sakai et al. were the first to report that the motion of influenza over a surface is NA-dependent. ${ }^{15}$ They also showed that this motility increased cellular uptake of the virus. De Haan et al. found that the receptor-cleaving activity of a few adsorbed viruses is enough to prevent adsorption of new viruses and proposed that the viruses roll over the surface while cleaving off the receptors across the path they follow. ${ }^{16}$ Vahey and Fletcher found that the organization of HA and NA on filamentous viruses imparts directionality to their motion, and these viruses crawl rather than roll. ${ }^{18}$ These new observations call for a model that can account for this motility and has predictive power. 
Surface-confined motility is not new, neither in biological nor in synthetic systems. In biology, the most famous examples are the kinesin and myosin $\mathrm{V}$ motor enzymes, which transport cargo unidirectionally along microtubules and actin filaments. ${ }^{19-21}$ Vogel et al. showed that kinesin immobilized on a surface could impart motility onto microtubules in a synthetic environment as well. ${ }^{22}$ Synthetic systems which aim to achieve motion over a surface or track are called 'molecular walkers'. ${ }^{23}$ In the simplest form a molecular walker is a biped with feet that can bind to and release from a surface sequentially, and it will act like a molecular walker for as long as at least one foot remains attached to the surface. ${ }^{24}$ Its movement depends on Brownian motion and will therefore be diffusive and non-directional, unless it can move over a gradient or is inhibited in one direction by ratcheting. ${ }^{25,26}$

Mimicking directional motion as shown by kinesin and myosin $\mathrm{V}$ requires the walker to overcome Brownian motion and requires energy input. ${ }^{27}$ The natural motor enzymes use ATP as fuel, whereas synthetic systems have a wider array of possible energy sources. ${ }^{28,29}$ The first molecular walker used DNA strands as fuel, quickly followed by an example that used ATP as fuel. ${ }^{23,30}$ Instead of using the consumption of a chemical fuel to impose a strict directionality in each individual step, overall directionality can be attained when the direction of the steps is biased, for example using an enzyme with a chiral preference to cleave the back leg. ${ }^{31}$ For a comprehensive review of different walker designs, energy sources and mechanisms, see: Leigh et al. ${ }^{29}$

Stojanovic et al. introduced a class of molecular walkers, called 'molecular spiders', that use their receptors as fuel. ${ }^{32} \mathrm{~A}$ molecular spider consists of a body with multiple catalytically active legs that cleave the receptors to which they bind. The cleaving of its receptors is the energy source for biased motion away from their starting position, leading to self-avoiding walking. Its essential design is simple enough to allow systematic study, both experimentally and in silico, while their receptor-cleaving dependent motion may be comparable to that of influenza, as will be explored below.

In this chapter, it is shown that the combined roles of HA and NA in the surfacebound motility of influenza can be understood by looking at receptor-cleaving molecular walkers. We first explain the mechanism of surface-bound motion in receptor-cleaving molecular walkers. Then we describe how this mechanism leads to faster-than-diffusive and self-avoiding motion and how these 
properties are influenced by the design of the walker. The roles of the HA and NA proteins are discussed, as well as their presentation on the surface of influenza. We comprehensively review the proposed mechanisms that have been published about influenza movement on cell surfaces. We argue that both the "rolling" and the "Brownian ratchet" mechanism requires collaborative interactions of HA and NA that make influenza a molecular walker and propose that both models can explain how influenza can efficiently cross the mucus layer. We then discuss how properties of molecular spiders may be favorable for influenza in avoiding clearance by the mucus and in finding a suitable site for host cell infection. After that, we discuss the similarities and differences in the motility of molecular spiders and influenza. Finally, we indicate research directions for molecular walkers that have high relevance for understanding influenza. We will not discuss the role of HA and NA inside the cell, as their role in the complete cycle of infection has been described elsewhere. ${ }^{33,34}$

\subsection{Molecular spiders and superdiffusive walking}

Molecular spiders interact with a surface through their legs. All legs are identical and cleave the sites to which they bind. ${ }^{32,35,36}$ Experimental setups of molecular spiders generally use DNA on the surface and catalytically active DNA (DNAzymes) as legs. ${ }^{32,35,37,38}$ After binding through base pairing, the DNA on the surface is cleaved by the DNAzyme, resulting in a shorter DNA strand (Figure 7.1a). The DNAzymes are still able to bind to these shorter strands but have a diminished affinity and residence time.

When a molecular spider is on the boundary between uncleaved substrates and cleaved products, it experiences a gradient in affinity and residence times, leading to a directional bias towards the uncleaved substrates. The cleaving of receptors acts as a fuel to continually create the gradient. With each step of the spider, the boundary shifts one step as well (Figure 7.1b). For as long as the spider remains at this self-propelling boundary, it moves away from the starting position faster than it would by normal diffusion. This mode of motion is called superdiffusivity. ${ }^{36}$ The directionality is temporarily lost if the spider moves a step away from the boundary (Figure 7.1c). The overall bias depends therefore primarily on the chance that the spider remains at the moving boundary. 


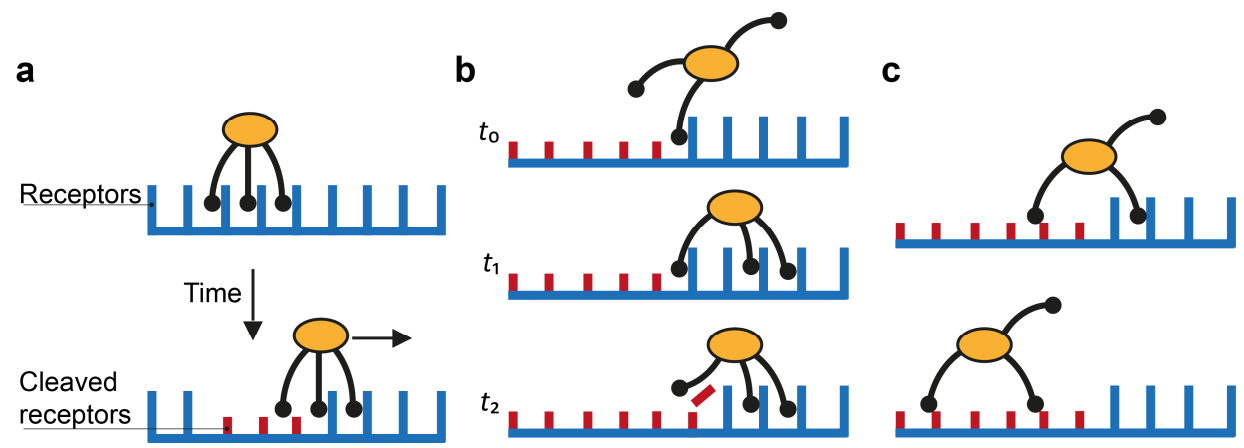

Figure 7.1| Mechanism for directional motility in molecular spiders. ${ }^{36,39}$ a) A molecular spider consists of a rigid body with several legs. The legs bind to receptors on a surface, cleaving them as they go. The legs can bind to a cleaved receptor, but have a lower residence time. b) The difference in residence time can lead to a bias in movement. At $t_{0}$, the spider is attached at the boundary between fresh and cleaved receptors with one leg. At $t_{1}$, the legs are more likely to be bound to fresh than to cleaved receptors due to the difference in residence times. At $t_{2}$, the first leg detaches and leaves a cleaved receptor behind, shifting the boundary. c) The spider is either at the boundary and moving with a bias towards fresh receptors, or the spider is in a patch of cleaved receptors where all legs have low residence time and diffusion is fast.

The superdiffusive motion of a molecular spider is weakly self-avoiding; it is possible for the spider to revisit a site, but it will spend most of its time visiting new sites. Some molecular spiders cannot revisit former sites at all and are therefore strictly self-avoiding; these are also called 'burnt-bridges' walkers. ${ }^{29}$ When a spider leaves the boundary between cleaved and uncleaved receptors or reaches a previously visited area, it undergoes non-directional diffusive motion over the surface. The low residence time of the legs on cleaved receptors results in fast diffusion of the spider. The spider thus spends only limited time in this diffusive state and quickly returns to a boundary where it again undergoes biased motion away from the cleaved receptors. This weakly self-avoiding walk is a more efficient way of probing an area than diffusion. ${ }^{36,40}$

The superdiffusivity of a molecular spider is determined by how well it recognizes the gradient at the boundary between cleaved and uncleaved receptors. The state of a spider on this boundary is defined by the length of its legs $L$ and the number of legs $n$ (Figure 7.2a). The superdiffusive behavior of a spider emerges from its multivalent character, because one-legged spiders cannot recognize a gradient. ${ }^{41}$ Whether additional legs increase superdiffusivity 
depends on the length of the legs. The superdiffusive behavior of molecular spiders with short legs benefits from a high number of legs ${ }^{40}$ whereas a high number of legs decreases the superdiffusivity of molecular spiders with long and flexible legs. ${ }^{42}$

a

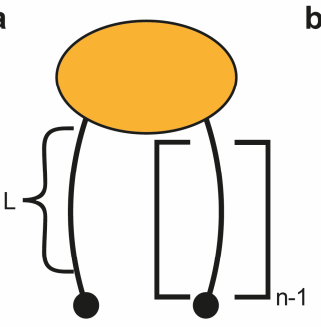

c
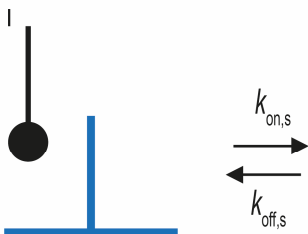

b
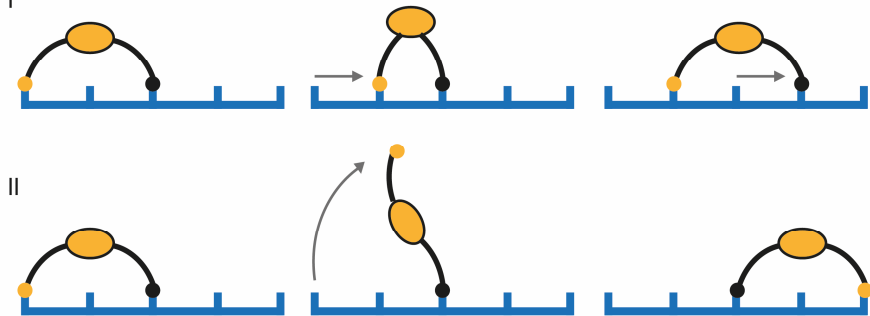

II

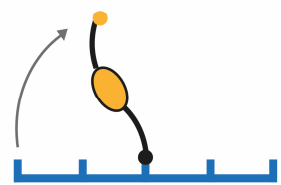

III

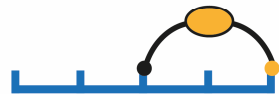

IV

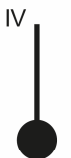

Figure 7.2 | Main design parameters in molecular spiders. a) The number of legs $(n)$ of a spider and the length of each leg $(L)$ define the boundary state of the spider. b) Molecular spiders can walk using an inchworm (I) or hand-over-hand (II) motif. c) The kinetics of interaction between the substrate and an individual leg.

The maximum size of a step is determined by the length of the legs of a molecular spider and by its gait. Molecular spiders tend to move faster if they use a hand-over-hand or rolling motion than if they use inchworm motion. ${ }^{38} \mathrm{It}$ is faster because a hand-over-hand gait limits the step size to how far the spider can stretch, whereas the inchworm gait limits the step size to the farthest point that is already attached (Figure $7.2 \mathrm{~b}$ ).

The rate of each step is determined by the kinetics of the catalytically active legs and by the dissociation rate of a leg from a cleaved receptor (Figure 7.2c). Stronger binding of a leg towards an uncleaved receptor means a lower Michaelis-Menten constant and can therefore increase the speed of walking. Less intuitive is that stronger binding to both the uncleaved and the cleaved receptors can increase the superdiffusivity of a spider, not only compensating the slower release of its legs but even increasing its velocity. ${ }^{35,38,43}$ 
To maintain a molecular walk over longer distances, a functional balance is needed between the cleaving activity and the binding activity of a molecular spider. A higher cleaving activity leads to faster motion, but if its cleaving activity exceeds its binding activity the spider dissociates. ${ }^{32,42}$

For the exploration of larger surfaces, dissociation of a molecular spider can even be favorable. If a spider finds itself in an area that was visited before, the low residence time of legs on cleaved receptors increases the probability that all legs are unbound at the same time, releasing the spider from the surface. ${ }^{36,38}$ After dissociation, the spider can diffuse through the solution for some distance and associate again with a higher association constant for unvisited areas. This type of motion could be regarded as a "self-avoiding hop", so that molecular spiders are weakly self-avoiding over multiple length scales.

\subsection{Motility of influenza virus}

Of the two glycoproteins on the surface of influenza virus that are involved in host cell binding, $\mathrm{HA}$ is the most abundant. HA is a trimeric protein that can bind sialic acid-terminated glycans with millimolar affinities (Figure 7.3a).,54 The HA conveys hostcell specificity to the virus by binding with greater affinity to a sialic acid that has either a 2,3-linkage to a neighboring galactose (2,3-SLN), which is more abundant in avian intestines, or a 2,6-linkage (2,6-SLN), which is more abundant in the human upper respiratory tract. ${ }^{1,34}$ This specificity is amplified by the multivalent presentation of HA on the virus so that small differences in the affinity of HA lead to major differences in affinity of the virus. $^{44}$

The secondary glycoprotein, NA, is a tetrameric enzyme that binds sialic acidterminated glycans and then hydrolyses the bond between the sialic acid and galactose units (Figure 7.3b). NA typically has a Michaelis-Menten constant $K_{m}$ in the low millimolar range and a turnover number $k_{c a t}$ of several $10 \mathrm{~s}$ per second. ${ }^{5,45}$ In some virus strains $k_{\text {cat }}$ is reduced to tenths per second. ${ }^{46}$ This magnitude of $K_{m}$ means that the initial contribution of NA to the binding of a virus is comparable to that of HA. The enzyme-substrate complex is, however, short lived as its lifetime is not greater than $k_{\text {cat }}{ }^{-1}$. Cleaving off the terminal sialic acid by NA reduces the affinity of HA to the glycan by more than 10 -fold. ${ }^{47,48}$ 
a

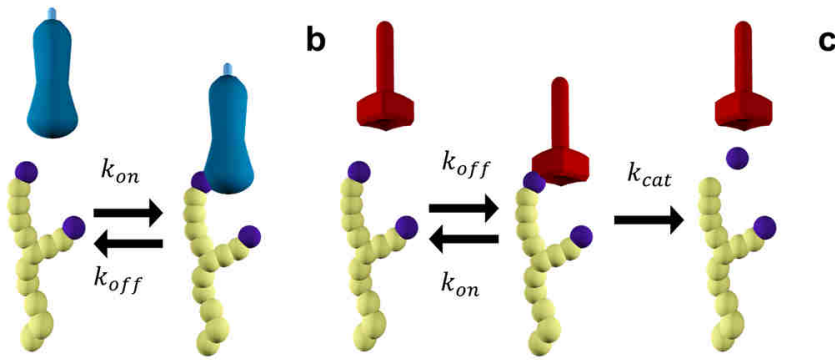

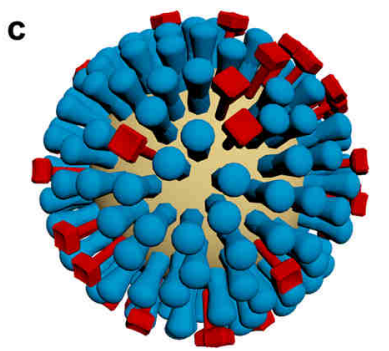

Figure 7.3 Structure and interactions of influenza virus a) Hemagglutinin (HA) is a trimeric protein that binds to sialic acid-terminated glycans in a reversible manner. b) Neuraminidase (NA) is a tetrameric protein that binds and cleaves sialic acid-terminated glycans. c) HA and NA are present on the surface of influenza in high copy numbers, approximately in a 6:1 ratio.

The HA and NA are presented on the surface of influenza in high copy numbers in an approximately 6:1 ratio (Figure 7.3c)..$^{49}$ The virus is approximately $100 \mathrm{~nm}$ in diameter and can be almost spherical or filamentous, with lengths up to a micrometer. HA and NA are densely packed on the surface, but not entirely random. Patches or even complete separation of HA and NA into domains have been reported. ${ }^{18,49,50}$ This distribution likely originates from an interplay between entropic factors and differences in the preferred membrane curvature between $\mathrm{HA}$ and NA. ${ }^{50-53}$ It is likely that the organization of HA and NA has an influence on the way their functions cooperate.

Motility of influenza was first reported by Sakai et al..$^{15}$ They tracked the motion of influenza viruses over fetuin-modified glass with total internal reflection fluorescence microscopy (TIRF). When adding an NA inhibitor, the movement of the viruses was completely blocked. They concluded that the virus moves over a cell surface not by lateral diffusion of the virus along receptor sites, but by NA-initiated exchange of receptors from one binding pocket to the next. They distinguished two modes of movement: a slower and more frequent "crawling", and a faster and rarer "gliding" (Figure 7.4). Lower NA activity in mutant viruses led to slower motility and lower occurrence of gliding steps. The gliding steps were also shorter. Therefore, they concluded that NA is not only necessary to initiate movement, but also to sustain gliding steps. The NAdependent motion of influenza was not only demonstrated on artificial surfaces, but also on live cells. Adding an NA inhibitor or using a mutant with less active NA both led to decreased endocytosis of the viruses. 
In a kinetic analysis of the binding of influenza HA and NA with biolayer interferometry (BLI), De Haan et al. found that the cleaving of receptors by adsorbed viruses was far more complete than suggested by Figure $7.4 \mathrm{c} .{ }^{16}$ They allowed various concentrations of influenza to bind to a receptor-functionalized sensor in the presence of NA inhibitor, followed by transfer of the sensor with adhering viruses to a buffer solution. If the buffer also contained NA inhibitor, the viruses remained adhering, but without inhibitor, they dissociated completely. When a lower amount of virus was adsorbed, the viruses dissociated more slowly. Then they regenerated the sensors at $\mathrm{pH} 2$, removing all viruses, but leaving the receptors on the sensors. The regenerated sensors were subsequently allowed to re-bind viruses at the maximum concentration. While the sensors that were placed in buffer with NA inhibitor were unaffected, the sensors where the virus was dissociated could bind significantly fewer new viruses.
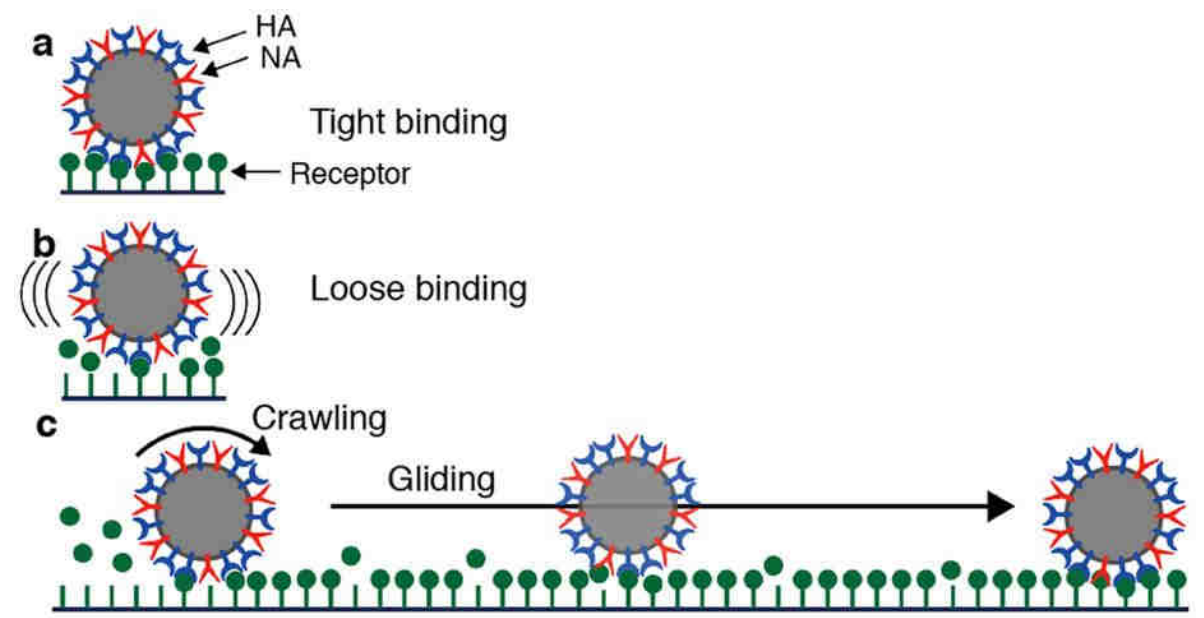

Figure 7.4| Mechanism for virus motion proposed by Sakai et al. ${ }^{15}$ a) Influenza binds tightly to a surface with multiple HA-receptor interactions. b) NA cleaves receptors, which decreases the number of interactions and initiates motility. c) A loosely attached virus performs crawling and gliding motions by iterative association and dissociation of HA-receptor interactions, until it reaches a site where it can form multiple interactions and again bind tightly to the surface. Reprinted from ref. ${ }^{15}$, with permission from Springer Nature, licensed under CC BY 4.0. 


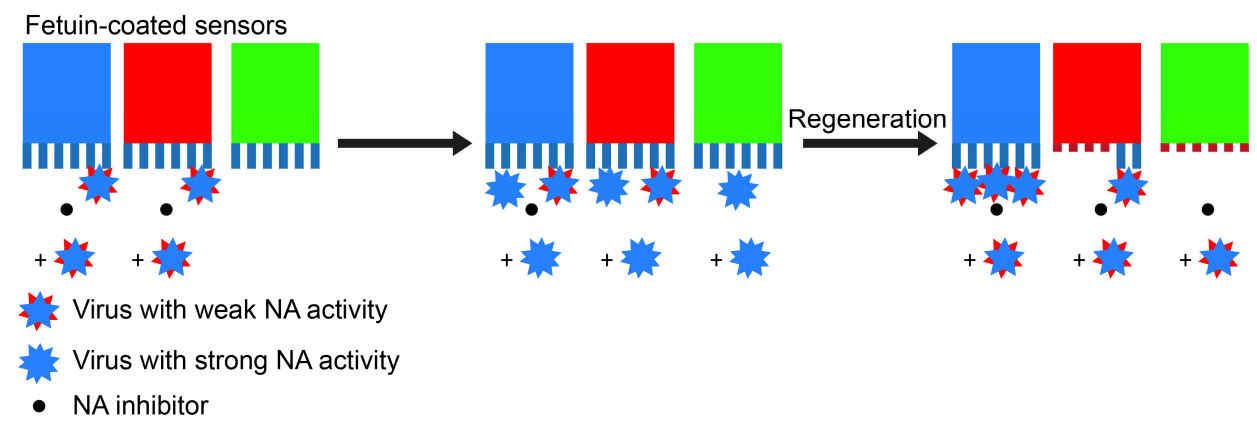

Figure 7.5 Motility of influenza is driven by NA activity. De Haan et al. partially blocked receptors on BLI sensors with viruses that had inactive NA and then exposed the sensors to a virus with active NA. ${ }^{16}$ One sensor (in blue) was protected with the NA inhibitor oseltamivir carboxylate (OC), one sensor (in red) was only locally blocked by the NA-inactive virus, and one sensor (in green) was left fully unprotected. After regeneration of the sensors, exposure to new virus showed that receptors were cleaved from all unprotected areas. Adapted from ref. ${ }^{16}$ with permission from Public Library of Sciences, licensed under CC BY 4.0.

To test whether the deactivation of the sensors was due to receptor cleavage by NA, De Haan et al. blocked two sensors partly with a virus with inactive NA (Figure 7.5). Then they incubated the partly blocked sensors and a third sensor with a different virus that had active NA, with NA inhibitor present at one of the two blocked sensors. Then they regenerated the sensors and allowed the virus with inactive NA to bind on all three sensors. Binding was uninhibited at the sensor that was protected with NA inhibitor, partly inhibited at the sensor that was partly blocked, and fully inhibited at the exposed sensor. With these two experiments they showed that NA-dependent "rolling" of a low amount of virus could cleave receptors from a large surface area.

Vahey and Fletcher showed that the asymmetric distribution of HA and NA on filamentous viruses imparted a directional bias in over several micrometers. ${ }^{18}$ Filamentous viruses are more common in clinical isolates, whereas laboratorygrown strains often produce more spherical viruses. ${ }^{52,54}$ Vahey and Fletcher hypothesized that the filamentous shape together with non-uniform distribution of HA and NA promote efficient penetration of viruses through mucus. They labelled the HA, NA and nucleoprotein (NP) of a filamentous virus and showed that HA was more or less homogenously distributed, NP was clustered at one pole and NA was more abundant at the same pole (Figure 7.6). 


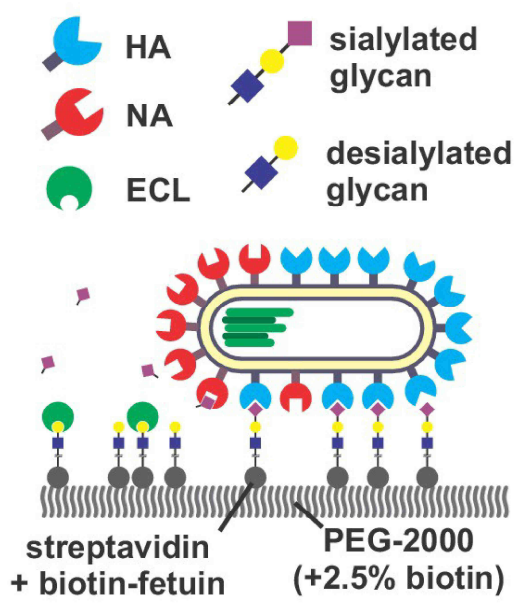

Figure 7.21 Motility through asymmetric organization in filamentous viruses. By labelling $\mathrm{HA}, \mathrm{NA}$ and cleaved receptors, Vahey and Fletcher showed that the asymmetric organization of HA and NA imparts directional motility in filamentous viruses. ${ }^{18}$ Reprinted from ref. ${ }^{18}$ with permission from elife Sciences Publications, licensed under CC BY 4.0

Tracking the viruses with TIRF and imaging with super-resolution microscopy, they showed that the virus moved directionally away from the NA-rich pole. By labeling cleaved receptors with the fluorescent lectin ECL, they showed that receptors were cleaved in the path of the virus. They posed that the cleaving of receptors functions as a Brownian ratchet by restricting backward motion. To predict the effect of the diffusion coefficient of receptors on the mean square displacement of viruses, they made a computational model. They found that the velocity of viruses is the highest when the diffusion coefficient of receptors is small, but not zero. The values for the diffusion coefficient where motility is fastest are consistent with the diffusion coefficients of sialic acid in mucus. They cultured mucus-secreting cells to test whether the virus could also move through a three-dimensional environment and observed tracks similar to those observed on two-dimensional surfaces.

Block et al. also tracked influenza viruses, but with the aim of using its mobility to quantify the number of receptor-ligand interactions. ${ }^{17}$ They tracked labelled influenza viruses with TIRF on a supported lipid bilayer (SLB) containing glycolipid receptors (Figure 7.7). Because multivalent binding decreases the dissociation rate constant $k_{\text {off }}$ and the diffusion coefficient $D$, the average 
valency scales with $1 / D$. In a plot of $k_{\text {off }}$ versus $1 / D$, the effect of multivalent binding appears as an exponential decline but is actually composed of subpopulations that share the same $D$ values (Figure 7.7). Interestingly, there is a peak structure visible with elevated $k_{\text {off }}$ values for $1 / D \sim 5 \mathrm{~s} / \mu \mathrm{m}^{2}$. This peak decreases with added NA inhibitor and is therefore ascribed to receptor cleaving by NA. Block et al. claim that the lateral diffusion of the glycolipids to which influenza binds remains significantly faster than the NA-dependent motility that was reported by Sakai et al. ${ }^{15,17}$

It is interesting to note the differences in setup and observed mobility between these four publications. Both Block et al. and Sakai et al. used an egg-adapted influenza strain with HA and NA from the IAV strain Aichi/2/68 (H3N2). ${ }^{15,17}$ Vahey and Fletcher used HA and NA from A/WSN/33 (H1N1). ${ }^{18}$ De Haan et al. used the HA and NA from the same strain for its lower NA activity and used A/Puerto Rico/8/34 Mount Sinai (H1N1) to demonstrate the removal of receptors. ${ }^{16}$ In an assay of NA activity, Aichi/2 was approximately fivefold less active than WSN, which was fivefold less active than PR/8 Mt. Sinai. ${ }^{5,16}$ It is therefore remarkable that Block et al. report a motion that is up to two orders of magnitude faster than Sakai et al., whereas Vahey and Fletcher reported a motion that is an order of magnitude slower than Sakai et al. ${ }^{15,17,18}$ Unlike De Haan et al., who reported that NA contributes to the association of viruses, ${ }^{16}$ Block et al. report an increase of association rate with added NA inhibitor. ${ }^{17}$

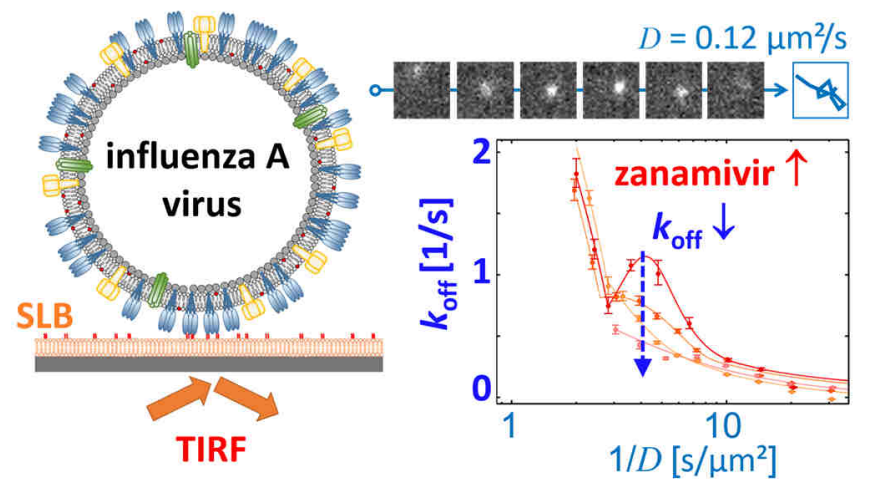

Figure 7.3| Lateral diffusion of influenza viruses on supported lipid bilayers. Block et al. tracked influenza viruses on supported lipid bilayers to quantify the number of interactions with glycolipids. ${ }^{17}$ They observed elevated $k_{\text {off }}$ values for $1 / D \sim 5 \mathrm{~s} / \mu \mathrm{m}^{2}$, which decreased with added NA inhibitor. Reprinted with permission from ref. ${ }^{17}$. Copyright 2019 American Chemical Society. 
We think it likely that the differences described above are primarily due to the different structures of the surfaces that were used in these studies. Block et al. used 1 mol\% of glycolipid GDa1 in an SLB; ${ }^{17}$ Sakai et al. used $10 \%$ fetuin with albumin adsorbed on glass; ${ }^{15}$ De Haan et al. used BLI sensors with streptavidin and biotinylated fetuin; ${ }^{16}$ and Vahey and Fletcher used a PEG2000 brush on glass with streptavidin and biotinylated fetuin. ${ }^{18}$ Sakai et al. reported an optimum in the receptor density, while Vahey and Fletcher reported an optimum in the diffusion coefficient of sialic acid. ${ }^{15,18}$ We expect that there exists also an optimum in the conformational flexibility of the receptors. These differences show that the motility of a virus depends on a combination of many properties of both the virus and the surface.

\subsection{The role of motility in host cell recognition}

Sakai et al. found that the NA-dependent motility of influenza contributes to its endocytosis, but did not study the mechanism of this contribution. ${ }^{15}$ They propose that the motility of the virus allows lateral motion even if receptors are restricted from diffusion by membrane rafts or other rigid structures. They also argue that it must play an important role in intercellular migration.

The studies of molecular spiders offer two additional possible advantages of molecular walking over random lateral diffusion. Firstly, the weakly selfavoiding walk provides a more efficient pattern to search the surface of a cell for a suitable location to bind to induce endocytosis (Figure 7.8a). Secondly, the molecular walk provides a bias towards high receptor densities (Figure 7.8b). Because endocytosis of influenza is mediated by clathrin-coated as well as noncoated pits, it is likely that influenza searches for locations where they can form. ${ }^{34,55}$ The bias towards higher receptor densities follows from the mechanism of walking and may provide a means of recognition to the virus if such locations prove to have a higher density of receptors. Higher densities are also favored by the ability of influenza to bind multiple receptors through $\mathrm{HA},{ }^{56}$ but without motility the chance of binding to an area of high receptor density remains relatively low for an individual virus. 
a

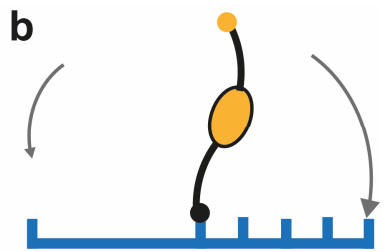

Figure 7.4| Directional walking can be a search mechanism. a) Self-avoiding walking gives rise to an efficient search pattern to find suitable sites for cell entry. b) The motility of molecular spiders is biased towards a higher density of receptors.

De Haan et al. and Vahey and Fletcher claim that the primary advantage of motility for the virus is to penetrate the mucus layer. ${ }^{16,18}$ This mucus layer consists of a dense gel of mucins that is propelled by a layer of cilia that extend from the epithelial cells in the respiratory tract (Figure 7.9a). ${ }^{57,58}$ The mucins are glycoproteins with a sugar content of $50-80 \mathrm{wt} \%$ and rich in sialic acid. ${ }^{59}$ The mucus can therefore easily entrap sialic acid-binding viruses such as influenza and clear them from the respiratory tract. ${ }^{60}$

It has been shown that influenza uses NA to cleave sialic acid when the virus passes through the mucus and thus prevents entrapment. ${ }^{61,62}$ The sialic acid in the human mucus is mostly 2,3-linked, and human-adapted viruses usually have an HA specificity for 2,6-SLN, but an NA specificity for both 2,3- and 2,6SLN. ${ }^{1,2,63,64}$ The effect of this cleaving on influenza was evidenced by an in vitro experiment where infection by a virus with weak NA activity was inhibited by human mucus, but for a virus with stronger NA activity it was not. ${ }^{65}$ It is believed that this prevention of entrapment of entering viruses in the mucus is essential for aerosol transmission between humans. ${ }^{4,66}$

Preventing entrapment and clearance of the viruses may, however, not be the only reason why this receptor cleavage is important for the virus in crossing the mucus. While the mucus layer allows diffusion of inert particles of the size of influenza virus, the periciliary layer underneath contains a mesh that is dense enough to exclude even $40 \mathrm{~nm}$ particles. ${ }^{57,60}$ This mesh is formed by mucins that are tethered in a bottle brush-like structure to the cilia. ${ }^{67}$ It is therefore likely that influenza, instead of relying on diffusion that is inhibited by interactions with sialic acid, must use an active motion to cross over the mucins and cilia towards the surface of the respiratory epithelial cells (Figure 7.9b). 


\section{a}

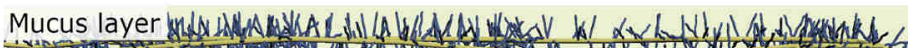

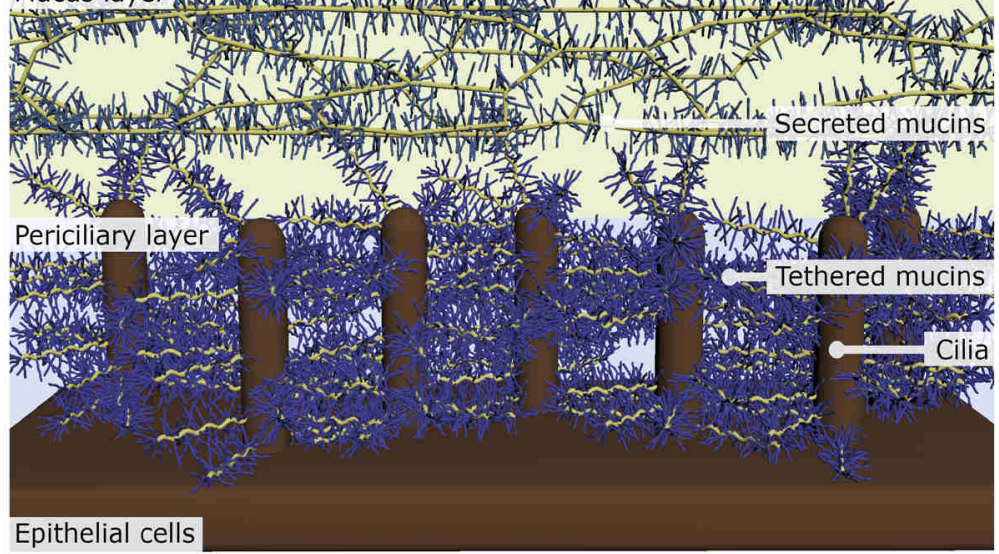

b

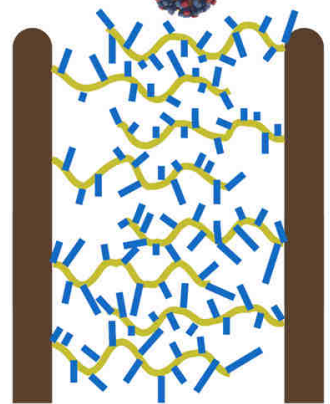

I. Repulsion

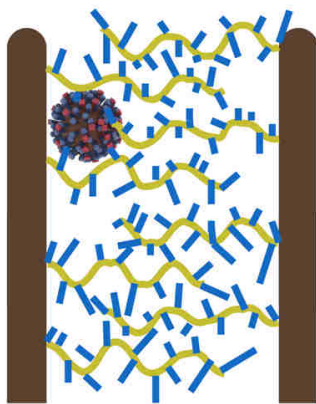

II. Entrapment

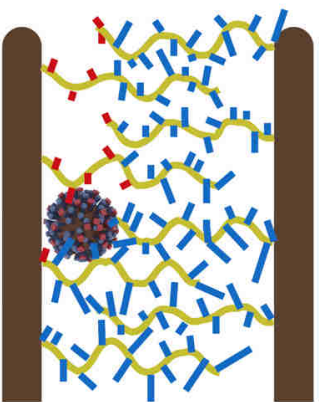

III. Walking

Figure 7.5 I Interactions of influenza virus with the mucus. a) The structure of the mucus in human airways as proposed by Rubinstein et $a l^{.57}$ b) The function of molecular walking of influenza in crossing the mucus. I. When there is no interaction, particles are repelled by the charged brush. II. Particles that have an affinity for sialic acid are entrapped. III. Influenza, which binds and cleaves sialic acid, can walk through the mucus.

\subsection{Lessons from molecular walkers for influenza}

We have described how directional motion emerges both in molecular spiders and influenza from their receptor cleaving properties. In this section we first address the lessons from molecular spiders that may apply to influenza and how they can help understand the changes that influenza viruses undergo in their adaptation. Thereafter, we discuss the properties of the receptor-ligand 
interactions of influenza that are not addressed by molecular spiders and how they may be addressed by other molecular walkers.

Probably the most interesting lesson from molecular spiders is that a higher cleaving activity leads to faster motion, but if the cleaving activity exceeds the binding activity the spider dissociates. ${ }^{32,42}$ This suggests that if there is a selective pressure on an influenza population to develop increasing motility, both the NA activity and the affinity of HA will increase. Conversely, if the affinity of HA decreases, there will be a selective pressure to decrease its NA activity as well. This co-optimization may offer an explanation why influenza acquired both stronger binding to, and faster cleaving of, 2,6-SLN during circulation in the human population. ${ }^{7}$

Another trend that was reported for molecular spiders is that the superdiffusivity of a spider with short legs increases with the number of legs. ${ }^{40}$ The HA and NA of influenza are too short and rigid to reach behind another trimer or tetramer, so it is likely that the superdiffusivity of influenza can benefit from the high number and density of glycoproteins on its surface. Because filamentous viruses have more interactions with the cell surface because of their larger contact area, their superdiffusivity may be increased over spherical viruses.

Different from molecular spiders is the gait of influenza. Molecular spiders move faster by rolling than by inchworm motion, ${ }^{38}$ but Vahey and Fletcher demonstrated that the motion of influenza is more inchworm-like. ${ }^{18}$ It remains, however, interesting to see if spherical influenza viruses would move like the filamentous viruses of Vahey and Fletcher or would shift to rolling.

The main reason why Vahey and Fletcher found an inchworm gait is that HA and NA are organized in trimers and tetramers that are asymmetrically distributed on the surface of the virus, ${ }^{18}$ unlike molecular spiders which have identical legs that perform both the binding and cleaving actions. It would be interesting to see how a molecular walker would behave where only a fraction of the legs is catalytically active and how an asymmetric distribution of these legs would affect its behavior.

In contrast with Vahey's and Fletcher's observations for influenza, molecular spiders do not need a separation of their binding and cleaving action for 
directional motion. This separation of actions may benefit influenza by allowing adjustment of its functional balance of binding and cleaving through independent variation of the cleaving activity and the binding activity of NA, the affinity of HA, and the ratio of HA to NA copy numbers. Indeed, some H3N2 strains have adapted in the human population by developing binding through their NA with almost undetectable HA binding. ${ }^{46,68}$

Large rolling motors developed by Salaita et al. share their walking mechanism with molecular spiders, although they are powered by enzymes in solution. ${ }^{69,70}$ These rolling motors demonstrate that receptor-cleaving molecular walkers of micrometer size can be propelled with high speed over long distances due to their polyvalency. Interestingly, if these particles form dimers, they roll over their shared longitudinal axis, ${ }^{69}$ in contrast with influenza's inchworm motion. ${ }^{18}$ This may indicate that the organization of HA and NA on influenza dictates its gait.

Perhaps the most important aspect of influenza motility that has neither been addressed with molecular spiders nor in the experimental studies of influenza motility, is the relation between receptor specificity and directionality of the virus. We briefly mentioned that the human mucus is rich in 2,3-SLN, whereas the epithelial cells that are most commonly infected by influenza are richer in 2,6-SLN. We hypothesize that the balance between cleaving and binding in human influenza for 2,3-SLN is different from 2,6-SLN. Moderate binding to 2,3SLN combined with fast cleaving would result in strongly superdiffusive motion with a high chance of dissociation. This dissociation is, however, strongly inhibited by the 3D structure of the mucus. At the cell surface, where dissociation is more disadvantageous, a stronger binding to 2,6-SLN combined with moderate cleaving still results in superdiffusive motion to find a suitable spot for endocytosis, but with lower chance of dissociation. At the interface between the mucus and the cell, the binding to $2,6-\mathrm{SLN}$ on the cell would then be strongly favored over 2,3-SLN on the mucus.

\subsection{Conclusions}

We have argued that the multivalent display of NA and HA on the surface of influenza makes the virus a receptor-cleaving molecular walker. In its molecular walking, the virus resembles the synthetic molecular spiders, but the virus may 
achieve additional directionality by the asymmetric organization of HA and NA on its surface. It is likely that the surface-bound motility of influenza plays a key role in the transmission and propagation of the virus.

We expect that studies of artificial receptor-cleaving molecular walkers on heterogeneous surfaces can further our understanding of influenza as viruses that actively find access to their host cells, can help to explain and predict the evolutionary changes of influenza viruses, and can help identify possible new targets for antivirals in the transmission cycle of influenza.

\subsection{Acknowledgements}

Erik Hamming is thanked for his part in conceiving, performing, and writing this study. Erhard van der Vries (UMC Utrecht), Erik de Vries and Cornelis A. M. de Haan (University Utrecht) are acknowledged for helpful discussions.

\subsection{References}

1. de Graaf, M. \& Fouchier, R. A. M. Role of receptor binding specificity in influenza A virus transmission and pathogenesis. EMBO J. 33, 823-841 (2014).

2. Byrd-Leotis, L., Cummings, R. D. \& Steinhauer, D. A. The Interplay between the Host Receptor and Influenza Virus Hemagglutinin and Neuraminidase. Int. J. Mol. Sci. 18, 1541 (2017).

3. Neumann, G. \& Kawaoka, Y. Transmission of influenza A viruses. Virology 479-480, 234246 (2015).

4. Xu, R., Zhu, X., McBride, R., Nycholat, C. M., Yu, W., Paulson, J. C. \& Wilson, I. A. Functional Balance of the Hemagglutinin and Neuraminidase Activities Accompanies the Emergence of the 2009 H1N1 Influenza Pandemic. J. Virol. 86, 9221-9232 (2012).

5. Benton, D. J., Martin, S. R., Wharton, S. A. \& McCauley, J. W. Biophysical measurement of the balance of influenza A hemagglutinin and neuraminidase activities. J. Biol. Chem. 290, 6516-6521 (2015).

6. Gambaryan, A. S. \& Matrosovich, M. N. What adaptive changes in hemagglutinin and neuraminidase are necessary for emergence of pandemic influenza virus from its avian precursor? Biochem. 80, 872-880 (2015).

7. Wagner, R., Matrosovich, M. \& Klenk, H.-D. Functional balance between haemagglutinin and neuraminidase in influenza virus infections. Rev. Med. Virol. 12, 159-166 (2002).

8. Behera, A. K., Basu, S. \& Cherian, S. S. Molecular mechanism of the enhanced viral fitness contributed by secondary mutations in the hemagglutinin protein of oseltamivir resistant H1N1 influenza viruses: Modeling studies of antibody and receptor binding. Gene 557, 19-27 (2015).

9. Ward, M. J., Lycett, S. J., Avila, D., Bollback, J. P. \& Leigh Brown, A. J. Evolutionary interactions between haemagglutinin and neuraminidase in avian influenza. BMC Evol. 
Biol. 13, 222 (2013).

10. Diederich, S., Berhane, Y., Embury-Hyatt, C., Hisanaga, T., Handel, K., Cottam-Birt, C., Ranadheera, C., Kobasa, D. \& Pasick, J. Hemagglutinin-Neuraminidase Balance Influences the Virulence Phenotype of a Recombinant H5N3 Influenza A Virus Possessing a Polybasic HA 0 Cleavage Site. J. Virol. 89, 10724-10734 (2015).

11. Gulati, U., Wu, W., Gulati, S., Kumari, K., Waner, J. L. \& Air, G. M. Mismatched hemagglutinin and neuraminidase specificities in recent human $\mathrm{H} 3 \mathrm{~N} 2$ influenza viruses. Virology 339, 12-20 (2005).

12. Baigent, S. J. \& McCauley, J. W. Glycosylation of haemagglutinin and stalk-length of neuraminidase combine to regulate the growth of avian influenza viruses in tissue culture. Virus Res. 79, 177-185 (2001).

13. Gen, F., Yamada, S., Kato, K., Akashi, H., Kawaoka, Y. \& Horimoto, T. Attenuation of an influenza $A$ virus due to alteration of its hemagglutinin-neuraminidase functional balance in mice. Arch. Virol. 158, 1003-1011 (2013).

14. Chen, Q., Huang, S., Chen, J., Zhang, S. \& Chen, Z. NA Proteins of Influenza A Viruses H1N1/2009, H5N1, and H9N2 Show Differential Effects on Infection Initiation, Virus Release, and Cell-Cell Fusion. PLoS One 8, e54334 (2013).

15. Sakai, T., Nishimura, S. I., Naito, T. \& Saito, M. Influenza A virus hemagglutinin and neuraminidase act as novel motile machinery. Sci. Rep. 7, 45043 (2017).

16. Guo, H., Rabouw, H., Slomp, A., Dai, M., van der Vegt, F., van Lent, J. W. M., McBride, R., Paulson, J. C., de Groot, R. J., van Kuppeveld, F. J. M., de Vries, E. \& de Haan, C. A. M. Kinetic analysis of the influenza A virus HA/NA balance reveals contribution of NA to virus-receptor binding and NA-dependent rolling on receptor-containing surfaces. PLOS Pathog. 14, e1007233 (2018).

17. Müller, M., Lauster, D., Wildenauer, H. H. K., Herrmann, A. \& Block, S. Mobility-Based Quantification of Multivalent Virus-Receptor Interactions: New Insights Into Influenza A Virus Binding Mode. Nano Lett. 19, 1875-1882 (2019).

18. Vahey, M. D. \& Fletcher, D. A. Influenza A virus surface proteins are organized to help penetrate host mucus. elife 8, 1-24 (2019).

19. Block, S. M. Kinesin Motor Mechanics: Binding, Stepping, Tracking, Gating, and Limping. Biophys. J. 92, 2986-2995 (2007).

20. Hancock, W. O. Bidirectional cargo transport: moving beyond tug of war. Nat. Rev. Mol. Cell Biol. 15, 615-628 (2014).

21. Hammer, J. A. \& Sellers, J. R. Walking to work: Roles for class $v$ myosins as cargo transporters. Nat. Rev. Mol. Cell Biol. 13, 13-26 (2012).

22. Clemmens, J., Hess, H., Lipscomb, R., Hanein, Y., Böhringer, K. F., Matzke, C. M., Bachand, G. D., Bunker, B. C. \& Vogel, V. Mechanisms of Microtubule Guiding on Microfabricated Kinesin-Coated Surfaces: Chemical and Topographic Surface Patterns. Langmuir 19, 10967-10974 (2003).

23. Sherman, W. B. \& Seeman, N. C. A precisely controlled DNA biped walking device. Nano Lett. 4, 1203-1207 (2004).

24. Leigh, D. A., Lewandowska, U., Lewandowski, B. \& Wilson, M. R. in Multistage Mol. Methods Appl. Chem. 111-138 (2014). doi:10.1007/128_2014_546

25. Perl, A., Gomez-Casado, A., Thompson, D., Dam, H. H., Jonkheijm, P., Reinhoudt, D. N. \& 


\section{Chapter 7}

Huskens, J. Gradient-driven motion of multivalent ligand molecules along a surface functionalized with multiple receptors. Nat. Chem. 3, 317-322 (2011).

26. Fredy, J. W., Méndez-Ardoy, A., Kwangmettatam, S., Bochicchio, D., Matt, B., Stuart, M. C. A., Huskens, J., Katsonis, N., Pavan, G. M. \& Kudernac, T. Molecular photoswitches mediating the strain-driven disassembly of supramolecular tubules. Proc. Natl. Acad. Sci. U.S.A. 114, 11850-11855 (2017).

27. Kelly, T. R. Molecular Motors: Synthetic DNA-Based Walkers Inspired by Kinesin. Angew. Chem. Int. Ed. 44, 4124-4127 (2005).

28. Zhang, Z., Goldtzvik, Y. \& Thirumalai, D. Parsing the roles of neck-linker docking and tethered head diffusion in the stepping dynamics of kinesin. Proc. Natl. Acad. Sci. U.S.A. 114, E9838-E9845 (2017).

29. von Delius, M. \& Leigh, D. A. Walking molecules. Chem. Soc. Rev. 40, 3656 (2011).

30. Yin, P., Yan, H., Daniell, X. G., Turberfield, A. J. \& Reif, J. H. A Unidirectional DNA Walker That Moves Autonomously along a Track. Angew. Chem. Int. Ed. 43, 4906-4911 (2004).

31. Martin, C. J., Lee, A. T. L., Adams, R. W. \& Leigh, D. A. Enzyme-Mediated Directional Transport of a Small-Molecule Walker with Chemically Identical Feet. J. Am. Chem. Soc. 139, 11998-112002 (2017).

32. Pei, R., Taylor, S. K., Stefanovic, D., Rudchenko, S., Mitchell, T. E. \& Stojanovic, M. N. Behavior of polycatalytic assemblies in a substrate-displaying matrix. J. Am. Chem. Soc. 128, 12693-12699 (2006).

33. Chlanda, P. \& Zimmerberg, J. Protein-lipid interactions critical to replication of the influenza A virus. FEBS Lett. 590, 1940-1954 (2016).

34. Sun, X. \& Whittaker, G. R. in Adv. Exp. Med. Biol. 790, 72-82 (2006).

35. Lund, K., Manzo, A. J., Dabby, N., Michelotti, N., Johnson-Buck, A., Nangreave, J., Taylor, S., Pei, R., Stojanovic, M. N., Walter, N. G., Winfree, E. \& Yan, H. Molecular robots guided by prescriptive landscapes. Nature 465, 206-209 (2010).

36. Semenov, O., Olah, M. J. \& Stefanovic, D. Mechanism of diffusive transport in molecular spider models. Phys. Rev. E 83, 021117 (2011).

37. Tian, Y., He, Y., Chen, Y., Yin, P. \& Mao, C. A DNAzyme That Walks Processively and Autonomously along a One-Dimensional Track. Angew. Chem. Int. Ed. 44, 4355-4358 (2005).

38. Samii, L., Linke, H., Zuckermann, M. J. \& Forde, N. R. Biased motion and molecular motor properties of bipedal spiders. Phys. Rev. E - Stat. Nonlinear, Soft Matter Phys. 81, 1-11 (2010).

39. Olah, M. J. \& Stefanovic, D. in Lect. Notes Comput. Sci. (including Subser. Lect. Notes Artif. Intell. Lect. Notes Bioinformatics) 6937 LNCS, 160-174 (2011).

40. Korosec, C. S., Zuckermann, M. J. \& Forde, N. R. Dimensionality-dependent crossover in motility of polyvalent burnt-bridges ratchets. Phys. Rev. E 98, 1-11 (2018).

41. Stefanovic, D. A simplified account of cooperative effects in molecular walker teams. in Proc. 5th ACM Int. Conf. Nanoscale Comput. Commun. - NANOCOM'18 1-2 (ACM Press, 2018). doi:10.1145/3233188.3233218

42. Samii, L., Blab, G. A., Bromley, E. H. C., Linke, H., Curmi, P. M. G., Zuckermann, M. J. \& Forde, N. R. Time-dependent motor properties of multipedal molecular spiders. Phys. Rev. E - Stat. Nonlinear, Soft Matter Phys. 84, 1-11 (2011). 
43. Antal, T. \& Krapivsky, P. L. Molecular spiders with memory. Phys. Rev. E-Stat. Nonlinear, Soft Matter Phys. 76, 1-9 (2007).

44. Xiong, X., Coombs, P. J., Martin, S. R., Liu, J., Xiao, H., McCauley, J. W., Locher, K., Walker, P. A., Collins, P. J., Kawaoka, Y., Skehel, J. J. \& Gamblin, S. J. Receptor binding by a ferrettransmissible H5 avian influenza virus. Nature 497, 392-396 (2013).

45. Rameix-Welti, M. A., Munier, S., Le Gal, S., Cuvelier, F., Agou, F., Enouf, V., Naffakh, N. \& Van Der Werf, S. Neuraminidase of 2007-2008 influenza A(H1N1) viruses shows increased affinity for sialic acids due to the D344N substitution. Antivir. Ther. 16, 597603 (2011).

46. Zhu, X., McBride, R., Nycholat, C. M., Yu, W., Paulson, J. C. \& Wilson, I. A. Influenza Virus Neuraminidases with Reduced Enzymatic Activity That Avidly Bind Sialic Acid Receptors. J. Virol. 86, 13371-13383 (2012).

47. Blixt, O., Head, S., Mondala, T., Scanlan, C., Huflejt, M. E., Alvarez, R., Bryan, M. C., Fazio, F., Calarese, D., Stevens, J., Razi, N., Stevens, D. J., Skehel, J. J., van Die, I., Burton, D. R., Wilson, I. a, Cummings, R., Bovin, N., Wong, C.-H. \& Paulson, J. C. Printed covalent glycan array for ligand profiling of diverse glycan binding proteins. Proc. Natl. Acad. Sci. U.S.A. 101, 17033-17038 (2004).

48. Stevens, J., Blixt, O., Glaser, L., Taubenberger, J. K., Palese, P., Paulson, J. C. \& Wilson, I. A. Glycan microarray analysis of the hemagglutinins from modern and pandemic influenza viruses reveals different receptor specificities. J. Mol. Biol. 355, 1143-1155 (2006).

49. Harris, A., Cardone, G., Winkler, D. C., Heymann, J. B., Brecher, M., White, J. M. \& Steven, A. C. Influenza virus pleiomorphy characterized by cryoelectron tomography. Proc. Natl. Acad. Sci. U.S.A. 103, 19123-19127 (2006).

50. Chlanda, P., Schraidt, O., Kummer, S., Riches, J., Oberwinkler, H., Prinz, S., Kräusslich, H.G. \& Briggs, J. A. G. Structural Analysis of the Roles of Influenza A Virus MembraneAssociated Proteins in Assembly and Morphology. J. Virol. 89, 8957-8966 (2015).

51. Ohkura, T., Momose, F., Ichikawa, R., Takeuchi, K. \& Morikawa, Y. Influenza A Virus Hemagglutinin and Neuraminidase Mutually Accelerate Their Apical Targeting through Clustering of Lipid Rafts. J. Virol. 88, 10039-10055 (2014).

52. Rossman, J. S. \& Lamb, R. A. Influenza virus assembly and budding. Virology 411, 229236 (2011).

53. Madsen, J. J., Grime, J. M. A., Rossman, J. S. \& Voth, G. A. Entropic forces drive clustering and spatial localization of influenza A M2 during viral budding. Proc. Natl. Acad. Sci. U.S.A. 115, E8595-E8603 (2018).

54. Dadonaite, B., Vijayakrishnan, S., Fodor, E., Bhella, D. \& Hutchinson, E. C. Filamentous influenza viruses. J. Gen. Virol. 97, 1755-1764 (2016).

55. Rust, M. J., Lakadamyali, M., Zhang, F. \& Zhuang, X. Assembly of endocytic machinery around individual influenza viruses during viral entry. Nat. Struct. Mol. Biol. 11, 567-573 (2004).

56. Martinez-Veracoechea, F. J. \& Frenkel, D. Designing super selectivity in multivalent nanoparticle binding. Proc. Natl. Acad. Sci. U.S.A. 108, 10963-10968 (2011).

57. Button, B., Cai, L.-H., Ehre, C., Kesimer, M., Hill, D. B., Sheehan, J. K., Boucher, R. C. \& Rubinstein, M. A Periciliary Brush Promotes the Lung Health by Separating the Mucus 


\section{Chapter 7}

Layer from Airway Epithelia. Science 337, 937-941 (2012).

58. Taherali, F., Varum, F. \& Basit, A. W. A slippery slope: On the origin, role and physiology of mucus. Adv. Drug Deliv. Rev. 124, 16-33 (2018).

59. Bansil, R. \& Turner, B. S. The biology of mucus: Composition, synthesis and organization. Adv. Drug Deliv. Rev. 124, 3-15 (2018).

60. Schuster, B. S., Suk, J. S., Woodworth, G. F. \& Hanes, J. Nanoparticle diffusion in respiratory mucus from humans without lung disease. Biomaterials 34, 3439-3446 (2013).

61. Cohen, M., Zhang, X.-Q., Senaati, H. P., Chen, H.-W., Varki, N. M., Schooley, R. T. \& Gagneux, P. Influenza A penetrates host mucus by cleaving sialic acids with neuraminidase. Virol. J. 10, 321 (2013).

62. Yang, X., Steukers, L., Forier, K., Xiong, R., Braeckmans, K., Van Reeth, K. \& Nauwynck, H. A Beneficiary Role for Neuraminidase in Influenza Virus Penetration through the Respiratory Mucus. PLoS One 9, e110026 (2014).

63. Gagneux, P., Cheriyan, M., Hurtado-Ziola, N., van der Linden, E. C. M. B., Anderson, D., McClure, H., Varki, A. \& Varki, N. M. Human-specific Regulation of a2-6-linked Sialic Acids. J. Biol. Chem. 278, 48245-48250 (2003).

64. Couceiro, J. N. S. S., Paulson, J. C. \& Baum, L. G. Influenza virus strains selectively recognize sialyloligosaccharides on human respiratory epithelium; the role of the host cell in selection of hemagglutinin receptor specificity. Virus Res. 29, 155-165 (1993).

65. Zanin, M., Marathe, B., Wong, S.-S., Yoon, S.-W., Collin, E., Oshansky, C., Jones, J., Hause, B. \& Webby, R. Pandemic Swine H1N1 Influenza Viruses with Almost Undetectable Neuraminidase Activity Are Not Transmitted via Aerosols in Ferrets and Are Inhibited by Human Mucus but Not Swine Mucus. J. Virol. 89, 5935-5948 (2015).

66. Zanin, M., Baviskar, P., Webster, R. \& Webby, R. The Interaction between Respiratory Pathogens and Mucus. Cell Host Microbe 19, 159-168 (2016).

67. Kesimer, M., Ehre, C., Burns, K. A., Davis, C. W., Sheehan, J. K. \& Pickles, R. J. Molecular organization of the mucins and glycocalyx underlying mucus transport over mucosal surfaces of the airways. Mucosal Immunol. 6, 379-392 (2013).

68. Lin, Y. P., Xiong, X., Wharton, S. A., Martin, S. R., Coombs, P. J., Vachieri, S. G., Christodoulou, E., Walker, P. A., Liu, J., Skehel, J. J., Gamblin, S. J., Hay, A. J., Daniels, R. S. \& McCauley, J. W. Evolution of the receptor binding properties of the influenza A(H3N2) hemagglutinin. Proc. Natl. Acad. Sci. U.S.A. 109, 21474-21479 (2012).

69. Yehl, K., Mugler, A., Vivek, S., Liu, Y., Zhang, Y., Fan, M., Weeks, E. R. \& Salaita, K. Highspeed DNA-based rolling motors powered by RNase H. Nat. Nanotechnol. 11, 184-190 (2016).

70. Blanchard, A. T., Bazrafshan, A. S., Yi, J., Eisman, J. T., Yehl, K. M., Bian, T., Mugler, A. \& Salaita, K. Highly Polyvalent DNA Motors Generate $100+\mathrm{pN}$ of Force via Autochemophoresis. Nano Lett. 19, 6977-6986 (2019). 
Influenza as a molecular walker 
Chapter 8

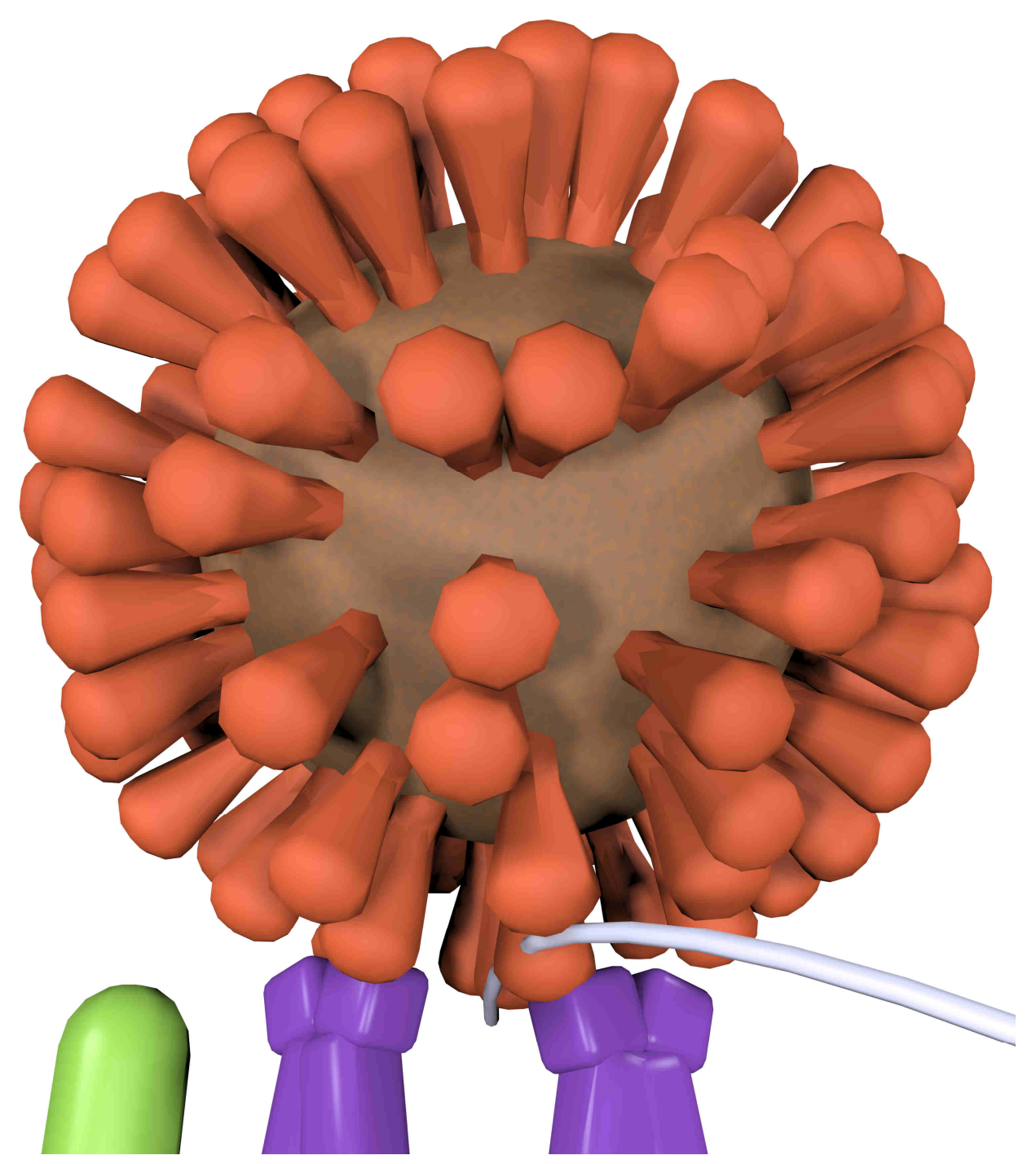




\section{Future perspectives}

This chapter provides an outlook beyond the research described in the previous chapters. The implications of the work are discussed further as well as possible applications, without attempting to be exhaustive.

The research discussed in this thesis offers new insights into the binding of influenza A viruses and has introduced a new method to assess virus binding. Multivalent affinity profiling (MAP) is a versatile tool that can assess the binding of viruses, even under shear flow (Chapter 5) and with active NA (Chapter 6). Potentially, MAP could be used not only for any type of influenza virus but also for other viruses or any multivalent particle, or to evaluate binding inhibitors. However, in this thesis only the influenza virus PR8 was used. The broader applicability remains therefore yet unproven so that further application of MAP should be accompanied by further validation of the method and the platform. In particular, the relationship between sample preparation and the resulting receptor densities requires external calibration for MAP as well as for the other state-of-the-art techniques - quartz crystal microbalance (QCM) and biolayer interferometry (BLI) - that were used in Chapter 4. This would make the use threshold receptor densities generalizable regardless of the technique by which they are acquired. Short-term extensions lie in the directions of: testing different influenza strains and correlating binding to infectivity, testing other virus-receptor combinations (for example, for SARS-CoV-2), testing different nanoparticles (for example, artificially made HA constructs), and particle tracking experiments to gain a better mechanistic understanding of virus dynamics. 


\subsection{MAP as a biosensor}

In this thesis, the length and density of surface-bound receptors proved to direct virus binding (Chapter 4). This behavior illustrates the importance of considering the effects of receptor display and surface properties on receptorligand interactions for biosensors in general. The decoupling of the receptor and virus binding from the assembly of the supported lipid bilayers (Chapter 3 ) proved a critical step in achieving the control that was needed to study virus binding as a function of receptor density.

Supported lipid bilayers (SLBs) have excellent antifouling properties and mimic the properties of biological membranes better than most surface coatings. ${ }^{1,2}$ However, their dynamicity and self-healing properties limit their long-term stability in the absence of a continuous replenishment of lipids that are lost from the SLB. ${ }^{3-5}$ Therefore, additional steps may be necessary in the preparation of SLBs in case long-term stability is desired. ${ }^{2}$ This includes the potential reuse of the receptor density gradients in the MAP chip. Influenza viruses are fully released from glycan receptors at $\mathrm{pH} 2 .{ }^{6}$ The receptor density gradients in the MAP chip may therefore be regenerated with an acidic solution, provided that stability of the SLBs is ensured. When combined with automated solution change and imaging, the MAP platform may be fit for high-throughput screening of receptor specificity of influenza viruses or the inhibitory potential of antiviral drugs.

\subsection{Interaction of influenza with the glycocalyx}

The study of PR8 binding to different receptors has shown that the virus needs a receptor density that is high enough to form sufficient numbers of interactions (Chapter 4). The number of interactions that is needed depends on the equilibrium constant of the HA-receptor complex. Because longer glycans can access a larger contact area of the virus, glycan length and density are partly interchangeable. Additionally, longer glycans can more easily conform to the orientation of the receptor binding domains (RBDs) on the virus so that the cost of the loss of conformational entropy of forming an interaction is lower than for shorter glycans. Interestingly, short 2,6-linked sialoglycans are more restricted from accessing the RBDs than short 2,3-linked ones, but long 2,6-linked ones are not. Additionally, two long 2,6-linked glycans on the same streptavidin are 
more likely to interact with two RBDs on the same HA than 2,3-linked glycans. On a glycan array, the binding to a long linear 2,6-linked glycan was relatively low, both in comparison to a similar 2,3-linked glycan and to branched 2,6linked glycans. This would mean that a preference for 2,6-linked glycans automatically implies a preference for longer branched glycans. A stronger or weaker preference for branched glycans may be encoded in the HA headgroup, which can have attractive or repulsive interactions with the glycan chains.

To apply the implications of these findings to predict cell and tissue tropism and host species specificity from receptor specificity, a better understanding of the composition of the glycocalyx is necessary. Common methods to study the glycan composition of the glycocalyx include lectin staining, ${ }^{7-9}$ which is only semi-quantitative, and mass spectrometry, ${ }^{10,11}$ which provides information about the diversity of glycans but is not quantitative. A quantitative assessment of the density of functional receptors for the influenza virus could be achieved by controlled manipulation of the sialic acid composition of the glycocalyx in tissue cultures using sialidases and sialyltransferases, and by comparing the virus binding on the manipulated tissue with the threshold receptor densities in MAP. Alternatively, sialic acid may be released from functional and accessible receptors on cells using a virus with high NA activity. The released sialic acid can be separated from the viruses and cells by centrifugation and subsequently assayed in the supernatant to determine their density on the cells from which they were released. ${ }^{12}$

Combined with more quantitative information about the glycocalyx of different host species, MAP may be used to identify in more detail which receptor binding properties are selected for by influenza viruses in the adaptation to different hosts. Of particular interest would be the difference between spherical and more elongated viruses, and the role of polydispersity in superselective virus binding. This knowledge would be helpful in understanding zoonoses and in assaying the potential of emerging influenza strains to cause epidemic or epizootic infections in a new host.

By comparing the availability of different functional receptors in different parts of the human airways, the receptor specificity of influenza viruses may be related to transmissibility and pathogenicity. ${ }^{13,14}$ It would also be interesting to compare the effect of shear flow on the binding of different influenza viruses to 
different receptors, because this might have a role in their airborne transmissibility if variations in airflow lead to different release rates of different viruses.

Because influenza viruses have a broad distribution of shapes and sizes that varies between strains, ${ }^{15}$ the effect of these variations on receptor binding would also be an interesting subject of study. The theory in Chapter 4 suggests that larger viruses, which can form more interactions at the same receptor density, have a lower threshold receptor density. In a polydisperse virus sample, the threshold receptor density would be determined by the largest viruses.

\subsection{Antiviral drugs}

Understanding the multivalent binding of viruses plays a central role in the development of new classes of antiviral drugs. ${ }^{16-18}$ Multivalent particles, polymers, or nanomaterial sheets are designed to bind viruses with high avidity and block their receptor binding. ${ }^{19-23}$ Some of these multivalent antivirals destroy the viruses to which they bind instead of relying on inhibition of the receptor binding alone. ${ }^{24-26}$ Regardless of whether the receptors on the antiviral are artificial or mimics of cellular receptors, the density of these receptors on the antiviral should be higher than the corresponding threshold receptor density in order to provide efficient multivalent binding, but further increasing their density may reduce the effectivity of the antiviral due to steric repulsion. ${ }^{27}$ The MAP chip can not only help in the design of these drugs but also in assessing their inhibitory potential when viruses mixed with the antiviral bind less efficiently to the receptors on the surface.

\subsection{Application to other pathogens}

Because most if not all animal viruses are multivalent, ${ }^{28}$ MAP can be used to assess their binding and help in the development of antivirals for other viruses than influenza as well. Other viruses that bind to glycan receptors include polyomavirus, human rotavirus, rubella, mumps virus, herpes simplex virus, Newcastle disease virus, coronavirus OC43, vesicular stomatitis virus, rabies virus, simian virus 40 , and infectious bronchitis virus. ${ }^{28-32}$ Instead of glycans, proteins, protein fragments or biopolymers may be used as receptors. While no receptor type is excluded a priori, controlled immobilization of these larger and 
more complex receptors in a gradient may require additional steps. A requirement for using MAP is that the virus binds superselectively, which may not be the case at meaningful concentrations for all viruses because the binding to protein receptors may be much stronger than to glycans. ${ }^{33}$

In addition to viruses, MAP may also be used for bacteria as many bacteria bind multivalently to glycan receptors as well. ${ }^{28}$ However, due to their size, the diffusion coefficient and typical concentration of bacteria are lower than those of viruses, and therefore it may be more difficult to bind sufficient numbers of bacteria on the surface of the MAP chip for an accurate reading. ${ }^{34}$

\subsubsection{SARS-CoV-2}

The virus that is of most urgent interest is SARS-CoV2, the causative agent of COVID-19. Like the Influenza A virus, SARS-CoV2 is an enveloped virus of approximately $100 \mathrm{~nm} .{ }^{35}$ The surface of SARS-CoV2 is decorated with spike (S) proteins that bind to the protein receptor ACE2. ${ }^{36}$ Unlike influenza $A$ and $B$ viruses and coronavirus OC43, SARS-CoV2 does not possess a second, receptorcleaving surface protein. ${ }^{37-39}$ The polysaccharide heparan sulfate acts as a first attachment point for the $\mathrm{S}$ protein and allosterically promotes the binding to ACE2. ${ }^{40}$ The cell surface serine protease TMPRSS2 is used by the virus to prime its $S$ protein for cell entry. ${ }^{36}$ Like MERS-CoV, ${ }^{41}$ the $S$ protein of SARS-CoV- 2 can also bind sialic acid. ${ }^{42}$ It is suspected that the multivalent binding to sialic acid helps coronaviruses in the binding and plays a role in host cell recognition. MAP could be used to assess the multivalent binding of SARS-CoV2 to these different receptors in a density-controlled fashion to better understand their respective roles in virus tropism.

To efficiently study the density-dependent binding to receptors that may allosterically affect each other, two-dimensional gradients may be used in which the density of two receptors is varied in orthogonal directions. The fabrication of such gradients may be achieved using a chip design where the electrodes run parallel to the microchannel. A streptavidin gradient could then be formed at elevated temperature by shear force instead of electrophoresis ${ }^{43}$ and the electrodes could be used to form an orthogonal surface gradient using electrochemically activated chemistry. ${ }^{44}$ 


\subsection{Cancer therapies}

Apart from the study of pathogen binding, MAP may be used to evaluate and develop targeted drug delivery carriers. The delivery of chemotherapy via nanocontainers that bind selectively to tumor cells can improve the efficacy of cancer treatments. ${ }^{45-47}$ Often the targeted receptors on tumor cells are not unique but are overexpressed..$^{48-51}$ This means that the targeting can benefit from superselective binding. ${ }^{52-55}$ MAP may be used to guide the design of superselective drug carriers with a threshold receptor density that distinguishes healthy from diseased cells. The receptor-cleaving activity of the influenza virus that enhances its superselectivity (Chapter 6) may also inspire new targeting strategies.

\subsection{Virus motility}

The active motility that influenza viruses achieve by cleaving their receptors (Chapter 7) is also of great significance because it suggests that viruses may play a much more active role in their own lifecycle than is generally ascribed to them. ${ }^{56,57}$ Active motility as part of the reproduction cycle is generally considered a property of living organisms, to which viruses are not counted. ${ }^{58-}$ ${ }^{60}$ Because more viruses have receptor-cleaving functions, ${ }^{61}$ this classification may need to be reconsidered.

In the future, influenza-like motility may also find applications. Nanorobots that travel through the body and perform repairs where needed have long been an improbable dream. ${ }^{62} \mathrm{~A}$ better understanding of the motility of influenza viruses and the structure of the glycocalyces on cells may bring this dream within reach.

\subsection{References}

1. Di lorio, D. \& Huskens, J. Surface Modification with Control over Ligand Density for the Study of Multivalent Biological Systems. ChemistryOpen 9, 53-66 (2020).

2. Van Weerd, J., Karperien, M. \& Jonkheijm, P. Supported Lipid Bilayers for the Generation of Dynamic Cell-Material Interfaces. Adv. Healthc. Mater. 4, 2743-2779 (2015).

3. Hardy, G. J., Nayak, R. \& Zauscher, S. Model cell membranes: Techniques to form complex biomimetic supported lipid bilayers via vesicle fusion. Curr. Opin. Colloid Interface Sci. 18, 448-458 (2013).

4. Lind, T. K., Cárdenas, M. \& Wacklin, H. P. Formation of supported lipid bilayers by vesicle fusion: effect of deposition temperature. Langmuir 30, 7259-63 (2014). 
5. Richter, R. P., Bérat, R. \& Brisson, A. R. Formation of Solid-Supported Lipid Bilayers: An Integrated View. Langmuir 22, 3497-3505 (2006).

6. Guo, H., Rabouw, H., Slomp, A., Dai, M., van der Vegt, F., van Lent, J. W. M., McBride, R., Paulson, J. C., de Groot, R. J., van Kuppeveld, F. J. M., de Vries, E. \& de Haan, C. A. M. Kinetic analysis of the influenza A virus $\mathrm{HA} / \mathrm{NA}$ balance reveals contribution of NA to virus-receptor binding and NA-dependent rolling on receptor-containing surfaces. PLoS Pathog. 14, e1007233 (2018).

7. van Riel, D., den Bakker, M. A., Leijten, L. M. E., Chutinimitkul, S., Munster, V. J., de Wit, E., Rimmelzwaan, G. F., Fouchier, R. A. M., Osterhaus, A. D. M. E. \& Kuiken, T. Seasonal and pandemic human influenza viruses attach better to human upper respiratory tract epithelium than avian influenza viruses. Am. J. Pathol. 176, 1614-8 (2010).

8. Gagneux, P., Cheriyan, M., Hurtado-Ziola, N., van der Linden, E. C. M. B., Anderson, D., McClure, H., Varki, A. \& Varki, N. M. Human-specific Regulation of a2-6-linked Sialic Acids. J. Biol. Chem. 278, 48245-48250 (2003).

9. Byrd-Leotis, L., Cummings, R. D. \& Steinhauer, D. A. The Interplay between the Host Receptor and Influenza Virus Hemagglutinin and Neuraminidase. Int. J. Mol. Sci. 18, 1541 (2017).

10. Jia, N., Byrd-Leotis, L., Matsumoto, Y., Gao, C., Wein, A. N., Lobby, J. L., Kohlmeier, J. E., Steinhauer, D. A. \& Cummings, R. D. The Human Lung Glycome Reveals Novel Glycan Ligands for Influenza A Virus. Sci. Rep. 10, 5320 (2020).

11. Byrd-Leotis, L., Jia, N., Dutta, S., Trost, J. F., Gao, C., Cummings, S. F., Braulke, T., MüllerLoennies, S., Heimburg-Molinaro, J., Steinhauer, D. A. \& Cummings, R. D. Influenza binds phosphorylated glycans from human lung. Sci. Adv. 5, 1-10 (2019).

12. Seaman, G., Knox, R., Nordt, F. \& Regan, D. Red cell agins. I. Surface charge density and sialic acid content of density-fractionated human erythrocytes. Blood 50, 1001-1011 (1977).

13. Richard, M., van den Brand, J. M. A., Bestebroer, T. M., Lexmond, P., de Meulder, D., Fouchier, R. A. M., Lowen, A. C. \& Herfst, S. Influenza A viruses are transmitted via the air from the nasal respiratory epithelium of ferrets. Nat. Commun. 11, 766 (2020).

14. Van Riel, D., Munster, V. J., de Wit, E., Rimmelzwaan, G. F., Fouchier, R. A. M., Osterhaus, A. D. M. E. \& Kuiken, T. H5N1 Virus Attachment to Lower Respiratory Tract. Science 312, 399-399 (2006).

15. Vahey, M. D. \& Fletcher, D. A. Low-Fidelity Assembly of Influenza A Virus Promotes Escape from Host Cells. Cell 176, 281-294 (2019).

16. Heida, R., Bhide, Y. C., Gasbarri, M., Kocabiyik, Ö., Stellacci, F., Huckriede, A. L. W., Hinrichs, W. L. J. \& Frijlink, H. W. Advances in the development of entry inhibitors for sialic-acidtargeting viruses. Drug Discov. Today 102448 (2020). doi:10.1016/j.drudis.2020.10.009

17. Kwon, P. S., Ren, S., Kwon, S.-J., Kizer, M. E., Kuo, L., Xie, M., Zhu, D., Zhou, F., Zhang, F., Kim, D., Fraser, K., Kramer, L. D., Seeman, N. C., Dordick, J. S., Linhardt, R. J., Chao, J. \& Wang, X. Designer DNA architecture offers precise and multivalent spatial patternrecognition for viral sensing and inhibition. Nat. Chem. 12, 26-35 (2020). 


\section{Chapter 8}

18. Lauster, D., Klenk, S., Ludwig, K., Nojoumi, S., Behren, S., Adam, L., Stadtmüller, M., Saenger, S., Zimmler, S., Hönzke, K., Yao, L., Hoffmann, U., Bardua, M., Hamann, A., Witzenrath, M., Sander, L. E., Wolff, T., Hocke, A. C., Hippenstiel, S., De Carlo, S., Neudecker, J., Osterrieder, K., Budisa, N., Netz, R. R., Böttcher, C., Liese, S., Herrmann, A. \& Hackenberger, C. P. R. Phage capsid nanoparticles with defined ligand arrangement block influenza virus entry. Nat. Nanotechnol. 15, 373-379 (2020).

19. Nie, C., Parshad, B., Bhatia, S., Cheng, C., Stadtmüller, M., Oehrl, A., Kerkhoff, Y., Wolff, T. \& Haag, R. Topology-Matching Design of an Influenza-Neutralizing Spiky NanoparticleBased Inhibitor with a Dual Mode of Action. Angew. Chemie 132, 15662-15666 (2020).

20. Wallert, M., Nie, C., Anilkumar, P., Abbina, S., Bhatia, S., Ludwig, K., Kizhakkedathu, J. N., Haag, R. \& Block, S. Mucin-Inspired, High Molecular Weight Virus Binding Inhibitors Show Biphasic Binding Behavior to Influenza A Viruses. Small 16, 2004635 (2020).

21. Bhatia, S., Camacho, L. C. \& Haag, R. Pathogen Inhibition by Multivalent Ligand Architectures. J. Am. Chem. Soc. 138, 8654-8666 (2016).

22. Cohen, M., Senaati, H. P., Fisher, C. J., Huang, M. L., Gagneux, P. \& Godula, K. Synthetic mucus nanobarriers for identification of glycan-dependent primary influenza a infection inhibitors. ACS Cent. Sci. 2, 710-714 (2016).

23. Bhatia, S., Hilsch, M., Cuellar-Camacho, J. L., Ludwig, K., Nie, C., Parshad, B., Wallert, M., Block, S., Lauster, D., Böttcher, C., Herrmann, A. \& Haag, R. Adaptive Flexible Sialylated Nanogels as Highly Potent Influenza A Virus Inhibitors. Angew. Chem. Int. Ed. 59, 1241712422 (2020).

24. Jones, S. T., Cagno, V., Janeček, M., Ortiz, D., Gasilova, N., Piret, J., Gasbarri, M., Constant, D. A., Han, Y., Vuković, L., Král, P., Kaiser, L., Huang, S., Constant, S., Kirkegaard, K., Boivin, G., Stellacci, F. \& Tapparel, C. Modified cyclodextrins as broad-spectrum antivirals. Sci. Adv. 6, eaax9318 (2020).

25. Cagno, V., Andreozzi, P., D’Alicarnasso, M., Jacob Silva, P., Mueller, M., Galloux, M., Le Goffic, R., Jones, S. T., Vallino, M., Hodek, J., Weber, J., Sen, S., Janeček, E.-R., Bekdemir, A., Sanavio, B., Martinelli, C., Donalisio, M., Rameix Welti, M.-A., Eleouet, J.-F., Han, Y., Kaiser, L., Vukovic, L., Tapparel, C., Král, P., Krol, S., Lembo, D. \& Stellacci, F. Broad-spectrum nontoxic antiviral nanoparticles with a virucidal inhibition mechanism. Nat. Mater. 17, 195203 (2018).

26. Zacheo, A., Hodek, J., Witt, D., Mangiatordi, G. F., Ong, Q. K., Kocabiyik, O., Depalo, N., Fanizza, E., Laquintana, V., Denora, N., Migoni, D., Barski, P., Stellacci, F., Weber, J. \& Krol, S. Multi-sulfonated ligands on gold nanoparticles as virucidal antiviral for Dengue virus. Sci. Rep. 10, 9052 (2020).

27. Bhatia, S., Lauster, D., Bardua, M., Ludwig, K., Angioletti-Uberti, S., Popp, N., Hoffmann, U., Paulus, F., Budt, M., Stadtmüller, M., Wolff, T., Hamann, A., Böttcher, C., Herrmann, A. \& Haag, R. Linear polysialoside outperforms dendritic analogs for inhibition of influenza virus infection in vitro and in vivo. Biomaterials 138, 22-34 (2017).

28. Mammen, M., Choi, S.-K. \& Whitesides, G. M. Polyvalent Interactions in Biological Systems: Implications for Design and Use of Multivalent Ligands and Inhibitors. Angew. Chem. Int. Ed. 37, 2754-2794 (1998). 
29. Szklarczyk, O. M., González-Segredo, N., Kukura, P., Oppenheim, A., Choquet, D., Sandoghdar, V., Helenius, A., Sbalzarini, I. F. \& Ewers, H. Receptor Concentration and Diffusivity Control Multivalent Binding of Sv40 to Membrane Bilayers. PLoS Comput. Biol. 9, e1003310 (2013).

30. Forgione, R. E., Di Carluccio, C., Kubota, M., Manabe, Y., Fukase, K., Molinaro, A., Hashiguchi, T., Marchetti, R. \& Silipo, A. Structural basis for Glycan-receptor binding by mumps virus hemagglutinin-neuraminidase. Sci. Rep. 10, 1589 (2020).

31. Winter, C., Schwegmann-Weßels, C., Cavanagh, D., Neumann, U. \& Herrler, G. Sialic acid is a receptor determinant for infection of cells by avian Infectious bronchitis virus. J. Gen. Virol. 87, 1209-1216 (2006).

32. Peerboom, N., Block, S., Altgärde, N., Wahlsten, O., Möller, S., Schnabelrauch, M., Trybala, E., Bergström, T. \& Bally, M. Binding Kinetics and Lateral Mobility of HSV-1 on End-Grafted Sulfated Glycosaminoglycans. Biophys. J. 113, 1223-1234 (2017).

33. Walls, A. C., Park, Y.-J., Tortorici, M. A., Wall, A., McGuire, A. T. \& Veesler, D. Structure, Function, and Antigenicity of the SARS-CoV-2 Spike Glycoprotein. Cell 181, 281-292 (2020).

34. Van Weerd, J., Sankaran, S., Roling, O., Sukas, S., Krabbenborg, S., Huskens, J., le Gac, S., Ravoo, B. J., Karperien, M. \& Jonkheijm, P. A Microfluidic Device with Continuous Ligand Gradients in Supported Lipid Bilayers to Probe Effects of Ligand Surface Density and Solution Shear Stress on Pathogen Adhesion. Adv. Mater. Interfaces 3, 1600055 (2016).

35. Astuti, I. \& Ysrafil. Severe Acute Respiratory Syndrome Coronavirus 2 (SARS-CoV-2): An overview of viral structure and host response. Diabetes Metab. Syndr. Clin. Res. Rev. 14, 407-412 (2020).

36. Hoffmann, M., Kleine-Weber, H., Schroeder, S., Krüger, N., Herrler, T., Erichsen, S., Schiergens, T. S., Herrler, G., Wu, N.-H., Nitsche, A., Müller, M. A., Drosten, C. \& Pöhlmann, S. SARS-CoV-2 Cell Entry Depends on ACE2 and TMPRSS2 and Is Blocked by a Clinically Proven Protease Inhibitor. Cell 181, 271-280 (2020).

37. Ross, J., Maier, H. J. \& Walker, J. M. Coronaviruses. 1282, (Springer New York, 2015).

38. Bakkers, M. J. G., Lang, Y., Feitsma, L. J., Hulswit, R. J. G., de Poot, S. A. H., van Vliet, A. L. W., Margine, I., de Groot-Mijnes, J. D. F., van Kuppeveld, F. J. M., Langereis, M. A., Huizinga, E. G. \& de Groot, R. J. Betacoronavirus Adaptation to Humans Involved Progressive Loss of Hemagglutinin-Esterase Lectin Activity. Cell Host Microbe 21, 356-366 (2017).

39. Zeng, Q., Langereis, M. A., van Vliet, A. L. W., Huizinga, E. G. \& de Groot, R. J. Structure of coronavirus hemagglutinin-esterase offers insight into corona and influenza virus evolution. Proc. Natl. Acad. Sci. U.S.A. 105, 9065-9069 (2008).

40. Clausen, T. M., Sandoval, D. R., Spliid, C. B., Pihl, J., Perrett, H. R., Painter, C. D., Narayanan, A., Majowicz, S. A., Kwong, E. M., McVicar, R. N., Thacker, B. E., Glass, C. A., Yang, Z., Torres, J. L., Golden, G. J., Bartels, P. L., Porell, R. N., Garretson, A. F., Laubach, L., Feldman, J., Yin, X., Pu, Y., Hauser, B. M., Caradonna, T. M., Kellman, B. P., Martino, C., Gordts, P. L. S. M., Chanda, S. K., Schmidt, A. G., Godula, K., Leibel, S. L., Jose, J., Corbett, K. D., Ward, A. B., Carlin, A. F. \& Esko, J. D. SARS-CoV-2 Infection Depends on Cellular Heparan Sulfate and ACE2. Cell 183, 1043-1057 (2020). 


\section{Chapter 8}

41. Li, W., Hulswit, R. J. G., Widjaja, I., Raj, V. S., McBride, R., Peng, W., Widagdo, W., Tortorici, M. A., van Dieren, B., Lang, Y., van Lent, J. W. M., Paulson, J. C., de Haan, C. A. M., de Groot, R. J., van Kuppeveld, F. J. M., Haagmans, B. L. \& Bosch, B.-J. Identification of sialic acidbinding function for the Middle East respiratory syndrome coronavirus spike glycoprotein. Proc. Natl. Acad. Sci. U.S.A. 114, E8508-E8517 (2017).

42. Baker, A. N., Richards, S.-J., Guy, C. S., Congdon, T. R., Hasan, M., Zwetsloot, A. J., Gallo, A., Lewandowski, J. R., Stansfeld, P. J., Straube, A., Walker, M., Chessa, S., Pergolizzi, G., Dedola, S., Field, R. A. \& Gibson, M. I. The SARS-COV-2 Spike Protein Binds Sialic Acids and Enables Rapid Detection in a Lateral Flow Point of Care Diagnostic Device. ACS Cent. Sci. 6, 2046-2052 (2020).

43. Van Weerd, J., Krabbenborg, S. O., Eijkel, J., Karperien, M., Huskens, J. \& Jonkheijm, P. OnChip Electrophoresis in Supported Lipid Bilayer Membranes Achieved Using Low Potentials. J. Am. Chem. Soc. 136, 100-103 (2014).

44. Nicosia, C., Krabbenborg, S. O., Chen, P. \& Huskens, J. Shape-controlled fabrication of micron-scale surface chemical gradients via electrochemically activated copper(I) 'click' chemistry. J. Mater. Chem. B 1, 5417-5428 (2013).

45. Song, G., Cheng, L., Chao, Y., Yang, K. \& Liu, Z. Emerging Nanotechnology and Advanced Materials for Cancer Radiation Therapy. Adv. Mater. 29, 1-26 (2017).

46. Yu, G., Zhu, B., Shao, L., Zhou, J., Saha, M. L., Shi, B., Zhang, Z., Hong, T., Li, S., Chen, X. \& Stang, P. J. Host-guest complexation-mediated codelivery of anticancer drug and photosensitizer for cancer photochemotherapy. Proc. Natl. Acad. Sci. U.S.A. 116, 66186623 (2019).

47. Zhang, S., Chen, C., Xue, C., Chang, D., Xu, H., Salena, B. J., Li, Y. \& Wu, Z. Ribbon of DNA Lattice on Gold Nanoparticles for Selective Drug Delivery to Cancer Cells. Angew. Chem. Int. Ed. 59, 14584-14592 (2020).

48. Dalal, C., Saha, A. \& Jana, N. R. Nanoparticle Multivalency Directed Shifting of Cellular Uptake Mechanism. J. Phys. Chem. C 120, 6778-6786 (2016).

49. Yu, G., Zhao, X., Zhou, J., Mao, Z., Huang, X., Wang, Z., Hua, B., Liu, Y., Zhang, F., He, Z., Jacobson, O., Gao, C., Wang, W., Yu, C., Zhu, X., Huang, F. \& Chen, X. Supramolecular Polymer-Based Nanomedicine: High Therapeutic Performance and Negligible Long-Term Immunotoxicity. J. Am. Chem. Soc. 140, 8005-8019 (2018).

50. Aires, A., Cadenas, J. F., Guantes, R. \& Cortajarena, A. L. An experimental and computational framework for engineering multifunctional nanoparticles: Designing selective anticancer therapies. Nanoscale 9, 13760-13771 (2017).

51. Wang, J., Min, J., Eghtesadi, S. A., Kane, R. S. \& Chilkoti, A. Quantitative Study of the Interaction of Multivalent Ligand-Modified Nanoparticles with Breast Cancer Cells with Tunable Receptor Density. ACS Nano 14, 372-383 (2020).

52. Carlson, C. B., Mowery, P., Owen, R. M., Dykhuizen, E. C. \& Kiessling, L. L. Selective Tumor Cell Targeting Using Low-Affinity, Multivalent Interactions. ACS Chem. Biol. 2, 119-127 (2007). 
53. Scheepers, M. R. W., van IJzendoorn, L. J. \& Prins, M. W. J. Multivalent weak interactions enhance selectivity of interparticle binding. Proc. Natl. Acad. Sci. U.S.A. 117, 22690-22697 (2020).

54. Wang, S. \& Dormidontova, E. E. Selectivity of ligand-receptor interactions between nanoparticle and cell surfaces. Phys. Rev. Lett. 109, 1-5 (2012).

55. Curk, T., Dobnikar, J. \& Frenkel, D. Optimal multivalent targeting of membranes with many distinct receptors. Proc. Natl. Acad. Sci. U.S.A. 114, 7210-7215 (2017).

56. Sakai, T., Nishimura, S. I., Naito, T. \& Saito, M. Influenza A virus hemagglutinin and neuraminidase act as novel motile machinery. Sci. Rep. 7, 45043 (2017).

57. de Vries, E., Du, W., Guo, H. \& de Haan, C. A. M. Influenza A Virus HemagglutininNeuraminidase-Receptor Balance: Preserving Virus Motility. Trends Microbiol. 28, 57-67 (2020).

58. Plutnar, J. \& Pumera, M. Chemotactic Micro- and Nanodevices. Angew. Chem. Int. Ed. 58, 2190-2196 (2019).

59. Bartelt, S. M., Steinkühler, J., Dimova, R. \& Wegner, S. V. Light-Guided Motility of a Minimal Synthetic Cell. Nano Lett. 18, 7268-7274 (2018).

60. Ausländer, S., Ausländer, D. \& Fussenegger, M. Synthetic Biology-The Synthesis of Biology. Angew. Chem. Int. Ed. 56, 6396-6419 (2017).

61. Mesecar, A. D. \& Ratia, K. Viral destruction of cell surface receptors. Proc. Natl. Acad. Sci. U.S.A. 105, 8807-8808 (2008).

62. Tregubov, A. A., Nikitin, P. I. \& Nikitin, M. P. Advanced Smart Nanomaterials with Integrated Logic-Gating and Biocomputing: Dawn of Theranostic Nanorobots. Chem. Rev. 118, 1029410348 (2018). 


\section{Summary}

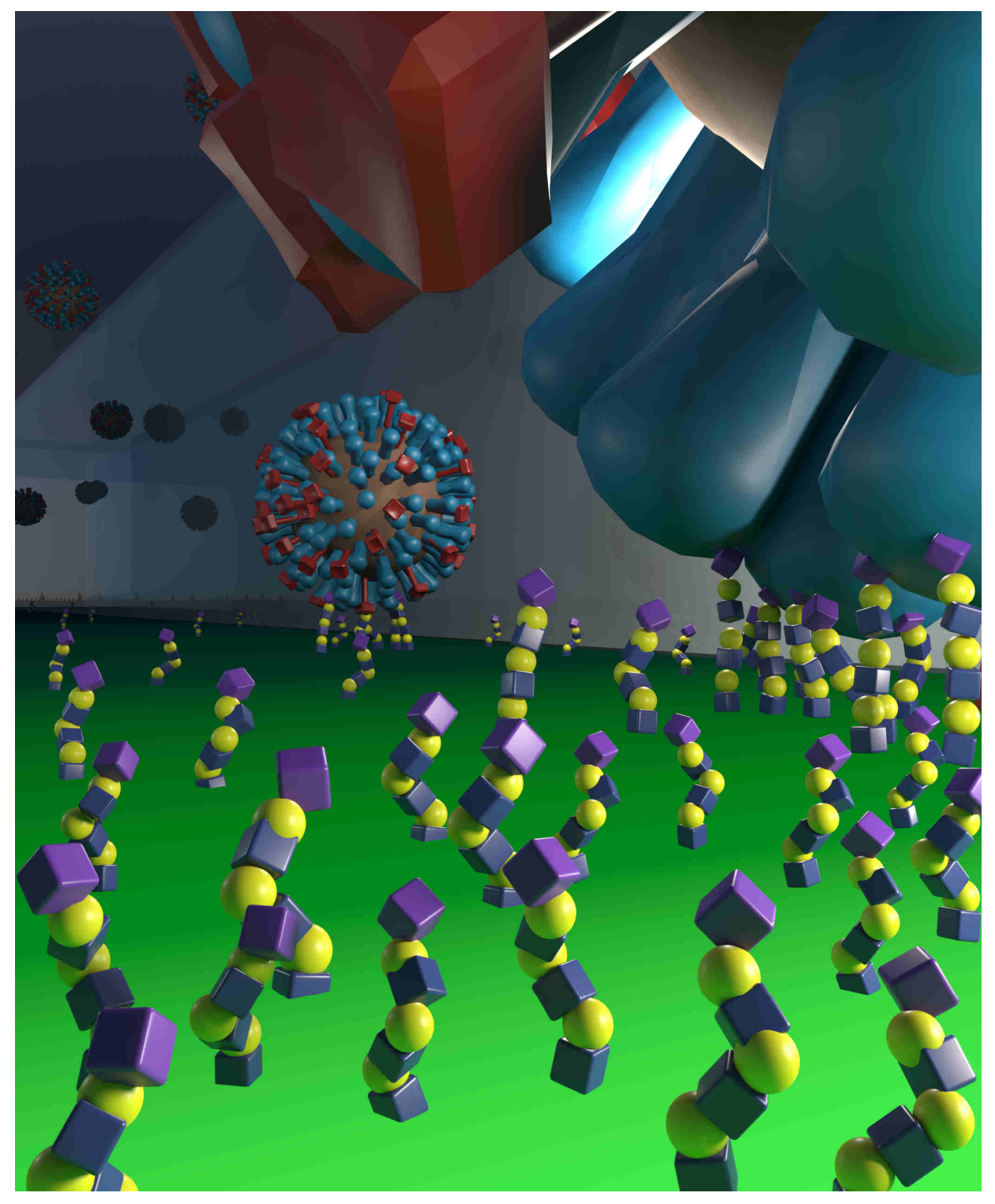




\section{Summary}

Influenza A viruses are responsible for seasonal epidemics and occasional pandemics (Chapter 1). These viruses originate in waterfowl but may adapt to humans after zoonotic infections. The surface of influenza viruses is decorated with two glycoproteins (Figure 1a), hemagglutinin (HA) and neuraminidase (NA), that form the characteristic "spikes" and are responsible for host cell recognition. The receptor specificity on HA and NA is considered a key factor in the adaptation of the virus to different hosts. HA binds specifically to sialic acidterminated glycans (Figure 1b). The HA of avian influenza binds preferentially to sialic acid that is linked to the penultimate galactose by an $\alpha 2,3$-linkage, whereas human influenza HA favors $\alpha 2,6$-linked sialic acid (Figure 1c). NA binds to the same receptors as HA and cleaves the bond between sialic acid and galactose (Figure 1b). Together, the virus uses its HA and NA to form multivalent interactions with the cell (Chapter 2). These multivalent interactions are more stable than the sum of their parts and have several emergent properties (Figure 1d): the virus binds superselectively to areas with higher receptor density (I), the virus can recruit mobile receptors towards the contact area (II), and the virus and surface can form a dynamic complex that alternates between different bound states (III). Several techniques to assess the multivalent binding and receptor specificity of influenza viruses have been reviewed. A representative and well-defined surface chemistry is critical when the binding of influenza viruses is studied on model systems.

In the initial stages of influenza infection, HA and NA work together in host cell recognition (Figure 1e). A functional balance exists between the binding and cleaving functions of the two glycoproteins that keeps the virus-receptor interactions dynamic. Additionally, the cleaving of receptors provides a driving force that allows the virus to efficiently cross the mucus, which is rich in decoy receptors (I). On the cell surface, viruses may find a suitable location for endocytosis by a combination of active motility (II) and lateral diffusion on mobile receptors (III). Endocytosis is induced by the recruitment of various membrane proteins that display sialic acid-terminated glycans (IV). 


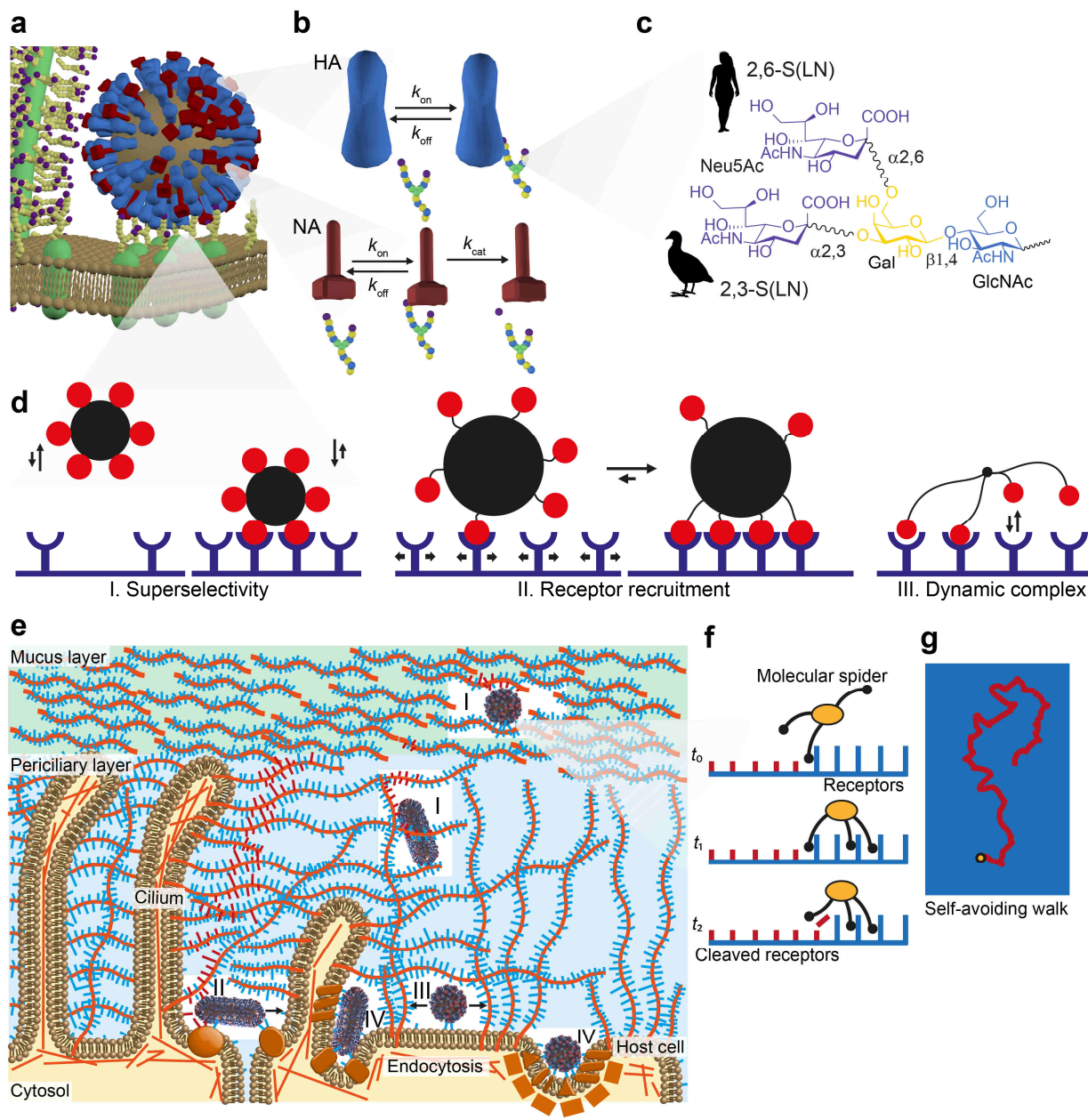

Figure 1| Interactions of influenza virus. a) Influenza virus in complex with receptors on a cell membrane. b) Interactions of HA and NA with sialoglycans. c) Structure of 2,3 and 2,6-linked sialoglycans. d) Emergent properties of multivalent interactions. e) Interactions of influenza virus with the mucus and host cell. f) Walking mechanism of a molecular spider. g) Path of a self-avoiding walk over a surface.

In this thesis, a method is presented (Figure 2), called "multivalent affinity profiling" to quantitatively assess the multivalent binding of influenza viruses on cell-mimicking surfaces (Chapters 3-6). Supported lipid bilayers (SLBs) are formed inside a microfluidic flow cell on a glass chip with interdigitated electrodes (I). The SLBs are formed from neutral lipids that are in the gel state 
at room temperature with a small percentage of charged biotinylated lipids. Stable biotin gradients are formed by applying a potential over the electrodes at elevated temperature and then cooling the chip to room temperature (II). The biotin gradient is functionalized with fluorescently labelled streptavidin and biotinylated glycans to form a receptor density gradient (III). Fluorescently labeled influenza viruses are passed through the flow cell and their binding as function of receptor density is imaged with fluorescence microscopy (IV). The multivalent virus-surface binding has a threshold receptor density above which the binding becomes efficient, which is the hallmark of the multivalent nature of the binding process $(\mathrm{V})$.

I
II

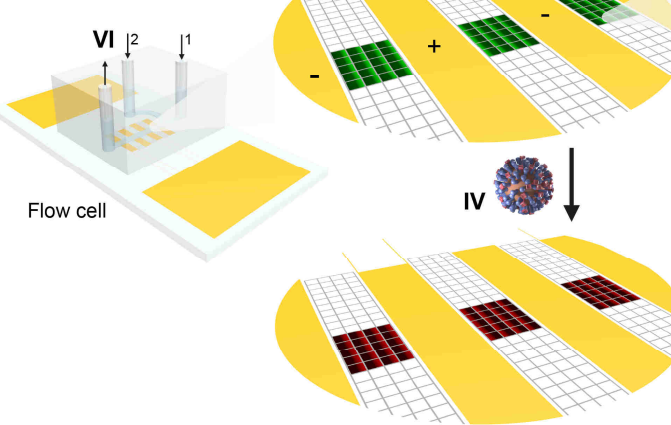

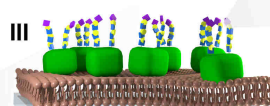

Glycans Streptavidin SLB

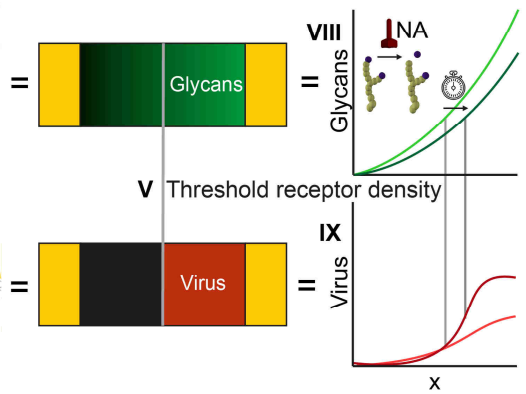

Figure 2| Method and applications of multivalent affinity profiling (MAP).

The flow cells were tested as a sensor device using a biotinylated fluorescent dye and streptavidin-coated fluorescent nanoparticles (Chapter $\mathbf{3}$ ). The binding of these fluorescent analytes in the sensing area was monitored over time and simulated in a finite element model. The fluorescent dye arrived late in the sensing area because it was depleted by nonspecifically bound SLB components in the tubing before reaching the flow cell. The nanoparticles were also depleted, but not delayed. A second inlet (VI) was added to the flow cell design to separate the nonspecifically bound SLB components from the analytes and avoid depletion in the tubing. 
The binding of influenza virus PR8 on receptor density gradients was studied using $\alpha 2,3$ and $\alpha 2,6$-linked glycans of different lengths as receptors (Chapter 4). The colocalization of virus and receptors was imaged using fluorescence microscopy to determine the threshold receptor density (V). A theoretical model is presented to calculate the avidity, the number of HA-receptor interactions, and their average strength from the threshold receptor density. PR8 had the same avidity for long $\alpha 2,3-$ and $\alpha 2,6$-linked glycans but the avidity was much lower for short $\alpha 2,6$-linked glycans and only slightly lower for short $\alpha 2,3$-linked glycans. The conformations in which streptavidin-bound glycans on the surface could bind to HA were compared using molecular dynamics simulations (VII). The differences in avidity originate from intrinsic structural and energetic differences in the interaction of $\alpha 2,3$ and $\alpha 2,6$-linked glycans with $\mathrm{HA}$, which are translated through receptor length and density into virus specificity and avidity.

Imaging virus binding on receptor density gradients allowed a direct visualization of the superselectivity of the virus (Chapter 5). The image processing to obtain binding profiles from fluorescence micrographs is explained, and the experimental conditions under which the threshold receptor density may be determined are evaluated. The threshold receptor density is stable over time and can be determined if the fluorescence from the virus is high enough and the local receptor density can be derived from the composition of the SLB. The threshold receptor density increases nonlinearly with flow rate due to the shear force exerted on the virus particles.

The influence of NA on the binding of influenza virus as a function of receptor density is studied by varying the concentration of NA inhibitor (Chapter 6 ). With active NA, the virus has a higher avidity due to the contribution of NA to the binding, but the receptor-cleaving activity of NA lowers the receptor density (VIII). The virus binding is decreased overall but more strongly at low receptor densities, leading to an enhanced selectivity towards high initial receptor densities (IX). At the level of individual viruses, both their binding and structure are highly heterogeneous.

The receptor-cleaving action of NA provides a driving force for influenza viruses to move through the mucus and over the surface of cells (Figure 1e, I and II), which resembles the motility of artificial receptor-cleaving particles called 
"molecular spiders" (Chapter 7). This type of molecular walkers also cleaves its receptors (Figure 1f), which leads to self-avoiding motion across a surface (Figure 1g). Rapid and efficient motility of a molecular spider is achieved when the binding and cleaving rates are balanced. The study of artificial molecular walkers could provide new insights about the motility of influenza viruses.

The insights in the multivalent binding of influenza viruses that are discussed in this thesis may help to predict the tropism and assess the zoonotic potential of emerging influenza strains, when combined with quantitative glycomics (Chapter 8). MAP may be used to assess the multivalent binding of other viruses as well, including SARS-CoV-2. Understanding the multivalent binding of viruses is a guiding motif in the development of broad-spectrum antivirals. Like viruses, targeted anticancer treatments can benefit from superselective binding. MAP can help in the design and development of superselective drug carriers. Lastly, the motility of influenza viruses challenges the view of viruses as simply containers for their genome and may inspire new biomedical nanodevices. 


\section{Samenvatting}

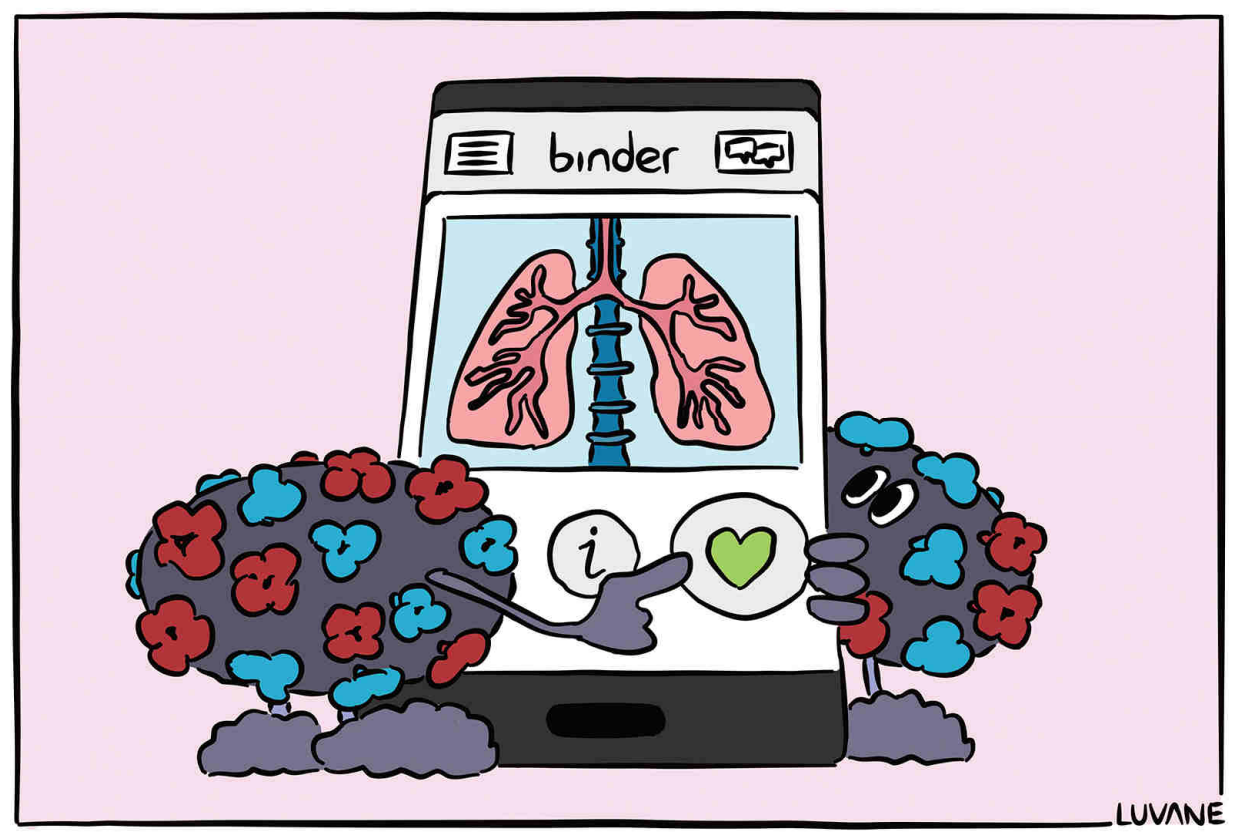




\section{Samenvatting}

Dit proefschrift beschrijft de interacties van een griepvirus met receptoren op een cel en presenteert een nieuwe methode om deze interacties kwantitatief te bestuderen.

Griepvirussen, waarvan het influenza A virus het meest voorkomt, veroorzaken elk jaar een epidemie en hebben door de jaren heen verschillende pandemieën veroorzaakt. In Hoofdstuk 1 wordt uitgelegd hoe griepvirussen zich aanpassen aan nieuwe soorten en wat de structuur van een griepvirus is. Een griepvirus kan bolvormig of langwerpig zijn en heeft een diameter van ongeveer $100 \mathrm{~nm}$. De buitenkant van het virus is bedekt met "spikes" die gevormd worden door de eiwitten hemagglutinine (HA) en neuraminidase (NA) (Figuur 1a). Deze spikes gebruikt het virus om gastheercellen te herkennen. HA kan een zwakke binding aangaan met receptoren die op deze cellen voorkomen (Figuur 1b). Deze receptoren zijn samengesteld uit verschillende suikers en eindigen in een siaalzuur, dat op twee manieren aan de voorafgaande suiker gekoppeld kan zijn (Figuur 1c). De HA van vogelgriep bindt bij voorkeur aan "rechte" 2,3receptoren, terwijl de HA van menselijke griep bij voorkeur "gebogen" 2,6receptoren bindt. NA is een enzym dat bindt aan dezelfde receptoren als HA maar de binding tussen het siaalzuur en de volgende suiker doorknipt (Figuur 1b).

In Hoofdstuk 2 wordt uitgelegd hoe HA en NA samen "multivalente" interacties aangaan met een cel (Figuur 1a). Deze multivalente interacties zijn stabieler dan de som van de individuele interacties en geven het virus drie eigenschappen die het niet zou hebben als het maar één binding tegelijk zou kunnen maken: Ten eerste is het virus "superselectief", hetgeen betekent dat het in één stap veel sterker kan binden op een oppervlak waar de dichtheid van receptoren hoog genoeg is om meerdere bindingen tegelijk te vormen (Figuur 1d). Ten tweede kan het virus mobiele receptoren rekruteren naar de plek waar het virus aan de cel bindt (Figuur 1e). Ten derde kan het virus een dynamisch complex vormen met een cel waarbij het gebonden blijft aan de cel, maar ondertussen voortdurend wisselt tussen verschillende receptoren (Figuur 1f). Er bestaan verschillende technieken waarmee de sterkte en specificiteit van de 
multivalente interacties van griepvirussen gemeten kunnen worden. Om aan de hand van zulke metingen griepvirussen beter te begrijpen is het vooral belangrijk dat bekend is hoe het oppervlak dat in die technieken gebruikt wordt eruitziet en dat het voldoende lijkt op die biologische omgeving die erdoor nagebootst wordt.

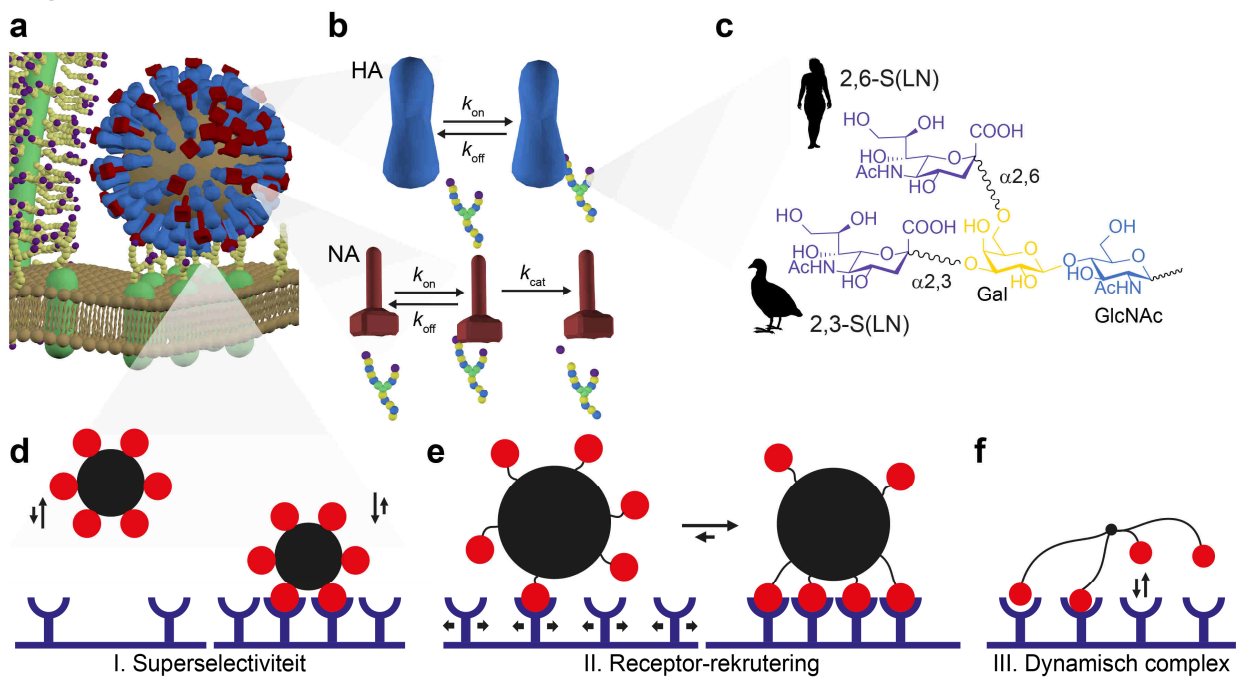

Figuur 1| Interacties van het griepvirus. a) Een griepvirus gebruikt zijn "spikes" om te binden aan meerdere receptoren tegelijk en vormt zo een multivalent complex. b) HA kan binden aan receptoren. NA kan binden aan receptoren en kan siaalzuur (paars) van een receptor afknippen zodat het griepvirus er niet meer aan kan binden. c) De receptoren van het griepvirus zijn complexe suikers die eindigen in drie monomeren: siaalzuur (paars), galactose (Gal, geel) en $\mathrm{N}$-acetylglucosamine (GlcNAc, blauw). Bij vogels komt de rechte 2,3-isomeer meer voor en bij mensen de gebogen 2,6-isomeer. d) Superselectiviteit: een virus bindt veel sterker als de receptordichtheid zo hoog is dat het meerdere bindingen tegelijk kan vormen. e) Receptor-rekrutering: als een virus aan mobiele receptoren bindt, wordt de receptordichtheid plaatselijk hoger. f) Dynamisch complex: als de individuele bindingen zwak zijn, maar de multivalente binding sterk, kan een virus aan een oppervlak gebonden blijven terwijl het steeds andere receptoren zijn waaraan het bindt.

Om een cel te infecteren, werken HA en NA samen. Er bestaat een balans in de bindfunctie en de knipfunctie van die twee eiwitten die ervoor zorgt dat de virus-receptor interacties dynamisch blijven. Daarnaast zorgt het knippen van receptoren voor een drijvende kracht die het virus efficiënt laat bewegen door de mucuslaag, waarin veel suikers zitten die de onderliggende cellen tegen 
infecties beschermen (Figuur 2a, I). Op een cel kunnen virussen bewegen naar een geschikte plaats om de cel binnen te dringen door een combinatie van actieve beweging als gevolg van het knippen van receptoren (II) en passieve diffusie door mee te liften op mobiele receptoren (III). Het virus dringt de cel binnen door verschillende membraaneiwitten te rekruteren waarop suikers met siaalzuur te vinden zijn (IV).

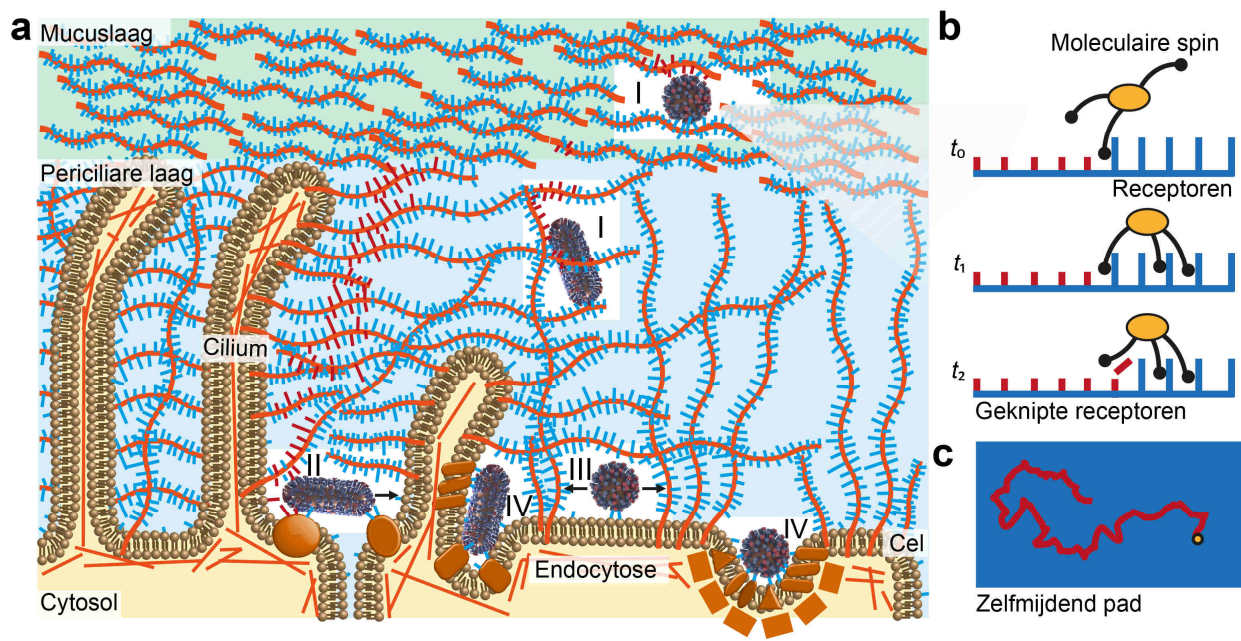

Figuur 2| Beweging van een griepvirus. a) Interacties van een griepvirus met de mucus en de gastheercel. b) Mechanisme waarmee een moleculaire spin loopt. c) Pad van een deeltje dat een zelfmijdende wandeling maakt op een oppervlak.

In de Hoofdstukken 3-6 presenteren we een techniek die we "multivalent affinity profiling" (MAP) noemen. Met deze techniek kunnen we de multivalente binding van griepvirussen kwantitatief bepalen op een oppervlak dat lijkt op een celmembraan. Hiertoe vormen we lipidenmembranen (supported lipid bilayers, SLBs) in een microkanaal op een glazen chip (Figuur 3a), tussen elektrodes die op de chip zijn aangebracht (Figuur 3b). Deze membranen bestaan voor het grootste deel uit ongeladen fosfolipiden die bij kamertemperatuur een gel vormen, maar bij hogere temperatuur vrij kunnen bewegen, met daarbij een klein percentage negatief geladen fosfolipiden met biotine aan de hydrofiele "kop". Bij $50{ }^{\circ} \mathrm{C}$ wordt een potentiaal aangebracht op de elektroden zodat de geladen fosfolipiden met biotine richting de positieve elektroden bewegen en zo gradiënten vormen. Door de chip af te koelen worden deze gradiënten stabiel gemaakt. Aan de biotinegradiënten binden we streptavidine, een eiwit met vier bindingsdomeinen voor biotine (Figuur 3c). Dit 
streptavidine is groen gekleurd met een fluorescent label. Op de twee vrije bindingsdomeinen aan de bovenkant van streptavidine worden receptoren met biotine gebonden zodat receptorgradiënten ontstaan (Figuur 3c). Dan voegen we griepvirussen met een rood fluorescent label toe die binden aan de receptoren (Figuur $3 b$ ). Het bindingsprofiel van de griepvirussen als functie van receptordichtheid wordt bepaald uit foto's gemaakt met een fluorescentiemicroscoop. De bindingssterkte van de griepvirussen neemt in één stap toe bij de drempelreceptordichtheid (Figuur 3d) wat kenmerkend is voor multivalente interacties.

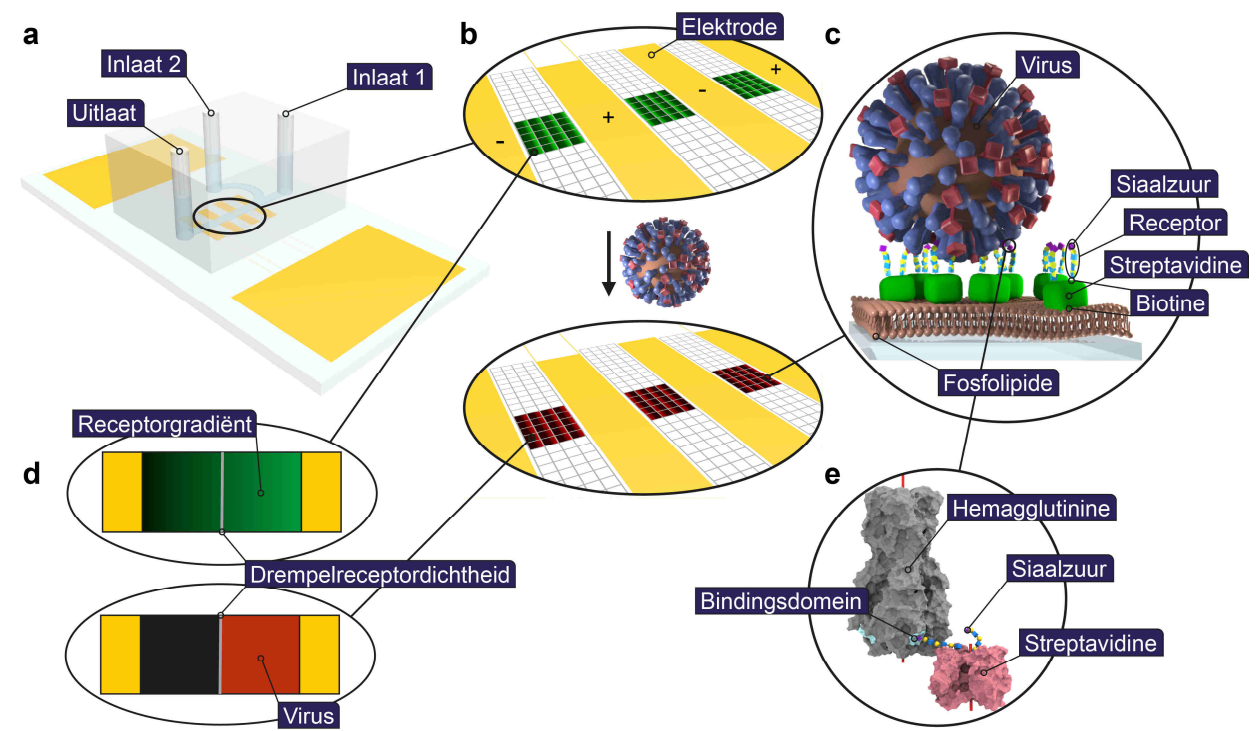

Figuur 3| De MAP-techniek. a) De microfluïdische chip waarin we gradiënten in lipidenmembranen maken met behulp van vier elektroden. b) De drie posities tussen de elektroden zijn onderverdeeld in vierkante cellen van $100 \mu \mathrm{m}$ waarin receptorgradiënten gevormd worden. De binding van griepvirussen op deze gradiënten wordt in beeld gebracht met behulp van een fluorescentiemicroscoop. c) Structuur van een lipidenmembraan met receptoren en gebonden virus. De componenten zijn niet op schaal. d) Het affiniteitsprofiel van het virus als functie van receptordichtheid maakt een "stap" bij de drempelreceptordichtheid die daardoor afgelezen kan worden. e) Met "molecular dynamics"-computersimulaties hebben we onderzocht welke oriëntaties van de verschillende receptoren in staat zijn om een binding met HA te vormen. Het bindingsdomein van HA is zo gepositioneerd dat vogel-type-receptoren (zoals hier afgebeeld) van opzij erin passen, terwijl receptoren van het menselijk type dicht tegen HA aan liggen als ze gebonden zijn. 
In Hoofdstuk 3 testen we de microfluïdische chip als sensor met behulp van een fluorescente kleurstof met biotine en fluorescente nanodeeltjes met streptavidine. We hebben de binding van deze analyten in het sensorgedeelte gemeten in de tijd en gesimuleerd met een "finite element"-model. De kleurstof kwam vertraagd aan in het sensorgebied doordat het bond aan componenten van het lipidenmembraan die aan de wand van de inlaatslang geadsorbeerd waren. Van de nanodeeltjes bleef wel een deel achter in de inlaatslang, maar dat zorgde er niet voor dat ze vertraagd aankwamen. Door een tweede inlaatslang toe te voegen aan het ontwerp van de microfluïdische chip konden we de te detecteren stoffen gescheiden houden van de lipiden die in de inlaatslang achterbleven en konden we voorkomen dat deze stoffen achterbleven.

In Hoofdstuk 4 wordt de binding van het griepvirus "PR8" gemeten op gradiënten van receptoren van het 2,3- en 2,6-type met verschillende lengtes om beter te begrijpen waar de specificiteit van griepvirussen voor bepaalde receptoren vandaan komt. Met een fluorescentiemicroscoop hebben we de dichtheden van receptoren en gebonden virus in beeld gebracht om daaruit de drempelreceptordichtheid te bepalen. Met een theoretisch model beschrijven we hoe uit de drempelreceptordichtheid niet alleen de totale sterkte van de multivalente binding bepaald kan worden, maar ook het aantal interacties dat bijdraagt aan de binding en de sterkte van de individuele interacties. Het griepvirus PR8 bond even sterk aan lange receptoren van het vogeltype als aan het menselijke type en bond veel zwakker aan korte menselijke receptoren, terwijl de binding aan korte vogeltype receptoren maar iets zwakker was. Met molecular dynamics computersimulaties hebben we vergeleken welke vormen en oriëntaties van de receptoren in staat waren om aan de HA van het virus te binden (Figuur 3e). Daaruit bleek dat het verschil in de sterkte van de multivalente binding ontstaat uit het verschil in oriëntatie van de receptoren van het vogeltype en het menselijk type in het bindingsdomein van HA met de verschillende bindingsenergieën die daarbij horen. Deze verschillen tussen het vogeltype en menselijk type leiden in combinatie met de lengte van de receptoren en hun dichtheid op het oppervlak tot een verschillende specificiteit en bindingssterkte van het virus.

Hoofdstuk 5 laat zien dat het in beeld brengen van de dichtheid van receptoren en gebonden virussen een rechtstreekse visualisatie van superselectiviteit 
(Figuur 1d) mogelijk maakt. Het proces om bindingsprofielen van het virus te halen uit fluorescentiefoto's van het virus en de receptorgradiënten wordt uitgelegd en de experimentele voorwaarden om de drempelreceptordichtheid uit deze foto's te halen worden besproken. De drempelreceptordichtheid is stabiel in de tijd en kan vastgesteld worden als de fluorescentie van de gebonden virussen hoog genoeg is én de plaatselijke receptordichtheid bepaald kan worden op basis van de samenstelling van het lipidenmembraan. De drempelreceptordichtheid neemt niet-lineair toe als we de stroomsnelheid door het microkanaal verhogen vanwege de afschuifkracht van het water op de virusdeeltjes.

In Hoofdstuk 6 wordt de rol van NA onderzocht in de binding van griepvirussen. Hiertoe hebben we het griepvirus PR8 op een receptorgradiënt te laten binden bij verschillende concentraties NA-remmer. Met actieve NA bindt het virus sterker, maar het knippen van receptoren verlaagt tegelijk ook de receptordichtheid zodat het virus uiteindelijk minder interacties kan vormen. Actieve NA zorgt ervoor dat over het geheel minder virus bindt. Dit effect is het sterkst bij lage receptordichtheden en versterkt daarmee de selectiviteit die het virus heeft voor hogere receptordichtheden. Op het niveau van de individuele virussen lopen zowel hun binding als hun structuur ver uiteen.

Hoofdstuk 7 laat zien dat het knippen van receptoren door NA leidt tot een drijvende kracht waarmee griepvirussen efficiënt kunnen bewegen door de mucuslaag en over het oppervlak van cellen (Figuur 2a, I en II) op een manier die veel lijkt op de beweging van kunstmatige deeltjes die ook hun receptoren knippen, de zogenaamde "moleculaire spinnen" (Figuur 2b). Het knippen van receptoren door deze deeltjes leidt tot een zelfmijdende wandeling over een oppervlak (Figuur 2c), wat een efficiëntere beweging is dan willekeurige diffusie. Als het binden en knippen van een moleculaire spin in balans zijn, kan ze snel en efficiënt over een oppervlak bewegen. Het bestuderen van kunstmatige wandelende deeltjes kan helpen om beter te begrijpen waardoor het wandelen van griepvirussen bepaald wordt.

Hoofdstuk 8 beschrijft hoe de inzichten uit dit proefschrift gebruikt kunnen worden voor toekomstig onderzoek. Als de dichtheid van verschillende receptoren in de luchtwegen beter in kaart gebracht wordt, kan de MAPtechniek gebruikt worden om te voorspellen op welke cellen nieuwe 
griepvirussen binden. Daarmee kunnen ook voorspellingen gedaan worden over de besmettelijkheid, de ernst van de ziekte en het risico op overdracht van dier naar mens van nieuwe griepvirussen. De MAP-techniek kan ook gebruikt worden om de multivalente binding van andere soorten virussen te meten, bijvoorbeeld SARS-CoV-2 dat COVID-19 veroorzaakt. Nieuwe inzichten in de multivalente binding van griepvirussen kunnen worden gebruikt voor de ontwikkeling van breed inzetbare antivirale middelen. Net als virussen kunnen ook gerichte medicijnen tegen kanker voordeel hebben van superselectieve binding. De MAP-techniek kan daarom helpen in het ontwerpen en ontwikkelen van superselectieve formuleringen voor medicijnen. Tenslotte impliceert het wandelen van griepvirussen dat virussen een veel actievere rol hebben dan vaak gedacht en kan het een bron van inspiratie vormen voor nieuwe biomedische nanomachines. 


\section{Acknowledgements}

One name on the cover does not mean I did the work in this thesis alone. I had the pleasure to work in a collaborative, challenging, stimulating, but most of all supportive environment. There was always an ear for cheers and sighs, and a hand to help. Before all, I thank the LORD my God who brought all these amazing people on my path.

I thank Jurriaan Huskens, my promotor, for his exceptional readiness to guide and help. Het is echt bijzonder hoe jij je ingespannen hebt om er te zijn. In vier jaar tijd kan ik me niet één keer herinneren dat je ergens te laat op reageerde: als ik binnen een uur reactie nodig had, dan kreeg ik dat binnen een halfuur; als iets een paar maanden mocht liggen, dan liet je het ook niet meer dan een paar maanden liggen. Je nam ook de tijd om samen koffie te drinken of een biertje, of om samen een model te bedenken en door te rekenen. Zowel in het formele als informele ben je een geweldige mentor geweest. Al tijdens mijn master stimuleerde je om verder te denken en door te vragen. Jouw manier van vragen stellen is beslist een voorbeeld voor me geweest. Je hebt me in het schrijfproces van begin tot eind begeleid. Jouw schrijfcursus (first logic, then language) heeft daarin ook veel geholpen. Je positieve houding nodigde ook echt uit om af en toe iets voor de groep te organiseren.

I thank Erhard van der Vries, my co-promotor, for showing me the biological context. Door de discussies met jou, ben ik iets gaan begrijpen van de complexiteit waarin alles zich afspeelt. Behalve de experimenten, materialen en contacten die je bijdroeg, nam je ook de tijd om samen uit te zoeken wat de data betekende, daar samen over door te denken en een koppeling te maken naar de bredere context.

Thanks, Rainer Haag for hosting my favorite conferences. I very much appreciated all the helpful discussions in Berlin, and I believe your efforts to involve students played a part in creating this atmosphere. It also helped that you provided us with enough chocolate for the uncertain way home. Thanks, Menno Prins for your thorough evaluation of my concept thesis and many valuable comments. Thanks, Armagan Kocer for your interest and comments. 
Thanks, Pascal Jonkheijm for introducing me to organic and biomimetic chemistry and for bringing lipid bilayers into our group. I am glad you all agreed to join my committee.

I thank my paranymphs, Erik Hamming and Ruben Kolkman. Erik, we hebben een bijzondere samenwerking gemaakt waarbij we de helft van de tijd konden kletsen en toch meer gedaan krijgen dan alleen. Je bent een vriend geweest bij wie ik alles kwijt kon wat me bezighield, een maatje onderweg en een collega die precies goed was in wat ik moeilijk vond. Je hebt je ook bijzonder ingezet voor de groep en nam mij daarin mee. Ruben, je viel voor me in en nam de masterclass nanotechnologie van me over en we hebben samen Adam begeleid. Bedankt voor de fijne samenwerking!

I thank our collaborators: Nick Tito for his help with superselectivity theory. Malte Tieke for the production and labelling of influenza viruses, and for the Octet measurements. Geert-Jan Boons, Zeshi Li, and Gaël Vos for the synthesis of the glycans. Oliver Grant for the molecular dynamics simulations and Robert Woods for his help in writing our paper. Robert de Vries for his glycan array measurements, help with our article, and help with the microscale thermophoresis. Bart-Jan Ravoo and Wilke de Vries for the work on polymer shelled vesicles and giant vesicles with Yao. Kislon Voitchovsky and Miro Cafolla for hosting me in Durham for imaging viruses on lipid membranes with AFM, which sadly did not work, but still was fun. Kai Ludwig for the cryo-TEM imaging. Stephan Block for hosting Erik and me in his pristine lab in Berlin and risking his non-immersion lens with oil to track viruses for us. And all of you for the helpful discussions.

I thank our technical staff: Marcel, Regine, Richard, and Bianca, and our secretaries: Izabel and Nicole. Jullie hebben geholpen bij alles wat er te regelen viel van administratie tot zuurkast. Jullie bewaren alle mooie tradities in de groep en zorgden voor gezelligheid in de pauzes. Als iemand een leuk idee had, maakten jullie het mogelijk.

I thank Clemens Padberg for his help with the fluorescence microscope and Rico Keim for his help with the TEM. I also thank the support staff of the (former) Bionanolab, in particular Ine and Irene, for their help with the confocal microscope and other instruments. 
Yao Lu, thank you for the nice collaboration! I am glad you came back to continue working with us. Thank you, Daniele, Sara, and Cande for the QCM measurements, the virus discussions and all the fun times. Thanks, Wouter Vijselaar and Edo de Kruiff for making my chips in the cleanroom. Thanks, Mark Verheijden for teaching me how to work with vesicles, lipid bilayers, and flow cells, and how to make bad puns. Thanks, Jasper van Weerd for helping me with the flow cells. I thank Wim Verboom for helping me prepare for my attempts at synthesis.

I thank my students: Jelco Albertsma, Jolisa Rodriguez Cobos, Tren Fiers, Willeke Klappe, and Adam Walczak for all their work. Thanks Mike, for helping Adam in the Nanolab.

I thank Nicole Zeijen for being a friend through our PhDs, for helping me manage the lipids work, helping me figure out how to use COMSOL, and for teaching me what I needed to know about statistics. When I found it difficult to apply statistical tests, Bea helped me. Thank you, Albert, Yao, Ruben, Melissa, and Wouter for proofreading.

I thank Willem Vos for his insights in data processing and Annemarie Huijser for her insights in fluorescence quenching. I thank Erik de Vries, Xander de Haan, Hongbo Guo, and Albert Osterhaus for their insights about influenza viruses.

I thank my (former) colleagues, many of whom I count as friends, for making my work fun and my breaks work. Thank you all, for the insightful questions and discussions during the colloquia, subgroup meetings, and coffee breaks. Thanks for all the good times we shared at work, at conferences, and outside of work. Thank you, Jurriaan, Jeroen, Pascal, Wim, Jos, Nathalie, and Tibor, for your roles both as staff and personally in stimulating this culture in our group. Christian, Saskia, and Albert, you joined only recently and in a time of social distancing, but you fit in perfectly and I appreciate your efforts for the group.

I thank Robin, Luca, Erik, and Melissa for our pathfinder games, and with Elvera and Nicole for your companionship in work and leisure. Naomi, Richard, Jos, Nicole, and Erik for organizing the workweek with me. Mark de Ruiter, who introduced me to microbiology. Wouter Vijselaar, for introducing me to the masterclass nanotechnology, for broadening my scope, and for your own 
efforts to understand the work of other people. Leendert Noordam, my buddy through our masters, for starting it all when you heard Jurriaan had a vacancy, and for sharing your protocols for giant vesicles. Dodo, for sharing your experience and for your efforts to improve our lipids work. Aijie, Aina, Alberto, Alexander, Almu, Amber, Anamarija, Aref, Aukie, Axel, Chris, Christa, Dhanya, Diana, Dipak, Emanuela, Emma, Erick, Esli, Fangyuan, Federico, Fleur, Francisca, Fuad, Gaurav, Gigi, Gülistan, Hasnaa, He, Huub, Ines, Jacopo, Janita, Janneke, Jan-Willem, Jeltsje, Jéré, Jonathan, Joris, Julien, Kevin, Leonie, Lukacs, Luke, Luilin, Maaike, Maike, Manee, Mark, Maria, Matthijs, Melissa, Milou, Min, Muhabbat, Naomi, Nicola, Nicolas, Nienke, Noemi, Peng, Pia, Pramod, Qin, Quim, Rachèl, Remi, Rens, Rianne, Rindia, Rik, Roberto, Samer, Sander, Sandra, Sandro, Saskia, Shuqin, Sisi, Siyu, Stan, Supaporn, Taghi, Thijs, Timo, Tom, Twan, Verónica, Victoria, Virginia, Wouter, Yunita, thank you all!

Als laatste wil ik Bea, mijn familie, vrienden en gemeente bedanken voor alle steun en belangstelling.

Nico Overeem

Deventer, December 2020 


\section{About the author}

Nico Johan Overeem was born in Deventer on 2 March 1993. He studied chemical engineering at the University of Twente, where he obtained his BSC degree in 2014 and his MSc degree in 2016. The research for his BSc thesis entitled "Construction of a poly(ferrocenylsilane) - glucose oxidase thin film electrode" was performed under the supervision of Xueling Feng and Dr. Mark A. Hempenius in the group Material science and Technology of Polymers of Prof. Dr. G. Julius Vancso. He followed an internship at the Technion - Israel Institute of Technology in Haifa, Israel under the supervision of Adi Azran in the group Polymeric biomaterials: Structure - function relations of Prof. Dr. Havazelet Bianco-Peled on which he wrote a report entitled "Encapsulation of 5fluorouracil in biodegradable polymersomes". The research for his MSc thesis entitled "Encapsulation of enzyme cascades in a viral capsid" was performed under the supervision of Mark V. de Ruiter in the group Biomolecular Nanotechnology of Prof. Dr. Jeroen J. L. M. Cornelissen.

Since October 2016 he has worked as a PhD student in the group Molecular Nanofabrication under the supervision of Prof. Dr. Ir. Jurriaan Huskens in a collaborative project with Dr. Erhard van der Vries. His research was funded from the project "Influenza Virus Affinity Profiles on Quantitative Nano-chips" (FLAP-chips, Volkswagen Foundation), which aimed at characterizing and quantifying complex influenza virus-host cell interactions using biologically relevant dynamic, multivalent and superselective platforms, and from the project "Multivalent interactions at the sweet spot" (TOP 715.015.001, NWO), which aimed at developing an experimental toolbox in order to further our understanding of weak multivalent interactions in artificial and biological systems. 


\section{Publications}

5. Overeem, N. J., Hamming, P. H., Grant, O. C., Di lorio, D., Tieke, M., Bertolino, M. C., Li, Z., Vos, G., De Vries, R. P., Woods, R. J., Tito, N. B., Boons, G. J. P. H., Van der Vries, E., Huskens, J., Hierarchical Multivalent Effects Control Influenza Host Specificity, ACS Central Science, 2020, 6, 2311-2318

4. Overeem, N. J., Hamming, P. H., Huskens, J., Time-dependent Binding of Molecules and Nanoparticles at Receptor-Modified Supported Lipid Bilayer Gradients in a Microfluidic Device, ChemistrySelect, 2020, 5(31), 9799-9805

3. Hamming, P. H. ${ }^{\dagger}$, Overeem, N. J. ${ }^{\dagger}$, Huskens, J. Influenza as a molecular walker, Chemical Science, 2020, 11(1), 27-36

2. Lu, Y. ${ }^{\dagger}$, De Vries, W. C. ${ }^{\dagger}$, Overeem, N. J. ${ }^{\dagger}$, Duan, X., Zhang, H., Zhang, H., Pang, W. Ravoo, B. J., Huskens, J., Controlled and Tunable Loading and Release of Vesicles by Using Gigahertz Acoustics, Angewandte Chemie - International Edition, 2019, 58(1), 159-163

1. De Ruiter, M. V., Overeem, N. J., Singhai, G., Cornelissen, J. J. L. M., Induced Förster Resonance Energy Transfer by Encapsulation of DNA-Scaffold Based Probes inside a Plant Virus Based Protein Cage, Journal of Physics Condensed Matter, 2018, 30(18), 184002

† Equal authorship 


\section{Manuscripts}

6. Overeem, N. J., Van der Vries, E., Huskens, J., A Dynamic, Supramolecular View on the Multivalent Interaction Between Influenza Virus and Host Cell, Small - accepted

7. Overeem, N. J., Hamming, P. H., Tieke, M., Van der Vries, E., Huskens, J., Multivalent affinity profiling: direct visualization of the superselective binding of influenza viruses - submitted

8. Overeem, N. J., Hamming, P. H., Block, S., Ludwig, K. Fiers, T. J., Tieke, M., Kruiff, E. A. G., Van der Vries, E., Huskens, J., The role of neuraminidase in the superselective binding of the influenza virus - in preparation 